Thomas Schaefer

Adaption an Nestprädation bei der Mönchsgrasmücke

Göttingen 2002 



\title{
Adaption an Nestprädation bei der Mönchsgrasmücke
}

\author{
Dissertation zur Erlangung des Doktorgrades der \\ Mathematisch-Naturwissenschaftlichen Fakultäten der \\ Georg-August-Universität zu Göttingen
}

Angefertigt an der Max-Planck-Forschungsstelle für Ornithologie Vogelwarte Radolfzell

vorgelegt von:

Thomas Schaefer aus Krefeld

Göttingen 2002 
D7

Betreuer der Arbeit: Prof. Dr. P. Berthold

Referent: Prof. Dr. M. Schaefer

Korreferent: Prof. Dr. E. Schürmann

Tag der mündlichen Prüfung: 25.04.2002 


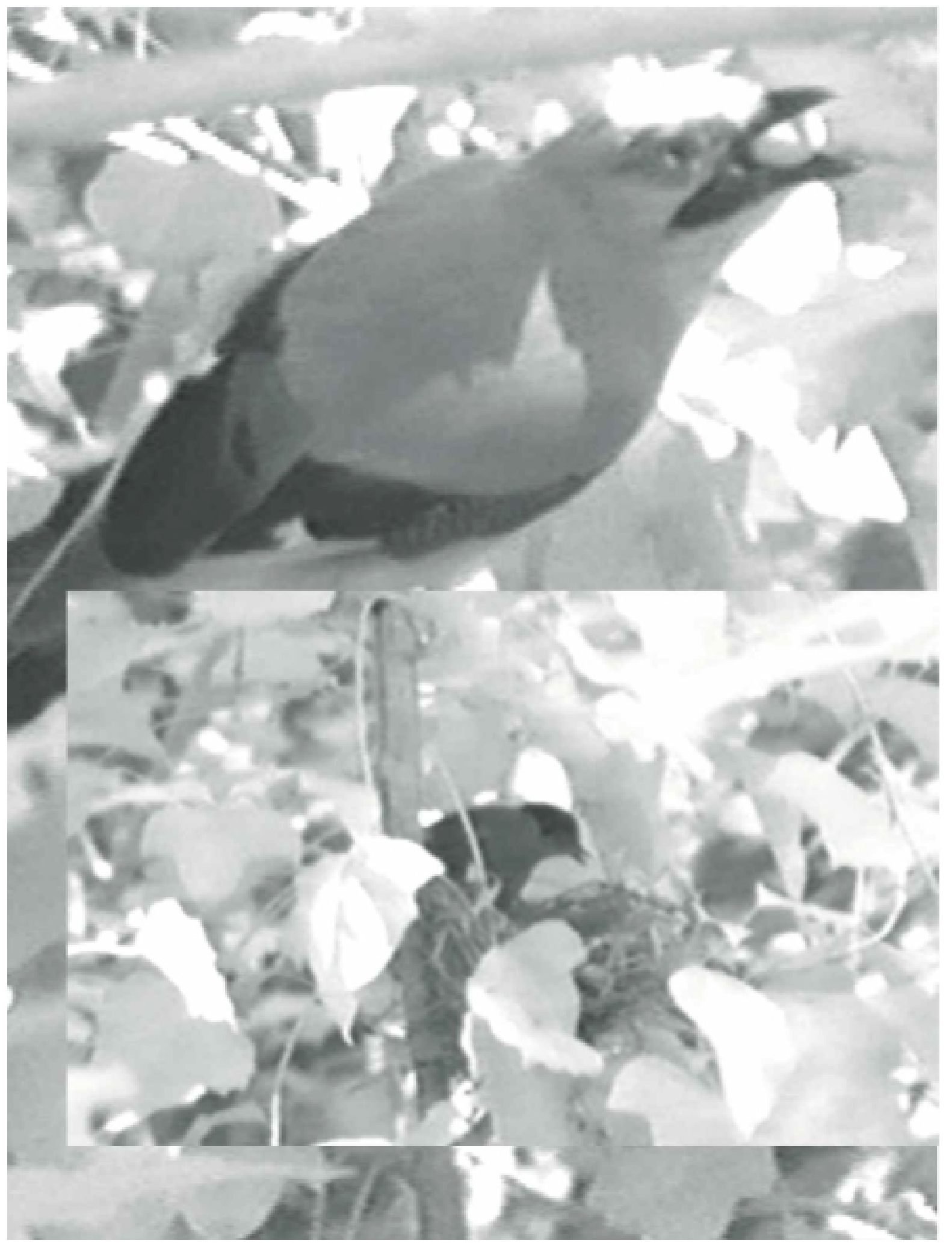

„Der Eichelhäher hat im Astquirl einer Fichte sein Nest gebaut - die Jungen sind vorm Ausfliegen [...] Die brütenden Kleinvögel im weiten Umkreise haben schwer zu leiden. Der Laubvogel [...] wird verjagt und die Eichelhäher morden und rauben die Nestlinge [...] der Grasmücke geht es nicht besser." 



\section{Inhaltsverzeichnis}

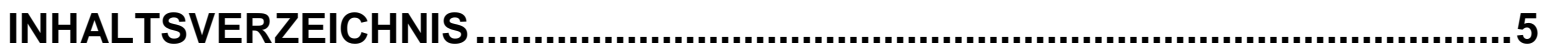

1 EINLEITUNG

1.1 Nestprädation als Selektionsfaktor bei Singvögeln.................................. 9

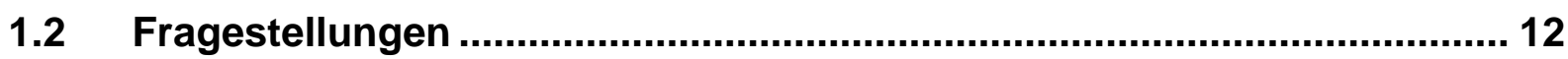

2 DIE MÖNCHSGRASMÜCKE ALS FORSCHUNGSGEGENSTAND .............15

3 UNTERSUCHUNGSGEBIETE UND METHODEN ......................................17

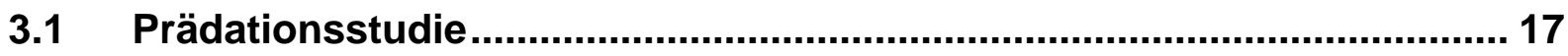

3.1.1 Untersuchungsflächen .............................................................. 17

3.1.1.1 Südwestdeutschland .......................................................... 17

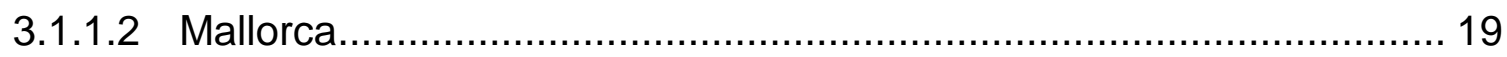

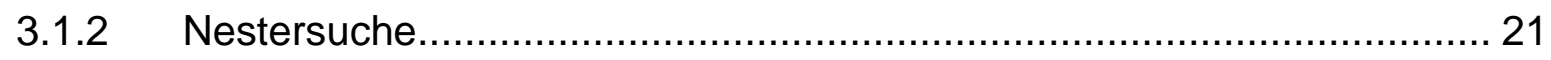

3.1.3 Videoüberwachung von Nestern der Mönchsgrasmücke....................... 21

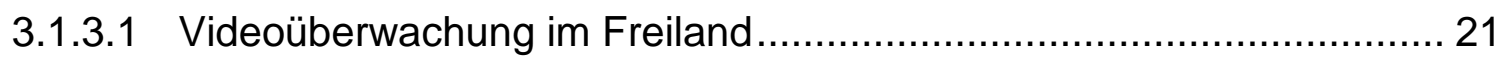

3.1.3.2 Aufstellen der Kameras .............................................................. 23

3.1.3.3 Erfahrungen beim Betrieb ………………............................... 24

3.1.3.4 Nach einem Prädationsereignis .................................................. 26

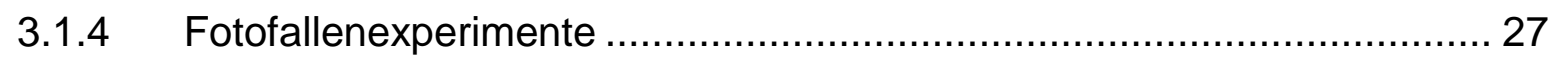

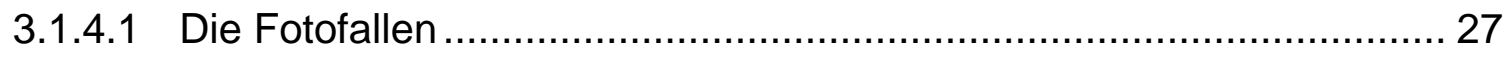

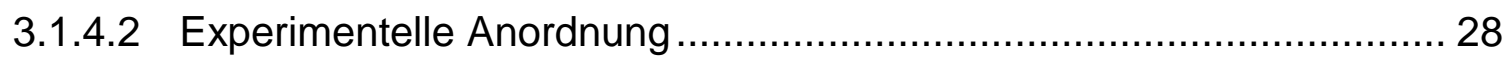

3.1.5 Erfassung von Neststandortparametern .......................................... 29

3.1.6 Kunstnestexperimente auf Mallorca und in Südwestdeutschland ........... 30 
3.1.7 Siedlungsdichte der Mönchsgrasmücke auf Mallorca

3.2 Daten aus Nestfunden an der Vogelwarte 1981-2000. 31

3.2.1 Untersuchungsflächen. 31

3.2.2 Nestersuche und Aufnahme brutbiologischer Parameter. 32

3.2.3 Untersuchungen zum Einfluss der Landschaft auf die Prädationsrate ....36

3.3 Auswertung von Brutdaten aus Volieren der Vogelwarte. 36

4 ERGEBNISSE 39

4.1 Prädationsmuster und Prädatoren an Nestern der Mönchsgrasmücke .. 40

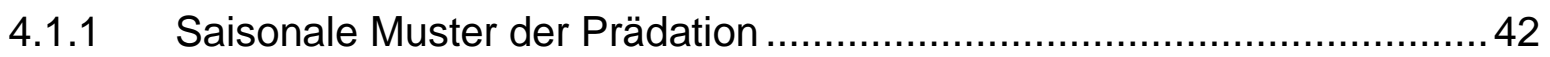

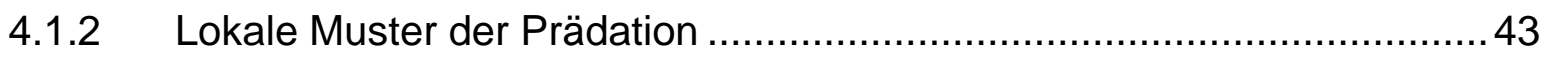

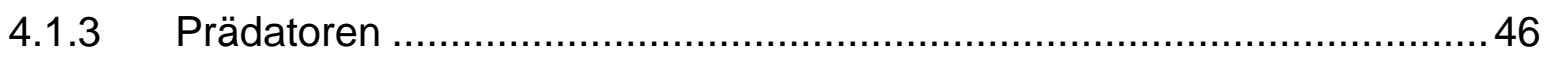

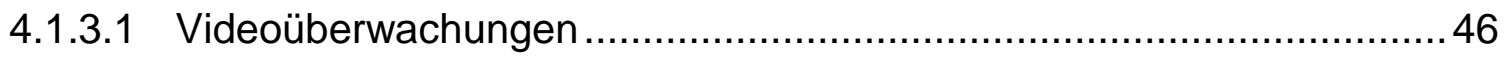

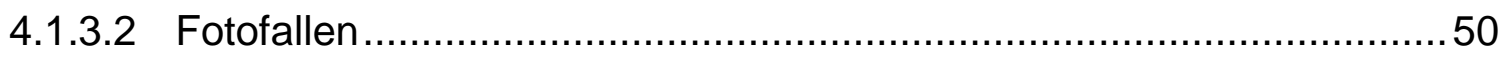

4.2 Reduktion der Prädationsrate .............................................................54

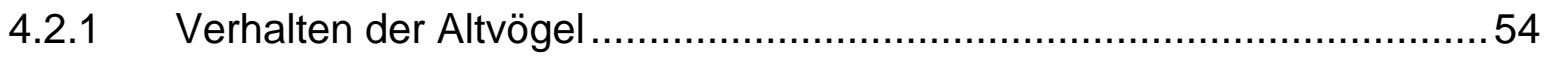

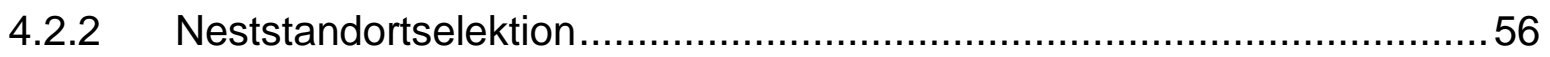

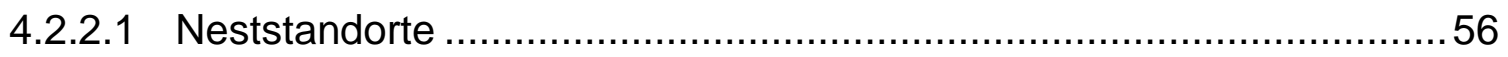

4.2.2.2 Prädation und Neststandorte ................................................... 59

4.2.2.3 Prädatoren und Neststandorte .................................................. 62

4.2.2.4 Wahl der Neststandorte und Prädation auf Mallorca .........................63

4.3 Reduktion des Prädationseffektes..........................................................66

4.3.1 Kompensation von Gelegeverlusten .............................................66

4.3.2 Brutbiologie, Gelegegröße und Überlebenswahrscheinlichkeit ..............67 
5.1 Prädationsmuster und Prädatoren an Nestern der Mönchsgrasmücke.. 73

5.1.1 Lokale Unterschiede der Prädationsrate........................................... 75

5.1.2 Prädatoren und ihr Verhalten ......................................................... 79

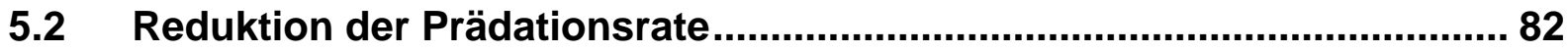

5.2.1 Heimliches Verhalten, Verteidigung und Reduktion der Gelegegröße.... 82

5.2.2 Neststandortselektion bei der Mönchsgrasmücke .............................. 85

5.3 Reduktion des Prädationseffektes ….................................................... 92

5.3.1 Kompensation von Brutverlusten ………….................................... 92

5.3.2 Brutbiologie, Gelegegröße und Überlebenswahrscheinlichkeit.............. 94

5.4 Ist die Mönchsgrasmücke durch den Eichelhäher bedroht? ................. 103

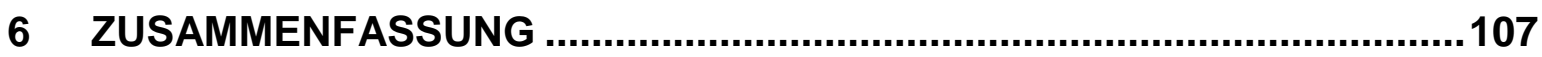

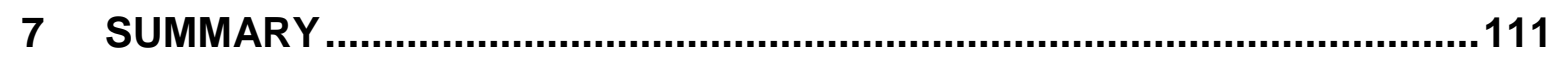

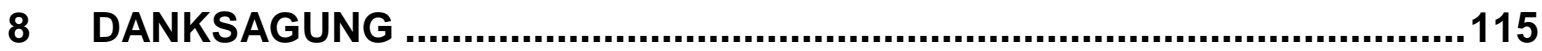

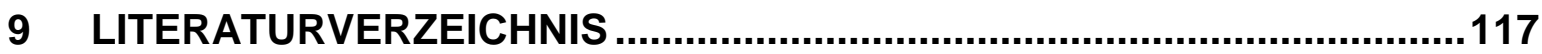

ANHANG I: TABELLENVERZEICHNIS.......................................................133

ANHANG II: ABBILDUNGSVERZEICHNIS ...............................................135 



\section{Einleitung}

\subsection{Nestprädation als Selektionsfaktor bei Singvögeln}

Nestprädation gilt als Hauptursache von Gelegeverlusten bei Singvögeln, weil durchschnittlich 80 \% der Brutverluste auf Prädation zurückgehen (RICKLEFS 1969, BEST \& StAUFFER 1980, MARTIN 1992). Im Mittel beträgt die Prädationsrate 50 \% (NICE 1957), in Einzelfällen kann dieser Wert bis auf über 90 \% steigen (MøLLER 1988, MARTIN \& ROPER 1988, SuAREZ ET AL. 1993, HANSKI \& LAURILA 1993). Wertet man das Ausmaß der Brutverluste nicht nach Erfolg/Misserfolg eines Nestes, sondern berechnet die Überlebenswahrscheinlichkeit (MAYFIELD 1961), scheinen Werte zwischen 0,25 und 0,35 die Regel zu sein (Hoover et Al. 1995, Sockman 1997, Davison \& Bollinger 2000). Minimalwerte liegen bei 0,07 (MARTIN \& ROPER 1988) und 0,12 (BARKOW ET AL. 2001), d.h. nur jedes zehnte Nest ist erfolgreich. Stochastisch gesehen benötigen Singvögel demnach zwischen zwei und zehn Versuche für eine erfolgreiche Brut. MARTIN (1988a) lenkte als einer der ersten den Blick auf Prädation als einen der wichtigsten Selektionsfaktoren bei Singvögeln. Noch bevor sich Fragen nach Habitatqualität und der daraus resultierenden Fitness von Jungvögeln sowie differenzierter Rekrutierungsrate im Folgejahr stellen, muss ein Nest erfolgreich gewesen sein. Ausgehend von der Bedeutung des Reproduktionserfolges für die individuelle Fitness (FUTUYMA 1990) sollte die natürliche Selektion Vögel fördern, Lebenszyklusmerkmale (,life history traits“, FUTUYMA 1990) auszubilden, die den negativen Effekt der Prädation reduzieren (MARTIN 1993A, MARTIN 1995).

Aus der Literatur können zwei Adaptionsmöglichkeiten von Singvögeln an Nestprädation abgeleitet werden (CRESSWELL 1997a): Reduktion der Prädationsrate und Reduktion des Prädationseffektes (Abb. 1). Die Prädationsrate kann einerseits durch eine Selektion heimlichen Verhaltens der Altvögel reduziert

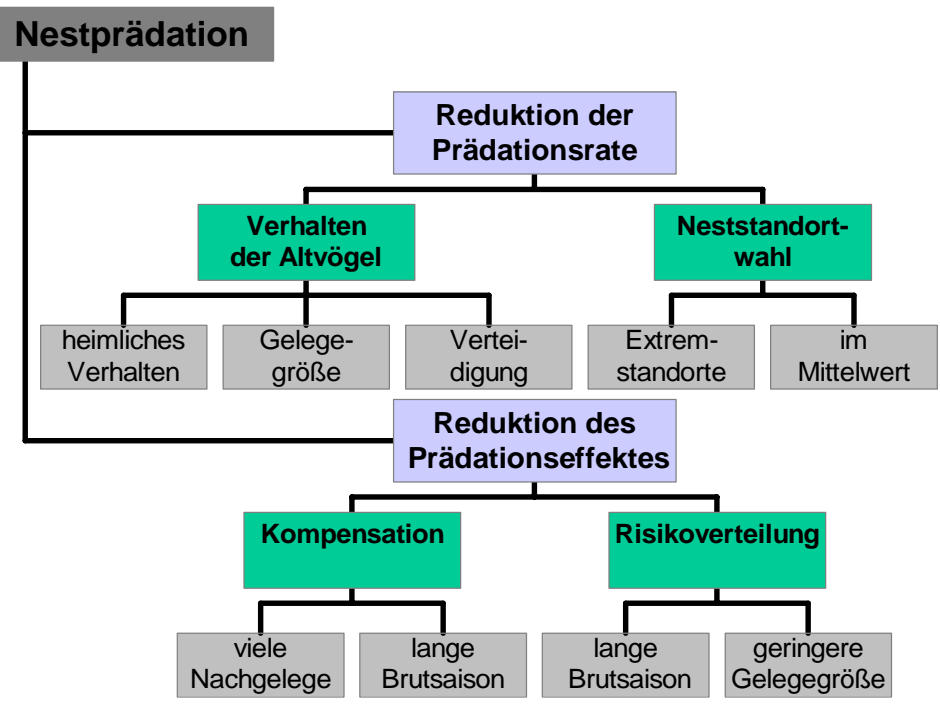

Abb. 1: Mögliche Adaptionen an Nestprädation. 
werden, die vermeiden, dass ihr Weg zum Nest verfolgt werden kann. In diesem Zusammenhang wird auch eine Reduktion der Gelegegröße diskutiert, weil durch eine geringere Fütterrate der Jungen die Aktivität am Nest weiter vermindert wird (SKUTCH 1949, SLAGSVOLD 1982). Eltern können auch durch Verteidigung Prädatoren vom Nest fernhalten (REYER 1988, MeILVANG ET AL. 1997). Andererseits kann durch die Wahl des Neststandortes versucht werden, die Auffindbarkeit eines Nestes zu erschweren. FILLIATER ET AL. (1994) listen verschiedene Thesen auf, wie unterschiedliche Neststandorte selektiert werden können. Sie können in die Suche nach Extremen, d.h. besonders unwahrscheinlichen Standorten, die nicht im Suchschema der Prädatoren liegen, oder das "Verschwinden“ in der Menge gleichartig angelegter $\mathrm{Ne}$ ster eingeteilt werden. In vielen Fällen konnte gezeigt werden, dass es für eine Vogelart vorteilhafte Neststandorte gibt und eine Selektion solcher Standorte plausibel ist. Allerdings ist es nicht immer gelungen diesen Nachweis zu führen, was die Autoren (WILSON \& COOPER 1998) zu der Annahme verleitet, Nestprädation sei in diesen Fällen ein zufälliges Ereignis. SCHMIDT \& WHELAN (1999) weisen nachdrücklich darauf hin, dass Unvorhersagbarkeit auf Basis vorhandener Daten nicht gleichbedeutend ist mit Zufälligkeit. Sie legen dar, dass Neststandorte nicht einem einzelnen gerichteten Selektionsdruck unterliegen, sondern mehreren gegenläufigen, die sich ausgleichen können und eine Vorhersagbarkeit verhindern. Die Erforschung des Effektes verschiedener Prädatorentypen (MARTIN 1987) ist ein neuer Blickwinkel in der ökologischen Forschung (SIH ET AL. 1998). Bislang wurden im Hinblick auf Selektionsprozesse einfache Räuber-Beute Beziehungen untersucht. Auch wenn solche Wechselbeziehungen keine Ausnahme darstellen, sind die Systeme in der Regel komplexer: So z.B. in Nahrungsnetzen mit zahlreichen verschiedenen Prädatoren, die gleiche Nahrungsquellen nutzen, deren Zugang dazu aber unterschiedlich ist. Eier und Jungvögel sind eine solche Beute. Von vielen Vögeln und Säugetieren, die unterschiedliche Suchstrategien verwenden und damit unterschiedliche Feindvermeidungsstrategien selektieren, ist bekannt, dass sie Eier und Jungvögel fressen. Damit wäre Neststandortselektion bei Singvögeln ein Beispiel für den Effekt vieler Prädatoren auf eine Beute (SCHMIDT \& WHELAN 1999).

Die zweite Anpassungsstrategie ist den Effekt der Prädation zu reduzieren: Durch schnelle Kompensation wären die Vögel in der Lage, sehr bald nach einem Gelegeverlust ein neues Nest zu bauen und ein neues Gelege zu beginnen. Neben dieser Selektion auf schnelle Abfolge der Brutversuche wird eine Erhöhung der Sequenz 
der Brutversuche diskutiert, die durch eine Verminderung der Gelegegröße ermöglicht wird (SLAGSVOLD 1984). Diese Reduktion gestattet eine Risikoverteilung (,bet hedging") auf mehr Nester und erhöht die Wahrscheinlichkeit, dass einer der Brutversuche erfolgreich ist. Bislang einziger Beleg für diesen Mechanismus ist die Gelegegröße der Kohlmeise Parus major (JULLIARD ET AL. 1997), die negativ mit der Prädationsrate im Vorjahr korreliert. Auch die Länge der Brutsaison kann in diesem Zusammenhang gesehen werden: Je länger die Brutsaison dauert, desto mehr Brutversuche sind möglich und desto höher ist die Wahrscheinlichkeit eine erfolgreiche Brut durchzuführen (SLAGSVOLD 1982).

Die Prädationsrate hängt davon ab, ob in einem Gebiet viele Prädatoren gemeinsam oder spezialisierte Prädatoren alleine einen hohen Prädationsdruck erzeugen. Im Hinblick auf Elternverhalten und Neststandorte ist die Frage nach den Suchstrategien der Prädatoren maßgeblich. Wenn Prädatoren nicht auf Altvögel achten, sondern nachts auf Nahrungssuche sind oder Flächen systematisch absuchen, gibt es vermutlich keine Selektion heimlichen Verhaltens. Wenn es sich um Prädatoren handelt, die vertrieben werden können, kann Verteidigung eine Strategie sein. Auch die Selektion des Neststandortes ist von den Prädatoren abhängig. Beobachtungen auf Inseln zeigen, dass in Abwesenheit von Nesträubern leicht auffindbare Neststandorte gewählt werden, die dafür vor anderen Störungen sicher sind (ROBINET ET AL. 1998). Sobald dann Prädatoren auftreten, kann dies zum Aussterben von Vogelarten führen (SAVIDGE 1987, ConRY 1988, RoBINET ET AL. 1998). Entfällt die Möglichkeit die Prädationsrate zu reduzieren, können schnelle Nachgelege oder Risikoverteilung zur Kompensation unvermeidbarer Verluste selektive Vorteile bieten.

Die Notwendigkeit Prädatoren spezifizieren zu können wurde spätestens von MARTIN (1987) festgestellt. Verschiedene methodische Ansätze sind seitdem entwickelt worden, um einerseits Nestprädatoren am Nest zu bestimmen (MAJOR 1991). Andererseits wurde, ausgehend von Beobachtungen, dass Prädationsraten zwischen einzelnen Untersuchungsflächen schwanken können, versucht, indirekt auf mögliche Prädatoren zu schließen (ANDRÉN 1992). Letztgenannter Ansatz ermöglicht es, landschaftsbezogene Aspekte zu bearbeiten. In diesem Zusammenhang wird häufig die Frage diskutiert, welche ökologischen Auswirkungen anthropogene Veränderungen in der Landschaftsstruktur, vor allem Fragmentierung der Landschaft, haben können (PATON 1994, HARTLEY \& HUNTER 1998). Bei Vogelarten, die entlang von Waldrändern brüten, wird befürchtet, dass dort durch erhöhte Prädation unnatürlich hohe und 
nicht mehr kompensierbare Brutverluste auftreten können (GATES \& GYSEL 1978). Die Betrachtung einzelner Populationen in der Landschaft erlaubt auch Unterschiede in der Gelegegröße zu untersuchen und dabei Rückschlüsse auf mögliche Feindvermeidungsstrategien zu ziehen. Die Bestimmung der Prädatoren ist durch den Nachweis von Spuren am Nest oder durch Fotofallen an Kunstnestern versucht worden. Die methodischen Einschränkungen liegen auf der Hand (MAJOR \& KENDAL 1996, KING ET AL. 1999): Verteidigung durch Altvögel kann an Kunstnestern nicht berücksichtigt werden, Spurensuche an echten Nestern dagegen beinhaltet immer eine gewisse Unsicherheit, da nicht zweifelsfrei nachgewiesen werden kann, ob der Verursacher der Spuren auch den Nestinhalt gefressen hat und je nach methodischem Ansatz werden ganze Prädatorengruppen ausgeschlossen.

Eine Methode, die mit einem Minimum an Einschränkungen auskommt, ist die kontinuierliche Videoüberwachung aktiver Nester (PIETZ \& GRANSFORS 1998). Da diese Methode bis vor kurzem noch sehr teuer war, wurden nur einzelne Geräte eingesetzt und zumeist auch nur für Verhaltensbeobachtungen. Seitdem die Miniaturisierung elektronischer Bauteile die Produktion billiger Platinenkameras gestattet, können Videosysteme in größerer Zahl parallel eingesetzt werden. Bislang liegen nur wenige Untersuchungen vor, die mit Videoüberwachungen Nestprädatoren bestimmt haben (Brown ET AL. 1998, ThOMPSON ET AL. 1999, PIETZ \& Gransfors 2000). Es gibt bislang auch keine Publikationen, die auf Basis von Videoüberwachungen, also bei genauer Kenntnis der Prädatoren aktiver Nester, die voranstehenden Fragestellungen bearbeitet hätten.

\subsection{Fragestellungen}

An der Max-Planck-Forschungsstelle für Ornithologie, Andechs \& Radolfzell, Vogelwarte Radolfzell, wurde in einer Pilotstudie die Anwendbarkeit von Videokameras zur Überwachung von Singvogelnestern erprobt (SELL 1998). Auf Grundlage dieser Erfahrungen wurde die vorliegende Arbeit angefertigt, die im Kern die Bestimmung von Nestprädatoren der Mönchsgrasmücke durch Videoüberwachungen, die Erfassung brutbiologischer Parameter sowie die Vermessung von Neststandorten in Südwestdeutschland und auf Mallorca (gefördert vom DAAD) zur Aufgabe hatte. Die Auswertung der Nestfunde von Mitarbeitern der Vogelwarte Radolfzell aus demselben Gebiet seit 1981 ermöglicht darüber hinaus die Analyse zeitlicher und räumlicher Zusammenhänge zwischen Prädation und Lebenszyklusmerkmalen. 
In dieser Arbeit soll der Frage nachgegangen werden, welche evolutiven Strategien die Mönchsgrasmücke im Hinblick auf Gelegeverluste entwickelt hat. Die beiden Teile der Untersuchung, nämlich Erfassung von Prädatoren und Auswertung von Nestfunden der Vogelwarte, bieten die Möglichkeit, die Fragen gemäß Abb. 1 zu behandeln:

1. Welches Verhalten zeigen Mönchsgrasmücken gegenüber Prädatoren?

2. Gibt es Neststandortselektion durch Prädation?

3. Kann die Mönchsgrasmücke Gelegeverluste kompensieren?

4. Gibt es Hinweise auf eine Risikoverteilung?

Zu 1.: Die Frage nach Elternverhalten und möglichen Anpassungen an Prädation wurde mit Videoüberwachungen untersucht. Die Analyse von Prädationsmustern aus Nestdaten und die Kenntnis der Prädatoren ermöglichen eine Aussage darüber, welche Anpassungen zu erwarten sind. Der Vergleich von Videoüberwachungen und Fotofallen, d.h. verteidigten und nicht verteidigten Nestern, kann darüber hinaus Hinweise darauf geben, ob die Mönchsgrasmücke in der Lage ist, ihre Nester gegen Prädatoren zu verteidigen.

Zu 2.: Es wurde untersucht, ob eine Selektion auf vorteilhafte Neststandorte nachweisbar ist. Nach Kenntnis der Prädatoren wurde diese Untersuchung im Hinblick auf mögliche Effekte mehrerer Prädatoren wiederholt. Die Studie auf Mallorca wurde durchgeführt, um Neststandortselektion und Prädation in einem Gebiet zu untersuchen, in dem Rabenvögel als eine mögliche Prädatorengruppe fehlen.

Zu 3.: Die Frage nach der Kompensation von Gelegeverlusten konnte mit Hilfe von Brutdaten aus der Volierenanlage der Vogelwarte bearbeitet werden. Die Analyse von Nestdaten der Vogelwarte gestattete Aussagen über Veränderungen in der Brutphänologie.

Zu 4.: Die Nestdaten der Vogelwarte wurden darüber hinaus für die Frage nach einer Risikoverteilung ausgewertet. Unterschiede zwischen den Untersuchungsflächen wurden analysiert, mit landschaftsbezogenen Parametern in Verbindung gebracht und Effekte der Gelegegröße dargestellt. Mögliche Konsequenzen der Prädationsrate auf die Reproduktion im Folgejahr wurden untersucht.

Wie in der Einleitung angesprochen, wird vielfach diskutiert, ob Nestprädation infolge der Fragmentierung der Landschaft vermehrt auftritt und zu Populationsrückgängen 
bei Singvögeln führt (BAUER \& LEY 1994). Besonders Rabenvögel werden als ursächlich angesehen und die Kontrolle ihrer Populationen gefordert (DEUTSCHER JAGDSCHUTZVERBAND E.V. 1997). Da diese Studie erstmals in Mitteleuropa systematisch Prädatoren einer gebüschbrütenden Vogelart erfasst, wurden die Daten auch im Hinblick auf diese Problematik diskutiert. 


\section{Die Mönchsgrasmücke als Forschungsgegenstand}

Die Mönchsgrasmücke ist für die Bearbeitung der vorgegebenen Fragestellung besonders gut geeignet. Sie ist eine der häufigsten Arten im Untersuchungsgebiet (HEINE ET AL. 1999, WIDMER 1999) und brütet zudem in hohen Dichten mit bis zu 8 Brutpaaren pro ha (BAIRLEIN 1978, BERTHOLD \& QUERNER 1984). Die Nester sind bei systematischer Suche vergleichsweise leicht zu finden, entscheidend um auch Daten aus frühen Neststadien zu erhalten. Die Brutsaison reicht von Mitte April bis Anfang August. Es ist somit gewährleistet, dass auch ein einzelner Bearbeiter ausreichend Nester findet.

Wichtiger noch als diese praktischen Erwägungen sind inhaltliche. Die Mönchsgrasmücke brütet in allen Waldtypen, sofern Deckung bietende Strukturen zur Nestanlage vorhanden sind (BERTHOLD 1978). Die Nesthöhe in Mitteleuropa schwankt zwischen null und sechs Metern ( BERTHOLD ET AL. 1990), die Anzahl genutzter Strukturen ist sehr groß. Die Mönchsgrasmücke integriert damit die Ansprüche vieler im Untersuchungsgebiet vorkommender Singvogelarten, denn bei der Nestersuche werden regelmäßig Nester von Amsel Turdus merula, Singdrossel T. philomelos, Gelbspötter Hippolais icterina, Gartengrasmücke $S$. borin, Dorngrasmücke $S$. communis, Zilpzalp Phylloscopus collybita, Zaunkönig Troglodytes troglodytes, Heckenbraunelle Prunella modularis und Goldammer Emberiza cirtinella, also vieler gebüschbrütender Vogelarten, gefunden. Die enorme Bandbreite der Neststandortselektion lässt erwarten, dass kein gerichteter Selektionsdruck vorliegt und die Mönchsgrasmücke damit

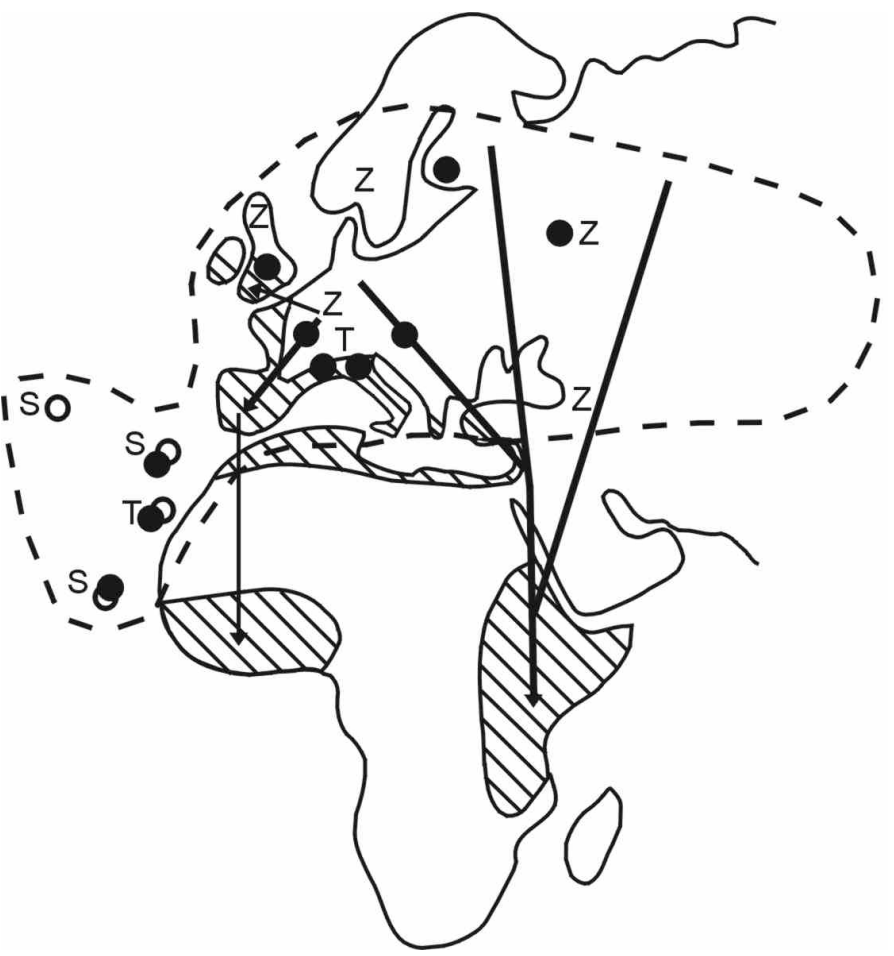

Abb. 2: Schematische Darstellung der Lebensformen der Mönchsgrasmücke. Z: ausschließlich Zugvögel, T: Teilzieher, S: Standvögel, dicke Pfeile: Haupt-, dünne Pfeile: Nebenzug-richtungen, gestrichelt: äußere Grenze der Brutverbreitung, schraffiert: Überwinterungsgebiete (aus BERTHOLD 2000 nach BERTHOLD 1999). 
ein geeignetes Objekt für die Untersuchung von Effekten vieler Prädatoren ist.

Die Mönchsgrasmücke hat ein großes Verbreitungsgebiet, das von den Kapverdischen Inseln bis hinter den Ural reicht (Abb. 2). Untersuchungen haben große Unterschiede in vielen ökologischen Parametern zwischen den einzelnen Populationen gezeigt. Inselpopulationen im Mittelmeer und Atlantik ziehen nicht, südeuropäische Populationen sind Teilzieher, schon süddeutsche Mönchsgrasmücken verlassen im Herbst das Brutgebiet vollständig. Mit der geografischen Höhe verlängern sich die Zugstrecken und Ankunftstermine im Brutgebiet. Auch in brutbiologischen Parametern gibt es viele Unterschiede: Frühester Legebeginn ist auf den Kanaren Mitte März, in Südfrankreich und der Schweiz Anfang April, in Süddeutschland Mitte April und in den nördlichsten Verbreitungsgebieten Mitte Mai. Die Gelegegröße schwankt zwischen drei und sechs Eiern in relativ engen Grenzen. Es gibt saisonale und latitudinale Schwankungen, die eine genetische und/oder phänotypische Variation nachweisen, was für die Untersuchung von Änderungen dieses Parameters eine Grundvoraussetzung ist. Die Art verfügt also auch in diesen Parametern über eine große Plastizität. Populationen auf Inseln unterscheiden sich auch in anderen Voraussetzungen von Festlandpopulationen. So kommen Rabenvögel, die als die Hauptnestprädatoren gelten, auf den Kanaren, auf Madeira und den Balearen nicht vor (GLUTZ VON BLOTZHEIM \& BAUER 1993). Diese Beobachtungen bieten die Voraussetzung für überregionale Vergleiche, wie sie in dieser Untersuchung durchgeführt wurden. 


\section{Untersuchungsgebiete und Methoden}

Für die vorliegende Untersuchung wurden verschiedene Datenquellen verwendet. Zum einen wurden in den Jahren 1999, 2000 und 2001 in der Umgebung um Radolfzell am westlichen Bodensee, Baden-Württemberg, Nester der Mönchsgrasmükke zur Video- und Fotofallenüberwachung gesucht. In den Monaten April und Mai 2001 konnte zusätzlich eine Vergleichsstudie auf Mallorca durchgeführt werden. Von allen Nestern wurden Neststandortparameter erhoben (3.1). Zum anderen wurden Daten von Nestfunden der Mönchsgrasmücke von der Forschungsstelle für Ornithologie ausgewertet, die im Rahmen verschiedener Untersuchungen seit 1981 erhoben, im Hinblick auf Brutbiologie bislang noch nicht ausgewertet wurden (3.2). Die Methoden werden getrennt beschrieben. Da sich die Ergebnisse ergänzen, wird diese Trennung im Ergebnisteil aufgegeben und es wird auf die jeweilige Datenquelle verwiesen. Eine zusätzliche Datenquelle bezieht sich ausschließlich auf die Nachlegekapazität der Mönchsgrasmücke, die aus Volierendaten bestimmt wurde (3.3). Details zur Auswertung, die in den Methoden nicht erörtert sind, erscheinen im Ergebnisteil. Alle statistischen Auswertungen wurden in JMP 4.01, SAS Institute durchgeführt. Für einige nicht signifikante Ergebnisse wurde in JMP eine Poweranalyse durchgeführt um auszuschließen, dass ein vorhandener Effekt durch zu geringe Stichprobengröße nicht berücksichtigt wird (JOHNSON 1999).

\subsection{Prädationsstudie}

\subsubsection{Untersuchungsflächen}

\subsubsection{Südwestdeutschland}

Für die Videoüberwachungen wurden in der Umgebung der MaxPlanck Forschungsstelle für Ornithologie in der Nähe der Stadt Radolfzell am westlichen Bodensee, Baden-Württemberg (Abb. 3),

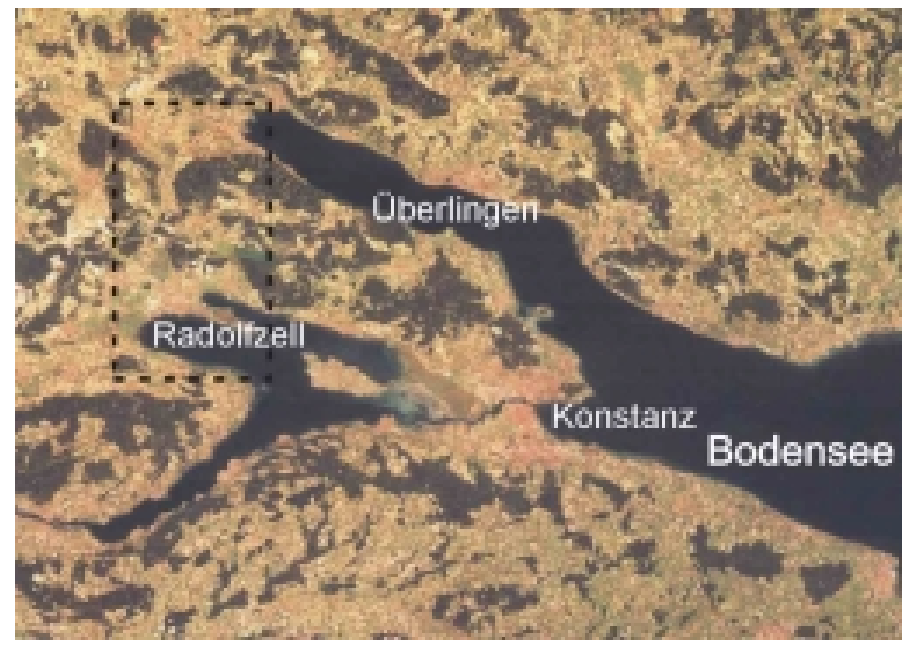

Waldstücke und -ränder ausge- Abb. 3: Der westliche Bodenseeraum mit der Lage der wählt, die nach eigener Anschau- Untersuchungsgebiete bei Radolfzell (Kasten). 


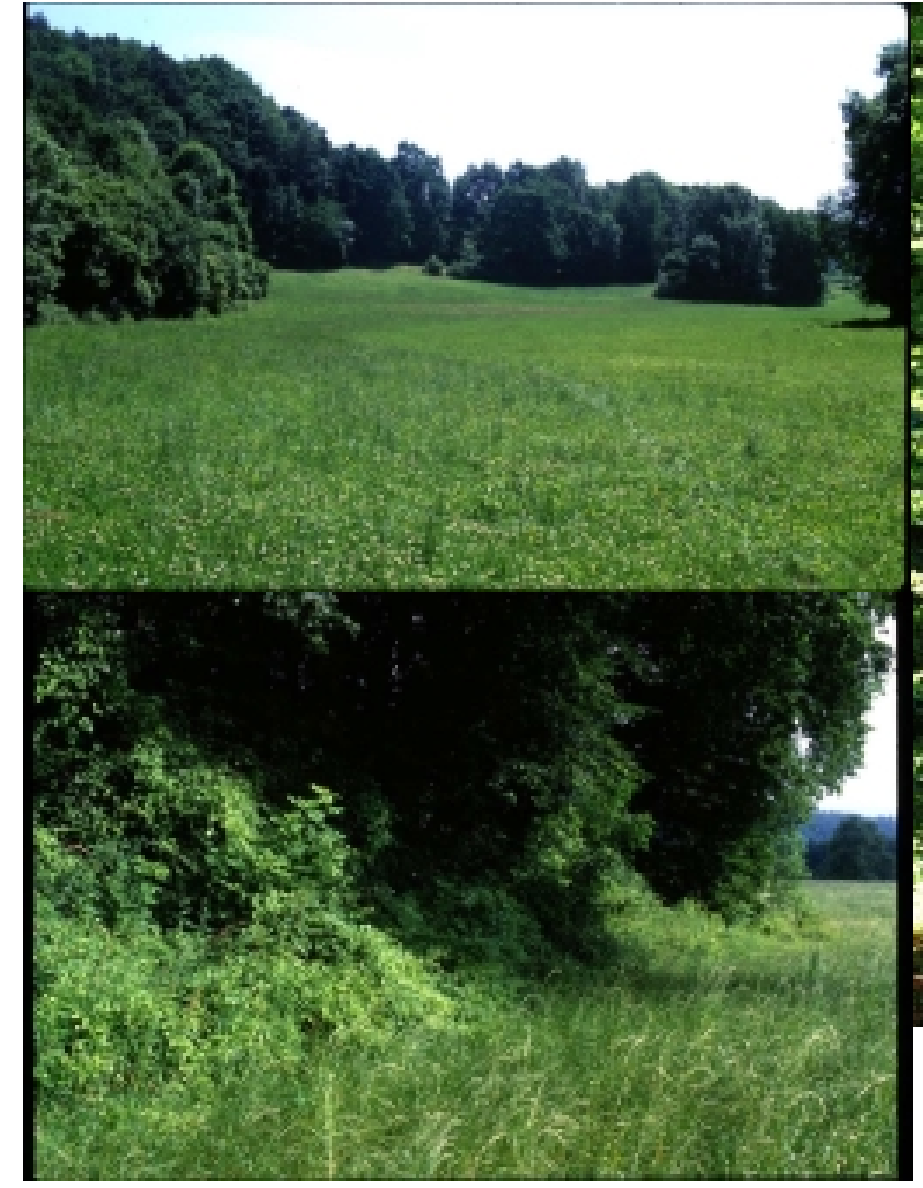

Abb. 4: Bruthabitate der Mönchsgrasmücke in SW-D. Mönchsgrasmücken boten (Abb. oben: Hecken in einem Tal, unten: Waldrand, rechts: Lichtung.
4). Dies waren im Allgemeinen Mischwälder an den Hängen des

Bodanrücks, dominiert durch Buche Fagus sylvatica. Im Inneren fanden sich kleine Lichtungen oder Naturverjüngungsflächen, auf denen Brombeeren Rubus ssp., Brennnesseln Urtica dioica, Jungbuchen und andere niedrigere Strukturen auf gröBerer Fläche Nestmöglichkeiten boten. An den Waldrändern fanden sich zahlreiche holzige Pflanzen wie Weißdorn Crategus spec. und Schwarzdorn Prunus spinosa, Hartriegel Cornus sanguinea, Schneeball Viburnum lantana, und Efeu Hedera helix, die ebenfalls zur Nestanlage geeignet sind (BERTHOLD ET AL. 1990).

Das Gebiet wurde möglichst groß gewählt, um lokale Besonderheiten erkennen zu können und ein möglichst breites Spektrum potentieller Prädatoren zu erhalten (Abb. 6). Gesucht wurde in direkter Umgebung von Schloss Möggingen, oberhalb des Ziegelhofes (nördlicher Rand des Alten Bohls), Hirschbrunnen, Durchenberg, Böhlerberg, Nusshalde, Oberer Brandbühlwald, Wolfsgrund und Kuhbühl sowie der Schlosshalde südlich von Wahlwies. Die Strecke regelmäßig abgesuchter Waldränder belief sich auf etwa $9 \mathrm{~km}$. Insgesamt wurden ca. 40 ha regelmäßig nach Nestern 
durchsucht, die sich auf einer Fläche von etwa 2000 ha verteilten. Kriterium für die Flächenauswahl war nach der Abundanz der Mönchsgrasmücken die Erreichbarkeit der Nester. Aufgrund der Notwendigkeit Geräte und Akkus zu transportieren, wurden möglichst geringe Distanzen zu Fahrwegen eingehalten, was bei der hohen Wegdichte im Untersuchungsgebiet unproblematisch war.

\subsubsection{Mallorca}

Die Mönchsgrasmücke besetzt im Mittelmeerraum laut Literaturangaben (BERTHOLD ET AL. 1990) bereits Ende Februar/Anfang März ihre Reviere und zeigt zu dieser Zeit hohe Gesangsaktivitäten. Daher wurde nach der Ankunft Anfang April mit der Auswahl der Untersuchungsgebiete begonnen. Für einen Vergleich mit den mitteleuropäischen Daten war es erforderlich, strukturell vergleichbare Gebiete zu finden. Zwei Flächen wurden an der Nordostküste der Insel ausgewählt (Abb. 5), die in der Vegetationsstruktur den süddeutschen Wäldern sehr ähnlich waren. Die erste Fläche mit 30 ha lag am Fuße des Puig Sant Marti und war durch ein weites Tal mit angrenzenden Hängen gekenn-

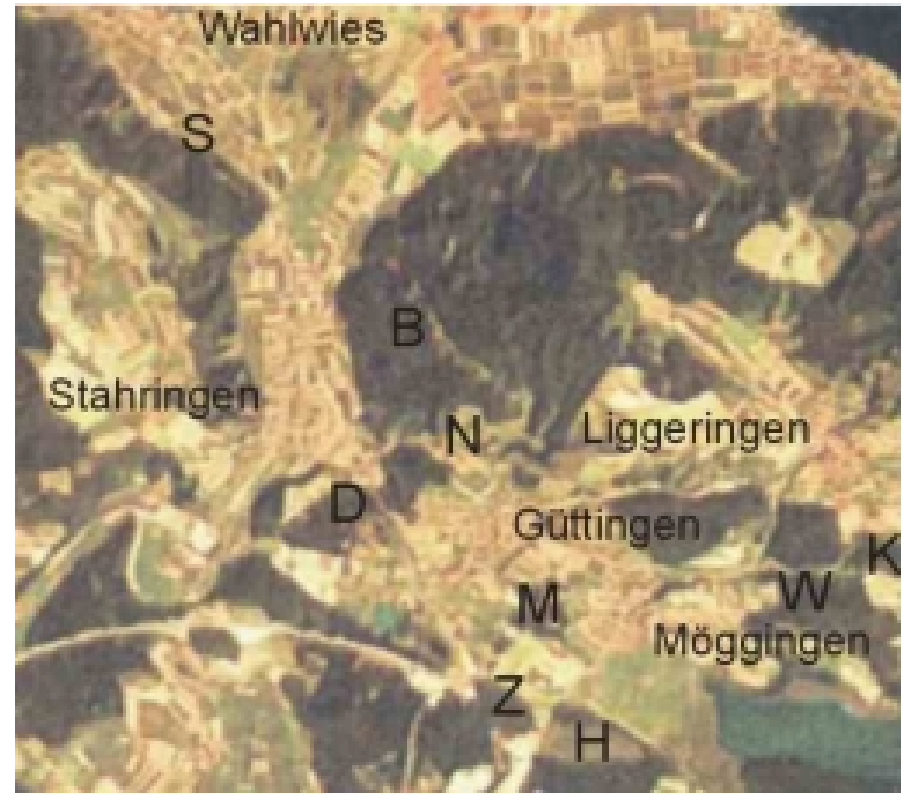

Abb. 6: Die Untersuchungsflächen in der Umgebung der Dörfer Wahlwies, Stahringen, Liggeringen, Güttingen und Möggingen (S: Schlosshalde, B: Böhlerberg, N:

Nusshalde, D: Durchenberg, M: Schloss Möggingen, Z:

Ziegelhofweg, H: Hirschbrunnen, K: Kuhbühl, W:

Wolfsgrund).

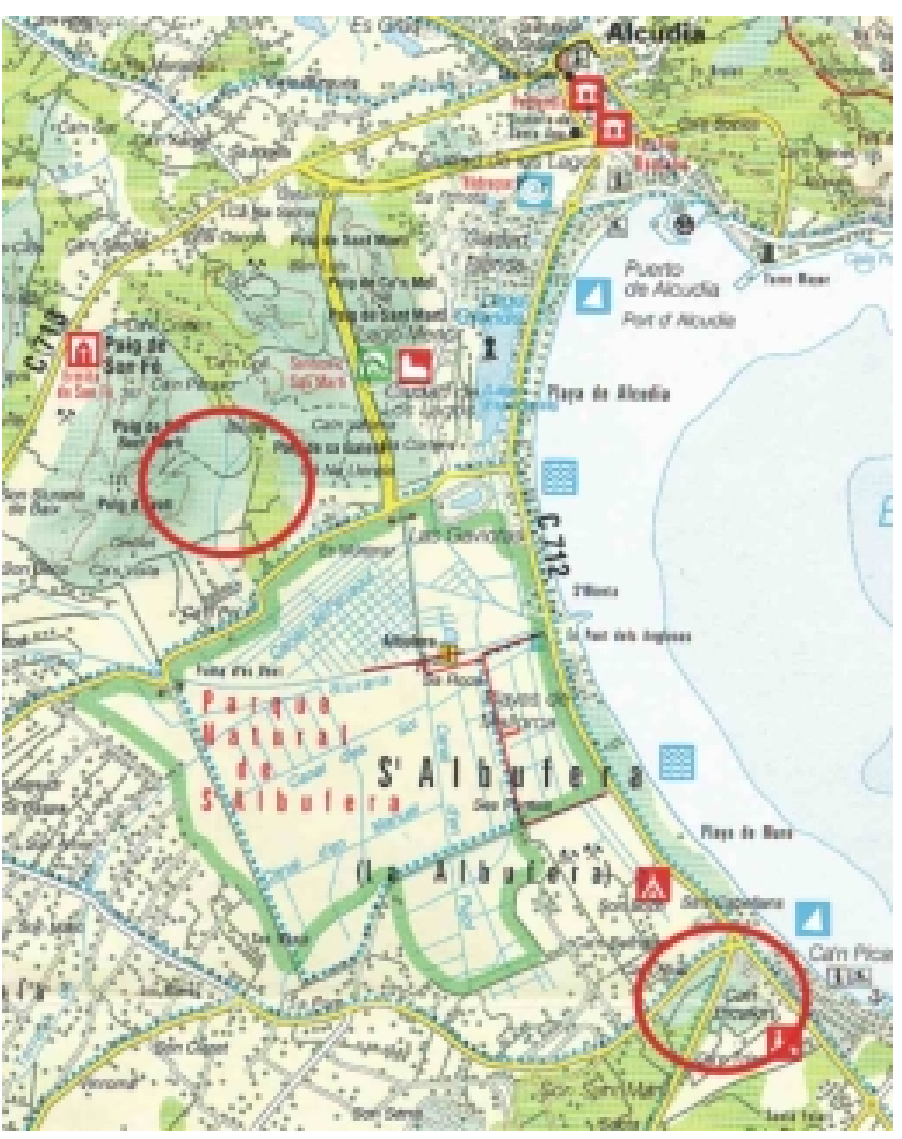

Abb. 5: Lage der Untersuchungsflächen in der Bucht von Alcudia im Nordosten Mallorcas. 
zeichnet (Abb. 7).

Die Hänge waren mit Aleppo Kiefern Pinus halepensis bewachsen. Dieser Baum ermöglicht mit seinem lichten Kronendach im Unterwuchs eine dichte Strauchschicht aus Mastix Pistacea lentiscus, Steineiche Quercus ilex, Steinlinde Phillyrea spec. Neben diesen Sträuchern und Jungbäumen gab es eine Vielzahl krautiger Pflanzen wie Kletten-Krapp Rubia peregrina, Stechginster Asparagus acutifolius, verschiedene Cistrosenarten Cistus spec., Brombeere Rubus spec. und Myrte Myrtus communis. Die Talsohle bestand aus waldfreien Macchiebereichen, die für eine Besiedlung durch die Mönchsgrasmücke nicht in Frage kommen. Die zweite etwa 26 ha große Fläche lag im südlichen Teil des Nationalparks S'Albufera auf einer Düne hinter dem Strandwall zur Bucht von Alcudia und bestand ebenfalls aus einem immergrünen Wald mit Aleppo Kiefern und reichem Unterholzanteil. Auch diese Fläche war kleinräumig von offenen Macchieflächen durchzogen, die wie am Puig Sant Marti etwa die Hälfte der Gesamtfläche ausmachten.

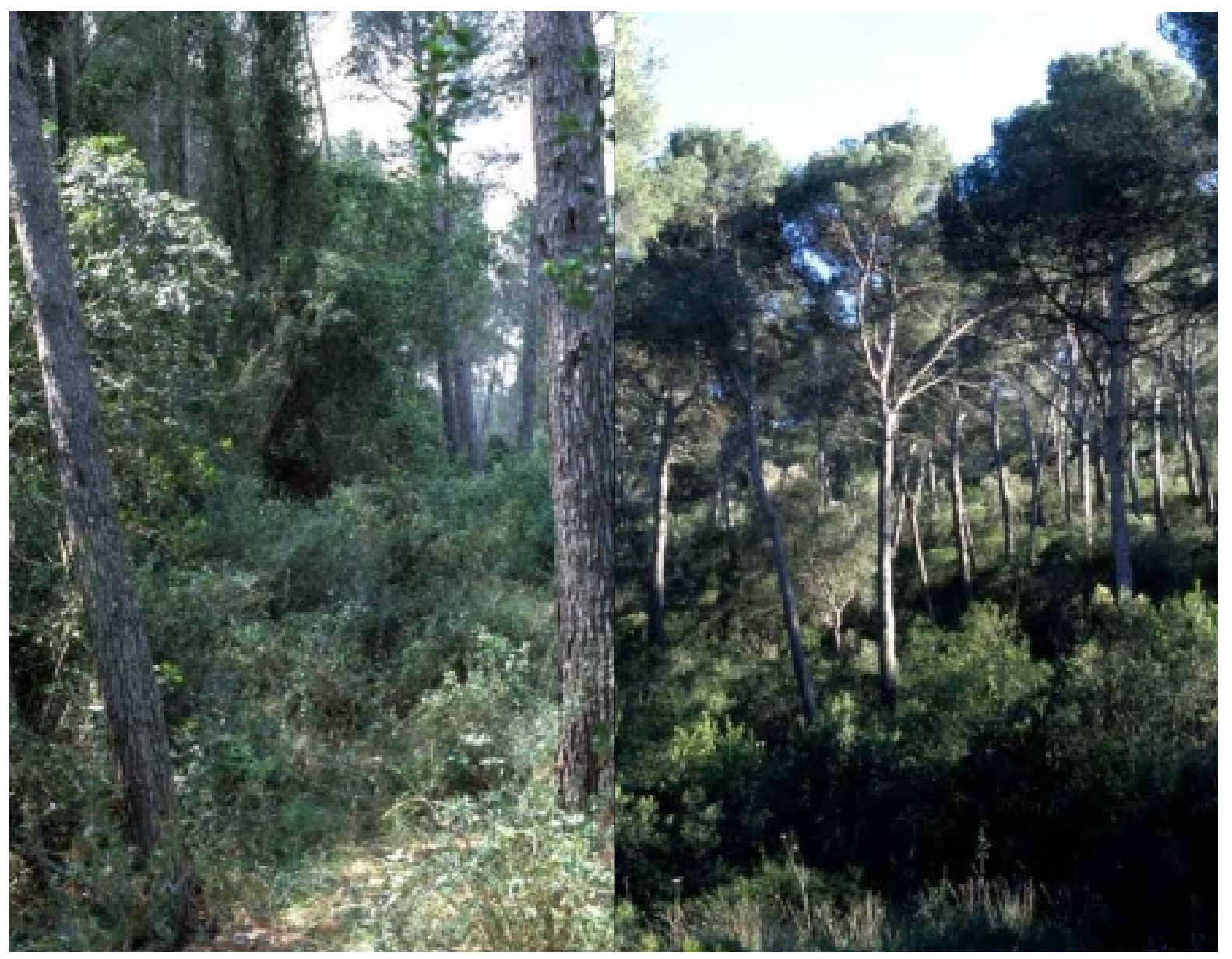

Abb. 7: Bruthabitate der Mönchsgrasmücke auf Mallorca. 


\subsubsection{Nestersuche}

Nester der Mönchsgrasmücke sind vergleichsweise einfach zu finden, weshalb sich eine systematische Suche in geeigneten Strukturen lohnt (BERTHOLD 1976, MARTIN \& GEUPEL 1993). Auffliegende Altvögel sind natürlich ein hilfreicher Hinweis auf ein Nest, aber das alleinige Beobachten von Altvögeln, die etwa Nestmaterial oder Nahrung eintragen, ist nicht effektiv genug. Eine systematische Suche hat den Vorteil, dass Nester recht früh in der Lege- oder Brutphase gefunden werden können, wenn die einzelnen Flächen regelmäßig abgesucht werden. Selbst Nester im Bau oder frisch fertiggestellte Nester werden auf diese Weise entdeckt. So wurden in den ausgewählten Flächen entlang der Waldränder und im Inneren alle in Frage kommenden Strukturen durchsucht. Dazu zählten Brennnesselfelder, Brombeerflächen, Büsche und Sträucher, Waldrebe, Efeu und Hopfen entlang von Waldrändern und vorgelagerten Hecken und auf Verjüngungsflächen im Waldinnern. Darüber hinaus nutzt die Mönchsgrasmücke gelegentlich auch Stockausschläge von Laubbäumen, junge Fichten oder herabhängende Äste von älteren Fichten, herabgefallene trockene Fichtenäste und selten auch Farne (BERTHOLD ET AL. 1990). Nach einem Nestfund wurde der Standort im Feld markiert und in einer Lageskizze notiert. Eizahl, Bebrütungszustand oder Alter von Jungen wurden festgestellt und anschließend eine Videokamera installiert. Nester, die nicht mit Video überwacht werden konnten, wurden jeden zweiten Tag kontrolliert, um den Bruterfolg zu erfassen.

\subsubsection{Videoüberwachung von Nestern der Mönchsgrasmücke}

Nach dem Fund eines Nestes wurde zur Dauerüberwachung des Brutgeschehens in Nestnähe ein Videosystem installiert. Da solche Systeme im Freiland bislang nur wenig zum Einsatz gekommen sind, wird diese Methode im Folgenden ausführlich beschrieben. Es wurden zwei unterschiedliche Kameras benutzt, die jeweils Vor- und Nachteile haben, auf die ebenfalls im Einzelnen eingegangen wird.

\subsubsection{Videoüberwachung im Freiland}

Das hochauflösende System (HR für High Resolution) bestand aus einer infrarotsensitiven s/w-Kamera mit 540 Videolinien, die mit einem lichtstarken Zoomobjektiv mit veränderbarer Blende ausgestattet wurde, um die Entfernung der Kamera zum Nest zwischen $1 \mathrm{~m}$ und $5 \mathrm{~m}$ wählen zu können. Die Kamera befand sich in einem wasserdichten Spezialgehäuse, das auf einem handelsüblichen Stativ befestigt werden 

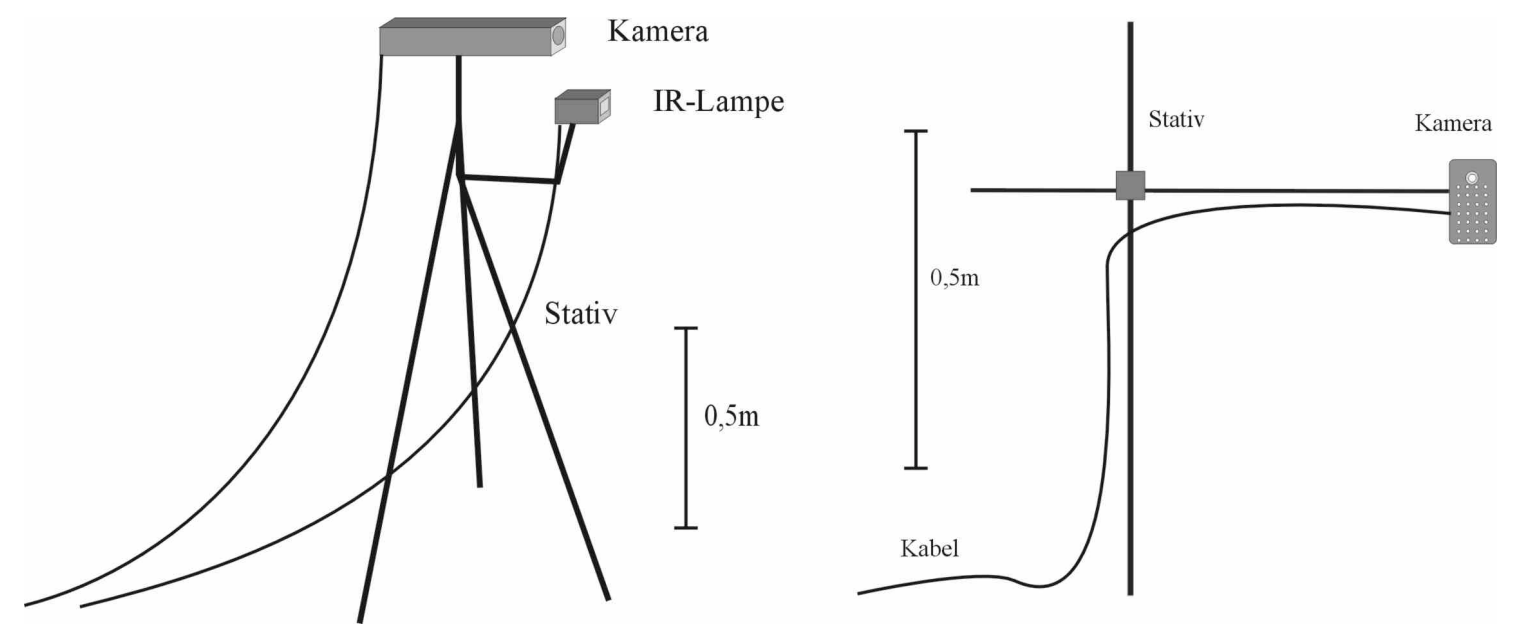

Abb. 8: Schema der verwendeten Videosysteme (links: HR, rechts: LB).

konnte. Da Nesträuber auch nachts an Nestern auftreten können, wurde mit einem zusätzlichen Stativarm eine Infrarothalogenlampe befestigt, die leicht auf das Nest auszurichten war. Kamera und Lampe wurden über separate Kabel mit Strom versorgt, ein weiteres Kabel leitete das Videosignal an den Rekorder (Abb. 8).

Das System mit der einfachen Platinenkamera (LB für Low Budget) bestand aus einer s/w-Platinenkamera mit 380 Videolinien und einer einfachen Festoptik mit $30^{\circ}$ Öffnungswinkel. Dadurch war eine Entfernung zwischen $70 \mathrm{~cm}$ (min. Fokusentfernung) und $150 \mathrm{~cm}$ (Größe auf dem Bild) vom Nest vorgegeben. Die Kamera wurde zusammen mit einem Infrarotdiodenscheinwerfer aus 28 Dioden in einem PEStandardgehäuse installiert, das mit den erforderlichen Anschlüssen versehen wurde. Das Gehäuse wurde über eine selbstgefertigte Kupplung an einer Netzstange befestigt, die einfach am Nest in den Boden gesteckt werden konnte (Abb. 8).

Videorekorder, Monitor und die Stromversorgung, die über $12 \mathrm{~V}$ Bleigelakkus gewährleistet wurde, befanden sich in einer Transportkiste, die in ca. $25 \mathrm{~m}$ Entfernung

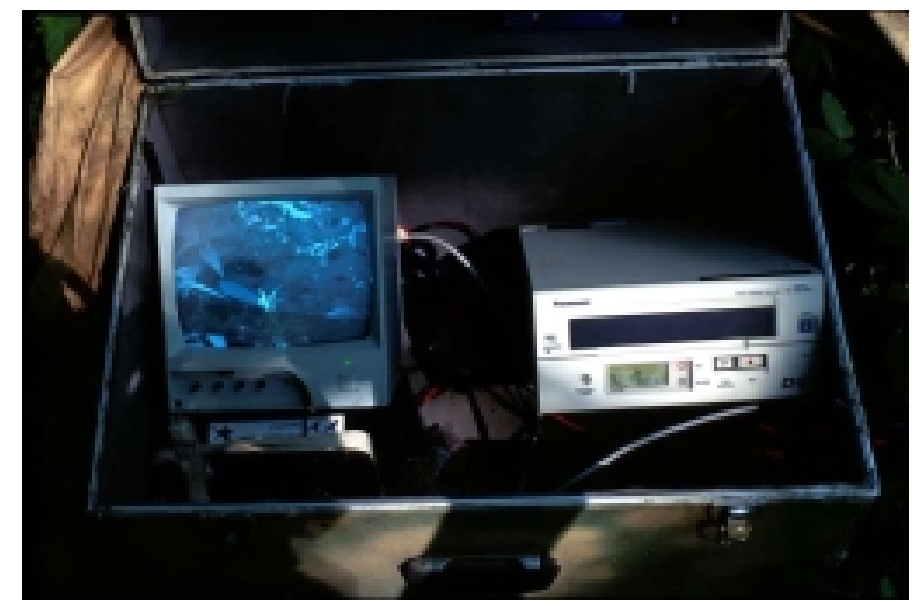

Abb. 9: Transportkiste mit Rekorder, Monitor und Akku. von der Kamera versteckt wurde (Abb. 9). Kamera und Rekorder haben bei $12 \mathrm{~V}$ zusammen einen Strombedarf von ca. $16 \mathrm{~W}$, die in 24 Stunden Akkus mit 37 Ah erforderten. Da Bleigelakkus ebenso wie normale Autobatterien nur ca. $80 \%$ ihrer Leistung freisetzen, sind Akkus mit etwa 43 Ah erforderlich, um einen 24 Stunden- 
Dauerbetrieb zu ermöglichen. Die nächtliche Beleuchtung der HR-Systeme benötigt $20 \mathrm{~W}$, die in 10 Stunden ca. 27 Ah erforderten. Da die Halogenlampe nur nachts zugeschaltet wurde, konnten die Systeme tagsüber 14 Stunden mit einem 33 Ah-Akku betrieben werden, nachts wurden die HR-Systeme und die Lampe gemeinsam über einen 43 Ah-Akku versorgt. Es waren also zwei Akkuwechsel täglich erforderlich. Da der Diodenscheinwerfer nur 1,3 W benötigt, musste er nicht separat zugeschaltet werden, sondern lief im Dauerbetrieb. Ein LB-System wurde 24 Stunden mit einem 43 Ah-Akku versorgt.

Verwendet wurden Time-Lapse-Videorekorder, die es erlauben die Betriebsdauer für ein 180 min VHS-Band zwischen 3, 6, 12 und 24 h zu wählen. Neben dem Panasonic AG-1070 DC für ca. € 1200 (Preise Stand Frühjahr 2000) kam der Sanyo TLS 224P für ca. $€ 700$ zum Einsatz. Eine Auswahl bestand lediglich zwischen diesen beiden Geräten, da Videorekorder normalerweise nicht mit $12 \mathrm{~V}$ betrieben werden und ein Umbau sehr teuer ist. Es gibt zahlreiche Unterschiede zwischen den Geräten, die für diese Untersuchung nicht maßgeblich waren. So bleibt nach den Erfahrungen dieser Studie zu sagen, dass die robusteren Panasonic Geräte häufiger ausfielen als die Sanyo Geräte.

Die Kosten für ein HR-System lagen beträchtlich über denen eines LB-Systems. Neben der verwendeten Kamera mit Optik im Gehäuse und der Lampe im Gehäuse (ca. $€ 1300$ ) war der Einsatz eines robusten und wetterfesten Stativs mit Stativarm erforderlich (ca. € 500). Zu den höheren Kosten trug auch der zusätzliche Akkuwechsel bei, der bei diesem System notwendig ist, so dass zusätzlich gefahrene Kilometer und erhöhter Zeitaufwand erheblich zu Buche schlugen. Kamera mit Beleuchtung und Stativ des LB-Systems kosteten ca. $€ 150$, wenn man die Einzelteile selber integrierte. Die restliche Ausrüstung (Kiste, Akkus, Ladegerät, Monitor, Kabel, Anschlüsse etc.) beliefen sich auf ca. $€ 750$, wobei Einsparungen von ca. $€ 250$ möglich wären, wenn man Akkus geringerer Qualität und einen weniger guten Monitor einsetzt (oder sich auch bei mehreren Systemen auf nur einen beschränkt). Die Anschaffungskosten für ein HR-Set liegen bei Verwendung eines Sanyo Rekorders bei ca. $€$ 3300 , die für ein LB Set bei ca. $€ 1600$.

\subsubsection{Aufstellen der Kameras}

1999 waren fünf HR-Systeme verfügbar, im Jahr 2000 konnten dazu fünf LBSysteme angeschafft werden. Zur Installation am Nest wurde die Kamera zunächst 
mit allen erforderlichen Anschlüssen und Kabeln ausgestattet, damit jeweils ein einmaliges Aufsuchen des Nestes ausreichte. Die Kameras wurden zumeist von der Innenseite des Nestbusches installiert, damit der Blick auf das Nest ohne größere Veränderungen möglich war (Abb. 10, Abb. 11). Dennoch mussten häufig einzelne Blätter oder trockene Äste entfernt werden, besonders bei Nestern, die in geringer Höhe in Brombeerbüschen oder Brennnesseln angelegt waren. Die Kiste wurde in einem Abstand von ca. $25 \mathrm{~m}$ positioniert und so jedes Nest bis zum Ausfliegen der Jungen oder einem Prädationsereignis durchgehend überwacht. Akkus wurden jeweils bei Sonnenaufgang und -untergang zusammen mit den Videobändern gewechselt.

\subsubsection{Erfahrungen beim Betrieb}

Beide Systeme wurden im 24-Stundenbetrieb eingesetzt. Das Aufstellen der HRKameras dauerte jeweils etwa 30 min, weil neben der Kamera, bei der eine genaue Fokussierung sowie ein Ausrichten des Halogenstrahlers erforderlich war, auch das Stativ eingerichtet werden musste. Im Normalfall war auch noch ein Nachjustieren der Strahlerausrichtung nach Einbruch der Dunkelheit erforderlich, was zusätzliche Zeit und einen weiteren Besuch am Nest erforderte. Das Aufstellen der LB-Systeme dauerte nur die Hälfte der Zeit und ein Nachjustieren der Lampe war nicht erforderlich, da die Beleuchtung im Kameragehäuse integriert war. Abhängig von der Lauf-

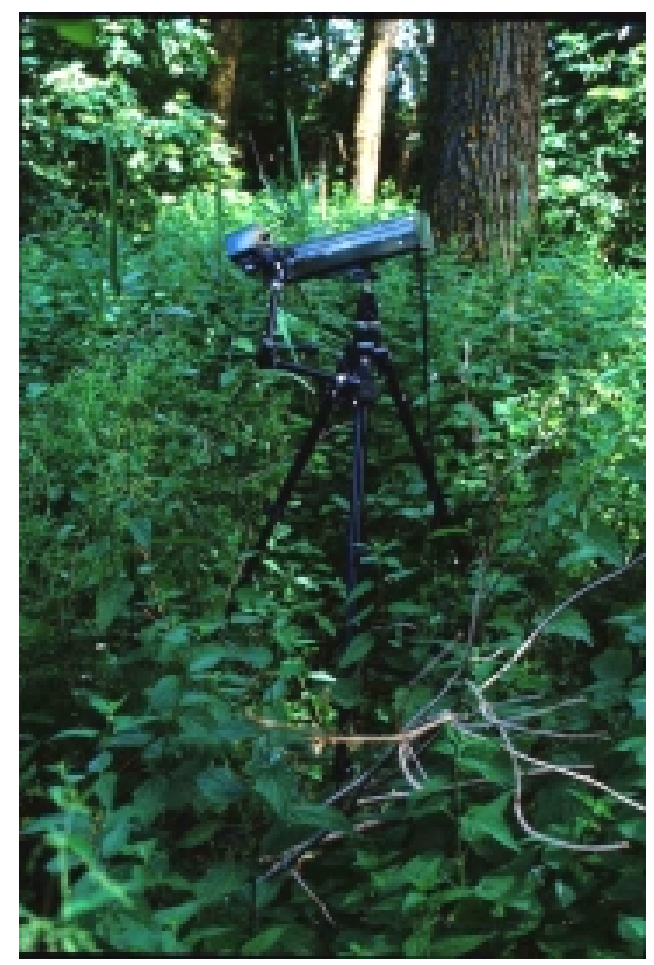

Abb. 10: HR-Kamera an einem Nest. strecke vom Auto zur Kamera und der Entfernung der Kamerastandorte voneinander, dauerte der abendliche Akkuwechsel ca. 2 1 1/2 Stunden, der zusätzlich erforderliche morgendliche Wech-

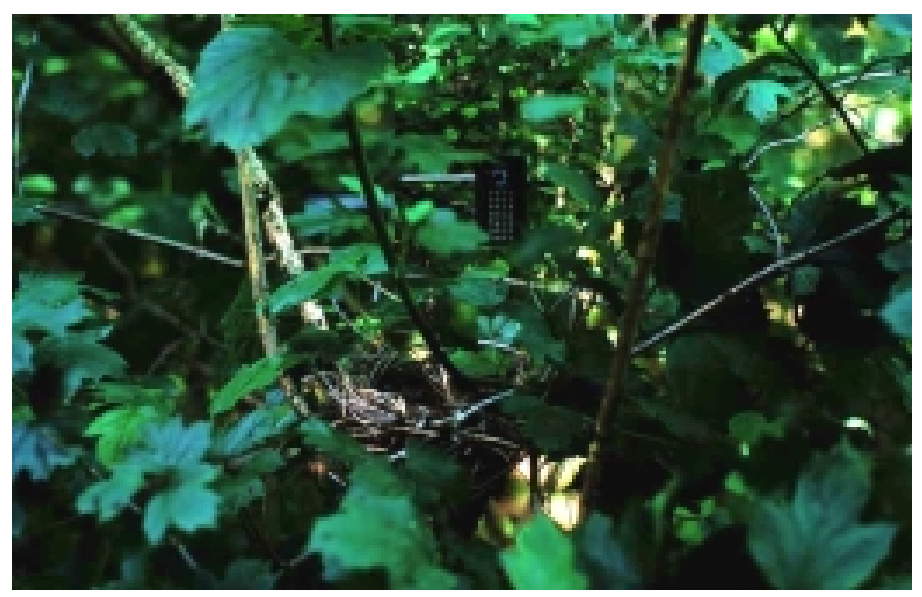

Abb. 11: LB-Kamera an einem Nest. 
sel für die fünf HR-Systeme 1 Stunde.

Insgesamt liefen die Systeme gut, obwohl verschiedentlich von Lieferantenseite darauf hingewiesen wurde, dass die Geräte für einen Betrieb im Freiland nicht ausgelegt sind. Dennoch gab es einige Unterschiede und Einschränkungen, auf die im Folgenden eingegangen werden soll.

Hauptproblem für Kameras und Rekorder in den Kisten waren Temperaturschwankungen und Feuchtigkeit. Feuchtigkeit konnte in den Kisten selbst bei sorgfältigster Abdichtung nicht ausgeschlossen werden. Spätestens wenn die Akkus bei starkem Regen gewechselt werden mussten, gelangte Feuchtigkeit in die Kisten. Wenn an unbeschatteten Stellen innerhalb der Kisten im Laufe eines Tages Temperaturdifferenzen von über $40^{\circ} \mathrm{C}$ auftraten, litten besonders die Videobänder, da sie an den gummierten Führungsrollen der Rekorder haften blieben und sich dann aufwickelten, wodurch Überwachungslücken entstanden. Dieses Problem konnte weitgehend durch den Einsatz von Silikagel vermischt mit Reis (200 g in einer löchrigen Tüte) behoben werden. Sobald sich das blaue, trockene Gel nach rot umfärbte, wurde es ausgetauscht und getrocknet. Auch im Gehäuse der HR-Kamera wurde Silikagel verwendet, da sonst die Gehäusescheibe schnell beschlagen wäre. Bei den LBGehäusen war das nicht erforderlich, weil die Geräte ausreichend Eigenwärme produzieren und so ein Beschlagen verhindern.

Weitere Probleme traten mit der Belichtung auf, die im Verlauf eines Tages erheblich schwankte. So lieferten die Platinenkameras an sehr sonnigen Tagen ein Bild, auf dem alles außer dem Nest sehr hell war und das Nest mit den Altvögeln zwar ausreichend gut, aber leicht überblendet erkennbar war. Dieses Problem trat bei den HRKameras nicht auf, da neben dem elektronischen Shutter, über den auch die LBKameras die Belichtung geringfügig steuern konnten, die Blende gut auf die Belichtung eingestellt werden konnte. So lieferten HR-Systeme auch bei Gegenlicht, das nie ganz ausgeschlossen werden konnte, scharfe und gut belichtete Bilder.

Generell waren die Bilder der HR-Kameras erheblich schärfer und kontrastreicher als die der LB-Kameras. Das lag nicht nur an der höheren Auflösung der Platine, sondern auch an der ausgezeichneten Optik. Deshalb wäre eine Interpretation von Nahrungstieren mit den LB-Systemen nicht möglich. Bei den HR-Systemen reicht die Auflösung aus, um Insektengruppen und Tiergrößen zu bestimmten.

Die Vögel schienen von den Bewegungen und Veränderungen am Nest nicht gestört. 
Vereinzelt kam es vor, dass zu Beginn einer Brutsaison Nester aufgegeben wurden, nachdem die Kamera aufgestellt wurde. Da dies jeweils vor dem Legen des dritten Eis eintrat, wurde danach gewartet bis das vierte Ei gelegt war. Aus den Aufzeichnungen geht hervor, dass Nester gelegentlich auch einige Tage nach Aufstellen der Kamera verlassen wurden, was sich nach Durchsicht der Bänder durch das Fehlen eines Altvogels erklären ließ. Die HR-Systeme erlaubten eine größere Entfernung vom Nest, dafür mussten häufiger Äste und Blätter entfernt werden. Das schien von den Vögeln zwar nicht registriert zu werden, stellte aber im Hinblick auf die Sichtbarkeit der Nester einen erhöhten Einfluss durch die Untersuchung dar.

Auch wenn die Systeme zufriedenstellend arbeiteten, sei angemerkt, dass besonders für die Rekorder durch Feuchtigkeit und Temperaturschwankungen die Grenze der Leistungsfähigkeit erreicht schien. Täglich musste mindestens eine Stunde zur Reparatur der Rekorder, Kabel, Steckverbindungen etc. eingeplant werden. Viele Defekte konnten selber gelöst werden, wie das Entfernen verklemmter Videokassetten, wozu der Videorekorder geöffnet werden musste oder Lötarbeiten an den Kabeln und Steckern. Die Rekorder wurden zusätzlich nach 1000 Betriebsstunden, d.h. etwa alle 40 Tage, zur Inspektion gebracht.

\subsubsection{Nach einem Prädationsereignis}

Nach einem Prädationsereignis, wenn also bei der Kontrolle über den Monitor und einem Blick ins Nest festgestellt wurde, dass Eier oder Jungvögel fehlten, wurden die betreffenden Videobänder überprüft. Wenn der Prädator festgestellt worden war, wurden folgende Daten aufgenommen:

- Uhrzeit des Prädationsereignisses

- Dauer des Prädationsereignisses

- Anzahl der Besuche des Prädators

- Zeit vom Flüchten der Altvögel bis zum Eintreffen des Prädators

- Zeit vom Ende der Prädation bis zur Rückkehr der Eltern

Wenn ein Nest leer war, wurden folgende Daten aufgenommen:

- Anzahl der Besuche der Eltern am Nest

- Dauer der Besuche der Eltern am Nest

- spätester Besuch der Eltern am Nest (soweit feststellbar) 


\subsubsection{Fotofallenexperimente}

Fotofallen wurden aus zwei Gründen eingesetzt: erstens um die Anzahl ermittelter Prädatoren zu erhöhen und zweitens um zu untersuchen, ob bestimmte Nester wiederholt von den gleichen Prädatoren aufgesucht wurden.

\subsubsection{Die Fotofallen}

Fotofallen, d.h. Systeme, die bei Veränderung eines externen Kontaktes eine Fotokamera auslösen, sind erhältlich, aber teuer (KUCERA \& BARRETT 1993, HERNANDEZ ET AL. 1997A, Hernandez ET AL. 1997b). Daher wurde mit Hilfe von Herrn Georg Heine, Techniker an der Universität Konstanz, ein eigenes System entwickelt (SCHAEFER \& HEINE IM DRUCK Abb. 12). Dazu wurde eine einfache AF-Kamera mit elektronischem Auslöser, integriertem Blitz, Lichtmesser, Filmvorlauf und Datenrückwand verwendet. Mit einer neu konstruierten elektronischen Schaltung wurden wesentliche Steuersysteme der Kamera ersetzt und zusätzlich der Auslöseimpuls auf die Kamera gegeben. Die Kamera wurde dazu geöffnet und die Auslösekontakte sowie der An-AusSchalter mit Hilfe eines $4^{\star}$ CMOS Schalters durch ein kleines Loch, das in den Kamerakörper gebohrt wurde, nach außen geführt. Um Kamera und externe Schaltung besser handhaben zu können, wurden sie durch einen Stecker verbunden. Ein Auslöseimpuls aktivierte zunächst den Lichtmesser und dann den Auslöser. Belichtung und Vorspulen erfolgten automatisch. Das Selbstabschalten der Kamera wurde verhindert, indem auf den An-Aus-Schalter alle $30 \mathrm{~s}$ ein Impuls gegeben wurde, der die Kamera aus- und sofort wieder einschaltete. Dadurch war die Kamera ständig im Standby-Modus. Das war besonders nachts wichtig, da sonst die Refraktärzeit zwischen einem Auslöseimpuls und dem Auslösen selbst durch das Laden des Blitzes um etliche Sekunden verzögert worden wäre. Durch diesen permanenten Standby-Modus verbrauchte der Blitz zwar kontinuierlich Strom, aber die Verzögerungszeit betrug nur 200 ms. Das gesamte System wurde durch einen Mikro-

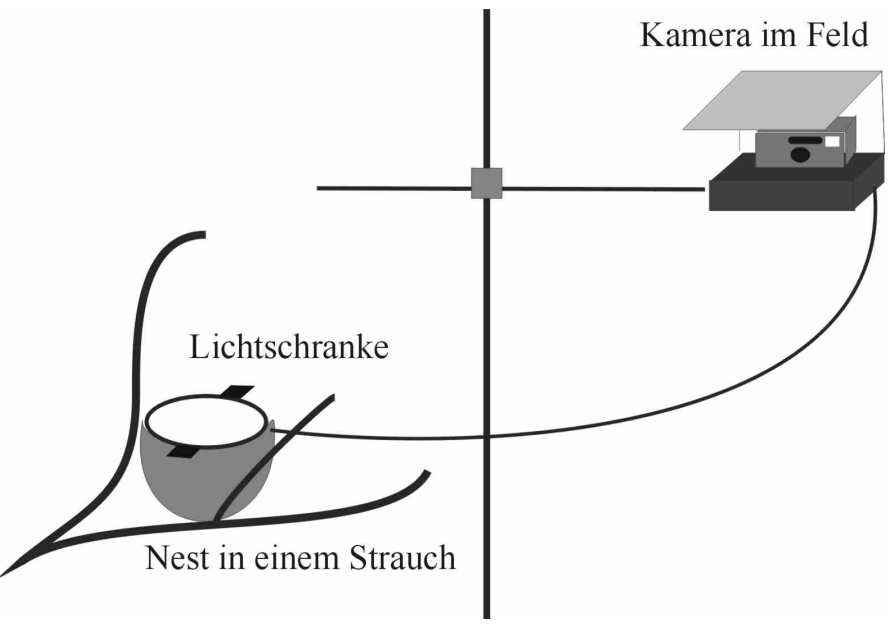

Abb. 12: Schema der Fotofalle im Feld. chip gesteuert. Dieser Chip war 
darauf programmiert, die Lichtschranke alle $5 \mathrm{~ms}$ abzufragen und die Funktionen der Kamera über den CMOS-Schalter zu steuern. Die Stromversorgung der Kameras wurde durch deren eigene Batterien gewährleistet, die etwa zwei Wochen reichten. Schaltung und IR-Lichtschranke wurden über vier 1,2 V AA-Akkus mit 1300 mAh betrieben, die je nach Außentemperatur bis zu zwei Tage halten konnten.

Die Schaltung wurde in ein wasserdichtes Kunststoffgehäuse eingebaut und die Kamera über das Stativgewinde daran befestigt. Das Gehäuse wurde an einem ca. 80 cm langen Stab befestigt, der über eine Kupplung mit einer herkömmlichen Netzstange verbunden werden konnte. Der Auslöseimpuls wurde durch eine Lichtschranke gewährleistet, die, in einen Ring integriert, auf dem Nest befestigt werden konnte. Um Temperaturschwankungen und Sonneneinstrahlung zu kompensieren, wurde die Lichtschranke durch ein moduliertes Signal von einer Phase Lock Loop (PLL)Schaltung betrieben. Die Verwendung einer Lichtschranke war für die vorgesehenen Zwecke erforderlich, auch wenn noch andere Auslösemechanismen wie Mikroschalter und Bewegungsmelder an die Schaltung angeschlossen werden könnten.

\subsubsection{Experimentelle Anordnung}

15 Fotofallen wurden konstruiert und in den Jahren 2000 und 2001 eingesetzt. Die Fallen wurden an echten Nestern aufgestellt, die ausgefressen worden waren und an solchen, bei denen der Räuber des aktiven Nests bekannt war (Abb. 13). Dazu wurde der Lichtschrankenring mit Grashalmen umwickelt (als besonders günstig haben sich leicht welke Halme der Waldhainsimse Luzula luzuloides erwiesen) und an das Nest angepasst. Die Nester wurden mit je drei unbefruchteten Eiern aus den Volieren

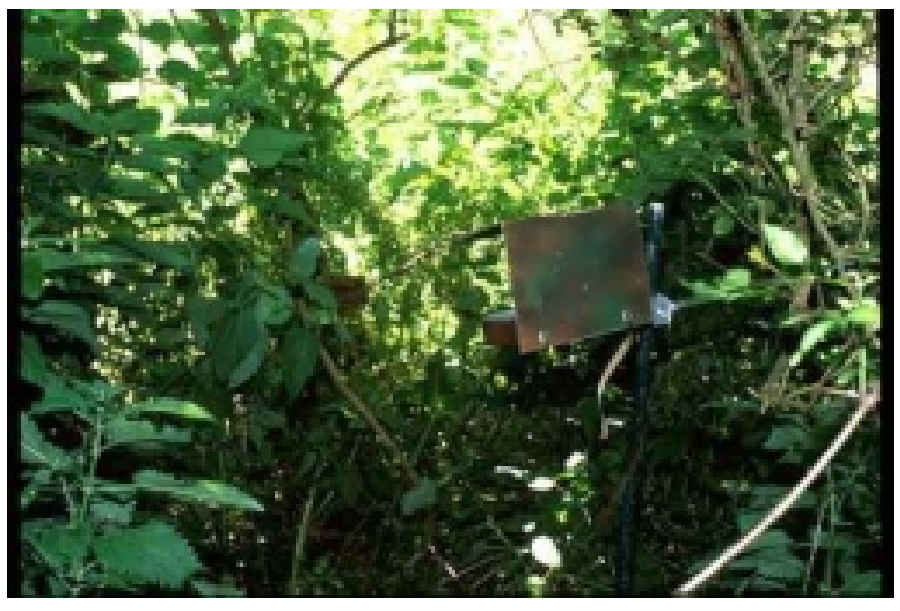

Abb. 13: Fotofalle an einem Neststandort, Sicht von hinten auf Wetterschutz. der Vogelwarte beködert. Drei Eier entsprechen schon einem Vollgelege und waren mindestens erforderlich, um die natürlichen Bedingungen wiederzugeben. Die Fotofalle wurde aktiviert und täglich mit dem Wechseln der Akkus kontrolliert. Auf diese Weise konnten Prädatoren und deren Auftreten exakt nach Datum und Uhrzeit bestimmt werden. Die 
Dauer eines Experimentes betrug 14 Tage, also etwa die Zeit, die vom Legen des dritten Eis bis zum Schlupf vergeht (BAIRLEIN 1978). Waren in einem Nest nach 14 Tagen noch alle Eier vorhanden, wurde es für 7 Tage deaktiviert, etwa die Zeit, die zwischen zwei Brutversuchen vergeht (QUERNER mündl.). Das gleiche galt für Nester, die innerhalb der 14 Tage ausgeräumt wurden. Sie wurden 7 Tage danach wieder beködert. Auf diese Weise waren Sequenzen von Prädationsereignissen am gleichen Nest möglich. Da Nester je nach Witterung während der Saison langsam zerfallen können, mussten die Fallenstandorte in einigen Fällen gewechselt werden, ohne dass eine Sequenz vervollständigt werden konnte.

\subsubsection{Erfassung von Neststandortparametern}

Zur Analyse günstiger oder weniger günstiger Standorte wurden verschiedene Neststandortparameter aufgenommen. Folgende Parameter wurden gemessen (Abb. 14):

- Nistpflanze

- Nesthöhe

- Höhe der Nistpflanze

- Breite der Nistpflanze (nur 2001)

- Entfernung des Nestes vom Rand der Nistpflanze (nur 2001)

- Sichtbarkeit des Nestes eingeteilt in fünf Klassen: <1/4, 1/4-1/2, 1/2-3/4 oder > $3 / 4$ des Nestes sichtbar und Nest nicht sichtbar, aus 6 Richtungen, von oben, unten und aus den vier Kardinalrichtungen aus je einem Meter Entfer-

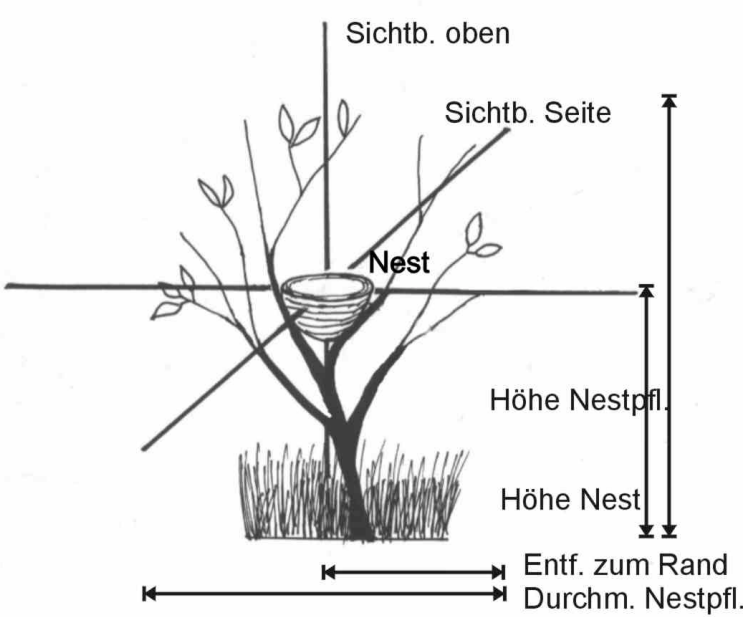
nung.

- Anzahl alternativer Neststrukturen in einem Umkreis von 20 m in drei Klassen: 1 für „einzelne“, 2 für „mehrere“, 3 für „viele Strukturen vorhanden“.

- Entfernung des Nestes vom nächsten Waldrand

- Entfernung des Nestes vom nächsten Übergang zu einer Lichtung (nur 2001).

Die Parameter waren ausgehend von möglichen Unterschieden im Suchverhalten der Nestprädatoren so gewählt, dass sie ein Nest hinsichtlich seiner Auffindbarkeit und Erreichbarkeit klassifizierten. Die ersten fünf Parameter gestatteten neben der Erreichbarkeit in der Höhe die Bestimmung der Position in der Nistpflanze. Dazu 
wurden Quotienten aus Höhe des Nestes/Höhe der Pflanze und Entfernung zum Rand der Nistpflanze/Breite der Nistpflanze gebildet. Die Bestimmung der optischen Auffindbarkeit eines Nestes ist nicht ganz einfach. Nester werden nicht immer direkt gesehen und gefunden, sondern häufig als dunkle Struktur wahrgenommen und erst beim Nachsehen als Nester identifiziert. Die Schätzung der Sichtbarkeit aus sechs Richtungen gestattet durch Bildung von Mittel- oder Min-Max-Werten verschiedene Abschätzungen der tatsächlichen Sichtbarkeit eines Nestes. Nester, die in einem Busch am Waldrand liegen, sind nach außen nicht zu sehen, von innen aus einem Meter Entfernung meist sehr gut.

Mit der Abschätzung von Alternativstrukturen sollte untersucht werden, ob die Auffindbarkeit eines Nestes davon abhängt, wie viele Strukturen in der Umgebung von einem Prädator durchsucht werden müssten. Die Einteilung war sehr grob, aber kaum anders zu treffen, da die Mönchsgrasmücke sehr variabel in der Wahl des Neststandortes ist. Ein Zählen möglicher Strukturen ist unmöglich, zumal die Entscheidungen der Vögel für oder gegen einen Standort kaum jemals nachvollziehbar sein werden (BERTHOLD mündl.). Die Werte indizieren den Strukturreichtum der Nestumgebung. Die Entfernung von $20 \mathrm{~m}$ wurde gewählt, weil in den untersuchten Waldstücken nach einem Nestverlust das Folgegelege in der Regel in weniger als 20 m Entfernung vom vorigen Nest gefunden wurde. Die Entfernung vom Rand des

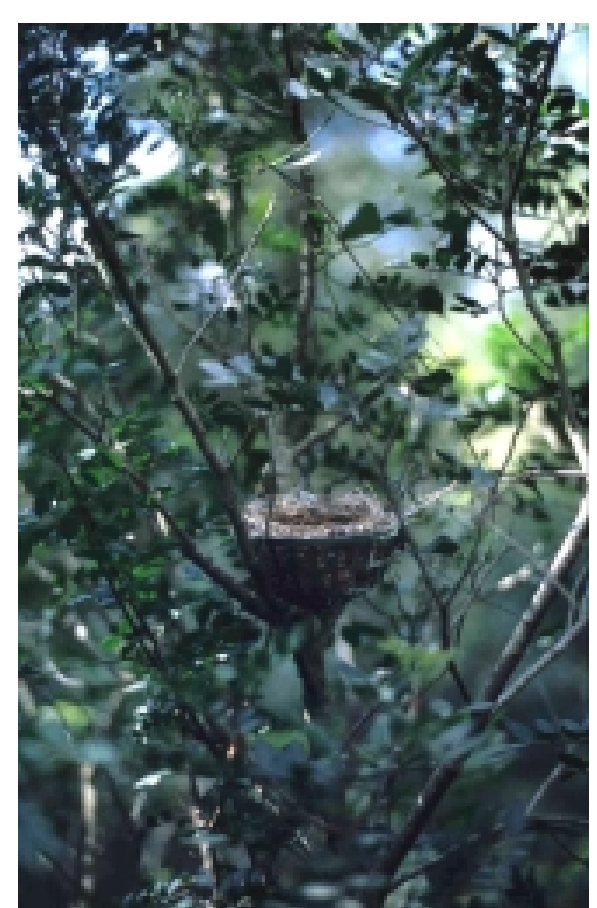

Abb. 15: Kunstnest an einem Neststandort auf Mallorca.
Waldstücks wurde gemessen, um einen möglichen Randeffekt der Prädation zu untersuchen (GATES \& GYSEL 1978).

\subsubsection{Kunstnestexperimente auf Mallorca und in Südwestdeutschland}

Zum Vergleich des Prädationsdruckes in SWDeutschland (eine Untersuchungsfläche, 1999) und auf Mallorca (beide Untersuchungsflächen, 2001) wurden Kunstnestexperimente durchgeführt. Dazu wurden korbförmige Kunststoffnesthilfen grau-grün gefärbt und mit je drei Eiern beködert an potentiellen Neststandorten ausgebracht (Abb. 15). Der Ver- 
such in Deutschland wurde mit 20 Nestern durchgeführt (Anfang Juni 1999) und einmal wiederholt (Anfang Juli 1999), auf Mallorca wurden 24 Nester eingesetzt (Ende April 2001), eine Wiederholung war aus zeitlichen Gründen nicht möglich. Die Nester wurden jeweils 10 Tage exponiert und jeden dritten Tag auf Nestraub kontrolliert. Zum Vergleich der Prädationsraten zwischen Deutschland und Mallorca wurde ein $\mathrm{X}^{2}$-Test angewendet.

\subsubsection{Siedlungsdichte der Mönchsgrasmücke auf Mallorca}

Wie viele Ergebnisse belegen, kann Nestprädation von der Siedlungsdichte der betreffenden Vogelart im Untersuchungsgebiet abhängen (KREBS 1971, DUNN 1977, MARTIN 1988). Für Südwestdeutschland liegen ausreichend Daten vor, so dass sich eigene Untersuchungen erübrigten. Um auch die Brutvogeldichte der Mönchsgrasmücke auf Mallorca einschätzen zu können, wurden nach den Vorgaben von BıBBY ET AL. (1993) in den beiden Untersuchungsflächen Revierkartierungen durchgeführt. Eine Abschätzung der Dichte aus Nestfunden (BERTHOLD 1976) war leider nicht möglich, da die Untersuchung zu Beginn der Brutsaison der Mönchsgrasmücke auf Mallorca durchgeführt wurde und noch nicht alle Paare zur Brut geschritten waren.

\subsection{Daten aus Nestfunden an der Vogelwarte 1981-2000}

Für die zahlreichen Experimente des Grasmückenprojektes (z.B. BERTHOLD 1977 BERTHOLD 1978, BAIRLEIN ET AL. 1980) an der Forschungsstelle werden seit dem Jahr 1968 mit Beginn der Brutsaison in verschiedenen Waldstücken im Raum Radolfzell Nester gesucht und Jungvögel entnommen. Die Aufzeichnungen der Daten zu dieser Nestersuche sind seit 1981 verfügbar und wurden im Rahmen dieser Dissertation digitalisiert und ausgewertet. Im Folgenden soll dargestellt werden wie die Suche erfolgte, und was in der Auswertung berücksichtigt werden musste.

\subsubsection{Untersuchungsflächen}

Als Untersuchungsflächen wurden zu Beginn der 80er Jahre bodenfrische Pappelwälder ausgewählt (Abb. 16). Dazu gehören „Böhringen“, „Espasingen I“, „Espasingen II", „Erdbeerwald“ und dazu die Flächen um das "Schloss“ in Möggingen, die eher einen Parkcharakter haben (Abb. 17). „Espasingen I“ und „Espasingen II“ wurden zu Beginn der 90er Jahre durch „Mooswald“ und „Großer Moos“ ersetzt, da absehbar war, dass durch Veränderungen in der Vegetation eine Suche nicht mehr 


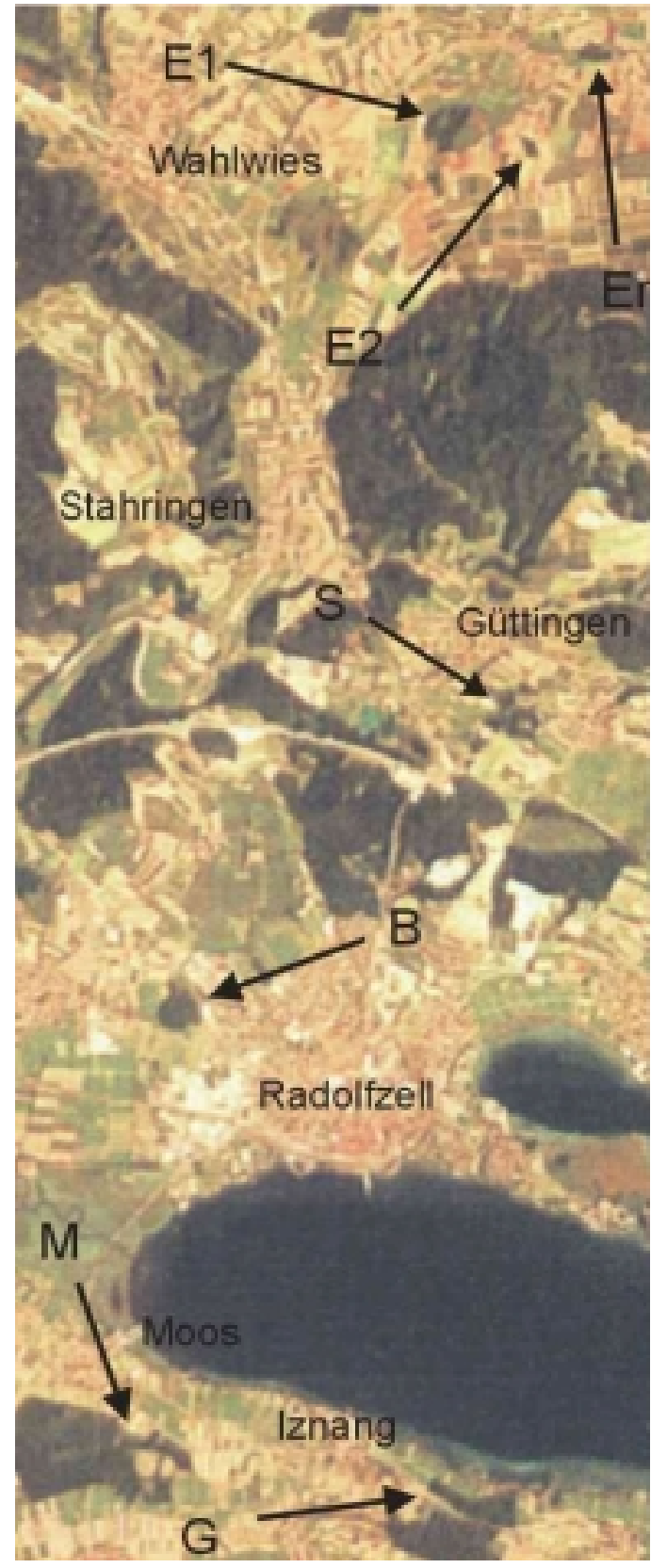

Abb. 16: Die Untersuchungsflächen für die Nestersuche an der Vogelwarte im Umkreis von Radolfzell. E1: Espasingen I, E2: Espasingen II, Er: Erdbeerwäldchen, S: Schloss Möggingen, B: Böhringen, M: Mooswald, G: Großer Moos.

Nachkontrolle ermittelt, ob das Nest verlassen war oder sich in der Legephase befand. Der Fehler bei der Bestimmung des Nestalters war hier zu vernachlässigen, da Mönchsgrasmücken in der Regel täglich ein Ei legen. Legepausen waren nach eigenen Beobachtungen sehr selten und treten vorwiegend zu Beginn der Brutsaison auf. Aus dem geschätzten Alter der Eier und Jungvögel ließen sich sowohl Legebeginn

lohnen würde. Die Mönchsgrasmückenbrutvogeldichte ist in solchen Flächen hoch, da sie optimale Voraussetzungen für Nestanlage und Jungenaufzucht bieten (BERTHOLD ET AL. 1990). Eine Suche in diesen Waldstücken kann somit sehr effizient sein. Einflussnahmen durch Beobachter sind wegen der hohen Produktivität der Lebensräume als gering einzuschätzen.

\subsubsection{Nestersuche und Aufnahme brut- biologischer Parameter}

Bei der Nestersuche bildeten 3 bis 8 Nestsucher eine Kette und durchliefen je nach Vegetationsdichte mit 5 bis $20 \mathrm{~m}$ Abstand den Wald in Bahnen. Gefundene Nester wurden in einer Skizze des Waldes notiert (Abb. 18), der Neststandort markiert. Befanden sich im Nest Jungvögel, wurde deren Alter geschätzt, was mit einem Fehler von ca. 1 Tag behaftet war, da das Wachstum der Tiere allgemein von den gegenwärtigen Ernährungsbedingungen bestimmt wird. Eier wurden geschiert (mit der Taschenlampe durchleuchtet) und die Bebrütungsdauer geschätzt. Der Fehler hierbei betrug ebenfalls einen Tag, wie aus Vergleichen des aus der Schierung resultierenden Schlupftages mit dem wirklichen Schlupfdatum ersichtlich war. Bei kalten Eiern wurde in einer 
als auch Schlupftag berechnen.

BAIRLEIN (1978) berechnet die Brutdauer vom Legetag des letzten Eis an. Die Brutdauer der Mönchsgrasmücke beträgt danach 12,1 Tage, wobei eine Abhängigkeit von der Gelegegröße besteht. Vom Legebeginn bis zum Schlupf vergeht bei 3er-, 4er-, 5er- und 6er- Gelegen nicht jeweils ein Tag mehr. Die Brutdauer ist bei größeren Gelegen offensichtlich kürzer und kompensiert die längere Legephase, wenn auch nicht vollständig (BAIRLEIN ET AL. 1980). Die errechneten Werte für den Schlupftag sind demnach nicht so genau wie der errechnete Legebeginn. Die überwiegende Anzahl der Gelege wurde im Ei-Stadium gefunden. Aus diesem Grund diente für weitere Berechnungen der Legebeginn als Referenzwert, der zurückgerechnet werden konnte. Der Schlupftermin musste meist vorausberechnet werden, eine Verifizierbarkeit des

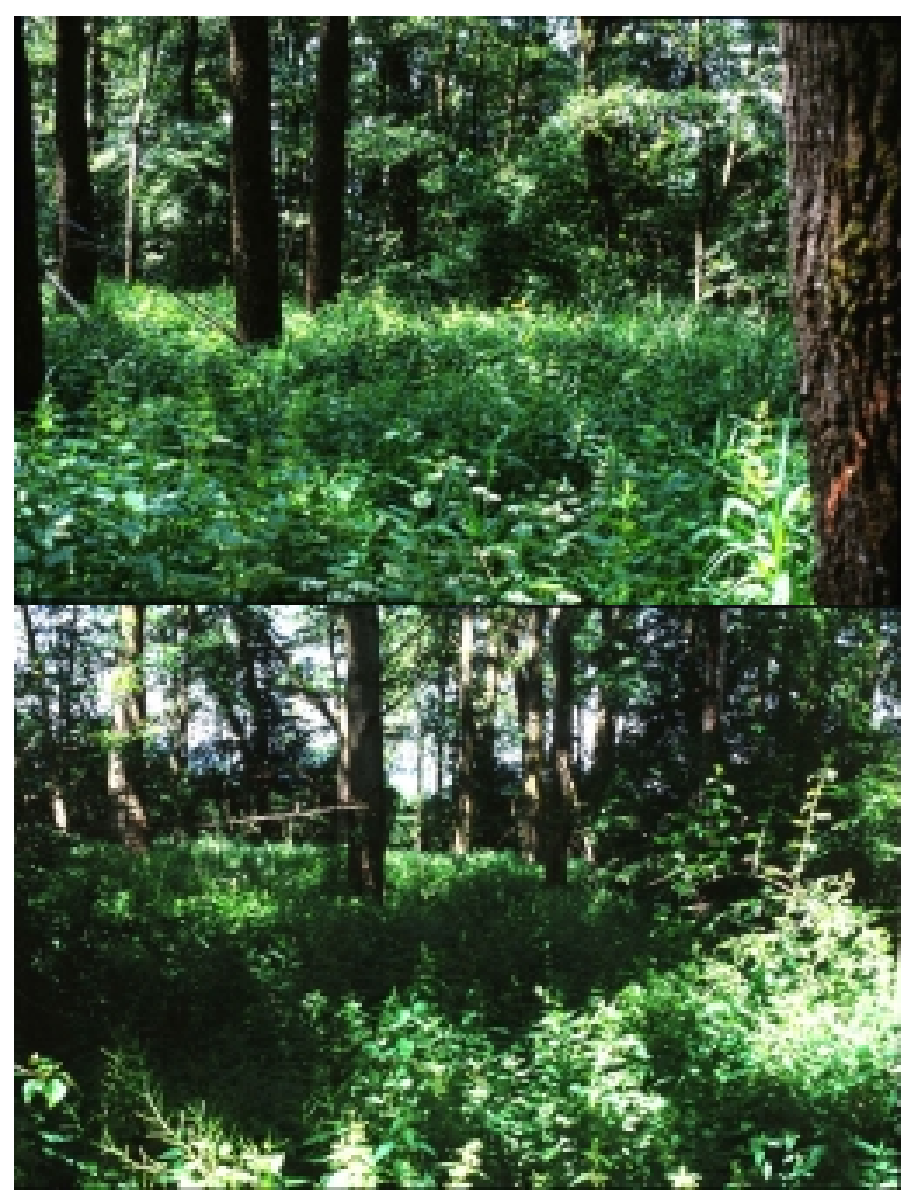

Abb. 17: Blick in zwei der Untersuchungsflächen, oben: Erdbeerwäldchen, unten: Böhringen.

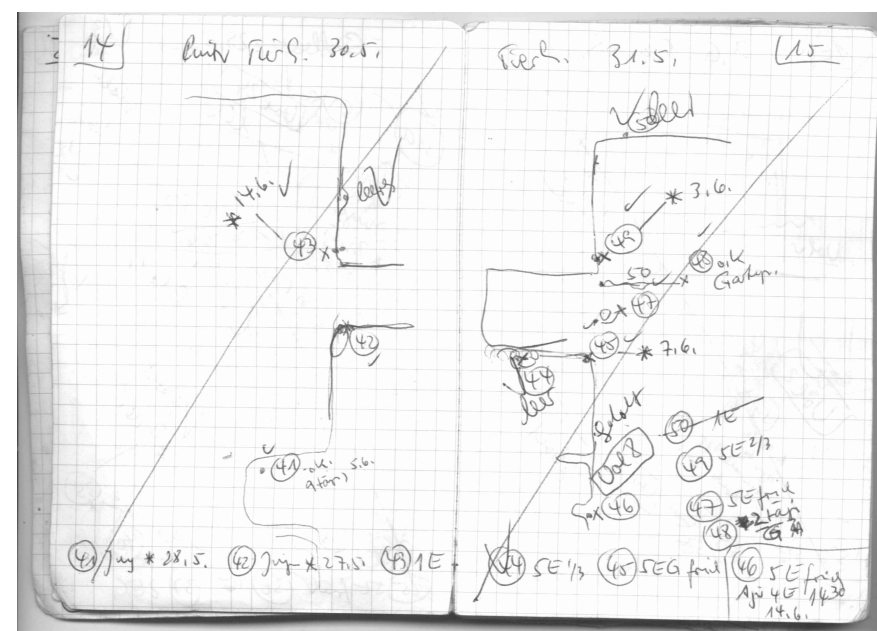

Abb. 18: Beispiel der Skizze eines Waldstücks mit den berechneten Termins war aber am betreffenden Suchtag (31.05.2000) gefundenen wegen der Verluste in der Brut- Nestern.

phase häufig nicht möglich. Es kann davon ausgegangen werden, dass sich Fehler bei der großen Anzahl der zur Verfügung stehenden Nester ausgleichen würden.

Da die Jungenaufzucht per Hand nach dem 8. oder 9. Tag nach dem Schlupf erheblich leichter fällt als davor, die Jungen nach diesem Termin, spätestens aber ab dem 
11. Tag, in der Lage sind, das Nest bei Störung zu verlassen, wurden Junge, wenn erforderlich, am 7. oder 8. Tag nach dem Schlupf aus dem Nest genommen. Die Nester wurden nach dem Fund im Allgemeinen nicht kontrolliert. Eine Kontrolle erfolgte meist um den errechneten Schlupftermin, eine weitere am 6. oder 7. Tag nach dem Schlupf, um festzustellen, ob die Jungen bereits groß genug waren, um in die Handaufzucht zu kommen. Der gleiche Wald wurde nach etwa 3 bis 4 Wochen erneut durchsucht, wenn davon ausgegangen werden konnte, dass bereits Nachgelege vorhanden waren. Für Untersuchungen zur Prädation ist dabei besonders interessant, dass aus den Kontrollen die Beobachtungszeit (exposure, MAYFIELD 1961) eines Nestes bis zur Entnahme der Jungen oder einem Prädationsereignis festgestellt werden kann. Die Ergebnisse wurden mit der Mayfield-Methode $(1961,1975)$ berechnet. Sie gestattet die Betrachtung des Bruterfolges auch von Nestern, die nicht am ersten Bebrütungstag gefunden und dann die gesamte Zeit bis zum Ende des Nestes (Ausfliegen oder Prädation) beobachtet werden konnten. Diese Methode zur Schätzung des Bruterfolgs über die Angabe einer Überlebenswahrscheinlichkeit errechnet je nach Datenlage deutlich geringere Werte als die herkömmliche Methode, bei der Prozente überlebender Nester angegeben werden (MARTIN 1993b). Seit der Veröffentlichung von MAYFIELD (1961) wurde aber verschiedentlich gezeigt, dass diese Methode verlässlichere Werte errechnet (MILlER \& JOHNSON 1978, Dow 1978, FARNSWORTH ET AL. 2000, aber: GREEN 1977). Die Methode der Nestersuche an der Vogelwarte gewährleistete es zwar, Nester sehr früh zu finden (im Schnitt 6 Tage nach Legebeginn, $s=5,7, n=2194$ ), es wurden aber auch immer wieder Nester übersehen, wie bei Nachsuchen feststellbar. Vor allem aber wurden die Kontrollen im Allgemeinen beendet, wenn die Jungen 7 oder 8 Tage alt waren und so waren durchschnittlich 10 der 28 Tage dauernden Zeitspanne vom Legebeginn bis zum Ausfliegen außerhalb der Betrachtung. Die Mayfield-Methode berücksichtigt diesen Umstand, da sie aus den vorliegenden Beobachtungsdaten tägliche Überlebenswahrscheinlichkeiten errechnet, die dann für die Dauer der Brutperiode hochgerechnet werden können. Dieser Wert ist zum Test von Unterschiedshypothesen für Fragestellungen zur Prädation erforderlich. Unterschiedshypothesen ließen sich HENSLER \& NiCHOLS 1981 folgend prüfen. Da aus Zeitgründen nicht täglich oder zweitägig Kontrollen durchgeführt wurden, wie von Mayfield (MAYFIELD 1961, MAYFIELD 1975) vorgeschlagen, war die Unsicherheit im Hinblick auf den Termin eines Nestverlustes, der zwischen zwei Kontrollen liegt, erheblich größer. Aber auch 
hier sei auf die große Anzahl der Nester verwiesen, durch die sich Fehler ausgleichen sollten, ein Umstand, auf den auch Mayfield hinweist. Anzeichen dafür, dass Nester gleich nach einer Kontrolle ausgefressen wurden und damit die Exposure beeinflussten, gibt es nicht (BERTHOLD 1977a). Von Interesse war auch, ob Jungvögel zur Zucht entnommen wurden oder das Nest insgesamt erfolgreich war. Da Nester nach dem 8. Tag nicht mehr kontrolliert wurden, die Exposure also am 8./9. Tag endet, entstand eine Unsicherheit im Hinblick darauf, ob die Jungen nicht doch in den letzten Tagen gefressen wurden. Da die tägliche Überlebenswahrscheinlichkeit i.A. über 0,93 lag und die Tiere bereits am 10. Tag bei Störung das Nest verlassen, war die Wahrscheinlichkeit, dass ein Nest in den verbleibenden ein bis zwei Tagen ausgefressen wurde, sehr gering. Für den Test von Unterschiedshypothesen war dieser Fehler unmaßgeblich, da hier nur die Exposure zur Berechnung herangezogen wurde. Für einen Vergleich der Überlebenswahrscheinlichkeiten verschiedener Nestphasen wurden diese Tage nicht berücksichtigt.

Aus den vorangegangenen Schilderungen sind die Unterschiede dieser Art der Datenerfassung zu einer Populationsuntersuchung deutlich: 1. Individuen wurden nicht markiert und ihr Schicksal war somit nicht einzeln verfolgbar, der Anteil unbekannter Tiere war nicht feststellbar; 2. der Suchaufwand richtete sich nicht nach dem Erfassungsgrad der Population, der Erfassungsgrad war nicht feststellbar und konnte allenfalls geschätzt werden; 3. Beginn und Ende der Suche in der Saison richteten sich nach den Erfordernissen der Experimente. Deswegen wurden Fragestellungen, für die eine Identität einzelner Tiere erforderlich gewesen wäre, nicht bearbeitet. Alle Auswertungen waren nest- und nicht individuenbezogen. Dass einzelne Individuen nicht bekannt waren, kann also vernachlässigt werden. Auch wenn die Gesamtzahl der Nester in einem Wald nicht feststellbar war, ließ sich der Erfassungsgrad aus Nestern, die in der Folgesuche nach drei Wochen leer oder mit fast flüggen Jungen gefunden wurden, in etwa abschätzen: er beträgt in einem Wald ca. $80 \%$. Beginn und Ende der Suche variierten von Jahr zu Jahr, ein Trend, der die Auswertung beeinflussen könnte, liegt nicht vor. Zur Analyse wurden nur Nester von Flächen herangezogen, die in jedem Jahr mit vergleichbarem Aufwand durchsucht wurden.

Folgende Daten wurden aus den Nestfunden erfasst: Fundort, Funddatum, Bebrütungsstatus / Alter der Jungvögel, Legebeginn, Schlupfdatum, Gelegegröße, Datum der vorletzten Kontrolle, Datum der letzten Kontrolle, Beobachtungszeit, Erfolg/Misserfolg, Entnommen für Experiment. 
3.2.3 Untersuchungen zum Einfluss der Landschaft auf die Prädationsrate Anzahl und unterschiedliche Größe der

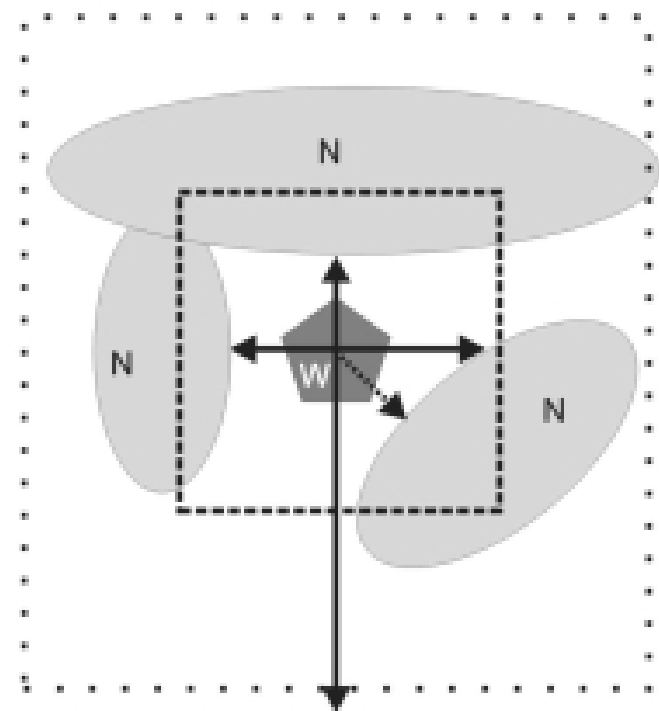

Abb. 19: Schema der für die untersuchten Waldstücke erfassten Parameter. W: Waldstück, N: Nachbarwald, durchgezogene Pfeile: Entf. in vier Richtungen, gepunkteter Pfeil: kürzeste Entf. zu einem Waldrand, gestricheltes Quadrat: Fläche mit $1 \mathrm{~km}$ und gepunktetes Quadrat: mit $2 \mathrm{~km}$ Kantenlänge.
Waldstücken erlauben es, mögliche Unterschiede in der Prädationsrate zwischen den Flächen auszuwerten und mit der Struktur einer Landschaft in Beziehung zu setzen. In Karten (TK 25) wurden folgende Parameter für die Waldsücke vermessen (Abb. 19):

- Fläche

- Randlänge

- Minimale Entfernung vom nächsten Waldstück

- Entfernung vom jeweils nächsten Waldstück in den vier Kardinalrichtungen

- Waldanteil auf einer umgebenden Fläche mit 1 km Kantenlänge (gemessen vom Mittelpunkt des Wäldchens)

- Waldanteil auf einer umgebenden Fläche von 2 km Kantenlänge (s.o.)

\subsection{Auswertung von Brutdaten aus Volieren der Vogelwarte}

Ein wichtiger Faktor für die Kompensation hoher Brutverluste ist die Fähigkeit, schnell Folgegelege zu produzieren. Leider ist diese Frage im Freiland nur mit individuell gekennzeichneten Tieren zu bearbeiten. Es hätte den Rahmen dieser Untersuchung gesprengt, wenn zu diesem Zweck eine größere Anzahl von Vögeln im Freiland gefangen, beringt und beobachtet worden wäre. Daher wurde diese Frage mit Hilfe von Volierendaten beantwortet. An der Vogelwarte wurden seit vielen Jahren Mönchsgrasmücken aufgezogen. Die Altvögel wurden ab Mitte März in die Volieren gesetzt und beginnen etwa analog zu den frei lebenden Artgenossen ab Ende April mit der Brut.

Da in Volieren die Jungenaufzucht nur gelegentlich und unter schwer wiederholbaren Bedingungen gelang, wurden die Jungen kurz nach dem Schlupf entnommen und von Hand aufgezogen. Die durchschnittliche Nestdauer betrug 11,1 $(s=2,63, n=216)$ 
Tage und nicht 15 oder 16 Tage. Das lag daran, dass besonders zu Beginn der Saison Gelege häufiger unbefruchtet waren. Die Eier wurden geschiert und unbefruchtete oder verlassene Gelege entfernt. Dadurch ist die durchschnittliche Nestdauer mit den Verhältnissen im Freiland vergleichbar. Sowohl das Entfernen aller unbefruchteten Eier als auch die Entnahme der Jungvögel in die Handaufzucht ist für die brütenden Vögel mit einem Prädationsereignis gleichzusetzen. Die Vögel reagierten wie im Freiland, indem sie Nachgelege produzierten. In Einzelfällen konnten in einer Saison bis zu 7 Brutversuche registriert werden (BERTHOLD \& QUERNER 1978). Im Allgemeinen wurde die Zahl der Brutversuche geringer gehalten, um die Tiere nicht unnatürlich zu belasten.

Jeder Legebeginn wurde beim morgendlichen Füttern erfasst, ebenso wurden die Entnahmedaten oder der Verbleib der Eier mit Datum eingetragen. Diese Daten wurden für die Jahre 1996 bis 2000 ausgewertet. Dabei wurde vermerkt, wann das erste Ei eines jeweiligen Brutversuches gelegt wurde und wann der Brutversuch, sei es durch Entnahme der Jungvögel oder durch Entfernen der Eier, endete. Das Gleiche wurde für alle weiteren Brutversuche des jeweiligen Paares notiert. 



\section{Ergebnisse}

Für die Freilandstudie wurden 233 Nester mit Eiern oder Jungen gefunden. 32 waren zum Fundzeitpunkt bereits verlassen und Informationen zu Ursachen konnten nicht nachvollzogen werden. So verblieben 201 aktive Nester, deren Schicksal verfolgt wurde. Der Auswertung der Nestfunde von Mitarbeitern der Vogelwarte von 1981-2000 (im Folgenden: Nestfunde) lagen 2315 Nester zu Grunde (Tab. 1). Es konnten in beiden Fällen nicht alle Daten für jedes Nest erfasst werden, weil einige Nester bereits ausgefressen waren, bevor das Vollgelege festgestellt werden konnte oder die Bestimmung von Neststandortparametern z.B. wegen Mahd nicht mehr sinnvoll war. Die Stichprobengrößen für die Untersuchungen wurden im Einzelnen angegeben.

Tab. 1: Anzahl der in den Untersuchungsflächen zwischen 1981 und 2000 gefundenen Nester.

\begin{tabular}{|c|c|c|c|c|c|c|c|c|}
\hline Jahr & Summe & $\begin{array}{l}\text { Böh- } \\
\text { ringen }\end{array}$ & $\begin{array}{l}\text { Erdbeer- } \\
\text { wald }\end{array}$ & $\begin{array}{c}\text { Espasinge } \\
\text { n I }\end{array}$ & $\begin{array}{c}\text { Espasinge } \\
\text { n II }\end{array}$ & $\begin{array}{l}\text { Gr. } \\
\text { Moos }\end{array}$ & $\begin{array}{c}\text { Mooswal } \\
\text { d }\end{array}$ & $\begin{array}{c}\text { Schlos } \\
\text { s }\end{array}$ \\
\hline 1981 & 115 & 26 & & 43 & 28 & & & 18 \\
\hline 1982 & 104 & 26 & 1 & 50 & 20 & & & 7 \\
\hline 1983 & 110 & 25 & & 38 & 38 & & & 9 \\
\hline 1984 & 127 & 27 & 9 & 48 & 29 & & & 14 \\
\hline 1985 & 134 & 18 & 17 & 55 & 37 & & & 7 \\
\hline 1986 & 117 & 24 & 10 & 34 & 42 & & & 7 \\
\hline 1987 & 159 & 38 & 10 & 41 & 40 & & 11 & 19 \\
\hline 1988 & 102 & 16 & 6 & 37 & 28 & & & 15 \\
\hline 1989 & 134 & 12 & 2 & 65 & 21 & 5 & 7 & 22 \\
\hline 1990 & 146 & 17 & 9 & 24 & 45 & & 20 & 31 \\
\hline 1991 & 99 & 8 & 7 & 34 & 32 & & 11 & 7 \\
\hline 1992 & 96 & 27 & 9 & 3 & & 34 & 14 & 9 \\
\hline 1993 & 150 & 48 & 8 & 3 & & 61 & 25 & 5 \\
\hline 1994 & 140 & 29 & 8 & 10 & & 36 & 28 & 29 \\
\hline 1995 & 103 & 10 & 9 & 6 & & 26 & 26 & 26 \\
\hline 1996 & 94 & 9 & 11 & 5 & & 25 & 35 & 9 \\
\hline 1997 & 121 & 25 & 9 & 15 & & 34 & 26 & 12 \\
\hline 1998 & 87 & 7 & 11 & 5 & & 33 & 30 & \\
\hline 1999 & 92 & 19 & 6 & 3 & & 22 & 41 & \\
\hline 2000 & 85 & 13 & 10 & 2 & & 22 & 38 & \\
\hline
\end{tabular}




\subsection{Prädationsmuster und Prädatoren an Nestern der Mönchs- grasmücke}

Von den 201 Nestern aus der Freilandstudie konnte für 193 Nester Daten zum Bruterfolg festgestellt werden: 88 Nester (46\%) waren erfolgreich, alle übrigen nicht. Nach den Ergebnissen der Videoüberwachungen (Tab. 6) sind 80 \% der Verluste auf Prädation zurückzuführen und ein Großteil der sonst in Frage kommenden Ursachen am Nest bestimmbar. Nester, bei denen Prädation als Verlustursache ausgeschlossen werden konnte, wurden in die folgenden Analysen nicht einbezogen. In der Freilandstudie wurde ein Nest im Schnitt 5,9 ( $s=7,0, n=193)$ Tage nach dem Legebeginn gefunden. Der Median lag am 5. Tag nach Legebeginn, die Hälfte der Nester wurde am Brutbeginn registriert, 44 Nester wurden noch vor oder mit Legebeginn gefunden. Sie wurden bis zum Ausfliegen der Jungen kontrolliert und auch danach auf Federkiele untersucht, die ein sicheres Indiz für Bruterfolg sind. Für 188 Nester konnte über mindestens eine Brutphase die Beobachtungszeit angegeben werden. Die mittlere Überlebensdauer betrug für alle Nester ab dem Legebeginn 18,2 Tage ( $s=8,34, n=180)$, für erfolglose Nester 12,9 Tage ( $s=7,36$, $\mathrm{n}=91$ ). Die meisten Nester wurden vor dem Schlupf ausgefressen. So betrug die tägliche Überlebenswahrscheinlichkeit für Nester vor dem Schlupf 0,93 bis zum Schlupf also insgesamt 0,41. Nach dem Schlupf erhöhte sich die tägliche Überlebenswahrscheinlichkeit auf 0,96 und die für die 12 Tage dauernde Nestlingsphase auf 0,59. Die Gesamtüberlebenswahrscheinlichkeit eines Nestes betrug somit 0,24. In einem Fall konnte Teilprädation festgestellt werden. In Nest 86/2000 waren die drei Jungen am 19. Juli 2000 bereits sechs Tage alt. Am darauffolgenden Tag fehlten zwei der Jungen. Das Verbliebende flog am 24.Juli aus. Die Durchsicht der Videobänder ergab einen Waldkauz als Prädator. Häufiger findet man Nester mit nur einem Ei. In diesen Fällen ließ der Prädator aus unbekannten Gründen ein Ei zurück. Die Altvögel akzeptieren nach den Videobeobachtungen den Verlust eines oder zweier Eier und fahren mit dem Brüten fort. Wenn nur noch ein Ei im Nest verblieben

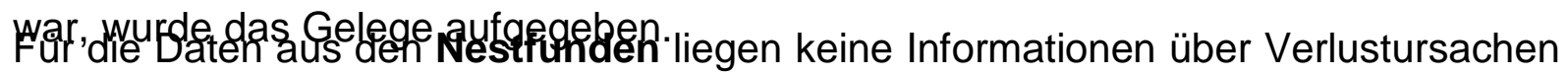
vor. Es wurde aber vermerkt, dass ein Nest „verlassen“, „ausgemäht“ oder „gekippt nach Regen“ war. In der Auswertung wurden solche Nester nicht berücksichtigt. Die Anzahl der Nester, die nicht solchen Ursachen zugeordnet werden können, ist sehr gering, wie die Videoüberwachungen zeigen. 
Aus den Nestfunden liegen für 1572 Nester Daten zum Bruterfolg vor. Für 1432 Nester konnte die Beobachtungsdauer (exposure) ermittelt werden, so dass sich die Analysen auf diese Basis stützen. Ein Nest wurde im Schnitt sechs Tage nach dem Legebeginn gefunden $(s=5,7, n$

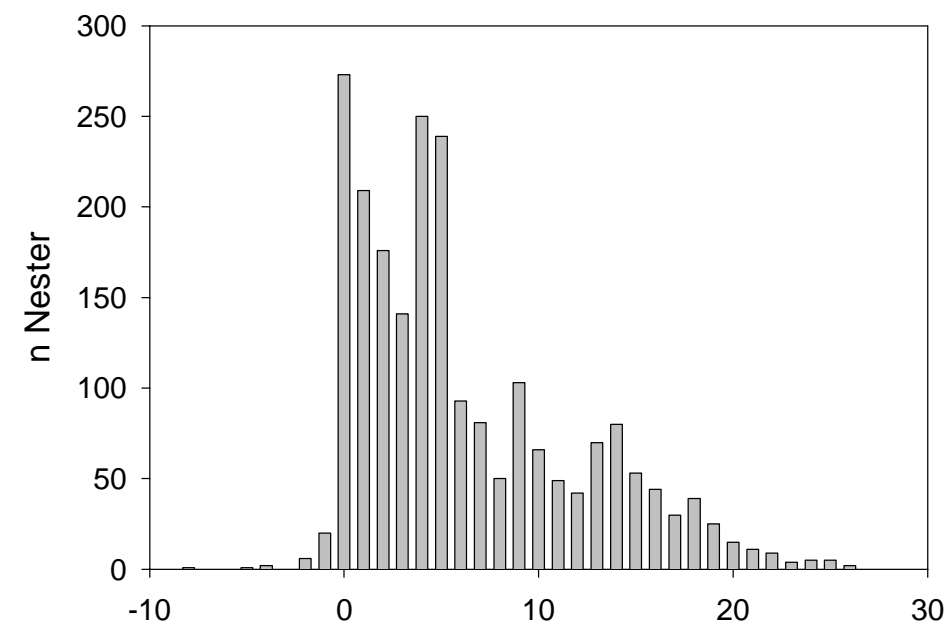
= 2194). Abb. 20 zeigt, dass der Abb. 20: Fundtag der Nester nach Legebeginn $(n=2194)$. Modalwert auf dem Legebeginn liegt, einige Nester noch davor als „im Bau“ registriert und später bei Kontrollen bestätigt wurden. Weit über die Hälfte der Nester wurde vor dem Brutbeginn gefunden.

Die durchschnittliche Überlebensdauer eines Nestes der Nestfunde betrug 14,5 Tage ( $\mathrm{s}=$

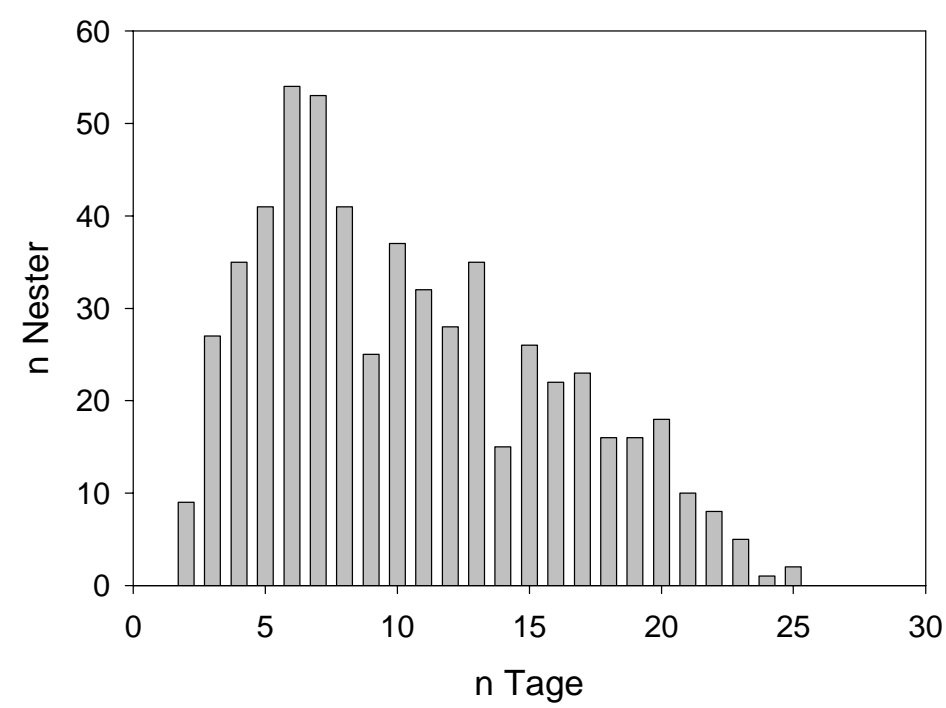
6,4, $n=1374)$. Die Nester wur- Abb. 21: Tag des Verlustes nach Legebeginn $(n=587)$. den im Mittel 10,5 ( $s=5,4, n=587$ ) Tage nach dem Legen des ersten Eis bei einer Kotrolle als „leer“ registriert. Der Median lag ebenfalls bei 10 Tagen, d.h. die Hälfte der Nester wurde in den ersten 10 Tagen nach dem Legen des ersten Eis gefunden und die Eier gefressen (Abb. 21). Daraus resultiert, dass die Überlebenswahrscheinlichkeit eines Nestes während der Bebrütungsphase mit $p=0,36$ deutlich geringer war als während der Nestlingsphase mit $p=0,64$. Nur in einem Drittel der Nester schlüpften Jungvögel; einmal geschlüpft, war die Chance auszufliegen jedoch hoch. Die hieraus resultierende Gesamtüberlebenswahrscheinlichkeit betrug $p=0,23$. Das bedeutet, dass nur ein Viertel der Nester der Freilandstudie und der Nestfunde Bruterfolg hatte und für die Mönchsgrasmücke rechnerisch vier Versuche für eine erfolgreiche Brut erforderlich waren. 


\subsubsection{Saisonale Muster der Prädation}

Eine Betrachtung saisonaler Aspekte zeigte, dass die Überlebenswahrscheinlichkeit mit dem Verlauf der Saison stieg. Die Beziehung wird am besten durch einen kubischen Zusammenhang (BORTZ 1993) dargestellt (Abb. 22). Bezogen auf die Überlebenswahrscheinlichkeit der Nester, die im jeweiligen Zeitraum ihren Legebeginn hatten, ergab sich für Nester mit Legebeginn im April 0,085 ( $n=36)$, für Nester Anfang Mai 0,17 $(\mathrm{n}=439)$ für die zweite Mai- $(\mathrm{n}=357)$ und die erste Junihälfte $(\mathrm{n}=$ 243) von etwa 0,26 , von Mitte Juni $(n=191)$ bis Mitte Juli $(n=101)$ von etwa 0,31 und für Nester mit Legebeginn in der zweiten Julihälfte von über 0,70 $(n=6)$. Dieser Zusammenhang wurde durch eine logistische Regression zwischen dem Bruterfolg und der Monatshälfte bestätigt $(n=1491, p<0,0174)$.

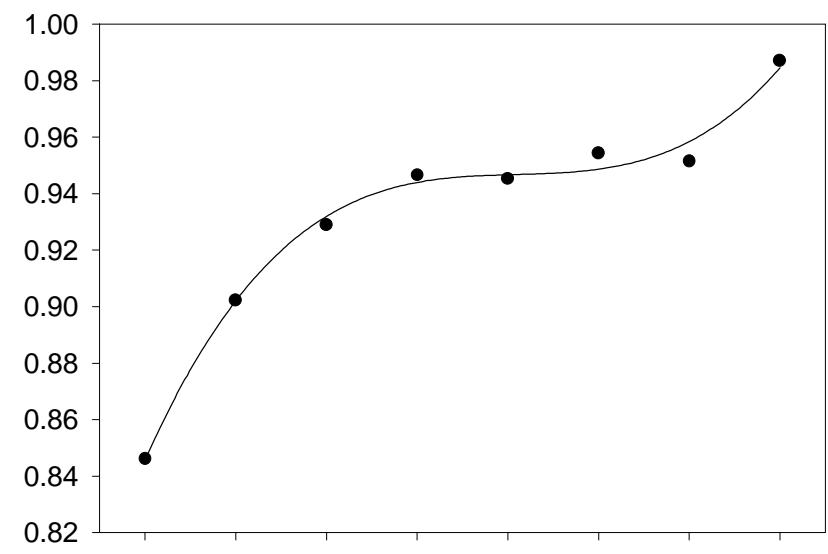

April 1 April 2 Mai 1 Mai 2 Juni 1 Juni 2 Juli 1 Juli 2

Abb. 22: Saisonale Abhängigkeit der täglichen Überlebenswahrscheinlichkeit $\left(r^{2}=0,99, n=8, p<0,0001\right)$.

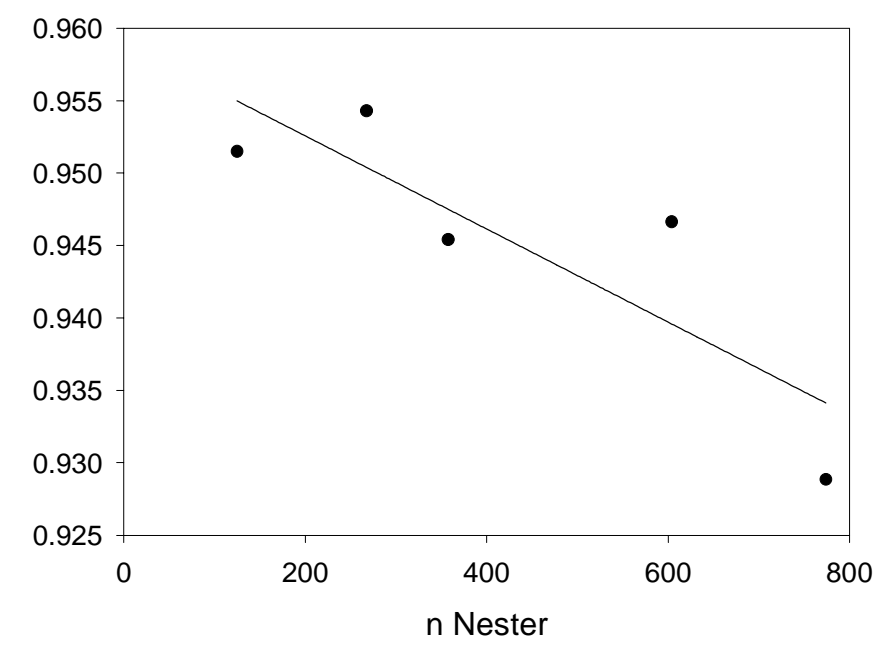

Abb. 23: Anzahl gefundener Nester in einer Monatshälfte und tägliche Überlebenswahrscheinlichkeit (n.s.).
Eine Erklärung für diese Beobachtung bietet ein Zusammenhang (n.s., $p=0,063$ ) zwischen der Anzahl in einer Monatshälfte gefundener Nester und der Überlebenswahrscheinlichkeit: je mehr Nester vorhanden sind, desto geringer ist die Überlebenswahrscheinlichkeit. Nach diesen Daten wäre die Prädation an Nestern der Mönchsgrasmücke dichteabhängig (Abb. 23).

Aus den Daten geht hervor, dass die Überlebenswahrscheinlichkeit der Nester seit 1981 zurückgegangen ist. Die Betrachtung möglicher Entwicklungen seit 1981 ergab einen linearen Zusammenhang $\left(r^{2}=0,23, n=20, p<0,03\right)$. Wurden die Waldstücke einzeln betrachtet, so ergaben sich keine Trends, die auf Veränderungen 
der Überlebenswahrscheinlichkeit der Nester seit 1981 hindeuten. Eine multiple Regression ergab, dass die Abnahme der Überlebenswahrscheinlichkeit eher auf die Wahl der Untersuchungsflächen zurückzuführen ist, aber nicht auf Unterschiede zwischen den Jahren: Wird der Fundort berücksichtigt, so ergibt sich für das Jahr kein Zusammenhang (Tab. 2).

\subsubsection{Lokale Muster der Prädation}

Die Erklärung für die Abnahme der Überlebenswahrscheinlichkeit seit 1981 liegt demnach in Unterschieden zwischen den Untersuchungsflächen. Wie dargestellt, wurden die Flächen „Espasingen I“ und „Espasingen II“ in den 90er Jahren nicht mehr systematisch durchsucht („Espasingen I“ nur einmal pro Jahr) und durch die leichter zugänglichen Waldstücke „Mooswald“ und „Großer Moos“ ersetzt (Tab. 1). Ein Vergleich lokaler Unterschiede der Überlebenswahrscheinlichkeit zeigte, dass sie in den Waldstücken nicht gleich hoch war (Tab. 3). HENSLER \& NiCHOLS 1981 stellen ein Verfahren für den Test von Unterschiedshypothesen zur Verfügung, das hier angewandt wurde (Tab. 4).

Tab. 3: Anzahl von Nestern (NN), Nestern mit Daten zum Bruterfolg (BE) und erfolgreicher Nester (EN); Summe (SB) und Mittelwert (MB) der Beobachtungstage, tägliche Überlebenswahrscheinlichkeit (tÜ) in den sieben Untersuchungsflächen der Nestersuche 1981-2000.

\begin{tabular}{|lcccccc|}
\hline Fläche & NN & BE & EN & SB & MB & tÜ \\
\hline Böhringen & 424 & 278 & 138 & 2019 & 7.8 & 0.931 \\
\hline Erdbeerwald & 152 & 112 & 67 & 1082.5 & 10.0 & 0.958 \\
\hline Espasingen I & 521 & 322 & 193 & 2422.5 & 8.4 & 0.947 \\
\hline Espasingen II & 360 & 232 & 133 & 1943 & 8.8 & 0.949 \\
\hline Gr. Moos & 298 & 229 & 91 & 1595.5 & 8.6 & 0.914 \\
\hline Mooswald & 312 & 224 & 121 & 1810 & 9.1 & 0.943 \\
\hline Schloss & 248 & 175 & 93 & 1528.5 & 9.5 & 0.946 \\
\hline
\end{tabular}


Tab. 4: Z-Werte (fett), Differenzen und summierte Standardabweichung (kursiv) der Berechnungen nach Hensler \& Nichols (1981) für Unterschiede der täglichen Überlebenswahrscheinlichkeit zwischen den Untersuchungsflächen. Z-Werte über 1,65 zeigen Unterschiede auf einem Signifikanzniveau von 0,05 .

\begin{tabular}{|c|c|c|c|c|c|c|c|}
\hline Fläche & $\begin{array}{l}\text { Böh- } \\
\text { rin. }\end{array}$ & Erdbeer & Espas. I & $\begin{array}{l}\text { Espas. } \\
\text { II }\end{array}$ & $\begin{array}{l}\text { Gr. } \\
\text { Moos }\end{array}$ & Moosw. & Schloss \\
\hline Böhringen & & $3,4^{*}$ & 2,29 * & 2,63 * & 2,02 * & $1,77^{*}$ & 2,29 * \\
\hline Erdbeerwald & 0,027 & & $1,67^{*}$ & 1,16 & 5,16 * & 1,96 * & 1,51 \\
\hline Espasingen I & 0,016 & 0,011 & & 0,38 & 4,34 * & 0,55 & 0,055 \\
\hline Espasingen II & 0,018 & 0,009 & 0,002 & & $4,44^{*}$ & 0,84 & 0,39 \\
\hline Großer Moos & 0,017 & 0,045 & 0,033 & 0,036 & & 3,61 * & 3,88 * \\
\hline Mooswald & 0,012 & 0,015 & 0,004 & 0,006 & 0,03 & & 0,47 \\
\hline Schloss & 0,016 & 0,012 & 0,0004 & 0,003 & 0,033 & 0,003 & \\
\hline
\end{tabular}

Tab. 5: Fläche (F), Randlänge $(\mathrm{R})$ und Rand/Fläche-Index (R/F), mittlere (EW) und minimale (MW) Entfernung zum nächsten Wald und Waldanteile auf $1 \mathrm{~km}^{2}$ (W 1) und $4 \mathrm{~km}^{2}$ (W 4) Umgebung der sieben Untersuchungsflächen.

\begin{tabular}{|lccccccr|}
\hline Ort & $\mathbf{F}(\mathbf{h a})$ & $\mathbf{R}(\mathbf{k m})$ & $\mathbf{R} / \mathbf{F}$ & $\mathbf{E W}(\mathbf{k m})$ & $\mathbf{M W}(\mathbf{k m})$ & $\mathbf{W} 1$ & $\mathbf{W} 4$ \\
\hline Böhringen & 9,3 & 1,36 & 0,15 & 3,3 & 0,7 & 9,3 & 2,8 \\
\hline Erdbeerwald & 2,6 & 0,82 & 0,32 & 1,4 & 0,6 & 2,6 & 12,5 \\
\hline Espasingen I & 7,7 & 1,27 & 0,17 & 2,8 & 0,4 & 9,6 & 2,9 \\
\hline Espasingen II & 1,8 & 0,58 & 0,32 & 2,7 & 0,6 & 1,9 & 3,6 \\
\hline Großer Moos & 12,0 & 2,30 & 0,18 & 4,8 & 0 & 34,1 & 18,5 \\
\hline Mooswald & 6,3 & 2,13 & 0,34 & 3,9 & 0 & 29,4 & 19,5 \\
\hline Schloss & 6,5 & 0,93 & 0,14 & 0,6 & 0 & 12,2 & 18,6 \\
\hline
\end{tabular}

Danach war die Überlebenswahrscheinlichkeit in "Böhringen" und "Großer Moos" geringer als in allen anderen. Weitere Unterschiede bestanden zwischen „Erdbeerwald“, „Espasingen I“ und „Mooswald“: Im „Erdbeerwald“ war die Überlebenswahrscheinlichkeit höher. Die verbliebenen Kombinationen ergaben keine Unterschiede, einen Trend zeigte die Differenz zwischen „Erdbeerwald“ und „Schloss“ ( $p<0,065)$. Um darzustellen, inwieweit Unterschiede zwischen den Flächen und deren Einbet- 
tung in die Landschaft für Unterschiede der Überlebenswahrscheinlichkeit der Nester ursächlich sind, wurde dieser Faktor mit den gemessenen Parametern (Tab. 5) in Beziehung gesetzt. Die Ergebnisse zeigten, dass eine signifikante statistische Abhängigkeit zwischen der Flächengröße und der Prädationsrate bestand. Die Überlebenswahrscheinlichkeit sank mit zunehmender Größe der Fläche (Abb. 24).

Die anderen Parameter ergaben keine solche Zusammenhänge. Allerdings zeigte sich für die Randlänge ein Trend: Je länger der Waldrand einer Fläche, desto geringer die Überlebenswahrscheinlichkeit der Nester (n.s.). Dieser Faktor ist stark mit der Flächengröße korreliert. In einer logistischen Regression wurde deswegen der Einfluss der Fläche und der Residuen aus der Korrelation

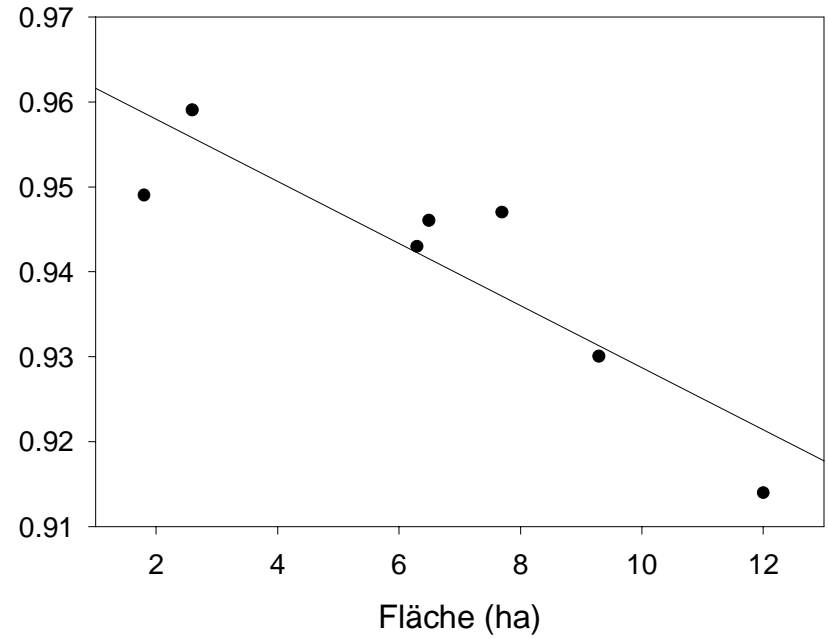

Abb. 24: Zusammenhang zwischen der täglichen Überlebenswahrscheinlichkeit und der Flächengröße $\left(r^{2}=0,79, n\right.$ $=7, p=0,0078)$.

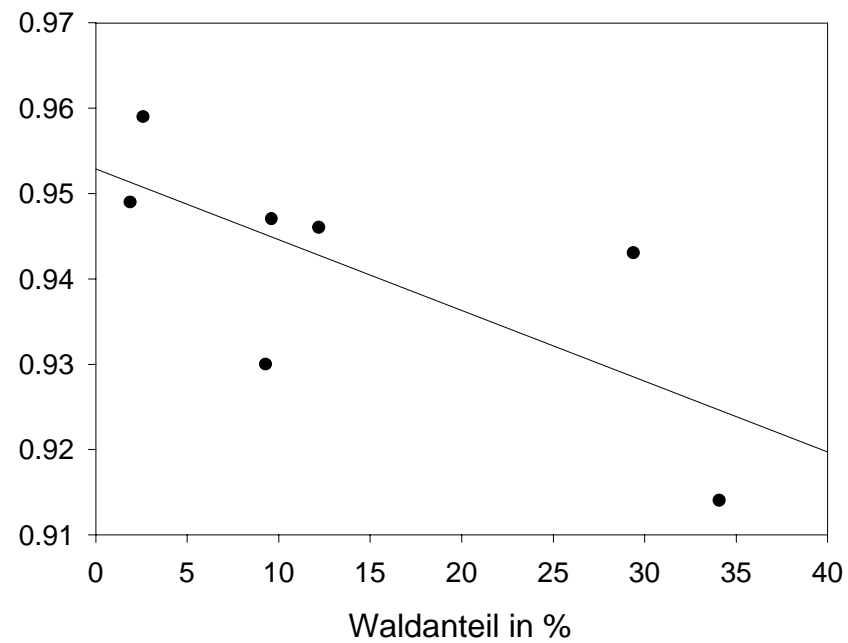

Abb. 25: Zusammenhang zwischen der täglichen Überlebenswahrscheinlickeit und dem Waldanteil auf einer umgebenden Fläche von $1 \mathrm{~km}$ Kantenlänge $\left(\mathrm{r}^{2}=0,51, \mathrm{n}=7\right.$, $\mathrm{p}=0,074$, n.s.). zwischen der Randlänge und Fläche auf den Bruterfolg untersucht: Danach gibt es keinen Einfluss der Randlänge auf den Bruterfolg ( $n=1572, p=0,49)$, wenn die Flächengröße mit einbezogen wird, die einen sig-nifikanten Einfluss hat $(p<0,0001)$. Die Analyse der Quotienten aus Rand/Areal der Untersuchungsflächen teilte die Nester in den Waldstücken in zwei Gruppen: die eine mit einem Index von 0,14 - 0,18 (viel Fläche pro Rand), die andere zwischen 0,32 und 0,34 (wenig Fläche pro Rand, Tab. 5). Eine Abhängigkeit der Überlebenswahrscheinlichkeit von diesem Parameter gibt es nicht.

Die Analyse des in der Freilandstudie erfassten Parameters „Entfernung zum Wald- 
rand" ergab ebensowenig einen Unterschied zwischen prädierten und ausgeflogenen Nestern ( $t$-Test, $p=0,80, n=120$ ) wie der Parameter „Entfernung zum nächsten Übergang" (t-Test, $p=0,73, n=137$ ). Ein Randeffekt der Prädation liegt nach diesen Daten und den dargestellten Ergebnissen der Nestfunde nicht vor.

Die Einbettung der Waldstücke in die Landschaft zeigte einen Trend, der auf einen Effekt des Anteils an Waldfläche in einem umgebenden Quadrat von $1 \mathrm{~km}$ Seitenlänge auf die Überlebenswahrscheinlichkeit hinweist: Je höher der Waldanteil in der näheren Umgebung, desto geringer die Überlebenswahrscheinlichkeit (n.s., Abb. 25). Für ein Quadrat mit $2 \mathrm{~km}$ Seitenlänge ergab sich keine Beziehung $\left(r^{2}=0,04, n=7, p\right.$ $<0,67)$. Die Auswertung der Entfernungen in vier Richtungen ergab einen Trend zwischen dem Mittelwert der gemessenen Entfernungen und der Überlebenswahrscheinlichkeit auf der Untersuchungsfläche. Je weiter eine Fläche im Mittel von den benachbarten entfernt ist, desto geringer ist die Überlebenswahrscheinlichkeit (n.s.). Die minimale Entfernung zum Rand des nächstgelegenen Waldes ergab keine Abhängigkeit bezüglich der Überlebenswahrscheinlichkeit ( $p=0,25$, n.s.).

\subsubsection{Prädatoren}

\subsubsection{Videoüberwachungen}

Tab. 6: Verlustursachen von Nestern der Mönchsgrasmücke nach den Videoüberwachungen.

\begin{tabular}{|lccr|}
\hline Verlustursache & $\mathbf{N}$ & \% Verluste & \% Gesamt \\
\hline Prädation & 45 & 76,3 & 35,7 \\
\hline Wetter & 3 & 5,1 & 2,4 \\
\hline Verhungern & 1 & 1,7 & 0,8 \\
\hline Verlassen & 3 & 5,1 & 2,4 \\
\hline Fallen aus Nest & 1 & 1,7 & 0,8 \\
\hline Überbrüten & 1 & 1,7 & 0,8 \\
\hline Störungen & 5 & 8,5 & 4,0 \\
\hline Summe & $\mathbf{5 9}$ & $\mathbf{1 0 0 , 0}$ & $\mathbf{4 6 , 8}$ \\
\hline
\end{tabular}

Zwischen 1999 und 2001 wurden an 132 Nestern Videokameras aufgebaut. Zu Beginn jeder Saison wurden einige $\mathrm{Ne}$ ster vermutlich durch die Installation der Kamera in Nestnähe verlassen, so dass sich die Anzahl wirklich überwachter $\mathrm{Ne}$ ster auf 126 belief, von denen 67 erfolgreich waren: 45 Nester wurden von Prädatoren ausgefressen, das sind 76,3 $\%$ der Verluste. 14 fielen anderen Ursachen zum Opfer (Tab. 6): In einem Fall wurden die Eier im Nest von Hagel zerschlagen. In zwei weiteren Fällen wurde nach sehr starkem Regen das Nest so beschädigt, dass die Eier in den folgen- 
den Tagen aus dem Nest fielen. In einem Nest verhungerten 1999 die Jungen, die während einer Regenperiode geschlüpft waren. Drei $\mathrm{Ne}$ ster wurden verlassen, ohne dass ein Zusammenhang mit äußeren Faktoren ersichtlich war. Die Banddurchsichten ergaben weiterhin, dass in einem Fall über zwei Tage kein Männchen und in den beiden anderen Fällen kein Weibchen am Nest erschien. In einem weiteren Fall fielen die Jungvögel ohne ersichtliche Ursache (Unerfahrenheit der Altvögel?) nacheinander aus dem Nest. Das Zerstören der Eier durch die Altvögel in einem weiteren Nest ist unter Umständen damit zu erklären, dass die Eier taub und deswegen überbrütet waren. Fünf weitere Nester sind durch folgende Störungen verlorengegangen: Eines war nah an einem Feldweg angelegt und wurde durch einen vorbeifahrenden Trecker so stark geschüttelt, dass die Eier hinausgeschleudert wurden. Das wiederholte sich an

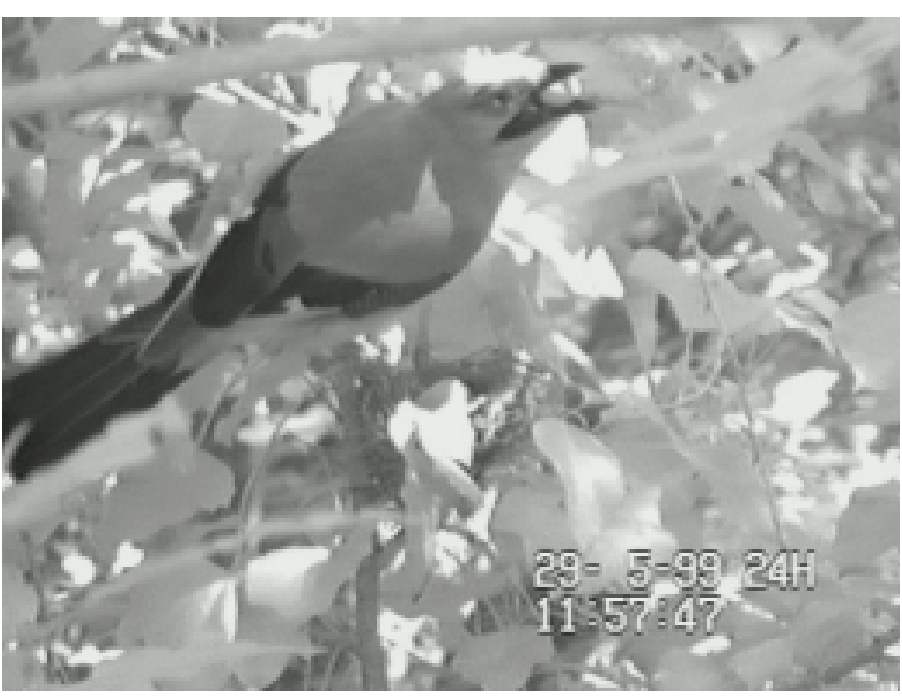

Abb. 26: Eichelhäher entnimmt ein Ei aus einem Nest (4/1999).

Tab. 7: Prädatoren und deren jeweilige Anteile an Prädationen, Verlusten und insgesamtem Ergebnis aller Nester.

\begin{tabular}{|lcccr|}
\hline Prädator & $\mathbf{n}$ & \% Präd. \% Verluste & \% Gesamt \\
\hline $\begin{array}{l}\text { Eichelhäher } \\
\begin{array}{l}\text { Garrulus } \\
\text { glandarius }\end{array}\end{array}$ & 24 & 60,0 & 45,8 & 21,4 \\
\hline $\begin{array}{l}\text { Aaskrähe } \\
\text { Corvus corone }\end{array}$ & 2 & 5,0 & 3,8 & 1,8 \\
\hline $\begin{array}{l}\text { Waldkauz } \\
\text { Strix aluco }\end{array}$ & 1 & 2,5 & 1,9 & 0,9 \\
$\begin{array}{l}\text { Steinmarder } \\
\text { Martes foina }\end{array}$ & 6 & 15,0 & 11,4 & 5,4 \\
$\begin{array}{l}\text { Mauswiesel } \\
\text { Mustela nivalis }\end{array}$ & 1 & 2,5 & 1,9 & 0,9 \\
\hline $\begin{array}{l}\text { Fuchs } \\
\text { Vulpes vulpes }\end{array}$ & 3 & 7,5 & 5,7 & 2,7 \\
\hline $\begin{array}{l}\text { Wildschwein } \\
\text { Sus scrofa }\end{array}$ & 1 & 2,5 & 1,9 & 0,9 \\
\hline $\begin{array}{l}\text { Maus } \\
\text { Apodemus } \\
\text { spec. }\end{array}$ & 2 & 5,0 & 3,8 & 1,8 \\
\hline \begin{tabular}{l} 
Summe \\
\hline
\end{tabular} & 40 & 100 & $\mathbf{7 6 , 3}$ & $\mathbf{3 5 , 7}$ \\
\hline
\end{tabular}
zwei weiteren Nestern, als in der Nacht zum 15.5.99 bzw. 18.5.00 flüchtendes Wild durch den Nestbusch brach. Die anderen beiden Nester wurden durch „Waldrandpflege“ freigelegt und danach verlassen.

Insgesamt wurden 53 Prädationsereignisse an 40 Nestern aufgezeichnet.. Diese höhere Zahl ergab sich, weil Eichelhäher ein Nest häufig mehrmals besuchten, um Eier oder Junge zu holen: An 24 verschiedenen Nestern konnten 35 Ereignisse gefilmt 


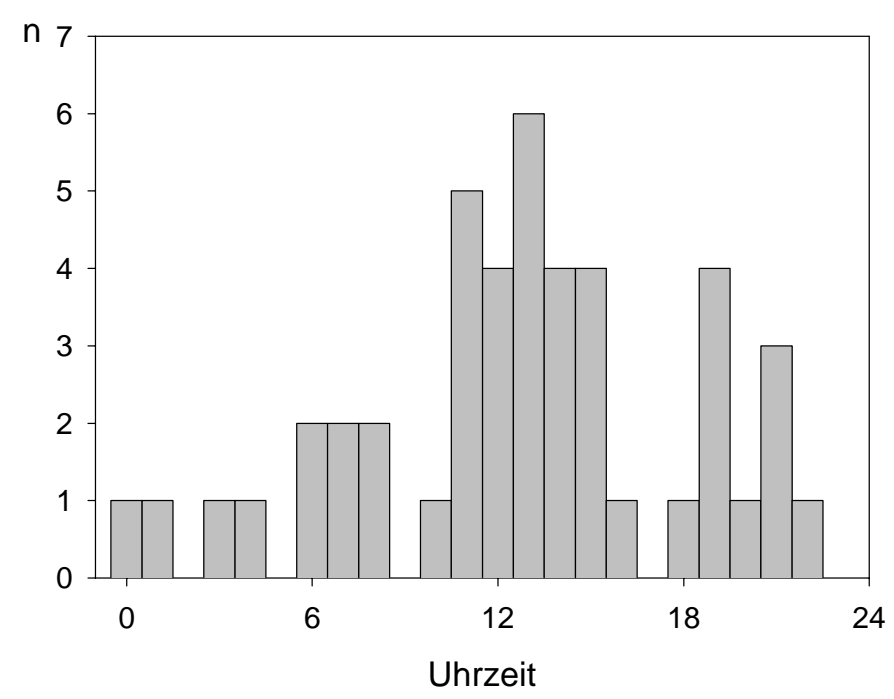

Abb. 27: Uhrzeit der Prädationsereignisse $(n=44)$.

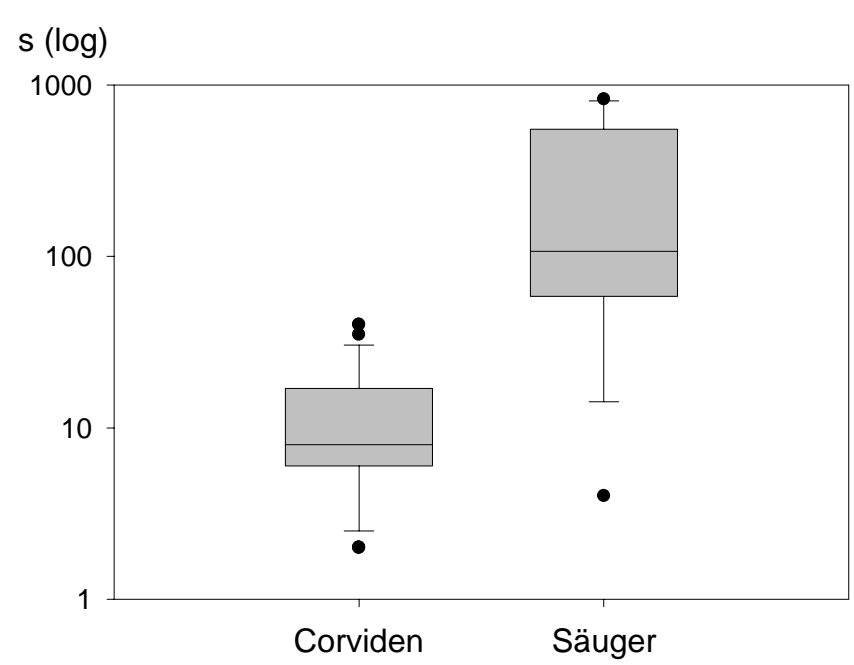

Abb. 28: Aufenthaltsdauer der Prädatoren am Nest (t-Test, $\mathrm{p}<0,0001, \mathrm{n}=30$ ). werden. Eichelhäher (Abb. 26) waren somit für $60 \%$ der Nestprädationen und $46 \%$ der Gesamtnestverluste bei der Mönchsgrasmücke verantwortlich. Die übrigen 18 gefilmten Prädationsereignisse verteilten sich auf sieben verschiedene Säugetiere und Vögel (Tab. 7). An 5 prädierten Nestern konnten wegen technischer Probleme die Räuber nicht eindeutig bestimmt werden.

Abgesehen von diesen Besuchern am Nest gab es verschiedene andere Tiere, die nicht zum Verlust der Brut führten. Kohlmeisen und Zaunkönige wurden mehrfach festgestellt, nachdem ein Nest ausgeräumt war. Haselmäuse und Waldmäuse wurden vielfach nachts in Nestnähe beobachtet, ohne dass die Brut beeinträchtigt wurde.

Nach den vorliegenden Beobachtungen konnte ein Nest zu jeder Tageszeit aufgesucht werden (Abb. 27). Allerdings kamen verschiedene Nesträuber zu unterschiedlichen Uhrzeiten: Eichelhäher und Krähen kamen bei Tageslicht. Der früheste Besuch eines Eichelhähers war um 6:39 Uhr, der späteste um 20:35 Uhr. Alle anderen Arten erschienen nachts und in der Dämmerung zwischen 21:27 (Steinmarder) und 7:41 Uhr (Mauswiesel). Aufgrund der Dominanz der Corviden insgesamt war die Verlustwahrscheinlichkeit nachts wesentlich geringer. Nach Einbruch der Nacht, also von etwa 22:00 Uhr bis in die frühen Morgenstunden, gingen selten Nester verloren, die meisten um die Mittagszeit und am Abend (Abb. 27).

Die Dauer der Aufenthalte schwankte sehr stark. Eichelhäher benötigten nur wenige 
- im Minimum 2 - Sekunden, um Eier oder Jungvögel zu holen. Abgesehen vom Mauswiesel und Krähen blieben die anderen Arten länger am Nest, die Mäuse jeweils etwa eine Viertelstunde, die Steinmarder bis zu 3 1/2 Minuten. Fasst man Corviden und Säuger jeweils zusammen, so ergibt sich ein signifikanter Unterschied in der Aufenthalts-dauer am Nest (Abb. 28).

Aus Spuren am Nest lässt sich der Prädator nicht zweifelsfrei bestimmen, wie die Videoaufnahmen zeiTab. 8: Verlustursachen und Spuren am Nest $(\mathrm{n}=80)$.

\begin{tabular}{|c|c|c|c|}
\hline Ursache & $\begin{array}{l}\text { Keine } \\
\text { Spuren }\end{array}$ & Nest hängt & Schalen \\
\hline Eichelhäher & 22 & 1 & \\
\hline Fuchs & & 3 & \\
\hline Steinmarder & 1 & 4 & \\
\hline Krähe & 2 & & \\
\hline Maus & 2 & & \\
\hline Waldkauz & 1 & & \\
\hline Mauswiesel & 1 & & \\
\hline Wildschwein & & 1 & \\
\hline Hagel & & & 1 \\
\hline Regen & & 2 & \\
\hline Störung & 2 & 2 & 2 \\
\hline Nicht gefilmt & 24 & 7 & 2 \\
\hline Summe & 55 & 20 & 5 \\
\hline
\end{tabular}

gen. Eichelhäher haben in keinem Fall Spuren am Nest hinterlassen, ebensowenig Krähen, Steinmarder, Waldkauz und Mauswiesel. Wenn Jungvögel im Nest waren, war auch bei den Mäusen keine Spur zu sehen, größere Tiere zerstörten das Nest häufig. Dazu kommen andere Verlustursachen: So lagen nach einem Hagelschlag leere Eierschalen in Nest, die Regenfälle führten zu hängenden Nestern und in den Fällen, in denen das Nest durchgeschüttelt wurde, blieb ein leeres, aber unbeschädigtes Nest, evtl. noch mit Eierschalen zurück (Tab. 8).

Das Verhalten der Prädatoren am Nest ist sehr unterschiedlich. Neben der Dauer der Aufenthalte schwankte vor allem die Anzahl der Besuche am Nest. Die meisten Prädatoren kamen nur einmal zum Nest. In den meisten Fällen wurden dann alle Eier und Jungvögel geholt. Beim Eichelhäher hingegen scheint das die Ausnahme zu sein. Nur in einem Fall fraß der Eichelhäher auf einmal das ganze Gelege. In allen anderen Fällen brauchte er dazu zwei bis fünf Besuche. Bei zwei Nestern wurde, obwohl das Nest leer war, ein Eichelhäher aufgezeichnet. Jungvögel wurden immer einzeln geholt, Eier wurden direkt am Nest verschluckt, aber häufig nur eins oder zwei. Wenn man davon ausgeht, dass es sich jeweils um den gleichen Vogel handelte, erschien dieser Eichelhäher

Tab. 9: Anzahl der Besuche der Eichelhäher am Nest und der Tage, über die sich die Besuche erstreckten.

\begin{tabular}{|lrrrrr|}
\hline Anzahl: & $\mathbf{1}$ & $\mathbf{2}$ & $\mathbf{3}$ & $\mathbf{4}$ & $\mathbf{5}$ \\
\hline Besuche & 1 & 8 & 3 & 4 & 1 \\
\hline Tage & 14 & 2 & 1 & - & - \\
\hline
\end{tabular}
an drei aufeinander folgenden Tagen (Tab. 9). 


\subsubsection{Fotofallen}

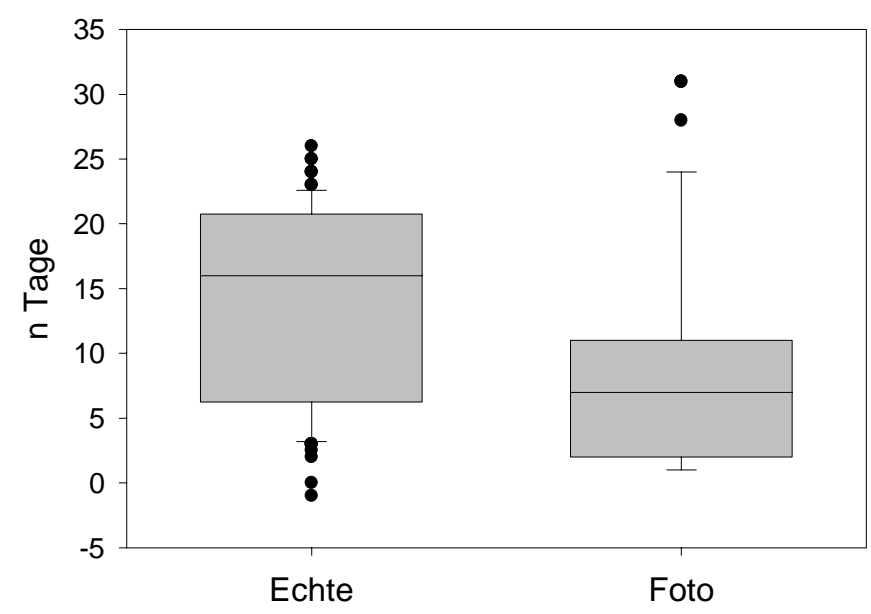

Abb. 29: Überlebensdauer ab Legebeginn in Tagen von Video- und Fotofallennestern (t-Test, $\mathrm{p}<0,0026, \mathrm{n}=$ 116).

Fotofallen wurden in den Jahren 2000 und 2001 an insgesamt 36 verschiedenen prädierten Nestern aufgestellt, dabei wurden 66 Setups durchgeführt. An 20 Nestern wurde nur einmal ein Fotosetup aufgebaut, weil zwei Wochen darauf die Saison zu Ende war oder das Nest durch Regen etc. zerstört war. An sechs Nestern konnte der Versuch zweimal wiederholt werden, an sieben Nestern dreimal und an drei Nestern viermal. Aus 37 Nestern wurden die Eier entfernt oder zerstört. Die übrigen 29 Nester waren nach zwei Wochen „erfolgreich“. Die durchschnittliche Überlebensdauer der nicht erfolgreichen Nester betrug 9,5 Tage ( $=8,8, n=37$ ), der Median lag bei 7 Tagen. Die Überlebensdauer der ausgeräumten Fotofallennester war also kürzer als die natürlicher Nester (Abb. 29).

Mit dem Fotofallenexperiment sollte die Brutphase möglichst gut simuliert werden, weshalb die Dauer eines Setups auf 14 Tage begrenzt wurde. In einigen Fällen wurde diese Zeit verlängert, um zu sehen, wie lange es dauern würde, bis alle Nester gefunden sind. Hierbei zeigte sich, dass ein Nest bis zu 31 Tage ungestört bleiben konnte und dann erst die Eier gefressen wurden. Einige Nester waren auch nach 4 1/2 Wochen nicht entdeckt.

Tab. 10: Anteile einzelner Prädatoren an Nestern mit (alle Ereignisse) und ohne (einfach) Doppelbeobachtungen an einem Nest und der Erstprädatoren an einem Nest (Anzahl Beobachtungen).

\begin{tabular}{|llll|}
\hline & Eichelhäher & Nager & Singvögel \\
\hline Alle Ereignisse & $20 \%(19)$ & $64 \%(60)$ & $16 \%(15)$ \\
\hline Einfach & $33 \%(15)$ & $43 \%(20)$ & $24 \%(11)$ \\
\hline Erstprädatoren & $45 \%(13)$ & $45 \%(13)$ & $10 \%(3)$ \\
\hline
\end{tabular}

Insgesamt wurden 230 Fotos von Prädatoren an den Nestern gemacht. Da in vielen Fällen mehrere Fotos vom gleichen Ereignis aufgezeichnet wurden, konnten sie 94 einzelnen Prädationsereignissen zugeordnet werden (Tab. 10). 
Davon waren 19 Eichelhäher und 15 andere Singvögel wie Kohlmeise, Heckenbraunelle, Zaunkönig und Mönchsgrasmücke. Die übrigen 60 Ereignisse gingen auf das Konto von Nagern (Abb. 30): 35 entfielen auf Waldmäuse und 11 auf Haselmäuse Muscardinus avallanarius. Einmal konnte eine Wühlmaus Microtus spec. und fünfmal eine Rötelmaus Clethrionomys spec. identifiziert werden. An einem Nest trat zweimal ein Siebenschläfer Glis glis auf und einmal wurde ein Eichhörnchen Sciurus vulgaris fotografiert. In sechs Fällen war die artgenaue Bestimmung eines Nagers nicht möglich. Größere Säuger als Eichhörnchen sind an den Fotofallen nicht aufgetreten. Die Ergebnisse sind damit deutlich verschieden von denen der Videoüberwachungen. Während Mäuse und Nager insge- Tab. 11: Saisonalität der Prädatoren an Fotofallen mit samt an den echten Nestern nur allen Beobachtungen ( $\mathrm{a}, \mathrm{p}<0,025)$ und unter Ausausnahmsweise auftraten, waren schluss von Mehrfachbeobachtungen $(e, p<0,005)$ am sie an den Fotofallen die häufig- gleichen Nest.

sten Prädatoren. Betrachtet man nicht alle aufgetretenen Prädatoren, sondern geht davon aus, dass am selben Nest an verschiedenen Tagen oder zu unterschiedlichen Uhrzeiten aufgetretene Mäuse und

\begin{tabular}{|c|c|c|c|}
\hline & Eichelhäher & Nager & Singvögel \\
\hline Vor 20.6. e & $48 \%(12)$ & $44 \%(11)$ & $8 \% \quad(2)$ \\
\hline Nach 20.6. e & $14 \% \quad$ (3) & $43 \% \quad(9)$ & $43 \% \quad(9)$ \\
\hline Vor 20.6. a & $28 \%(15)$ & $48 \%(26)$ & $24 \%(13)$ \\
\hline Nach 20.6. a & $8 \% \quad(4)$ & $68 \%$ (34) & $24 \%(12)$ \\
\hline
\end{tabular}

Eichelhäher Territoriumshalter und somit identisch waren, verschiebt sich dieses Bild nur wenig. Nager machten dann nicht mehr $64 \%$ der Prädatoren aus, sondern nur noch 43 \%. Bezieht man sich ausschließlich auf Erstprädationen, steigt der Mäuseanteil auf 45 \%. Nach diesen Daten waren Mäuse auch dann die Hauptprädatoren, wenn Dopplungen ausgeschlossen werden (Tab. 10).

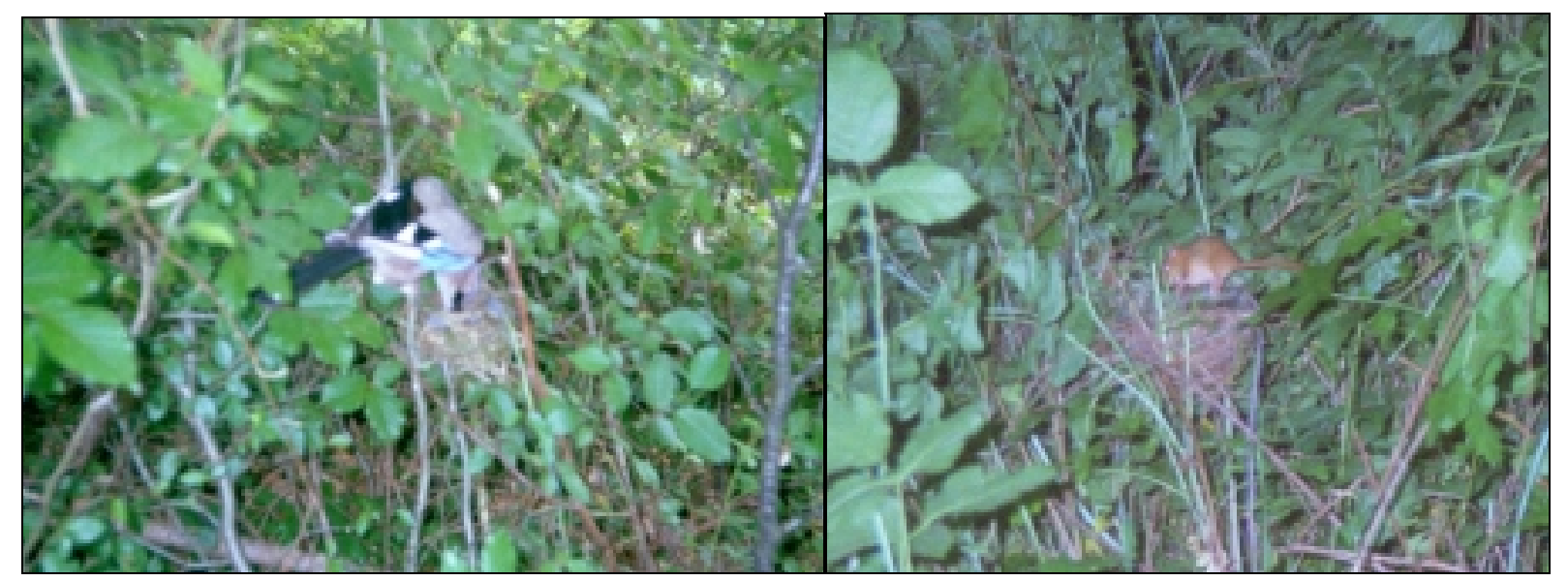

Abb. 30: Eichelhäher und Haselmaus an einer Fotofalle. 
Die Ergebnisse zeigen einen saisonalen Effekt der Prädatoren (Tab. 11). Vor Mitte Juni traten signifikant mehr Eichelhäher an den Nestern auf als danach $\left(X^{2}\right.$-Test, $p<$ $0,025)$. Dieser Unterschied wird deutlicher, wenn man Dopplungen ausschließt $\left(X^{2}-\right.$ Test, $p<0,005)$. Haselmäuse traten ausschließlich vor dem 20.6. auf, Waldmäuse in dieser Zeit dagegen nur vereinzelt.

In den Fällen, in denen Eichelhäher Eier geholt haben, waren keine Spuren am Nest zu finden. Dagegen hinterließen Echtmäuse und Haselmäuse stets leere Eierschalen. Die Wühlmausartigen entfernten keine Eier und hinterließen auch keine Spuren. Offensichtlich waren sie nicht in der Lage, die Eier zu öffnen. Kohlmeisen schienen die Eier aufzupicken und den Inhalt zu fressen. Bei den übrigen Singvögeln konnte das nicht eindeutig belegt werden, da sie jeweils am gleichen Tag wie Mäuse auftraten und deswegen eine eindeutige Zuordnung der Spuren am Nest nicht möglich war.

Die zeitliche Einteilung der Prädationsereignisse zeigte, dass Nager ausschließlich von der Abenddämmerung ab etwa 18:30 Uhr bis zum Morgengrauen um etwa 6:00 Uhr auftraten. Vögel traten ab 6:00 Uhr bis etwa 20:30 Uhr auf. In der Abenddämmerung waren also alle in Frage kommenden Prädatoren an den Nestern aktiv. Abb. 31 zeigt wegen der Dominanz der Nager einen deutlichen Unterschied zu den Ergebnissen der Videoüberwachung (Abb. 27).

Durch das wiederholte Aufstellen der Fotofallen gelangen 11 Bestimmungen des Erstprädators bei aufeinanderfolgenden Setups. Fünfmal traten Eichelhäher am gleichen Nest bei aufeinanderfolgenden Setups als Erstprädatoren auf. Viermal folgte

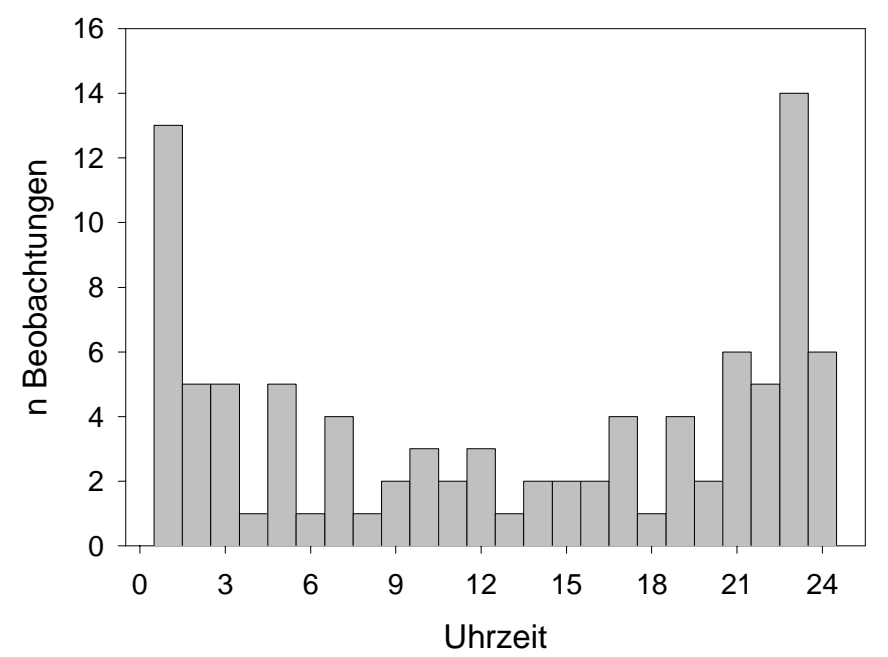
auf einen Eichelhäher ein Nager (Waldmaus) und einmal ein Eichelhäher auf einen Nager. In zwei Fällen konnten drei aufeinanderfolgende Prädatoren bestimmt werden. Im ersten Fall folgten auf eine Haselmaus zwei Eichelhäher, im zweiten auf zwei Eichelhäher eine Waldmaus.

Abb. 31: Uhrzeit der Prädationen an Fotofallen $(n=94)$. 


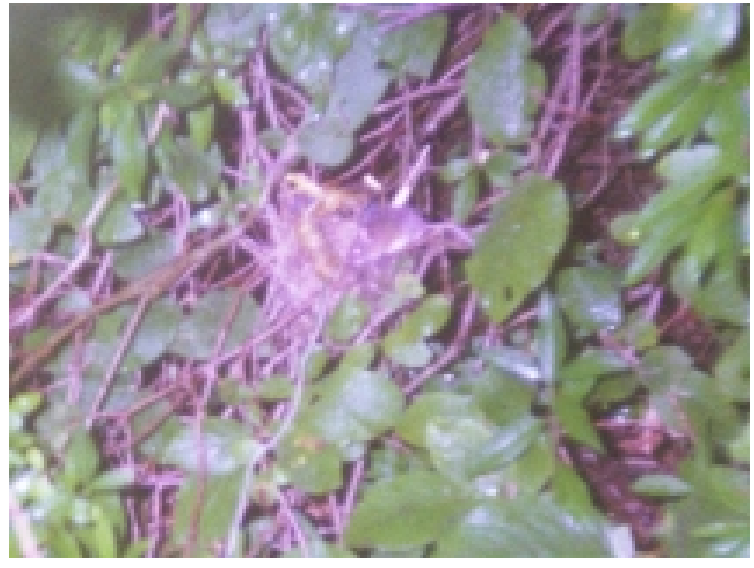

Abb. 32: Waldmaus am Nest 84/2000.

Betrachtet man die Anzahl jeweils verschiedener Prädatoren innerhalb eines Setups, zeigt sich, dass in den meisten Fällen (67\%) nur ein Prädator auftrat, der
Tab. 13: Übereinstimmungen von Prädatoren an aktiven Nestern (V) und Fotofallen (F 1-5, E: Eichelhäher. S: Steinmarder, F: Fuchs, H: Haselmaus, N: Nager; K: Kohlmeise).

\begin{tabular}{|lllllll|}
\hline Nest & V & F 1 & F 2 & F 3 & F 4 & F 5 \\
\hline $11 / 2000$ & E & H & & & & \\
& & & & & & \\
\hline $12 / 2000$ & E & H & E & H & E & N \\
\hline $21 / 2000$ & E & E & E & & & \\
\hline $38 / 2000$ & S & $H$ & $E$ & & & \\
& & & & & & \\
\hline $73 / 2000$ & $E$ & $N$ & & & & \\
\hline $5 / 2001$ & $F$ & $K$ & & & & \\
\hline
\end{tabular}
dann bis zu 8-mal zum Nest gekommen wäre. In 6 Fällen wurden 2 verschiedene Prädatoren fotografiert, in 2 Fällen 3 Arten und in 1 Fall sogar 5 an einem Nest, wobei fraglich ist, ob es sich ausschließlich um Prädatoren handelte (Singvögel, s.o.). Es zeigte sich, dass im Verlaufe einer Saison bis zu 7 verschiedene Prädatorenarten zu Fotos an den Fallen führten. Eine Analyse aller auftretenden Paarungen innerhalb eines Setups zeigt, dass Waldmäuse die Nester bis zu 8-mal an bis zu 4 aufeinander folgenden Tagen und zu ähnlichen Uhrzeiten besuchten. Als Beispiel sei hier Nest 84/2000 dargestellt (Tab. 12, Abb. 32).

An 6 Nestern deren Prädatoren durch Videoaufzeichungen bestimmt worden waren, konnten mit Fotofallen Folgeprädatoren bestimmt werden (Tab. 13). Durch die Mehrfachaufstellungen an den Fotofallen ergaben sich 11 VideoFoto-Kombinationen. In 4 Fällen (36 \%) stimmte der Folge-

Tab. 12: Datum und Uhrzeit der Besuche von Waldmäusen am Nest 84/2000.

\begin{tabular}{|ll|}
\hline Datum & Uhrzeit \\
\hline 08.07 .2000 & $21: 19$ \\
\hline 08.07 .2000 & $22: 51$ \\
\hline 09.07 .2000 & $01: 15$ \\
\hline 09.07 .2000 & $01: 39$ \\
\hline 09.07 .2000 & $21: 09$ \\
\hline 10.07 .2000 & $20: 39$ \\
\hline 10.07 .2000 & $21: 04$ \\
\hline 11.07 .2000 & $21: 27$ \\
\hline
\end{tabular}
prädator mit dem tatsächlichen überein, in $64 \%$ war an der Fotofalle ein anderer als am aktiven Nest. 


\subsection{Reduktion der Prädationsrate}

\subsubsection{Verhalten der Altvögel}

Es konnte nicht beobachtet werden, dass ein Altvogel versucht hat, das Nest gegen einen eintreffenden Prädator zu verteidigen. Entweder wurden die Altvögel überrascht und verließen das Nest abrupt, was nicht immer zu gelingen schien, wie ein Fund von Federn eines Altvogels in einem zerstörten Nest zeigt, oder sie verließen das Nest unmittelbar vor dem Eintreffen des Prädators. Der Median für 18 protokollierte Fälle lag bei 2 Sekunden, die Quartile bei 0 bzw. 16 Sekunden. Bei fütternden Vögeln war unter Umständen kein Altvogel anwesend. Wenn ein Altvogel auf dem Nest saß, schien er den Eichelhäher zu beobachten, wie dieser näher kam. Er duckte sich ins Nest, so dass man von der Seite lediglich seinen Schnabel sah und erst kurz bevor ein Eichelhäher am Nest eintraf glitt er vom Nest. Das gleiche Verhalten zeigten Vögel bei der Nesterkontrolle. Bei solchen Kontrollen konnte mehrfach beobachtet werden, wie die Altvögel versuchten, den Beobachter zu verleiten, indem sie eine Verletzung vortäuschten. Es wurde aber auch beobachtet, dass Vögel, nachdem sie zunächst geflohen waren, zum Nest zurückkamen und versuchten den Beobachter zu vertreiben. Dieses Verhalten wurde einmal auch auf den Videobändern dokumentiert: Nachdem eine Maus bereits vier Minuten am Nest verbracht hatte, kam das Weibchen zurück und flog mehrere, erfolglose Attacken.

Tagsüber waren die Altvögel kurze Zeit nach einer Prädation wieder am Nest. Sowohl die Weibchen (Mittel: 13:39 min, $n=18$ ) als auch die Männchen (Mittel = 12:35 min, $n=11$ ) kamen nach einer Viertelstunde zurück. Es gab Fälle, in denen einer der Partner erst nach Stunden wieder auf den Videobändern erschien. Zwischen Tag und Nacht bestand ein Unterschied ( $t$-Test, $p<0,05, n=18$ ), denn nachts brauchten die Weibchen etwa eine Stunde, um wieder zum Nest zu kommen, für Aussagen über das Verhalten der Männchen lagen diesbezüglich keine ausreichenden Daten vor.

Spätestens nach einem Totalverlust verließen die Altvögeln das Nest. Im Allgemeinen wurden die Nestkameras nach einem Prädationsereignis abgebaut. In einigen Fällen ergab sich ein weiterer Tag der Überwachung oder der Prädator kam früh am Tag, so dass festgestellt werden konnte, wieviel Zeit von der Prädation bis zum letzten registrierten Besuch eines Altvogels verging und wieviele Besuche es gab. 
Es lagen acht Beobachtungen für Weibchen und sechs für Männchen vor. Für Weibchen lag der Mittelwert bei 3 h 11min, die Spanne lag zwischen einigen Minuten und fast 9 Stunden. Bei Männchen vergingen im Schnitt nur etwa 2 1/2 Stunden, die Spanne war aber ähnlich groß wie bei den Weibchen (Abb. 33). Auch in der Anzahl der Besuche zeigten sich Unterschiede zwischen Männchen und Weibchen. Während Männchen nur noch zweimal (Median, $\mathrm{n}=6$ ) zum Nest kamen, erschienen Weibchen gut fünfmal (Median $=5,5, \mathrm{n}=8$, Abb. 34). Statistische Unterschiede lassen sich mit den vorliegenden Daten nicht darstellen.

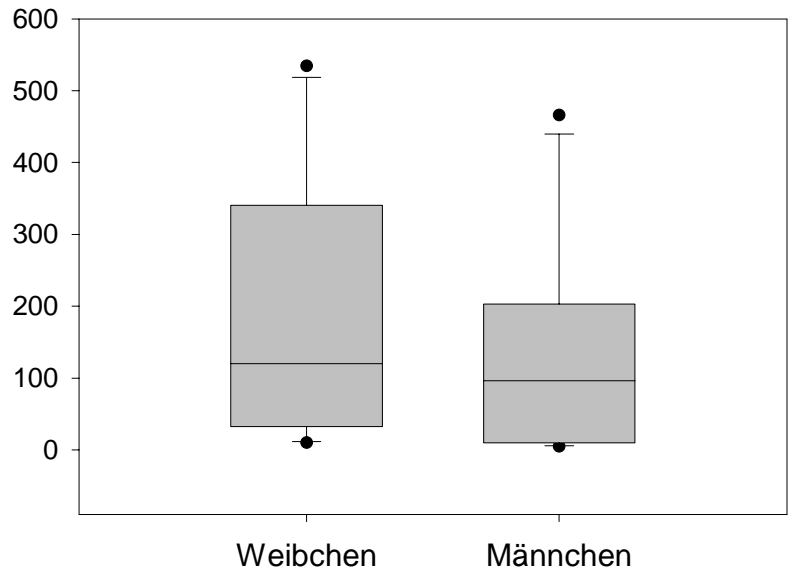

Abb. 33: Dauer bis zum letzten Besuch eines Altvogels nach Prädation (Weibchen: $n=8$, Männchen: $n=6$, n.s.).

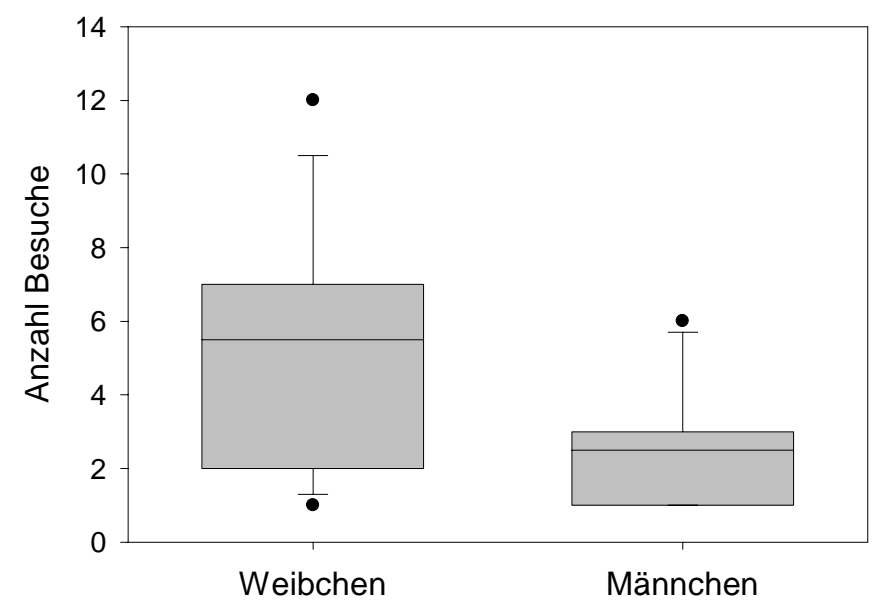

Abb. 34: Anzahl der Besuche nach Prädation (Weibchen n $=8$, Männchen $\mathrm{n}=6$, n.s.). 


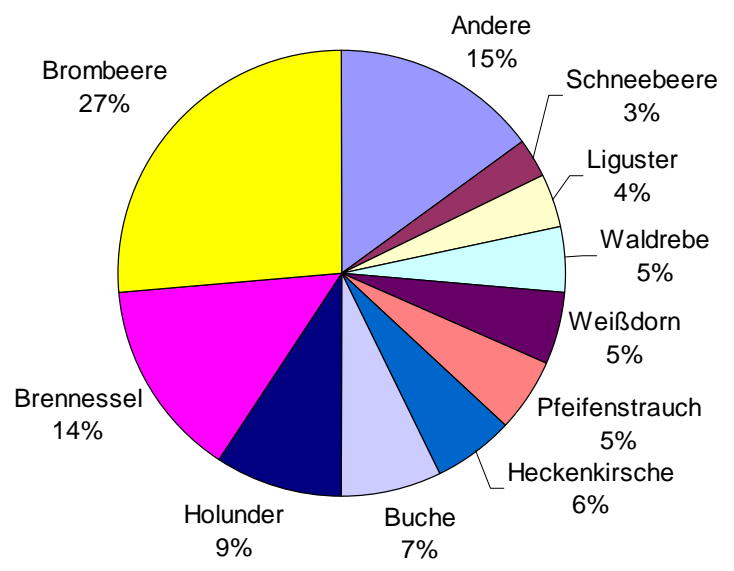

Abb. 35: Nistpflanzen der Mönchsgrasmücke in der Umgebung von Radolfzell aus Daten der Freilandstudie ( $\mathrm{n}=$ 152).

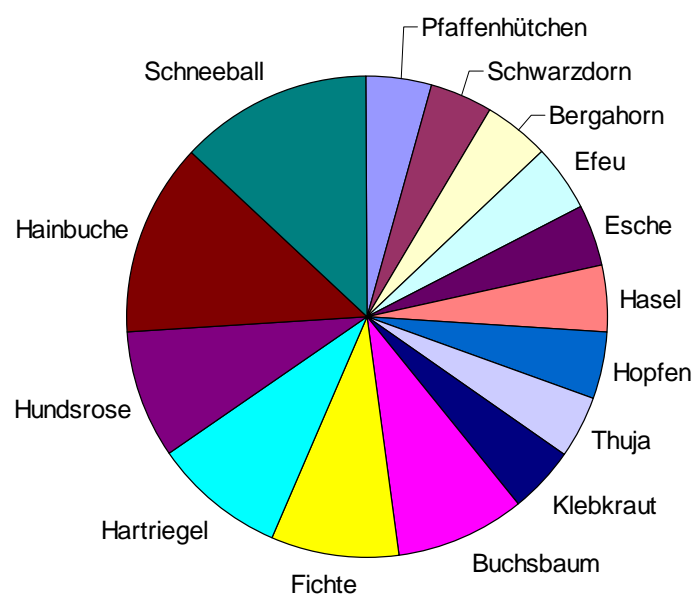

Abb. 36: „Andere“ (vgl. Abb. 35) Nistpflanzen der Mönchsgrasmücke ( $n=23)$.

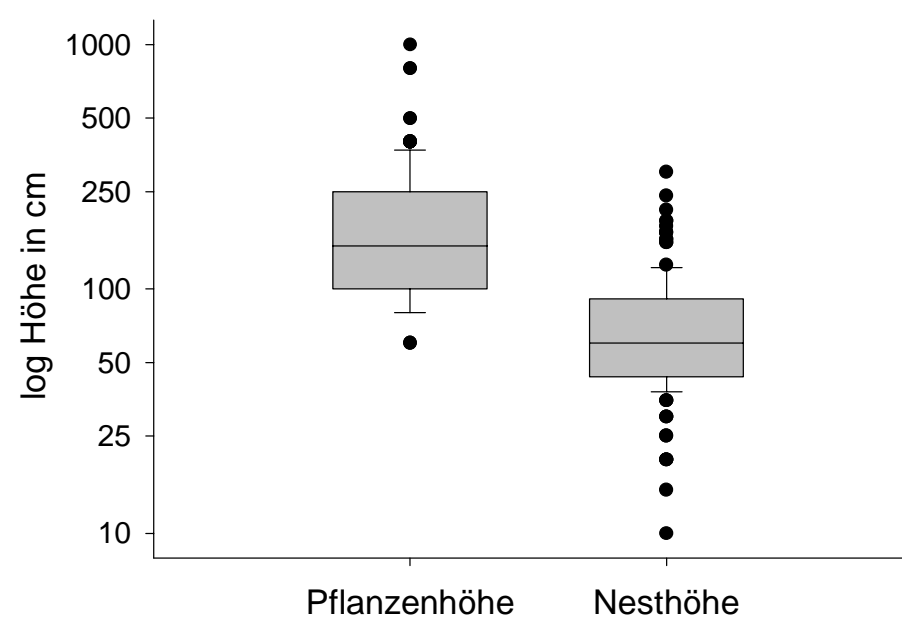

Abb. 37: Höhe der Nistpflanze $(n=121)$ und Nesthöhe ( $n$ = 141), Höhe zur verbesserten Darstellung logarithmiert.

\subsubsection{Neststandortselektion}

\subsubsection{Neststandorte}

Von den 201 Nestern, die im Rahmen der Freilandstudie am Bodensee gefunden wurden, konnte für 152 Nester die jeweilige Nistpflanze festgestellt werden und davon 141 Nester vermessen werden. Insgesamt wurden 25 verschiedene Nistpflanzen genutzt, $41 \%$ verteilten sich auf Brennnessel Urtica dioica und Brombeere Rubus ssp., die restlichen auf zahlreiche verschiedene Baum- und Straucharten sowie rankende Pflanzen (Abb. 35). Neun Pflanzenarten wurden einmal, vier zweimal und zwei dreimal genutzt (Abb. 36).

Die durchschnittliche Höhe der Nistpflanzen betrug $201 \mathrm{~cm}$ (s = $148 \mathrm{~cm}, \mathrm{n}=121$ ), wobei die Verteilung stark rechtsschief war, so dass der Median bei $150 \mathrm{~cm}$ lag. 75 $\%$ der Nester wurden in Pflanzen angelegt, die niedriger waren als $250 \mathrm{~cm}$. Die durchschnittliche Nesthöhe lag bei $75 \mathrm{~cm}$ ( $\mathrm{s}=45 \mathrm{~cm}, \mathrm{n}=$ 141). Auch hier lag der Median wegen einiger sehr hoch gelegener Nester bei nur $60 \mathrm{~cm}$. Drei Viertel der Nester waren in einer Höhe unter $93 \mathrm{~cm}$ angelegt worden (Abb. 
37). Daraus ergab sich die relative Höhe eines Nestes in der Nistpflanze von $42 \%(\mathrm{~s}=0,18, \mathrm{n}=$ 121). Die Nester wurden also im Allgemeinen etwas unterhalb der Mitte einer Neststruktur angelegt (Abb. 38).

Der Mittelwert des Durchmessers der Neststruktur betrug $257 \mathrm{~cm}$ (s $=134 \mathrm{~cm}, \mathrm{n}=54$, Abb. 39). Ohne Brennnessel- und Brombeerfelder betrug der Mittelwert $180 \mathrm{~cm}(\mathrm{~s}=$ $103 \mathrm{~cm}, \mathrm{n}=33$ ). Allerdings wurden 2001 ein Viertel der Nester in flächigen Strukturen gefunden. Die Entfernung des Nestes zum Rand einer Struktur betrug im Schnitt $114 \mathrm{~cm}(\mathrm{~s}=118 \mathrm{~cm}, \mathrm{n}=53$ ), wobei der Median bei $70 \mathrm{~cm}$ lag (Abb. 39). Der Quotient aus der Entfernung vom Nest zum Rand und dem Radius der Struktur zeigt, dass die Nester nicht genau in der Mitte eines Busches angelegt, sondern leicht zum Rand verschoben waren. Setzt man die Fälle, in denen Neststruktur und Entfernung zum Rand der Struktur Werte über $400 \mathrm{~cm}$ hatten, und die Niststruktur demnach mindestens $800 \mathrm{~cm}$ durchmaß, so ergab sich ein Wert von $0,36(s=0,16, n=45)$.

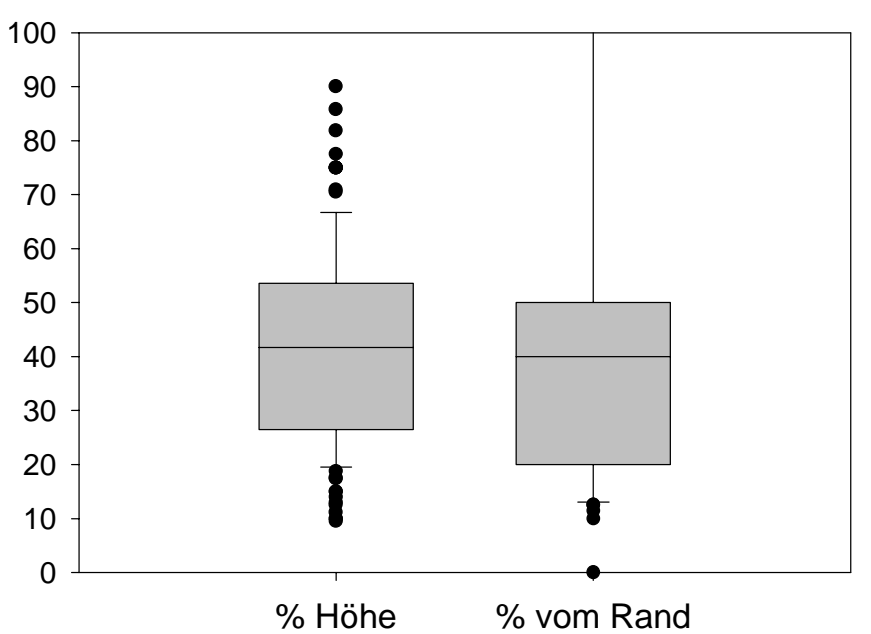

Abb. 38: \% der Nesthöhe von der Nistpflanzenhöhe $(n=121)$ und \% der Entfernung zum Rand vom Radius der Pflanze (n $=54)$.

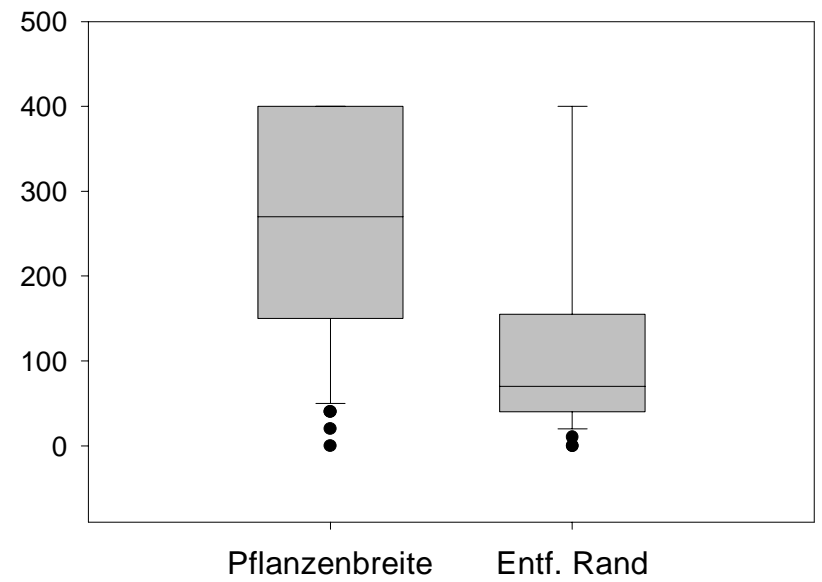

Abb. 39: Breite der Nistpflanzen $(n=54)$ und Entfernung des Nestmittelpunktes zum Rand der Pflanze ( $n=54)$.

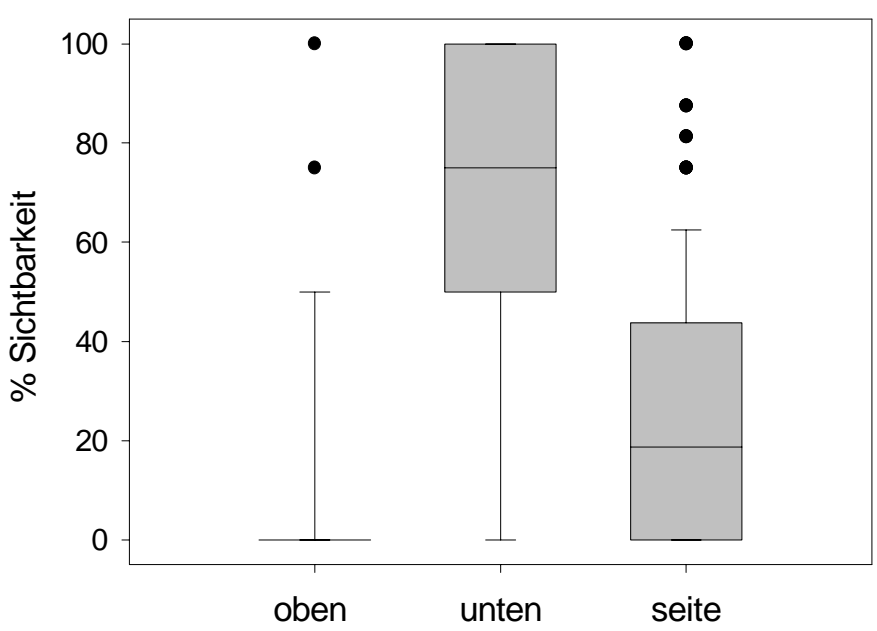

Abb. 40: Sichtbarkeit der Nester von oben $(\mathrm{n}=138)$, von unten $(\mathrm{n}=135)$ und von der Seite (Mittelwert aus den vier gemessenen Richtungen, $\mathrm{n}=138$ ). 


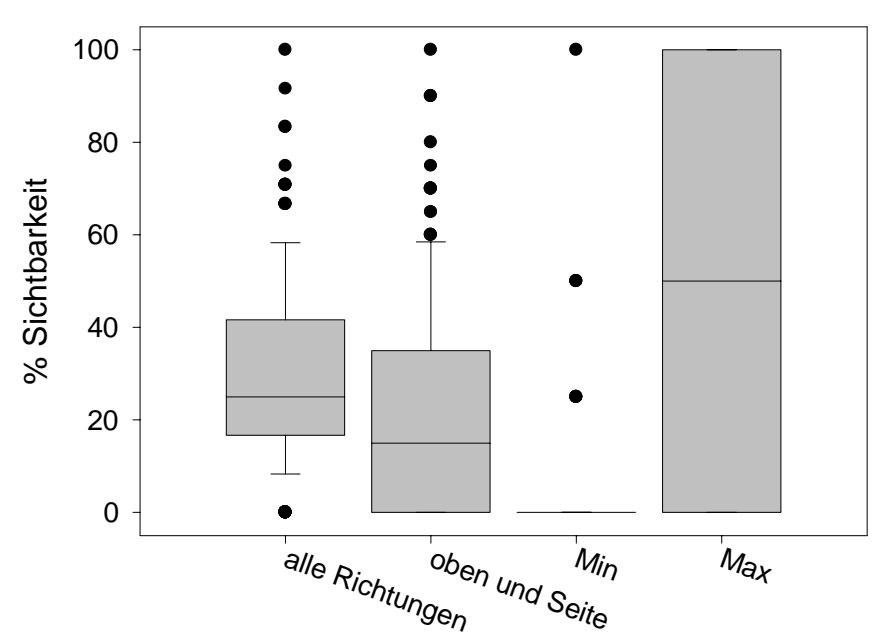

Abb. 41: Mittelwerte der Sichtbarkeit aus allen Richtungen, von oben und der Seite sowie Minimal- und Maximalwerte aus allen Richtungen.

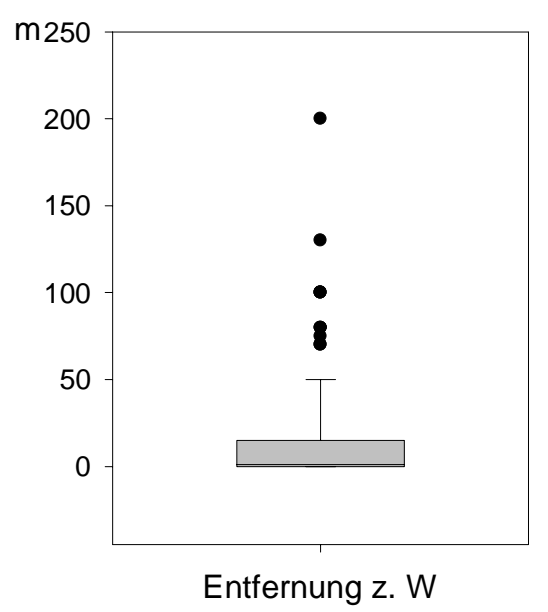

Abb. 42: Entfernung der Nester zum Wandrand in Metern $(\mathrm{n}=$ 137).

Die Sichtbarkeit von der Seite betrug für jede Blickrichtung einzeln gemittelt jeweils etwa $27 \%(n=138)$. Der Median lag jeweils bei 0, d.h. die Hälfte aller Nester waren von der Seite nicht sichtbar. Wurden die Werte aus allen vier Richtungen pro Nest gemittelt, so betrug der Mittelwert dieser "lateralen Sichtbarkeit“ $27 \%$ (s = 26,7, n = 138, Abb. 40), der Median $19 \%$. Der Mittelwert für die Sichtbarkeit von oben betrug $7,4 \%(s=18,2, n=138, A b b .40), 83 \%$ der Nester waren von oben nicht sichtbar.

Dagegen waren sie von unten gut zu sehen, der Mittelwert betrug $74 \%$ ( $s=77 \%$, n = 135, Abb. 40). Berücksichtigte man die Maximalwerte aus allen sechs Richtungen pro Nest, so zeigte sich, dass $10 \%$ der Nester als Maximalwert null aufwiesen, also schon aus einer Entfernung von $1 \mathrm{~m}$ aus keiner Richtung mehr zu sehen waren. Errechnete man den Maximalwert aus den vier Seiten und oben, so erhöhte sich der Prozentsatz auf 30 \%. Die Minimalwerte aus allen sechs Richtungen pro Nest zeigten, dass $94 \%$ aller Nester aus mindestens einer Richtung nicht sichtbar und nur 8 von 138 Nestern aus allen Richtungen sichtbar waren, wobei nur ein Nest aus allen Richtungen voll sichtbar war (Abb. 41).

Die durchschnittlich gemessene Entfernung zum Waldrand betrug 19,2 m. Allerdings lagen 56 \% aller Nester direkt am Waldrand, d.h. innerhalb einer Entfernung von bis zu 5 m. Da vor allem entlang von Rändern gesucht wurde, zeigt dieser Wert keine Präferenzen der Mönchsgrasmücke. Die Verteilung zeigt, dass Mönchsgrasmücken ebenso gut im Waldesinnern brüteten, wenn geeignete Strukturen vorhanden waren (Abb. 42). Die Anzahl der Alternativstrukturen war etwa gleich verteilt auf die 3 Klas- 
sen: je 31 \% für „keine“ und „viele" Alternativstrukturen und $38 \%$ für „einige“.

Es ergab sich für Nesthöhe und Pflanzenarten ein saisonaler Effekt. Die Höhe, in der ein Nest in der Vegetation angelegt wurde, stieg mit der Saison von ca. 60 $\mathrm{cm}$ auf $1 \mathrm{~m}(\mathrm{n}=127, \mathrm{p}<0,0016$, Abb. 43). Die Analyse zeigte, dass dies vornehmlich mit einem abnehmenden Anteil an niedrigen, weniger mit einer Zunahme an hohen Nestern zusammenhing. Früher im Jahr wurden häufig Nester in niedriger Vegetation gefunden, die beim Aufwachsen bodennah zu licht wurde und keine Deckung mehr bot. Diese Entwicklung wird durch die Änderungen in der Pflanzenwahl verdeutlicht (Abb. 44). Immergrüne Pflanzen wie Eibe Taxus baccata, Thuja Thuja occidentalis etc.

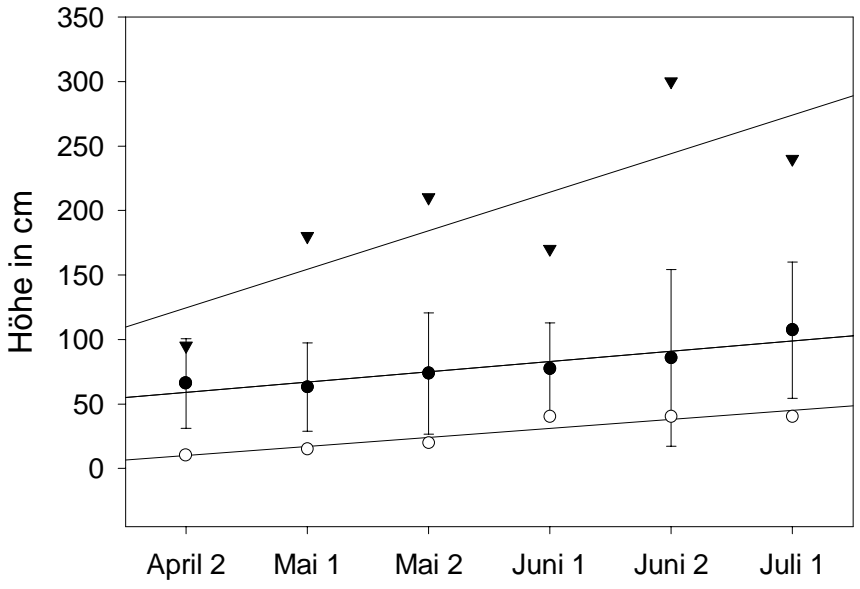

Abb. 43: Zunahme der Nesthöhe mit der Saison. Offene Kreise: Minimal-, Dreiecke: Maximalwerte, geschl. Kreise: Mittelwert mit Standardabweichung.

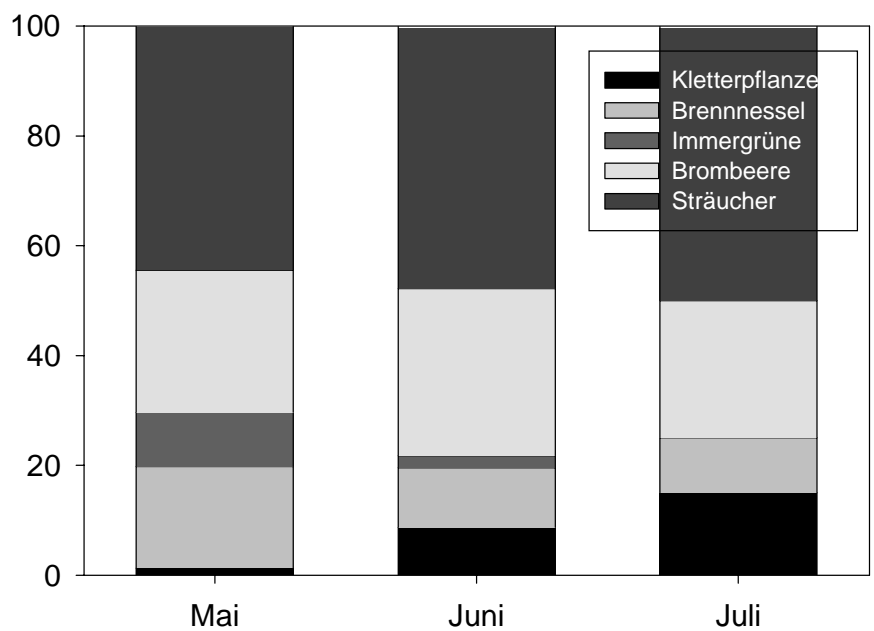

Abb. 44: Änderung der Zusammensetzung der Nistpflanzen in den Monaten Mai, Juni und Juli $\left(n=151, \chi^{2}=22.8, p<\right.$ $0,03)$.

wurden nur zu Beginn der Saison genutzt. Im Juni fanden sich einige Nester in Fichten. Der Anteil an Nestern in Brombeere und Brennnessel nahm ebenfalls ab, wohingegen häufiger Sträucher und Kletterpflanzen genutzt wurden.

\subsubsection{Prädation und Neststandorte}

Der einzige Zusammenhang, der zwischen einem Neststandortparameter und der Prädation belegt werden konnte, war die Analyse selten, d.h. weniger als fünfmal (vgl. Abb. 36 und Schneebeere) und häufig genutzter Nistpflanzen: von 29 Nestern in selten genutzten Strukturen waren nur 9 (31\%), bei 123 Nestern in häufig genutzten 83 (67\%) erfolgreich. Keiner der gemessenen Parameter war mit Erfolg/Misserfolg 
der Nester in Verbindung zu bringen. Weder Höhe, Durchmesser, Entfernung zum Rand der Pflanze oder die verschiedenen Indizes zur Sichtbarkeit erlaubten eine Vorhersage des Bruterfolges. Aus den Werten für die laterale Sichtbarkeit wurde der Mittelwert gebildet, dann der Mittelwert aus den Sichtbarkeitswerten ohne den Wert von unten und schließlich der Mittelwert der Sichtbarkeit aus allen Richtungen. Keiner dieser Mittelwerte zeigte Unterschiede zwischen erfolgreichen und nicht erfolgreichen Nestern. Auch die Position des Nestes in der Pflanze zeigte keine Beziehung zum Bruterfolg (Tab. 14).

Tab. 14: Kennwerte der Tests zum Vergleich erfolgreicher und nicht erfolgreicher Nester hinsichtlich der gemessenen Parameter.

\begin{tabular}{|c|c|c|c|c|c|c|}
\hline Parameter & $\bar{N}$ & Test & Testwert & $\begin{array}{c}\text { Øausge- } \\
\text { flogen }\end{array}$ & $\varnothing$ prädiert & p-Wert \\
\hline Nistpflanze & 152 & $\chi^{2}$-Test & 15,12 & & & 0,94 \\
\hline Nistpflanze Typ (vgl. Abb. 44) & 152 & $\chi^{2 \text {-Test }}$ & 1,06 & & & 0,90 \\
\hline Pflanzenhöhe & 121 & $\mathrm{t}$-Test & $-2,74$ & $205 \mathrm{~cm}$ & $198 \mathrm{~cm}$ & 0,78 \\
\hline Nesthöhe & 141 & $\mathrm{t}$-Test & $-1,54$ & $81 \mathrm{~cm}$ & $70 \mathrm{~cm}$ & 0,13 \\
\hline Pflanze Durchmesser & 54 & t-Test & $-0,33$ & $265 \mathrm{~cm}$ & $253 \mathrm{~cm}$ & 0,74 \\
\hline Nest zum Rand der Pflanze & 53 & t-Test & $-0,82$ & $129 \mathrm{~cm}$ & $102 \mathrm{~cm}$ & 0,41 \\
\hline Alternativstrukturen & 136 & $\chi^{2}$ Test & 1,06 & & & 0,59 \\
\hline Sichtbarkeit von oben & 138 & t-Test & 0,66 & $6,3 \%$ & $8,3 \%$ & 0,51 \\
\hline Sichtbarkeit von unten & 135 & t-Test & $-0,78$ & $70,7 \%$ & $65,6 \%$ & 0,43 \\
\hline Sichtbarkeit Seite & 138 & t-Test & 0,22 & $26,5 \%$ & $27,5 \%$ & 0,76 \\
\hline Sichtb. oben und Seiten & 138 & t-Test & 0,31 & $22,4 \%$ & $23,7 \%$ & 0,82 \\
\hline Sichtbark. alle Seiten & 135 & t-Test & $-0,02$ & $30,1 \%$ & $30,1 \%$ & 0,99 \\
\hline Min Sichtbarkeit & 138 & t-Test & $-0,69$ & $1,9 \%$ & $3,3 \%$ & 0,49 \\
\hline Max Sichtbarkeit & 138 & t-Test & 0,37 & $56,4 \%$ & $53,8 \%$ & 0,71 \\
\hline \% Nesthöhe/Pflanzenhöhe & 121 & t-Test & $-0,78$ & $43 \%$ & $41 \%$ & 0,43 \\
\hline$\%$ Nest zum Rand Nestpfl. & 52 & $\mathrm{t}$-Test & $-0,67$ & $42 \%$ & $39 \%$ & 0,50 \\
\hline
\end{tabular}


Der Test, ob extreme (Werte unterhalb des $10 \%$ bzw. oberhalb des $90 \%$ Quantil) oder mittlere Standorte (Werte zwischen 25 und $75 \%$ Quantil) jeweils einen anderen Bruterfolg haben als die verbleibenden Nester, ergab keine Unterschiede (Tab. 15).

Tab. 15: Kennwerte der Tests zum Vergleich extremer und mittlerer Standorte mit den verbleibenden Nestern.

\begin{tabular}{|c|c|c|c|c|c|c|c|}
\hline Parameter & Wert TG & N TG & $\%$ Erfolg & $\mathbf{N}$ andere & $\%$ Erfolg & $\chi^{2}$ & $\bar{P}$ \\
\hline Sicht Max Q 10 & 0 & 41 & 39 & 97 & 45 & 0,47 & 0,49 \\
\hline Sicht Max Q 90 & 100 & 49 & 37 & 89 & 47 & 1,42 & 0,23 \\
\hline Sicht Max Q 25-75 & $25-75$ & 48 & 54 & 90 & 37 & 84 & 0,36 \\
\hline Sicht Oben Q10 & 0 & 115 & 45 & 23 & 34 & 0,86 & 0,35 \\
\hline Sicht Unten Q 10 & 0 & 24 & 33 & 14 & 46 & 1,24 & 0,27 \\
\hline Sicht Unten Q 90 & 100 & 61 & 44 & 77 & 43 & 0,027 & 0,87 \\
\hline Sicht Unten Q 25-75 & $50-75$ & 47 & 45 & 91 & 43 & 0,024 & 0,84 \\
\hline Sicht Seite Q 10 & 0 & 42 & 40 & 96 & 45 & 0,22 & 0,64 \\
\hline Sicht Seite Q 90 & $>60$ & 23 & 48 & 115 & 43 & 0,21 & 0,65 \\
\hline Sicht Seite Q 25-75 & $1-60$ & 73 & 44 & 65 & 43 & 0,018 & 0,93 \\
\hline Sicht Alle Q 10 & $<8$ & 25 & 44 & 113 & 43 & 0,003 & 0,95 \\
\hline Sicht Alle Q 90 & $>60$ & 13 & 61 & 125 & 42 & 0,5 & 0,83 \\
\hline Sicht Alle Q 25-75 & $16-42$ & 73 & 45 & 65 & 42 & 0,19 & 0,66 \\
\hline Sicht ob.+ Seite Q 10 & 0 & 41 & 39 & 97 & 45 & 2,83 & 0,09 \\
\hline Sicht ob.+Seite Q 90 & $>60$ & 13 & 54 & 125 & 42 & 0,62 & 0,43 \\
\hline $\begin{array}{l}\text { Sicht ob.+Seite Q 25- } \\
75\end{array}$ & $5-35$ & 64 & 48 & 74 & 39 & 1,95 & 0,27 \\
\hline Höhe Pflanze Q 10 & $<90$ & 16 & 44 & 122 & 43 & 0,14 & 0,71 \\
\hline Höhe Pflanze Q 90 & $>350$ & 16 & 37 & 122 & 44 & 0,001 & 0,97 \\
\hline Höhe Pflanze Q 25-75 & $100-250$ & 74 & 39 & 64 & 48 & 1,95 & 0,27 \\
\hline Höhe Nest Q 10 & $<36$ & 14 & 33 & 126 & 56 & 0,36 & 0,56 \\
\hline Höhe Nest Q 90 & $>124$ & 14 & 57 & 126 & 42 & 0,007 & 0,94 \\
\hline Höhe Nest Q 25-75 & $42-93$ & 71 & 41 & 70 & 46 & 0,34 & 0,56 \\
\hline
\end{tabular}




\subsubsection{Prädatoren und Neststandorte}

Beim Vergleich von Neststandorten, an denen Nester von Rabenvögeln oder Säugern ausgeräumt wurden, zeigten sich signifikante Unterschiede in der Höhe der Nester (t-Test, $\mathrm{p}<$ 0,028, $n=32$ ) und in der Randnähe relativ zum Radius der Pflanze ( tTest, $\mathrm{p}<0,016, \mathrm{n}=10)$. Nester, die von Eichelhäher oder Rabenkrähe ausgeräumt wurden, hatten eine mittlere Höhe von $104 \mathrm{~cm}$ (s = 10), die von Säugern ausgefressenen von $60 \mathrm{~cm}(\mathrm{~s}=14)$. Erstere hatten einen relativen Abstand von 0,17 zum Rand der Nistpflanze, letztere von 0,58. Sie lagen somit fast in der Mitte der Nestpfanze. Die Werte für erfolgreiche Nester lagen mit einer mittleren Höhe von $79 \mathrm{~cm}$ (Abb. 45) bzw. einem Wert für die Randnähe von 0,43 (Abb. 46) jeweils dazwischen. Andere Parameter, wie die verschiedenen Indizes zur Sichtbarkeit, zeigten keine Unterschiede. Nach diesen Ergebnissen finden Corviden eher hohe, randständige Nester, Säugetiere dagegen eher niedrige, zentral gelegene. Erfolgreiche Nester ordnen sich zwischen diesen Werten ein.

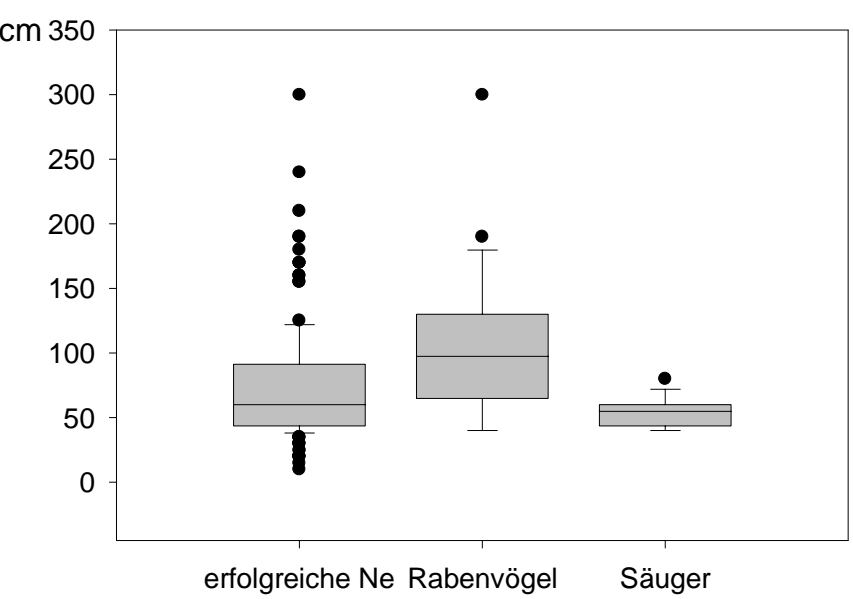

Abb. 45: Box Plots der Nesthöhe erfolgreicher Nester und der Nester, die von Rabenvögeln oder Säugern ausgeräumt wurden (ANOVA, $\mathrm{p}<0,05, \mathrm{n}=91$ ).

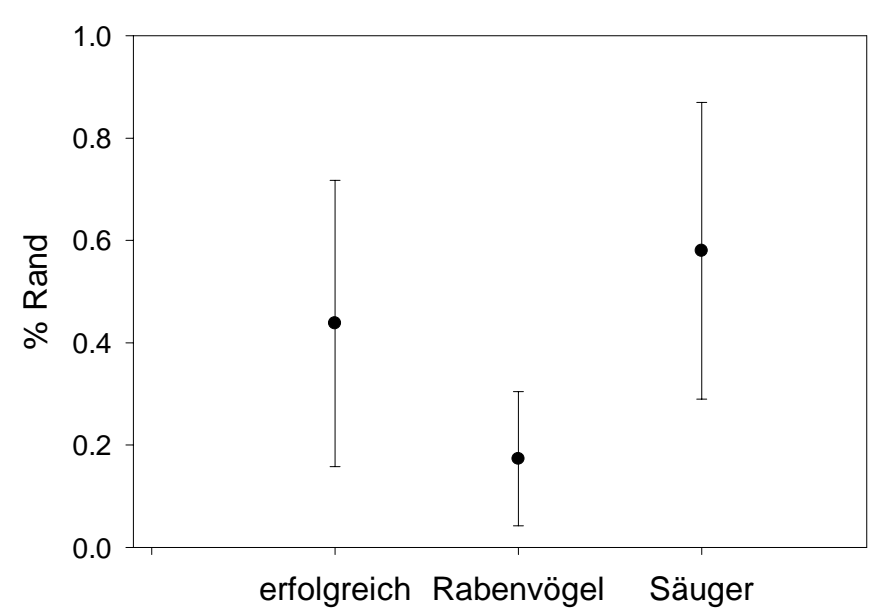

Abb. 46: Mittelwert und Standardabweichung der Randnähe erfolgreicher Nester und der Nester, die von Rabenvögeln oder Säugern geleert wurden (ANOVA, $\mathrm{p}<0,05, \mathrm{n}=$ $30)$. 


\subsubsection{Wahl der Neststandorte und Prädation auf Mallorca}

Auf den beiden Untersuchungsflächen wurden 28 Reviere der Mönchsgrasmücke festgestellt. Damit ergibt sich für die $\mathrm{Be}$ zugsfläche von 55 ha eine Siedlungsdichte von 0,5 Brutpaaren pro ha. Da nur etwa 50 $\%$ dieses Areals bewaldet und damit für die Mönchsgrasmücke nutzbar waren, beträgt die Siedlungsdichte ca. 1 Brutpaar/ha.

Das erste Nest mit 3 Eiern wurde am 17. April 2001 gefunden, das zweite ebenfalls mit drei Eiern am 27. April 2001. Außerdem wurden 64 Vorjahresnester vermessen. Als Hauptnistpflanze diente der Mastixstrauch. Andere Nistpflanzen waren Steineiche, Smilax, Steinlinde und Myrte (Abb. 47). Die Daten zeigen, dass die Wahl weniger flexibel war als in SWDeutschland. So konnte kein Nest in der in Deutschland häufig genutzten Brombeere gefunden werden. Generell entfielen Nistpflanzen, die niedrige und gut versteckte Standorte geboten hätten. Schon auf den ersten Blick unterschieden sich

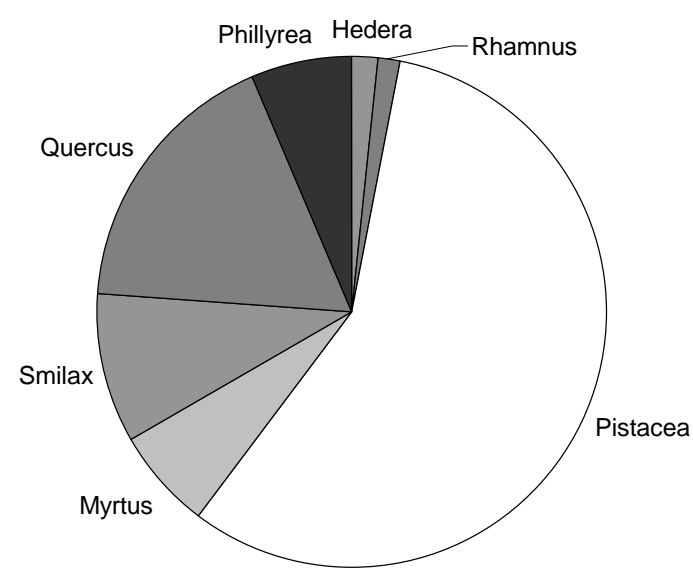

Abb. 47: Nistpflanzen der Mönchsgrasmücke auf Mallorca $(n=64)$

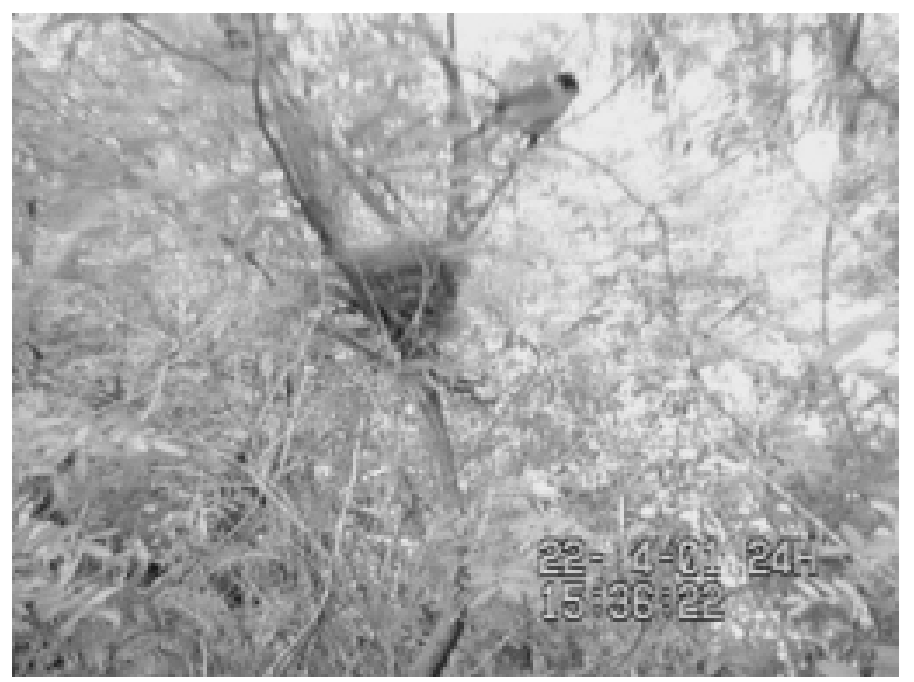

Abb. 48: Nest der Mönchsgrasmücke in einem Mastixstrauch auf Mallorca (aus Videoaufzeichnungen).

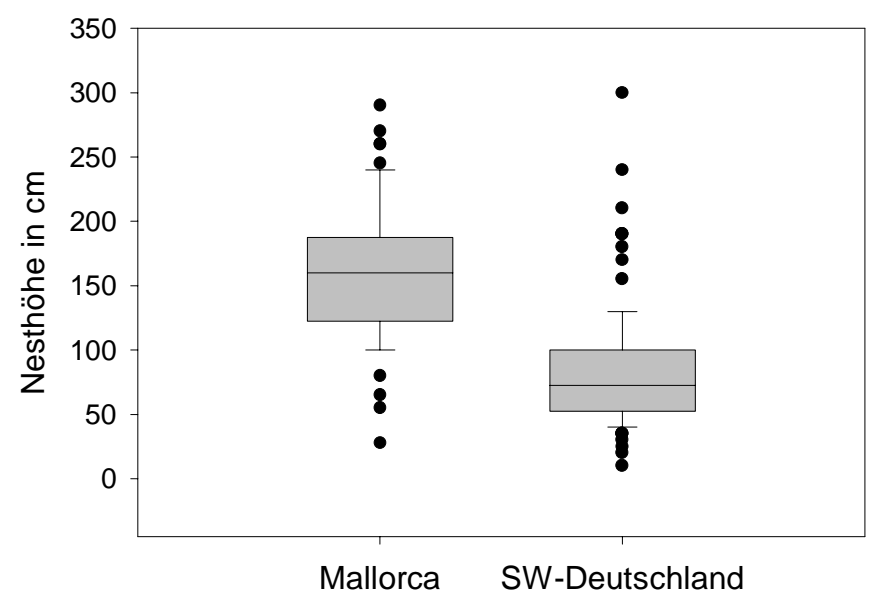

Abb. 49: Höhe von Nestern der Mönchsgrasmücke auf Mallorca $(n=64)$ und in SW-D $(n=96, t-T e s t, p<0,001)$. 


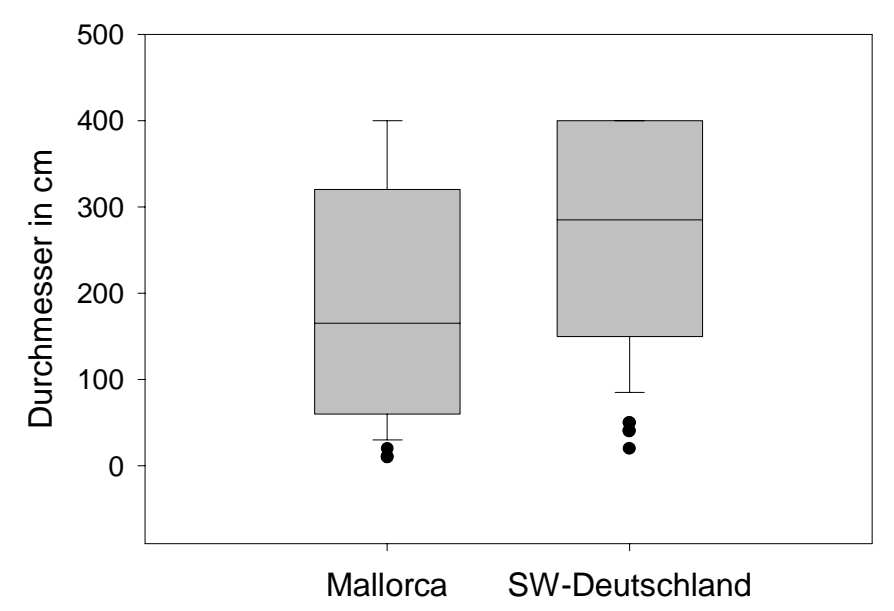

Abb. 50: Breite der Nistpflanze in Nesthöhe auf Mallorca $(\mathrm{n}=64)$ und in SW-D $(\mathrm{n}=52$, t-Test, $\mathrm{p}<0,02)$.

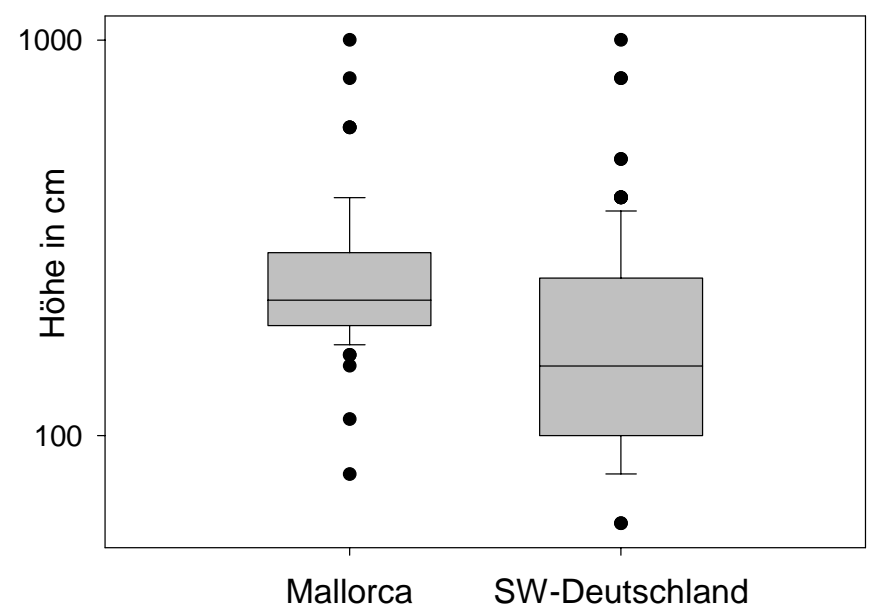

Abb. 51: Höhe von Nistpflanzen der Mönchsgrasmücke auf Mallorca $(\mathrm{n}=64)$ und in SW-D $(\mathrm{n}=96$, $\mathrm{t}$-Test, $\mathrm{p}<$ 0,005).

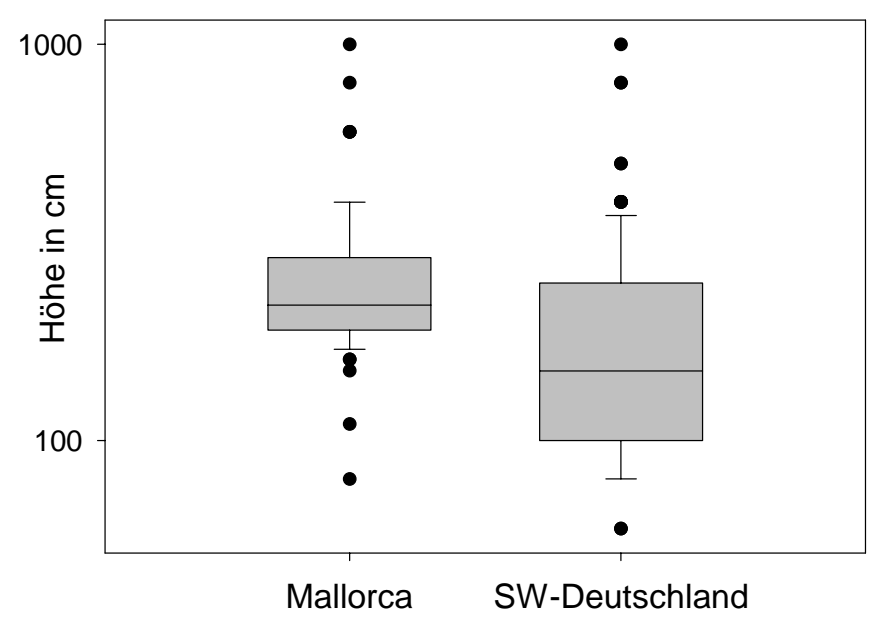

die Neststandorte auf Mallorca von denen in Mitteleuropa (Abb. 48).

Der Vergleich der Daten von Mallorca mit denen aus SWDeutschland zeigt, dass sich die Neststandorte in den gemessenen Parametern deutlich voneinander unterschieden. Nester waren auf Mallorca fast $1 \mathrm{~m}$ höher angelegt als in SW-Deutschland, der Mittelwert lag hier bei $160 \mathrm{~cm}$ (s = 36,7, $n=64$, Abb. 49). Der Nistpflanze auf Mallorca war mit 265 cm ( $\mathrm{s}=150, \mathrm{n}=64)$ höher (Abb. 51) und mit $189 \mathrm{~cm} \mathrm{(} \mathrm{s}=142, \mathrm{n}=$ 64) schmaler (Abb. 50). Mallorquinische und sw-deutsche Nester unterschieden sich auch in der Position innerhalb der Pflanze. Die Mönchsgrasmücke legte ihre $\mathrm{Ne}$ ster auf Mallorca mit $66 \%(s=18$, $\mathrm{n}=64)$ der Pflanzenhöhe deutlich oberhalb der Mitte einer Nistpflanze an (Abb. 52) und die Nester lagen dort wesentlich näher am Rand einer Struktur (27\%, s = 15,4, $\mathrm{n}=63$, Abb. 53). Während der Nestersuche zeigte sich, dass Mönchsgrasmückennester hier schon aus

Abb. 52: Relative Höhe von Nestern der Mönchsgrasmücke größerer Entfernung gut sichtbar auf Mallorca $(n=64)$ und in SW-D $(n=52, t-T e s t, p<$ waren. Die Werte der Sichtbar0,001). 
Tab. 16: Erfolgreiche (e) und nicht erfolgreiche (n) Fotofallennester auf Mallorca und in SWDeutschland $\left(\mathrm{X}^{2}=12,5, \mathrm{p}<\right.$ $0,0004)$.

\begin{tabular}{|llc|}
\hline & $\mathrm{E}$ & $\mathrm{N}$ \\
\hline Mallorca & 12 & 0 \\
\hline SW-D & 29 & 37 \\
\hline
\end{tabular}

Tab. 17: Erfolgreiche (e) und nicht erfolgreiche (n) Kunstnester auf Mallorca und in SW-Deutschland im Juni $\left(X^{2}=17,6, p<0,0001\right)$ und Juli $\left(X^{2}=11,7, p<0,0006\right)$.

\begin{tabular}{|lrr|}
\hline & $\mathrm{E}$ & $\mathrm{N}$ \\
\hline Mallorca & 20 & 0 \\
\hline SW-D Juni & 9 & 11 \\
\hline SW-D Juli & 12 & 8 \\
\hline
\end{tabular}

keit von oben und vom Mittelwert der vier lateralen Werte bestätigten diesen Eindruck:

Der Mittelwert für die Sichtbarkeit von

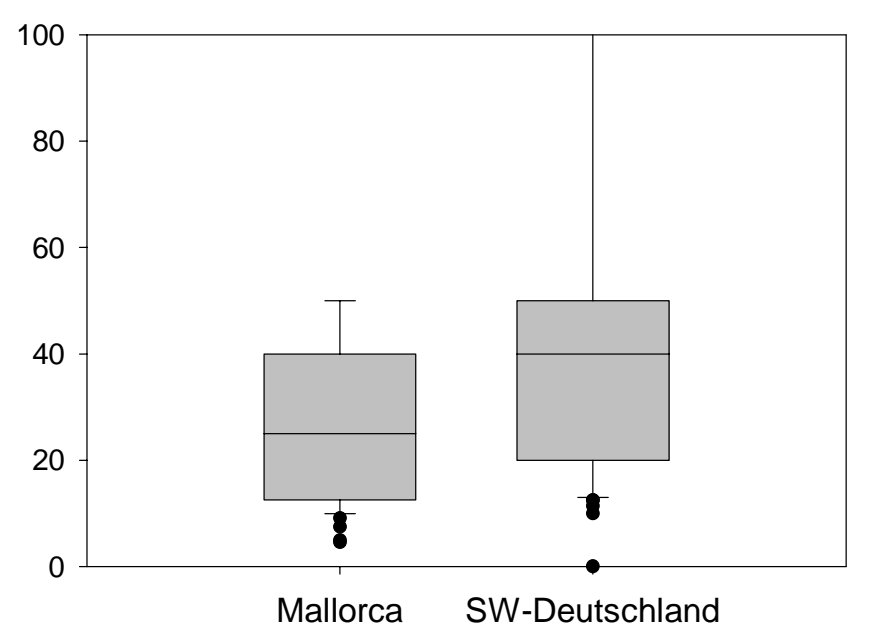

Abb. 53: Relative Nestentfernung zum Rand der Nistpflanze auf Mallorca $(\mathrm{n}=63)$ und in SW-D $(\mathrm{n}=51$, t-Test, $\mathrm{p}<$ $0,001)$.

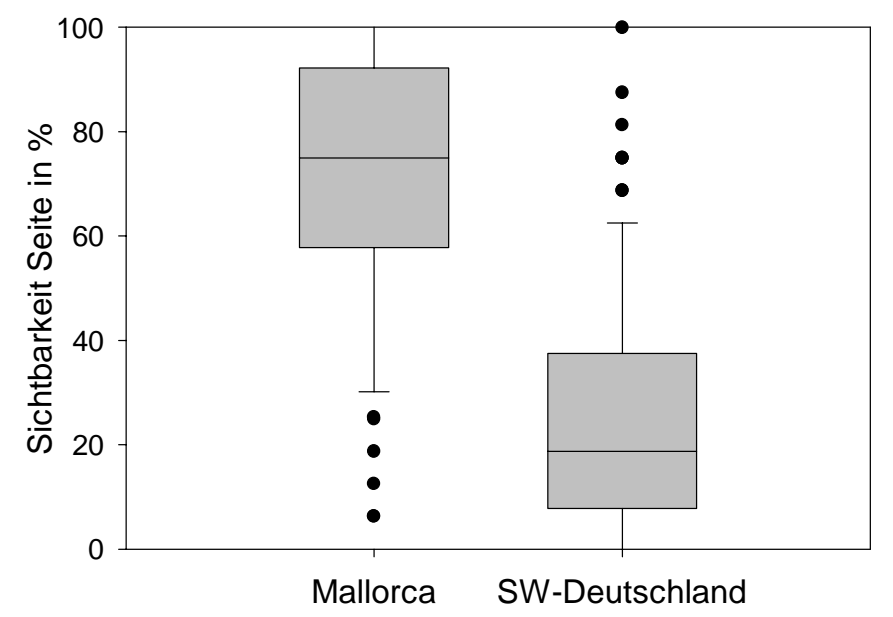

Abb. 54: Laterale Sichtbarkeit von Nestern auf Mallorca $(\mathrm{n}=59)$ und in SW-D ( $=$ = 91, t-Test, $\mathrm{p}<0,001)$. oben betrug $42 \%(s=12,9, n=59)$ und unterschied sich damit von den Werten aus SW-Deutschland (t-Test, $\mathrm{p}<0,001)$. Die mittlere Sichtbarkeit von der Seite betrug $70,9 \%(s=10,6, n=59)$ und ist damit ebenfalls höher als in SW-Deutschland (Abb. 54).Mit keiner der angewandten Methoden konnten Prädation oder Prädatoren nachgewiesen werden. Die Anzahl videoüberwachter Nester war mit 11 recht gering, da alle bis zum Ende der Beobachtungen erfolgreich und die Kameras somit gebunden waren. Doch auch bei den übrigen Nestern wurde keine Prädation festgestellt. Auch bei den Fotofallen (240 Fallentage) und den Kunst-nestern (210 Nesttage) wurden keine Eier entnommen. Nach Anzahl erfolgreicher und nicht erfolgreicher Nester getestet, ergibt sich im Vergleich mit SW-Deutschland für jede der Methoden ein signifikanter Unterschied (Tab. 16, Tab. 17). Die Prädation auf Kunstnester und Fotofallen ist am Bodensee höher. 


\subsection{Reduktion des Prädationseffektes}

\subsubsection{Kompensation von Gelegeverlusten}

Um zu ermitteln, wie schnell Mönchsgrasmücken Gelegeverluste kompensieren können, wurden Daten aus den Volieren ausgewertet. Insgesamt konnten 216 Bruten und 122 Übergänge zwischen Bruten bestimmt werden. Die mittlere Überlebensdauer der Volierennester stimmte mit $11,1(s=2,6, n=216)$ mit der im Freiland überein (Abb. 55). Die Zeit zwischen zwei Brutversuchen reichte von 3 bis zu 28 Tagen, der Modalwert (35\% aller Werte) lag bei 5 Tagen. Im Mittel benötigten die Mönchsgrasmücken in den Volieren 7,4 Tage $(s=4,3, n=122)$ bis zum Legebeginn in einem

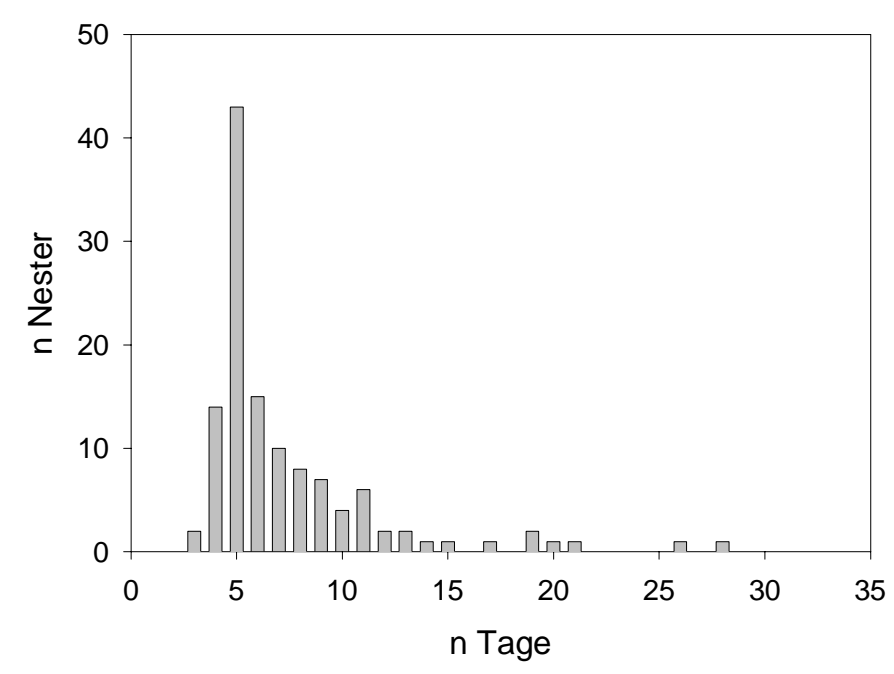

Abb. 55: Nachlegedauer in den Volieren $(\mathrm{n}=122)$.

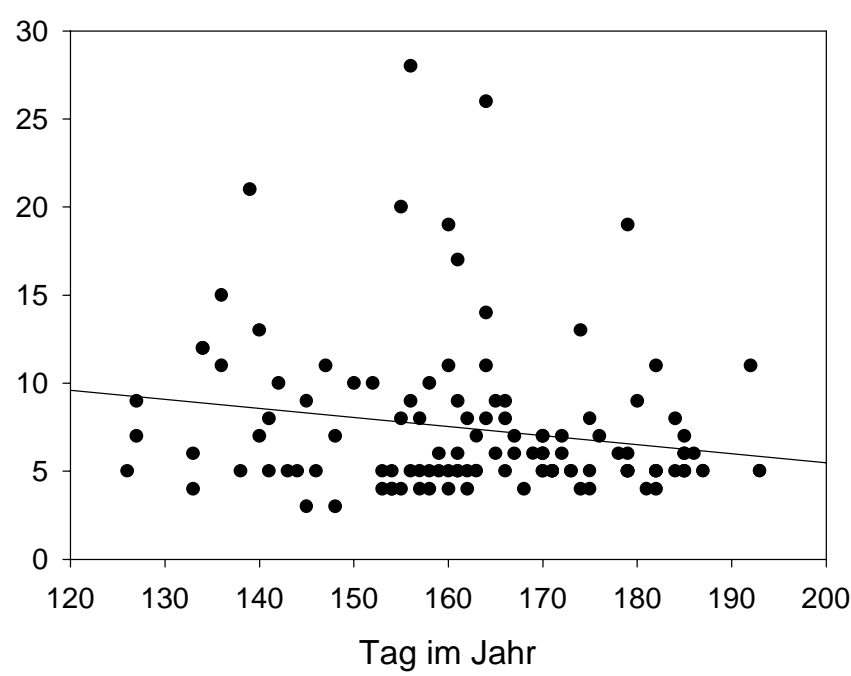

Abb. 56: Saisonale Abhängigkeit der Nachlegedauer in den Volieren $\left(r^{2}=0,034, p<0,03, n=122\right)$.
Nachgelege, wegen einiger sehr hoher Werte liegt der Median bei 6 Tagen. Die Werte decken sich mit langjährigen Freilandbeobachtungen, nach denen etwa eine Woche vergeht, bis in einem Brutrevier ein Nachgelege gefunden werden kann (QUERNER, mündl.). Dieser Wert blieb in den Volieren über die Saison nicht konstant, sondern sank von etwa 10 Tagen Anfang Mai auf 5 Tage Anfang Juli (Abb. 56). Dieser Trend war allen Vögel gemeinsam, fand sich aber auch bei der Betrachtung aufeinanderfolgender Übergänge bei einzelnen Brutpaaren. Je häufiger ein Paar brütete, desto weniger Zeit verging zwischen den Versuchen. Waren es zwischen dem ersten und zweiten Brutversuch 8,6 Tage, verkürzte sich die Zeit zwischen viertem und fünftem auf 
nur noch 4,8 Tage (Abb. 57).

Die theoretisch mögliche Anzahl von Brutversuchen pro Jahr betrug demnach 4,4 und errechnete sich aus 10,5 Tagen Überlebensdauer für ein nicht erfolgreiches Nest (4.1) und 6 (Median) Tagen, die zwischen zwei Brutversuchen vergingen, sowie der Dauer der Brutsaison von 72,6 Tagen (s.u.). Unter Einbeziehung der $25 \%$ und 75

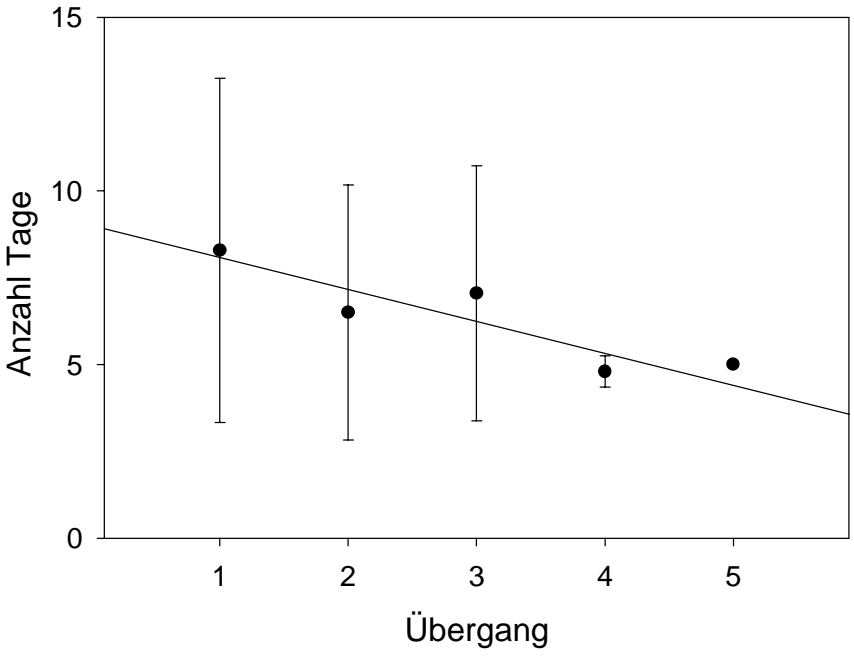

Abb. 57: Nachlegedauer in Abhängigkeit der Brutversuche eines Paares (ANOVA, $\mathrm{p}<0,023, \mathrm{n}=122$ ). $\%$ Quantile ergaben sich für eine kurze Brutsaison (72,6 - s) mit langer Überlebensdauer $(10,5+s)$ und langen Übergängen $(8,25)$ zwischen den Brutversuchen 2,4 und für eine lange Brutsaison $(72,6+$ s), kurze Überlebensdauer (10,5 - s) und kurze Übergangszeiten (5 Tage) 8,5 Brutversuche.

\subsubsection{Brutbiologie, Gelegegröße und Überlebenswahrscheinlichkeit}

Der früheste aus den Nestfunden errechnete Legebeginn war im Jahr 1994 der 7. April, das Nest war am 29. April mit siebentägigen Jungen gefunden worden. Abb. 58 zeigt, dass der früheste festgestellte Termin von Jahr zu Jahr schwankte. Der Median für den frühesten Legebeginn lag zwischen 1981 und 2000 auf dem 28. April. Auch der Median des Legebeginns der Nester eines Jahres schwankte von Jahr zu Jahr und lag über 20 Jahre gemittelt drei Wochen nach dem frühesten Legebeginn auf dem 20. Mai. Die spätesten festgestellten Legebeginne hatten ihren Median auf dem

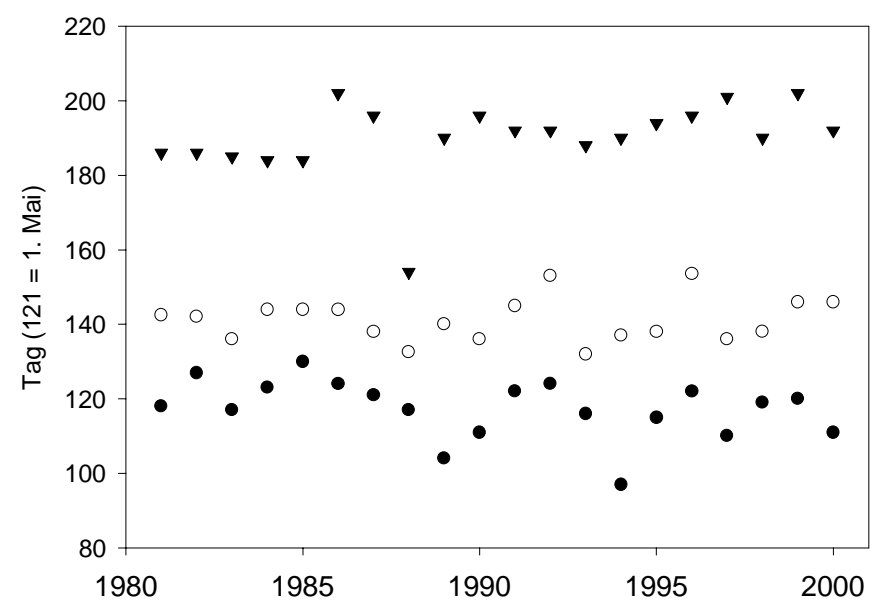
10. Juli, vier Nester hatten am 21. Abb. 58: Früheste (schwarze Kreise) und späteste LegebeJuli 1986 ihren Legebeginn. Die Dauer der Brutsaison betrug über ginne (Dreiecke) eines Jahres sowie Median des Legebeginns (weiße Kreise) der Nestfunde seit 1981. 


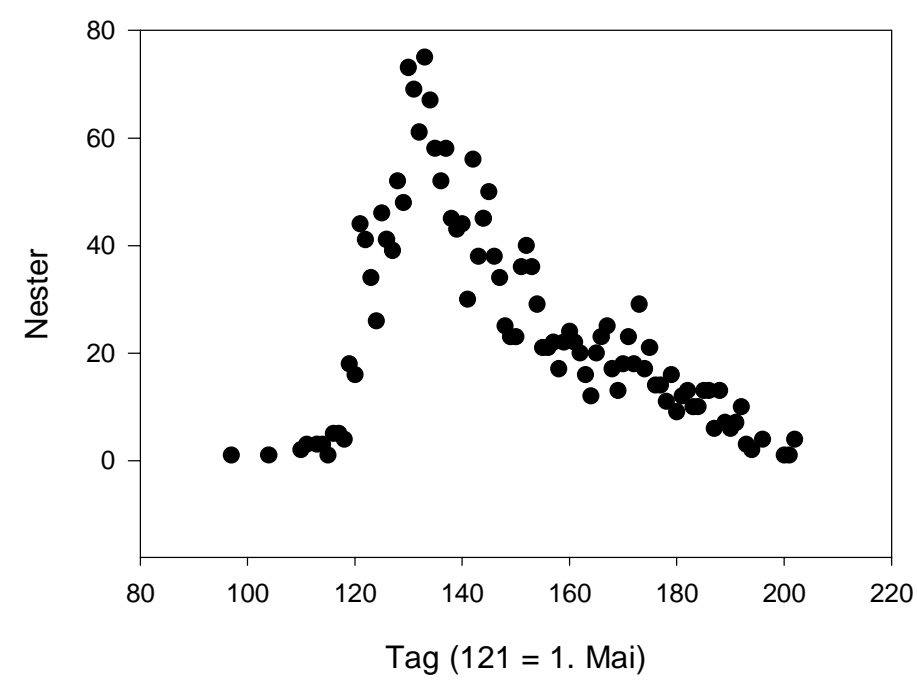

Abb. 59: Anzahl der Legebeginne pro Tag $(n=2201)$ aus den Nestfunden.

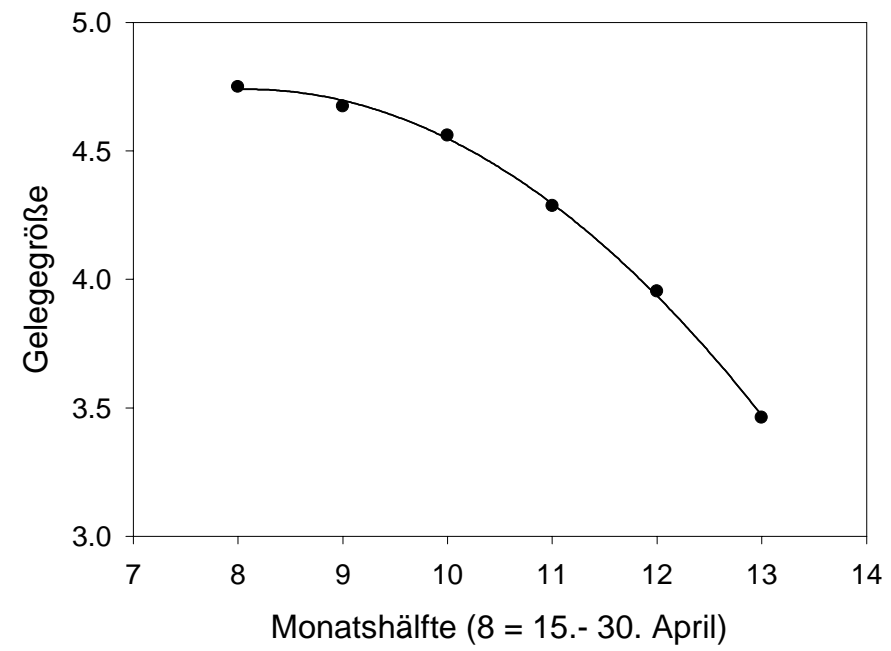

Abb. 60: Saisonale Abhängigkeit der Gelegegröße nach Mittelwerten in einer Monatshälfte aus der Freilandstudie.

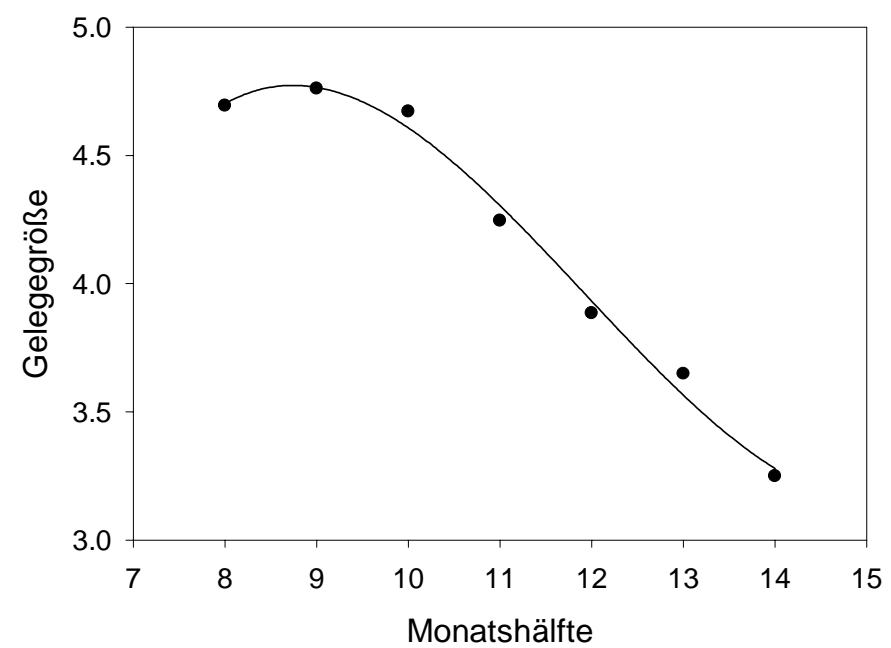

Abb. 61: Saisonale Abhängigkeit der Gelegegröße nach Mittelwerten in einer Monatshälfte aus den Nestfunden.
20 Jahre gemittelt 72,6 Tage ( $\mathrm{s}=$ 13.2, $n=20)$. Der Verlauf des Legemusters, d.h. die Verteilung der Legebeginne an einzelnen Tagen im Jahr (Abb. 59), zeigt eine stark rechtsschiefe Verteilung. Der Anstieg bis zum Gipfel, der um den Median lag, dauerte lediglich 3 Wochen. Bis dahin waren bereits die Hälfte aller Eier gelegt. Der dann folgende Abfall bis zum Ende der Brutsaison erstreckte sich über die dreifache Zeit. Die Daten zeigen einen weiteren kleinen Gipfel Ende Juni, der auf einen geringen Anteil an Zweitgelegen hindeutet.

Der früheste und späteste in der Freilandstudie festgestellte Legebeginn war der 18. April 2000 bzw. 18. Juli 1999. Die Spanne von frühesten zum spätesten Legebeginn beträgt also 91 Tage, in den einzelnen Jahren waren es zwischen 70 und 80 Tagen (Mittel: 74,3 Tage). Der Median der Legebeginne lag auf dem 22. Mai $(\mathrm{n}=$ 172). Klammert man das erste Jahr (1999) wegen anfänglicher Schwierigkeiten bei der Nestersuche 1999 aus, ergibt sich der 15. Mai $(n=155)$.

Die mittlere Gelegegröße der wäh- 
rend der Freilandstudie gefundenen Nester betrug 4,39 ( $s=0,68, n=155)$. Es zeigte sich ein deutlicher Kalendereffekt der Gelegegröße. Abb. 60 zeigt diese Abhängigkeit anhand der Mittelwerte in jeder Monatshälfte. Für einen kubischen Zusammenhang ergibt sich ein $r^{2}$ von 1 . Die Mönchsgrasmücke begann mit einer Gelegegröße von 4,75 in der zweiten Aprilhälfte, die Anzahl der Eier pro Nest sank dann bis auf 3,46 in der ersten Julihälfte.
Tab. 18: Anzahl und Auftretenswahrscheinlichkeit verschiedener Gelegegrößenklassen der Nestfunde $(n=1424)$.

\begin{tabular}{|crr|}
\hline Gelegegröße & Anzahl & \multicolumn{1}{c|}{$\%$} \\
\hline 2 & 14 & 1 \\
\hline 3 & 104 & 7 \\
\hline 4 & 494 & 35 \\
\hline 5 & 779 & 55 \\
\hline 6 & 33 & 2 \\
\hline Summe & $\mathbf{1 4 2 4}$ & $\mathbf{1 0 0}$ \\
\hline
\end{tabular}

Die durchschnittliche Gelegegröße der Nestfunde betrug 4,5 ( $\mathrm{s}=0,71, \mathrm{n}=1424$ ). Wie Tab. 18 zeigt, bestanden 90 \% aller Vollgelege aus 4 oder 5 Eiern. Die Dezimale gibt demnach das Verhältnis zwischen diesen Gelegegrößen an. Auch bei diesen Daten zeigte sich eine saisonale Abhängigkeit. Die frühesten Gelege einer Saison hatten 4 Eier, dann stieg der Wert im Mai auf 5 und fiel bis Mitte Juli auf 3 ab (Abb. $61)$.

Die Gelegegröße zeigt nicht nur zeitliche, sondern auch lokale Unterschiede. Sie ist in den untersuchten Waldstücken verschieden (Tab. 19). Dieser Effekt bleibt bestehen, wenn man saisonale Effekte und Unterschiede zwischen den Jahren berücksichtigt (Tab. 20). Nach dieser Korrektur für den Legebeginn sind die Gelege in „Espasingen I“ signifikant größer, im „Großer Moos“, „Mooswald“ und „Schloss“ klei-

Tab. 19: Mittelwerte und Standardfehler der Gelegegröße in den Untersuchungsflächen des Nestfundes. Die Flächen unterscheiden sich hinsichtlich der Gelegegröße (ANOVA, $\mathrm{p}<0,001)$

\begin{tabular}{|lccc|}
\hline Ort & Anzahl & Mit- & SE \\
& & Nester & tel- \\
wert & \\
\hline Böhringen & 272 & 4,60 & 0,04 \\
\hline Erdbeerwald & 100 & 4,38 & 0,07 \\
\hline Espasingen I & 313 & 4,67 & 0,04 \\
\hline Espasingen II & 234 & 4,53 & 0,05 \\
\hline Großer Moos & 193 & 4,26 & 0,05 \\
\hline Mooswald & 175 & 4,42 & 0,05 \\
\hline Schloss & 137 & 4,41 & 0,06 \\
\hline
\end{tabular}

Tab. 20: Unterschiede in der Gelegegröße zwischen den Untersuchungsflächen unter Berücksichtigung der Kovariaten "Legebeginn" und "Jahr" (ANCOVA, $\mathrm{p}<0,001)$.

\begin{tabular}{|c|c|c|c|c|}
\hline & $\begin{array}{l}\text { Schät- } \\
\text { zer }\end{array}$ & SE & t-Ratio & $\mathrm{P}<(\mathrm{t})$ \\
\hline Böhringen & $-0,030$ & 0,037 & $-0,82$ & 0,414 \\
\hline Erdbeerwald & 0,095 & 0,057 & 1,66 & 0,096 \\
\hline Espasingen I & 0,139 & 0,038 & 3,72 & $<0,01$ \\
\hline Espasingen II & 0,0247 & 0,042 & 0,58 & 0,56 \\
\hline Großer Moos & $-0,098$ & 0,046 & $-2,15$ & $<0,05$ \\
\hline Mooswald & 0,095 & 0,048 & 1,99 & $<0,05$ \\
\hline Schloss & $-0,226$ & 0,049 & $-4,62$ & $<0,01$ \\
\hline Jahr & $-0,132$ & 0,037 & $-3,60$ & $<0,01$ \\
\hline Legebeginn & $-0,873$ & 0,042 & $-20,59$ & $<0,01$ \\
\hline
\end{tabular}




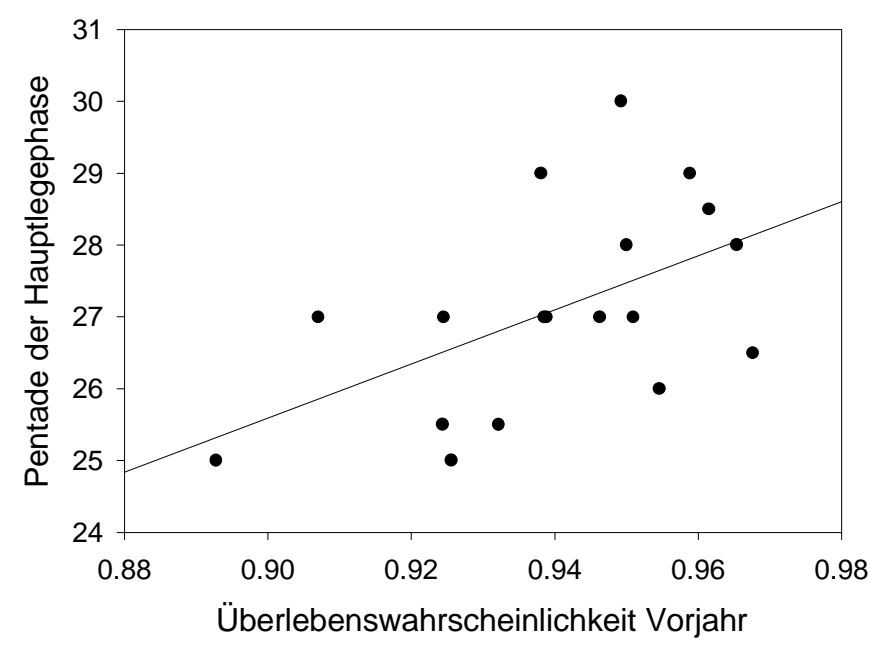

Abb. 62: Zusammenhang zwischen der Hauptlegephase und der Überlebenswahrscheinlichkeit im Vorjahr $\left(r^{2}=\right.$ $0,29, \mathrm{p}<0,024)$.

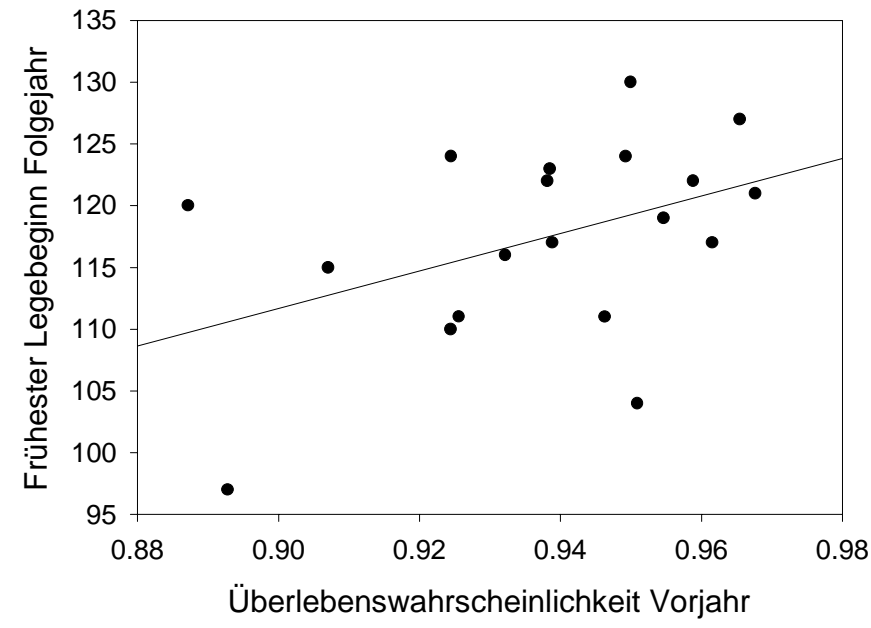

Abb. 63: Zusammenhang zwischen frühestem Legebeginn und Überlebenswahrscheinlichkeit im Vorjahr $\left(\mathrm{r}^{2}=0,19\right.$, n.s.).

Tab. 21: Kennwerte der Multiplen Regression für die Abhängigkeit der Hauptlegephase vom Jahr und der täglichen Überlebenswahrscheinlichkeit (tÜ) und Werte der Power-Analyse (KSW: kleinster signifikanter Wert, $\mathrm{n}=20$ ).

\begin{tabular}{|crrrlll|}
\hline Variable & DF & QS & F-Rat & $p$ & Power & KSW \\
\hline Jahr & 1 & 48,2 & 0,96 & 0,34 & 0,15 & 74 \\
\hline tÜ & 1 & 175,9 & 3,51 & 0,081 & 0,42 & 22,39 \\
\hline
\end{tabular}

Tab. 22: Kennwerte der Multiplen Regression für die Abhängigkeit des frühesten Legebeginnes vom Jahr und von der täglichen Überlebenswahrscheilichkeit (tÜ) und Werte der Power-Analyse (s.o.).

\begin{tabular}{|cccclcc|}
\hline VARIABLE & DF & QS & F-RAT & P & POWER & KSW \\
\hline Jahr & 1 & 1,01 & 0,63 & 0,44 & 0,12 & 112 \\
\hline tÜ & 1 & 4,93 & 3,08 & 0,099 & 0,38 & 25,13 \\
\hline
\end{tabular}

ner, „Böhringen“, „Erdbeerwald“ und „Espasingen II" zeigen keine signifikanten Unterschiede.

Als Kennwerte für die zeitliche Einordnung der Brutsaison wurden der früheste Legebeginn und die Hauptlegephase (Modal der nach Pentaden eingeteilten Legebeginne) definiert. Es besteht ein signifikanter statistischer Zusammenhang zwischen der Hauptlegephase und der Prädation im Vorjahr: wenn die Prädation hoch war, ist die Hauptlegephase früher (Abb. 62). Für den frühesten Legebeginn ergibt sich eine Tendenz, aber kein signifikanter Zusammenhang (Abb. 63), der in die gleiche Richtung weist. Je höher die Prädation im Vorjahr, desto eher der früheste Legebeginn im Folgejahr. Wenn das Jahr als Einflussgröße in einer multiplen Regression berücksichtigt wird, sind diese Zusammenhänge nicht signifikant (Tab. 21, Tab. 22).

Um festzustellen, ob mit den vorliegenden Testwerten ein signifikanter Zusammenhang zu erwarten wäre, wurde eine 
Power-Analyse durchgeführt. Die Power für die Überlebenswahrscheinlichkeit liegt für den frühesten Legebeginn bei 0,42 und für die Hauptlegephase bei 0,38, signifikante Unterschiede waren demnach nicht zu erwarten. Bei einer Stichprobengröße von 26 (frühester Legebeginn) bzw. 23 (Hauptlegephase) würden unter den vorliegenden Voraussetzungen die Tendenzen auf einem Niveau von 0,05 signifikant.

Für einen Zusammenhang zwischen der Prädationsrate und der Gelegegröße lassen sich aus den Nestfunden zwei Ergebnisse darstellen: Zunächst wurden lokale Unterschiede zwischen den Waldstücken sowohl in der Gelegegröße als auch in der über die Jahre gemittelten täglichen Überlebenswahrscheinlichkeit nachgewiesen. Setzte man diese Unterschiede durch eine logistische Regression in Zusammenhang, so ergab sich, dass sich die Gelegegrößenanteile mit zunehmender Überlebenswahrscheinlichkeit änderten: Die Gelege waren in Waldstücken mit hoher Überlebenswahrscheinlichkeit größer. Über eine logistische Regression wurden die Parameter „Jahr“ und „Legebeginn“ als mögliche Einflussgrößen konstant gehalten. Auch nach dieser Analyse war die mittlere tägliche Überlebenswahrscheinlichkeit in einem Waldstück ein Prädikator für die Gelegegröße (Tab. 23).

Schließlich wurde die Abhängigkeit der Gelegegröße von der Überlebenswahrscheinlichkeit im Vorjahr untersucht. Danach gibt es einen statistischen Zusammenhang zwischen der Überlebenswahrscheinlichkeit in einem und der Gelegegröße im Folgejahr (n.s.). Die Korrelation ist stärker, wenn man nicht
Tab. 23: Kennwerte der logistischen Regression zwischen Gelegegröße und täglicher Überlebenswahrscheinlichkeit (tÜ) in einem Waldstück, dem Fundjahr und dem Legebe$\operatorname{ginn}(n=1492)$.

\begin{tabular}{|c|c|c|}
\hline TERM & $\begin{array}{c}\text { SCHÄTZE SE TRATIO } \\
\text { R }\end{array}$ & PROB $>$ \\
\hline tÜ & 3.9291 .3782 .85 & 0.0044 \\
\hline Jahr & $-0.0120 .003-3.78$ & 0.0002 \\
\hline Legebeginn & $-0.0180 .001-19.82$ & $<.0001$ \\
\hline
\end{tabular}

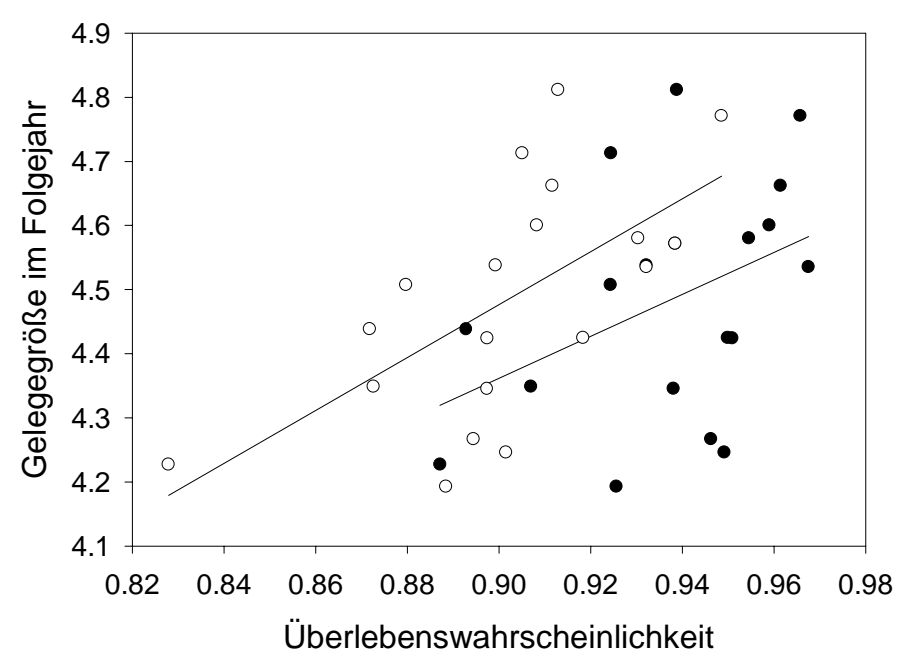

Abb. 64: Zusammenhang zwischen der Gelegegröße und der Prädationsrate im Vorjahr (schwarze Kreise: Natürliche Prädation, $\mathrm{r}^{2}=0,16, \mathrm{p}<0,0823$; offene Kreise nach Entnahme von Versuchsvögeln, $r^{2}=0,38, p<0,0046$. 
Tab. 24: Kennwerte der logistischen Regression zwischen Kalendereffekt, natürliche Überlebenswahrscheinlichkeit und der Gelegegröße als abhängiger Variable (n $=20$ ).

\begin{tabular}{|lll|}
\hline & $\chi^{2}$ & $\mathrm{P}$ \\
\hline Überlebensw. & 3,98 & $<0,05$ \\
\hline Kalendereff. & 7,2 & $<0,01$ \\
\hline
\end{tabular}

Tab. 25: Kennwerte der logistischen Regression zwischen Kalendereffekt, Überlebenswahrscheinlichkeit nach Entnahme von Versuchsvögeln und der Gelegegröße als abhängiger Variable $(\mathrm{n}=20)$.

\begin{tabular}{|c|c|c|}
\hline & $\chi^{2}$ & $\bar{P}$ \\
\hline Überlebensw. & 8,04 & $<0,01$ \\
\hline Kalendereff. & 7,44 & $<0,01$ \\
\hline
\end{tabular}

Tab. 26: Tägliche Überlebenswahrscheinlichkeit (tÜ) der einzelnen Gelegegrößenklassen.

\begin{tabular}{|crr|}
\hline Gelegegröße & $\mathrm{N}$ & $\mathrm{tU}$ \\
\hline 2 & 13 & 0.941 \\
\hline 3 & 85 & 0.952 \\
\hline 4 & 349 & 0.962 \\
\hline 5 & 509 & 0.961 \\
\hline 6 & 16 & 0.959 \\
\hline
\end{tabular}

die natürliche, sondern die tatsächliche Prädation berücksichtigt, also auch die Entnahme von Nestlingen für Versuchszwecke (Abb. 64). Ein Teil der Varianz der Gelegegröße eines Jahres erklärt sich damit aus der Überlebenswahrscheinlichkeit im Vorjahr. Wenn die Überlebenswahrscheinlichkeit hoch war, wurden im Folgejahr mehr Eier gelegt (Abb. 64).

Dieser Effekt wird bestätigt, wenn über ein logistisches Modell mit den Residuen der Gelegegröße der Kalendereffekt berücksichtigt wird (Tab. 24, Tab. 25). Abschließend wurde untersucht, ob ein Zusammenhang zwischen der Anzahl der Eier in einem Nest und der Überlebenswahrscheinlickeit besteht, also ob die Prädationsrate gelegegrößenabhängig ist (Tab. 26). Gelege mit 4, 5 und 6 Eiern zeigen etwas höhere Überlebenswahrscheinlichkeiten als mit 2 oder 3 Eiern; die Unterschiede sind nicht signifikant (getestet nach HENSLER \& NiCHOLS 1981). 


\section{Diskussion}

\subsection{Prädationsmuster und Prädatoren an Nestern der Mönchs- grasmücke}

MOREAU (1944), LACK (1948) und SKUTCH (1949) stießen durch wegweisende Arbeiten in den vierziger Jahren des vergangenen Jahrhunderts das Fenster zu einer neuen Forschungsrichtung auf: der Analyse von Lebenszyklusmerkmalen, also der Verknüpfung von Evolution und Populationsbiologie (RICKLEFS 2000). Sie stellten fest, dass die Reproduktionsrate bei Vögeln mit verschiedenen biotischen und abiotischen Parametern in Zusammenhang steht und dass spezifische Unterschiede Ausdruck einer Adaption an diese Parameter darstellen. Lack entwickelte daraufhin das Konzept der optimalen Gelegegröße und betonte besonders den Effekt der Nahrungsverfügbarkeit, nach der die mittlere Gelegegröße evolviert. Skutch bezweifelte, dass immer ebenso viele Jungvögel aufgezogen werden, wie ernährt werden können und gab zu bedenken, dass auch andere Faktoren wie Nestprädation eine große Rolle spielen können. Er legte dar, dass eine geringere Anzahl von Jungvögeln eine verminderte Fütterrate und damit Aktivität am Nest bedeuten, was zu einer Verringerung der Prädationsrate führen könnte. Auch wenn es ausreichend Hinweise dafür gab, dass Prädation auf Lebenszyklusmerkmale wie die Gelegröße wirken kann, setzte sich Lacks Sichtweise durch und Skutchs Überlegungen blieben lange unbeachtet. So wurde die Frage, wie sich Vogelgilden oder Vogelgemeinschaften zusammengesetzt haben, bis in die 80er Jahre hinein ausschließlich unter Berücksichtigung der Verteilung von Nahrungsressourcen und Konkurrenz diskutiert (z.B. WIENS 1977, Wiens \& RottenberRy 1979, Conell 1980, RotenberRy \& Wiens 1980 , SCHOENER 1982, CONELL 1983, SCHOENER 1983, ROUGHGARDEN 1983).

Spätestens LACK (1968) und RICKLEFS (1969) nannten die Nestprädation als einen der Hauptfaktoren für die Ausbildung unterschiedlicher Reproduktionsstrategien. Ungeachtet dieser Erkenntnis ruhte die Thematik weitgehend bis zum Beginn der 80er Jahre (SLAGSVOLD 1982) und wurde erst wieder von SLAGSVOLD (1984) aufgegriffen, der nachwies, dass es gute Gründe gebe, die "nest-predation-hypothesis“ oder "Skutch-Snow-hypothesis" eingehender zu untersuchen. LIMA (1987) zeigte in einer Modellberechnung, dass für eine Optimierung der Gelegegröße die Prädation als Faktor allein ausreichend ist, nicht jedoch die Verfügbarkeit von Nahrung. Dies mag 
MARTIN (1988a) dazu bewogen haben, zunächst über die Frage nachzudenken, ob Konkurrenz als ausschließlicher Faktor für die Zusammensetzung von Vogelgemeinschaften angesehen werden kann, um dann die Frage nach der Beziehung zwischen Neststandorten und Prädation aufzugreifen (MARTIN 1988b) und schließlich auf die Beziehung zwischen Prädation und Gelegegröße einzugehen (MARTIN 1988c). Seitdem wird Nestprädation als einer der Hauptfaktoren der Evolution von Lebenszyklusmerkmalen angesehen.

Prädationsraten an Singvogelnestern reichen von 0 \% (HOOVER ET AL. 1995) bis 100 \% (MøLLER 1988). NICE (1957) kalkuliert den durchschnittlichen Bruterfolg von 7788 Nestern in 37 Untersuchungen an verschiedenen Singvogelarten auf $49 \%$, was den Ergebnissen dieser Untersuchung entspricht. Brutverluste sind nur ausnahmsweise in größerem Ausmaß anderen Faktoren wie ungünstiger Witterung oder menschlicher Einwirkung zuzuordnen (MORTON ET AL. 1993, BARKOW 2001a). Viel zitiert wird die Angabe von Best \& StAUfFeR (1980), wonach Prädation im Schnitt $80 \%$ der Nestverluste ausmacht, ein Wert, der auch in dieser Arbeit errechnet wurde. Es bestehen nicht nur gravierende Unterschiede in der Prädationsrate der Nester von Arten innerhalb eines Untersuchungsgebietes (z.B. BEST \& STAUFFER 1980, SUAREZ ET al. 1993, Martin 1993A, Yanes \& Suarez 1995, Heske et al. 1999, Davison \& BOLLINGER 2000, BARKOW ET AL. 2001, WEIDINGER 2001), sondern auch bei einer Art in verschiedenen Untersuchungsgebieten (z.B. MøLLER 1988, MARTIN \& ROPER 1988, Morton et AL. 1993, Hoover et AL. 1995, GötMARK Et AL. 1995, Vogrin \& Vogrin 1998, BARKOW 2001b). Hinzu kommen Unterschiede zwischen einzelnen Untersuchungsjahren (HATCHWELL ET AL. 1996, VogRIN \& VoGRIN 1998, BARKOW 2001b) und saisonale Schwankungen (MORTON ET AL. 1993). Generalisierbare Aussagen sind demnach nicht leicht zu treffen.

Der Nesterfolg der Mönchsgrasmücke wird in Süddeutschland mit 51,9 \% angegeben ( $\mathrm{n}=546$, BAIRLEIN ET AL. 1980). In einer anderen Arbeit nennt BAIRLEIN (1978) mit $61,5 \%$ einen höheren, STEIN (1974) mit $42 \%$ einen niedrigeren Nesterfolg. Die Werte aus den Nestfunden der Vogelwarte $(53,2 \%, n=1572)$ und der Freilandstudie (46 \%, $n=193$ ) stimmen demnach mit den Literaturdaten überein. Spezifische Vergleichswerte für die Überlebenswahrscheinlichkeit liegen nur von BARKOW ET AL. (2001) und WEIDINGER (2001) vor. Erste errechneten in einer norddeutschen Population der Mönchsgrasmücke 0,28 als Überlebenswahrscheinlichkeit für die gesamte Dauer vom Brutbeginn bis zum Ausfliegen, letzterer in Tschechien für die Brutphase 
in zwei Untersuchungsjahren 0,63 bzw. 0,47. Der Vergleich mit Untersuchungen an anderen Arten zeigt, dass verschiedene Singvogelarten tägliche Überlebenswahrscheinlichkeiten zwischen 0,89 und 0,98 haben (MAJOR ET AL. 1994, DAVISON \& Bollinger 2000, Morton et AL. 1993, Sockman 1997, Vogrin \& Vogrin 1998, WEIDINGER 2001). Für die gesamte Bebrütungsdauer des Kalifornia Mückenfänger Polioptila californica berechneten SOcKMAN (1997) 0,28, HOOVER ET AL. (1995) für Nester der Fuchsdrossel Catharus mustelinus Werte zwischen 0,12 und 1,00 und MARTIN \& ROPER (1988) für Nester der Einsiedlerdrossel C. guttatus Werte zwischen 0,07 und 0,20 . Die ermittelten Werte der Überlebenswahrscheinlichkeiten von Mönchsgrasmückennestern sind demnach weder im inter- noch im intraspezifischen Vergleich ungewöhnlich.

\subsubsection{Lokale Unterschiede der Prädationsrate}

Ende der 70er Jahre des vergangenen Jahrhunderts wurde festgestellt, dass der Bruterfolg für Nester an Übergängen zwischen zwei Habitattypen geringer sein kann als in deutlicher Entfernung von diesen Ökotonen (GATES \& GYSEL 1978). Die Autoren formulierten aus diesen Beobachtungen die sogenannte „ecological trap hypothesis" (PATON 1994), die besagt, dass vor allem Waldränder, aber auch Gehölzoder Schilfstreifen gute Lebensräume für Vögel darstellen, was insgesamt zu hohen Brutvogeldichten führt („Crowding effect“, DEBINSKI \& HOLT 2000). Hohe Siedlungsdichten führen zu einer Zunahme der Prädationsrate (z.B. KREBS 1971, DunN 1977), womit die Vögel in eine ökologische Falle tappen, wenn dann der Bruterfolg für eine Konstanthaltung der Populationen nicht ausreicht.

Zahlreiche Studien haben diese Effekt seitdem vor allem mit Kunstnestern untersucht (z.B. Yahner \& Wright 1985, Angelstam 1986, Møller 1988, Yahner \& Scott 1988, Møller 1989, YAHNeR et Al. 1989). Zwei Übersichtsartikel (PATON 1994, ANDRÉN 1995) analysieren jeweils etwa 40 Untersuchungen und kommen zu dem Schluss, dass Randeffekte der Prädation eine generelle Beobachtung sind und die "ecological trap hypothesis“ somit zutrifft. Allerdings räumen die Autoren ein, dass nicht zwangsläufig jeder Rand einem solchen Effekt unterliegt. Besonders Paton scheint der Nachweis eines allgemeinen Randeffektes wichtig gewesen zu sein, seine Metaanalyse erlaubt jedoch auch eine andere Interpretationsmöglichkeit. $\mathrm{Er}$ schreibt, dass in 10 von 14 Artikeln mit Kunstnestexperimenten und bei 4 von 7 mit echten Nestern ein Randeffekt nachgewiesen wurde, was 79 \% der Fälle bedeutet. 
Bei Betrachtung der einzelnen Experimente ergeben sich bei Kunstnestern 16 mit und 17 ohne Randeffekt, bei echten Nestern 3 mit und 5 ohne, was nur insgesamt 46 $\%$ ausmacht. LAHTI (2001) stellte die Frage, ob es gerechtfertigt sei, von einem allgemeinen Randeffekt der Prädation zu sprechen. Er fasst 54 Untersuchungen zusammen, von denen 13 bei jedem Test einen Randeffekt zeigten, 10 bei mindestens einem Test, aber 31 bei keinem einzigen. Bei einer Analyse von 13 neueren Kunstnestexperimenten kamen HARTLEY \& HUNTER (1998) zu einem vergleichbaren Ergebnis: nur 5 von 15 Tests zeigten einen Randeffekt. Eine weitere Metaanalyse von 22 Untersuchungen mit Kunstnestern zeigte aber, dass es offensichtlich doch einen Trend zu Randeffekten gibt (SÖDERSTROM 1999): wenn ein Unterschied zwischen der Nestprädation in einem Ökoton und den angrenzenden Habitaten besteht, dann in der Richtung wie von GATES \& GYSEL (1978) erwartet.

Hauptauslöser für die große Beachtung, die dem Randeffekt zuteil wurde, ist die Beobachtung, dass in den Vereinigten Staaten und Kanada zahlreiche Vogelarten, und zwar vor allem ziehende Arten, regelrechte Populationseinbrüche erfahren haben (RoBbins ET AL. 1989, Askins ET AL. 1990). Man befürchtet, dass durch Fragmentierung der Landschaft der Anteil an Rändern in der Landschaft zu Ungunsten geeigneter Flächen innerhalb der Habitate zunimmt und somit eine Landschaft im Extremfall ausschließlich aus Rändern besteht. Im Falle der Gesetzmäßigkeit eines allgemeinen Randeffektes wären fatale Folgen zu erwarten. Die Befürchtung, dass die Fragmentierung der Landschaft negativen Einfluss auf die Avifauna haben könnte, fußt auf der Arten-Areal-Beziehung, einer Hauptkomponente der Insel-Biogeografie (MACARTHUR \& WILSON 1967). Die Gültigkeit dieser Beziehung für Systeme auf dem Festland ist erwiesen (BEGON ET AL. 1991) und besteht auch für Vögel (ebd., DeBINSKI \& HOLT 2000, MARTIN 1988b). Bei zunehmender Fragmentierung der Landschaft werden einzelne Flächen immer kleiner, wodurch Habitat verloren geht. Wenn der Habitatanteil unter $50 \%$ sinkt, treten Isolation der Flächen und Randeffekte hinzu, deren Auswirkungen bei Sinken unter $20 \%$ Habitatanteil sprunghaft ansteigen (ANDRÉN 1994). Es kann zwar davon ausgegangen werden, dass besonders bei Vögeln auch kleine, isolierte Flächen immer wieder besiedelt werden können (OPDAM 1991). Da aber selbst häufige Arten Populationsrückgänge zu verzeichnen hatten (BAUER \& BERTHOLD 1997), ist es in jedem Fall gerechtfertigt, auch über mögliche interspezifische Effekte wie Räuber-Beute-Beziehungen nachzudenken.

ANDRÉN ET AL. (1985) wiesen als erste einen Effekt der Habitatfragmentierung für die 
Prädationsrate bei Kunstnestern nach. Nach ihnen haben zahlreiche Autoren, meist mit Kunstnestexperimenten (YAHNER \& PIERGALLINI 1998, YAHNER \& WRIGHT 1985, Martin 1987, Møller 1988, Santos \& Tallería 1992, Burger et AL. 1994, YahNer \& Mahan 1996, Bayne \& Hobson 1997, Darveau et AL. 1997, MattheWs et AL. 1999, Boulet \& DARVEAu 2000, SovadA ET AL. 2000, siehe außerdem HARTLEY \& HUNTER 1998 als Übersicht über 13 und PATON 1994 über 8 Untersuchungen), aber auch an echten Nestern (HoOver ET AL. 1995, Hoover \& BritTINGHAM 1998, BuRHANS \& THOMPSON 1999), die Effekte von Fragmentierung, geringen Flächengrößen und Habitatkorridoren in verschiedenen Habitaten und für unterschiedliche Vogelarten nachgewiesen. Kleinflächigkeit in der Landschaft scheint demnach für viele Arten zu erhöhter Nestprädation zu führen. Einige Autoren konnten diesen Schluss nicht nachvollziehen (HUHTA 1995, HANNON \& COTTERILL 1998, HUHTA ET AL. 1998, MATESSI \& BoglianI 1999). De GraAF (1995) konnte zwischen Nutz- und Naturwaldfragmenten keinen Unterschied feststellen und andere Autoren legten im Widerspruch zu ANDRÉN ET AL. (1985) dar, dass Prädation in natürlichen Landschaften (HALUPKA 1998a) oder größeren Waldflächen (LANGEN ET AL. 1991, HANNON \& COTTERILL 1998) sogar größer sein kann. MATESSI \& BOGLIANI (1999) fassen zusammen, dass sich Generalisierungen aufgrund von Unterschieden der historischen Gegebenheiten einer Landschaft, des Maßstabs der Fragmentierung, artspezifischer Unterschiede und der vorhandenen Prädatorengilden kaum treffen lassen.

Diese Aussage führt zu den Ursachen der unterschiedlichen Prädationsraten, sowohl bei verschiedenen Flächengrößen als auch bei Habitatgrenzen: Das Spektrum der anwesenden Prädatoren bestimmt, ob und in welcher Form ein Effekt eintritt. Schon die vermuteten Ursachen für einen Randeffekt der Prädation gehen ausschließlich vom Prädatorenverhalten aus (MARINI ET AL. 1995): 1. Die Prädatorenaktivität ist in Bereichen mit hoher Beutedichte größer; 2. Prädatoren treten häufiger an Ökotonen auf als im Waldinnern; 3. An Ökotonen gibt es mehr Prädatorenarten und 4. Prädatoren suchen entlang von Leitlinien nach Nahrung. ANDRÉN ET AL. (1985) zeigten, dass die Prädationsrate in den fragmentierten Flächen mit einer höheren Corvidendichte in Verbindung steht (s. auch HuHTA ET AL. 1996, BAYNE \& HOBSON 1997a, HOOVER \& BRITTINGHAM 1998). MøLLER (1988) führte diesen Ansatz weiter aus und wies nach, dass entlang eines Gradienten von 200 m außerhalb eines Waldstückes bis $200 \mathrm{~m}$ in den Wald hinein die Prädation auf Kunstnester durch verschiedene Rabenvogelarten unterschiedlich ist: Im freien Feld waren lediglich Aaskrähe und Elster Pica pica aktiv, 
im Waldinnern ausschließlich Eichelhäher. Am Waldrand trafen sich alle drei Arten, was zu einem hohen Prädationsdruck führte. SANTOS \& TALLERía (1992) zeigten, dass der "Crowding effect" auch für Kleinsäuger als Prädatoren in kleinen Waldstücken gilt: Viele Prädatorenarten führen zu einer insgesamt hohen Prädationsrate (DE SANTO \& WILLSON 2001). In diesem Zusammenhang lassen

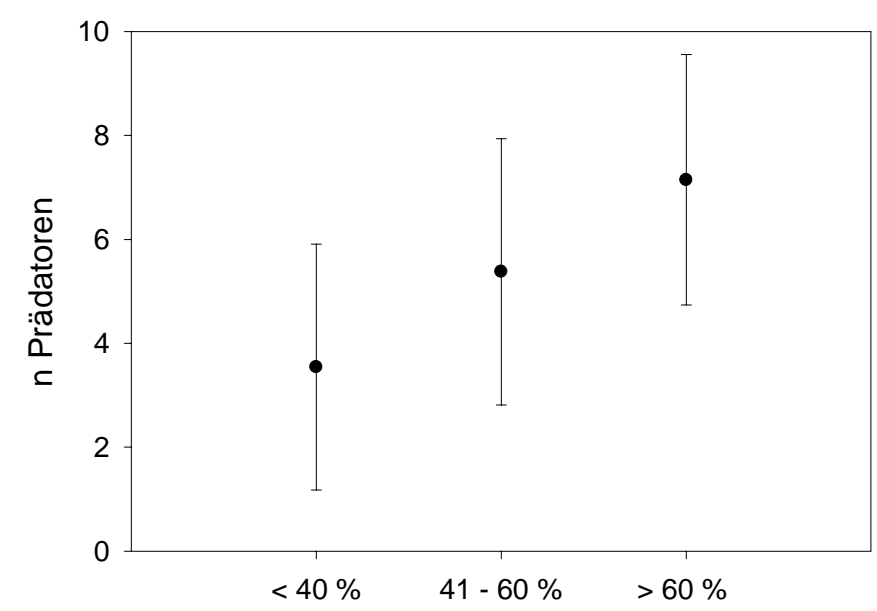
sich Daten der Übersichtsarbeit von MILLER \& KNIGHT (1993) neu berechnen, die eine Abhängigkeit der Prädationsrate von der Anzahl der Prädatorenarten belegen: In Gebieten mit hohen Prädationsraten wurden viele Prädatorenarten festgestellt (Abb. 65). Umgekehrt gilt, dass die Größe einer Fläche das Prädatorenspektrum begrenzen kann, und zwar dann, wenn die Flächenansprüche der Prädatoren unterschritten werden (MARTIN 1987, ANDRÉN 1995).

Vor dem Hintergrund dieser Abhängigkeit der Prädationsrate von der Anzahl möglicher Prädatoren lassen sich auch die Befunde der vorliegenden Arbeit erklären. Die Ergebnisse der Videoüberwachungen zeigen, dass Krähe und Elster als Prädatoren auf Mönchsgrasmückennester wenig Einfluss haben und Mäuse als Nesträuber weitgehend ausgeschlossen werden können. Es war nicht zu erwarten, dass sich ein Randeffekt, wie von MøLLER (1988) oder ein "Crowding effect" wie von SANTOS \& TALLERíA (1992) beschrieben, ergibt. Eine konkrete Erklärung für die beobachtete Reduktion der Prädationsrate bieten neben der erwähnten Begrenzung des Prädatorenspektrums durch die Flächengröße die Habitat-ansprüche des Eichelhähers, der den größten Einfluss auf die Überlebenswahrscheinlichkeit der Mönchsgrasmücke hat. Die Untergrenze für Territorien des Eichelhähers liegt bei 10 ha, offene Ackerflächen werden wegen der Gefahr durch Habichte nur ausnahmsweise überflogen (ANDRÉN 1990). Je kleiner und isolierter Waldflächen sind, desto wahrscheinlicher ist, dass der Eichelhäher nicht oder nur selten auftritt (ANDRÉN 1992). Lokale Unterschie- 
de in der Prädationsrate von Mönchsgrasmückennestern erklären sich daraus, dass der Eichelhäher hier weniger aktiv wird. Es wäre für eine endgültige Aussage erforderlich, das gesamte Prädatorenspektrum zu erfassen, was im vorgegebenen Rahmen dieser Arbeit nicht möglich war. Auch wenn SockMAN (1997) eine Überlebenswahrscheinlichkeit von 0,28 als „niedrig“ einstuft, darf eine generelle Aussage, welche Prädationsrate als „hoch“ bzw. „niedrig“ zu gelten hat, meines Erachtens nur artund ortspezifisch erfolgen und ist nur unter Beantwortung der Frage sinnvoll, ob die Verluste kompensiert werden können.

\subsubsection{Prädatoren und ihr Verhalten}

Spätestens MARTIN (1987) weist darauf hin, dass es vom Prädator oder Prädatorentyp abhängt, welche Parameter der Neststandortselektion oder Habitatwahl adaptiv sind. Diese Sichtweise wurde von zahlreichen Autoren aufgegriffen und viele Methoden sind angewandt worden, um tatsächliche Prädatoren oder das Prädatorenspektrum in einem Gebiet zu bestimmen (MAJOR 1991). Dazu gehören neben Fotofallen, wie auch in dieser Arbeit verwendet (MARTIN 1988, CARTHEW \& SLATER 1991, KUCERA \& Barrett 1993, De Graaf 1995, Danielson et Al. 1996, Hernandez et al. 1997a, Hernandez et Al. 1997b, Goetz 1981, Hensley \& Smith 1986, Picman J. 1987, Ball ET AL. 1994, Picman \& Schriml 1994, BROWder ET AL. 1995, BAYNE \& HOBSON 1997, MAJOR ET AL. 1999), Plastillineier, auf denen Schnabel- oder Zahnmuster bestimmt werden können (MølleR 1988, DARVEAU ET AL. 1997, HaNnON \& CotTERILL 1998, MATTHEWS ET AL. 1999), auch Klebefallen, an denen Haare haften bleiben (BAKER 1980, BULL ET AL. 1992) und die Bestimmung von Trittsiegeln (MARINI ET AL. 1995, NockEMANN 1998). Zur Bestimmung der Prädatorengemeinschaft und -dichte wurden Rabenvögel (ANDRÉN ET AL. 1985, MøLLER 1988, ANDRÉN 1992) und Säuger gezählt (SOVADA ET AL. 2000) oder Fotosysteme an Fallenstandorten aufgebaut (HeRnANdez et Al. 1997a, Heske et Al. 1999). Auch aus Spuren am ausgeräumten Nest wurde versucht, auf den Prädator zu schließen (MORTON ET AL. 1993, MARINI \& MELO 1998).

Als Prädatoren sind verschiedene Schlangenarten (HENSLEY \& SMITH 1986, ConRY 1988), Eidechsen (BECHET ET AL. 1998), verschiedene Zaunkönige (MAJOR ET AL. 1999), viele Rabenvögel, daneben Möven und Eulen, Waschbären, Marderartige, Hundeartige und viele Nagetiere, aber auch Herbivore und Schnecken (z.B. MILLER \& KNIGHT 1993, PATON 1994) und selbst Rotwild (PENNINGTON 1992) bekannt. 
Kaum eine der angesprochenen Methoden aber ist allein in der Lage, das gesamte Spektrum möglicher Prädatoren zu erfassen: Haarfallen können ausschließlich Säugetiere identifizieren, Fotofallen gestatten Tieren den Zugang zu Eiern, die von brütenden Vögeln hätten vertrieben werden können, durch direkte Beobachtungen treten Fehler auf, weil nachtaktive Tiere nicht feststellbar sind und bei der in der Regel kurzen Dauer eines Prädationsvorganges ist es auch bei tagaktiven Rabenvögeln fraglich, ob ein Nesträuber bemerkt wird. Bei der Beurteilung von Spuren am Nest kann nicht einmal sicher gestellt werden, welche Ursache überhaupt zum Nestverlust führte. Eine elegante Methode, die alle diese Nachteile kompensiert, ist die Verwendung von Videokameras zur Überwachung aktiver Nester (PIETZ \& GRANSFORS 1998, MCQUILLEN \& BREWER 2000), evtl. auch mit Signalübertragung durch Funk (KING ET AL. 2001).

Bei wenigen Untersuchungen wurde diese Methode bisher an Singvogelnestern angewendet und erbrachten sehr unterschiedliche Ergebnisse: THOMPSON ET AL. (1999) verzeichneten bei 25 Nestern insgesamt 11 verschiedene Prädatorenarten, die Hälfte der Nester wurden von zwei Schlangenarten prädiert; PIETZ \& GRANSFORS (2000) identifizierten an 29 ausgeräumten Nestern von wiesenbrütenden Singvögeln ebenfalls 11 verschiedene Prädatorenarten, wobei wiederum etwa die Hälfte der Verluste zwei Bodenhörnchen zuzuordnen waren; BROWN ET AL. (1998) stellten an 14 Nestern von zwei neuseeländischen Drosselverwandten Petroica australis und P. macrocephala als Prädatoren Ratten Rattus norvegicus (84 \%) und den Grunzkauz Ninox novaeseelandiae (16\%) fest; die bislang einzige mir bekannte europäische Arbeit stammt von LOPEZ-IBORRA ET AL. (2001), die einige Nester von Teich- Acrocephalus scirpaceus und Drosselrohrsängern $A$. arundinaceus filmten und Eidechsennatter Malpolon monspessulanus, Ratte Rattus sp., Ginsterkatze Geneta geneta und Kukkuck Cuculus canorus bestimmten. Alle Autoren bescheinigen der verwendeten Methode einen minimalen Einfluss auf die Untersuchungsergebnisse, was auch in der vorliegenden Arbeit bestätigt werden kann (vgl. Überlebenswahrscheinlichkeit Nestfunde 0,23 und Freilandstudie 0,24). BROWN ET AL. (1998) weisen darauf hin, dass Videoüberwachung deswegen für die Untersuchung gefährdeter Arten geeignet ist. Beeinflussungen durch die IR-Beleuchtung können ausgeschlossen werden, da Carnivore und Nagetiere zwar über Farbsehen verfügen (JACOBS 1993), aber nicht in der Lage sind, Licht mit einer Wellenlänge von mehr als $600 \mathrm{~nm}$ zu sehen. Vielmehr ist das visuelle System besonders bei Mäusen darauf ausgerichtet, im ultravioletten Be- 
reich zu differenzieren (JACOBS ET AL. 1991, JACOBS 1992).

Meine Befunde fügen sich in die bislang veröffentlichten Beobachtungen ein: Eine einzige Art oder wenige Prädatorenarten sind für die meisten Prädationen verantwortlich, im vorliegenden Fall ist es der Eichelhäher. Die anderen Nestprädationen verteilen sich auf ein breites Spektrum von hier 11 Arten (SELL 1998 filmte auf der Flächen um Schloss Möggingen neben den von mir identifizierten Prädatoren noch Dachs Meles meles, Hermelin Mustela erminea, Haselmaus und Neuntöter Lanius collurio), die wie der Steinmarder nur gelegentlich oder wie die anderen Arten vereinzelt Nester ausnahmen. Im europäischen Kontext bestätigen die Ergebnisse darüber hinaus die Untersuchungen aus Schweden (ANDRÉN ET AL. 1985, ANGELSTAM 1986, Møller 1988, Møller 1989, GötmaRK 1992, ANDRÉN 1992) und Deutschland (BARKOW ET AL. 2001, vgl. auch PATON 1994), die ebenfalls Rabenvögel als Hauptverursacher von Nestverlusten angeben. Eine Einschränkung sei hier getroffen: Bei den untersuchten Vögeln handelte es sich um gebüschbrütende Arten mit offenen Nestern wie Drosseln und Grasmücken. Die Verhältnisse für geschlossene Nester (MøLLER 1989) sowie für die von Wiesenbrütern mögen deutlich verschieden sein (Bellebaum \& EikHORSt 2000, Bellebaum 2001).

Die Beobachtung, dass Eier nach und nach verschwinden können, ist vielen Ornithologen geläufig. So versah ein Mitarbeiter der Vogelwarte ein Drosselei mit einer Nummer und stellte am nächsten Tag fest, dass ein anderes Ei im Nest lag, ein Vorgang der sich einige Male wiederholte (FIEDLER, mündl.). Die Daten der vorliegenden Untersuchung erklären diese Beobachtungen: Eichelhäher nehmen nicht die gesamte Brut auf einmal mit, sondern kommen mehrmals zum Nest, wenn man von einer geringen Wahrscheinlichkeit dafür ausgehen kann, dass ein Nest am gleichen Tag zwei- oder dreimal von verschiedenen Eichelhähern gefunden wird. Offensichtlich müssen Eier einzeln transportiert werden, denn auch wenn Eichelhäher bis zu zwölf Eicheln im Kropf transportieren können, würden Eier zu mehreren vermutlich zerbrechen. Voraussetzung hierfür ist, dass sie den Neststandort wiederfinden. Rabenvögel verfügen über ein gutes räumliches Erinnerungsvermögen (SHETTLEWORTH 1990, KREBS 1990). Es konnte auch nachgewiesen werden, wie sich Rabenvögel das Muster ihrer Umwelt einprägen, indem sie sich auffällige Landmarken merken (BENNETT 1993). Nun ist das Verstecken und Wiederfinden von Futter nicht dasselbe wie Nester fremder Vögel auf- und wiederzufinden. Aber SONERUD \& FJELD (1987) konnten in einem Experiment zeigen, dass sich zumindest Aaskrähen auf 
Kunstnester trainieren lassen und diese auch im Folgejahr eher finden als neu hinzugekommene. Die Beobachtungen der vorliegenden Arbeit weisen darauf hin, dass Eichelhäher in der Lage sind, sich auch die Standorte echter Nester zu merken und dass "Lernen“ nicht erforderlich ist, sondern ein einzelner Besuch ausreicht, um ein Nest wiederzufinden. Während Eichelhäher das ganze Jahr über pflanzliche Nahrung fressen, ist der Proteinbedarf während der Brut und Jungenaufzucht deutlich erhöht und es werden, wie bei vielen anderen Singvögeln auch, vor allem tierische Bestandteile verfüttert (GLUTZ VON BLOTZHEIM \& BAUER 1993). Eier und Jungvögel sind normaler Nahrungsbestandteil und eine verlässliche Proteinquelle, wenn die Eichelhäherjungen im Mai schlüpfen (BEZZEL 1993). Hiermit ergibt sich eine Erklärung für die Abnahme der Prädationsrate in Laufe der Saison: wenn die jungen Eichelhäher Mitte Juni ausfliegen, werden Eier und Jungvögel als Nahrung weniger dringend erforderlich. Mit diesen Beobachtungen würde ebenfalls erklärt, wieso die Prädationsrate dichteabhängig ist: Im Juli sind die Nester anderer Singvögel weniger notwendig, aber auch schwerer zu finden. Die Eichelhäher stellen dann die systematische Suche nach und nach ein.

Alle anderen Prädatoren scheinen Mönchsgrasmückennester nur hin und wieder zu finden. Eine systematische Suche nach Nestern durch diese Arten erscheint auch nicht plausibel, da selbst mehrere Nestinhalte nur einen geringen Anteil des Nahrungsbedarfes eines Marders, Fuchses oder Dachses decken können. DuNN (1977) zeigte, dass selbst Mauswiesel nur unter besonderen Bedingungen von ihrer Vorzugsbeute (Mäuse) abweichen und Meisennistkästen aufsuchen.

\subsection{Reduktion der Prädationsrate}

\subsubsection{Heimliches Verhalten, Verteidigung und Reduktion der Gelegegröße}

Nestprädation steigt mit der Aktivität der Eltern am Nest (MARTIN ET AL. 2001). Es ist daher zu erwarten, dass Altvögel sich in Nestnähe so unauffällig wie möglich verhalten: Nester mit Eiern werden im Regelfall besetzt angetroffen. Sie verlassen, wie unter 4.2.1 beschrieben, das Nest erst, wenn man sich bis auf einen Meter näherte oder sogar erst, wenn man die Hand zum Nest streckte, um die Eier zu kontrollieren. Dieses Verhalten kann an verschiedenen anderen Singvögeln wie z.B. Sindrossel und Amsel in gleicher Weise beobachtet werden. Die Altvögel bleiben auf dem Nest, bis es unzweifelhaft entdeckt und die Gefahr für sie selber zu groß wird. Eine Paral- 
lele bieten Singdrosseln, bei denen Neststandortselektion einen Kompromiss zwischen Deckung und Aussicht auf die Umgebung darstellt. Versteckte Kunstnester werden zwar seltener gefunden, die Singdrossel profitiert aber davon, nahende Feinde früh zu sehen (GöTMARK ET AL. 1995).

Nestverteidigung kann einen merklichen Einfluss auf den Bruterfolg haben (MCLEAN \& RHOdes 1991, Berg 1996, Cresswell 1997a). Allerdings nehmen Altvögel das Risiko selber zur Beute zu werden nur dann in Kauf, wenn Angriffe auf den Nestfeind Erfolg versprechen (WINKLER 1987, MCLEAN \& RHODES 1991). Aus den vorliegenden Ergebnissen lässt sich demnach schließen, dass für Mönchsgrasmücken Verteidigung gegen andere Tiere als Mäuse mit zu hohem Risiko für die Altvögel verbunden wäre: Raubsäuger würden ebensogut einen Altvogel erlegen wie die Brut. Die Verteidigung gegen Mäuse scheint dagegen effektiv zu sein, zumindest so lange, wie die Mäuse noch nicht direkt am Nest fressen und ein Überraschungseffekt eintreten kann. Die Frage, inwieweit die Risikobereitschaft mit zunehmendem Brutstatus oder Alter der Jungen erhöht ist (ONNEBRINK \& CURIO 1991, BuRES \& PAVEL 1998, GHALAMBOR \& MARTIN 2000, BuRES \& PAVEL 1997), konnte aufgrund der geringen Anzahl an Beobachtungen nicht bearbeitet werden.

Echtmäuse Muridae sind in der Lage Eier und Jungvögel zu fressen. Die Ergebnisse zeigen aber, dass sie als bedeutende Prädatorengruppe ausgeschlossen werden können. Das unterschiedliche Auftreten von Prädatoren an videoüberwachten $\mathrm{Ne}$ stern und Fotofallennestern zeigt, dass Mönchsgrasmücken Mäuse von den Nestern weitgehend fernhalten können. Dafür sprechen auch die Beobachtungen von einzelnen Mäusen, die in Nestnähe auftraten und die Vögel dennoch nicht belästigten. An einem überwachten Nest eines Zilpzalp konnte beobachtet werden, dass das Nest erfolgreich verteidigt wurde (SCHAEFER in Vorber.). Andere Videountersuchungen belegen, dass Mäuse nur vereinzelt als Prädatoren auftreten (THOMPSON ET AL. 1999, Pietz \& Gransfors 2000), Morton ET AL. (1993) fanden an 1132 Nestern der Dachsammer Zonotricha leucophrys keinen Fall von Prädation durch Mäuse. MAXsON \& ORING (1978) wiesen als erste darauf hin, dass vor dem Brutbeginn Echtmäuse und seltener Wühlmäuse als Nestprädatoren in Frage kommen. Nach dem Brutbeginn waren die Altvögel des untersuchten Drosseluferläufers Actitis macularia in der Lage, Mäuse zu vertreiben. Einen anderen Befund dokumentiert BURES (1997), der in Jahren mit hohen Populationsdichten der Feldmaus Microtus arvalis hohe Prädationsraten an Nestern des Wiesenpiepers durch diese Art nachweist. Bei der Mönchsgras- 
mücke konnte keine Prädation durch Wühlmäuse nachgewiesen werden, vermutlich weil sie die Eierschalen nicht aufbeißen können: die Eier in zwei Nestern, an denen Rötelmäuse fotografiert wurden, blieben unbeschädigt.

Wie bereits angesprochen, besteht seit langem Uneinigkeit darüber, wie die Ergebnisse von Kunstnestexperimenten mit und ohne Fotofallen zur Bestimmung der Prädatoren zu bewerten sind. Besonders im Hinblick auf landschaftliche Aspekte und Neststandorte wurden solche Ansätze vielfach angewendet, vor allem weil die Methode einfach ist und schnell zu Ergebnissen führt (MAJOR \& KENDAL 1996). Nicht nur von diesen Autoren ist mehrfach auf die Probleme dieser Methode hingewiesen worden und vor allem darauf, dass die Prädationsraten an Kunstnestern und echten $\mathrm{Ne}$ stern divergieren. Bei Arbeiten, die sich auf Singvogelnester bezogen, wurden als Ködereier meist Wachteleier verwendet, die erheblich größer sind als die meisten Singvogeleier und zum Ausschluss kleinerer Nager führen können (HASKELL 1995, De GraAf et AL. 1999, Davison \& Bollinger 2000). Vergleiche zwischen der Prädationsrate an Kunst- und Echtnestern zeigen, dass sich Folgerungen von der einen auf die andere Methode verbieten (DAVISON \& BOLLINGER 2000, WEIDINGER 2001), so dass man sehr genau überlegen muss, welche Information man aus einem solchen Experiment ziehen darf (MARTIN 1987, ANDRÉN 1995).

Meine Untersuchungen weisen nach, dass auch mit Fotofallen an ehemaligen Neststandorten und Eiern adäquater Größe nicht unbedingt Ergebnisse erzielt werden können, die den natürlichen Gegebenheiten entsprechen. Grund hierfür ist die Fähigkeit der Altvögel, Mäuse in der Regel von den aktiven Nestern fernzuhalten. An den Fotofallennestern können sie ungehindert auftreten, auf ihren Streifzügen die Eier fressen und einen unnatürlichen „Prädationsdruck“ erzeugen. Das Fehlen gröBerer Säugetiere ist vermutlich entweder ein methodischer Artefakt, d.h. die Tiere meiden fremde Objekte, oder die Mäuse sind, im Verbund mit dem Eichelhäher, einfach schneller und ein Nest ist schon ausgeräumt, bis ein Marder vorbeistreift. Ich würde mich gemäß diesen Ausführungen den letztgenannten Autoren anschließen und dazu anmerken, dass Kunstnester und Fotofallen interessante Hinweise liefern können, aber ohne eine methodische Eichung wie z.B. durch Videoaufnahmen nicht interpretierbar sind.

Die Beobachtung, dass die Überlebenswahrscheinlichkeit vor dem Schlupf geringer ist als danach, lässt Rückschlüsse auf das Prädatorenverhalten zu: Sie finden die 
Nester vermutlich nicht in erster Linie durch Beobachten der Altvögel, denn das sollte nach dem Schlupf erheblich leichter sein und zu mindestens gleich hohen Verlusten führen, eher durchsuchen sie wohl systematisch geeignete Strukturen (auch SOCKMAN 1997, VOGRIN \& VOGRIN 1998). Wenn die Überlebenswahrscheinlichkeit nach dem Schlupf geringer ist, ließ sich im Gegensatz dazu nachweisen, dass es im Untersuchungsgebiet Prädatoren gibt, die Nester durch Beobachten der Altvögel beim Nestbau und Füttern finden (MORTON ET AL. 1993). Die Prädationsrate nimmt mit der Nesterdichte zu, was sowohl im Vergleich verschiedener Flächen als auch im saisonalen Verlauf beobachtet wurde (KREBS 1971, GÖRANSSON ET AL. 1975, DUNN 1977, Martin 1988A, Morton et Al. 1993, BAyne \& Hobson 1997a). Besonders Rabenvögel suchen bei hoher Nesterdichte aktiver nach Nestern (MAJOR ET AL. 1994). Auch die Prädation an Nestern der Mönchsgrasmücke scheint saisonal dichteabhängig zu sein. Bei der Berechnung wurden Nester im April und Ende Juli nicht einbezogen, weil das Legemuster zeigt, dass die spät im April angelegten Nester bereits zur Hauptphase der Brutsaison gehören. Die geringe Stichprobe resultiert lediglich aus der Begrenzung zwischen Ende April und Anfang Mai, die Stichprobe in der zweiten Julihälfte ist zu gering.

Ausgehend von diesen Ergebnissen, war nicht zu erwarten, dass die Prädationsrate von der Gelegegröße abhängt, wie von SKUTCH (1949) vermutet und mittlerweile auch nachgewiesen wurde (MARTIN ET AL. 2001). Für eine Selektion geringerer Gelegegrößen, um die Aktivität an Nestern zu vermindern, sie unauffälliger zu machen und dadurch die Prädationsrate zu minimieren, gibt es keine Anzeichen.

Fazit: Die Mönchsgrasmücke kann Prädation durch Mäuse weitgehend ausschließen und zeigt in Nestnähe ein sehr unauffälliges, heimliches Verhalten.

\subsubsection{Neststandortselektion bei der Mönchsgrasmücke}

Verschiedene Faktoren wirken auf die Neststandortselektion von Singvögeln. (SCHAEFER 1976). Eine generelle Einschränkung für die Wahl des Neststandortes stellt die Morphologie der jeweiligen Vogelart dar (LEISLER 1981, LEISLER \& WINKLER 1991). Schlüpfende Arten können wesentlich verstecktere Stellen in der Vegetation erreichen und dort Nester anlegen (BAIRLEIN ET AL. 1980, SCHULZE-HAGEN ET AL. 1996). Weitere Faktoren sind neben der Verfügbarkeit (HANSELL 2000) und Transportmöglichkeit von Nistmaterial (GÖTMARK ET AL. 1995) physiologischer Natur: Die Notwendigkeit, eine möglichst optimale Inkubationstemparatur zu erreichen (ebd.), 
führt zu zahlreichen Anpassungen hinsichtlich der Nestanlage (COLLIAS \& COLLIAS 1984, Walsberg 1985, Yanes et Al. 1996. Außerdem muss Nässe im Nest vermieden werden (MARTIN \& ROPER 1988, HALUPKA 1998b) und sie müssen gegen Wind geschützt sein. KNOPF \& SEDGWICK (1992) untersuchten 34 verschiedene Neststandortparametern des Goldwaldsängers Dendroica petechia und fanden keine Beziehung zwischen Parametern und Prädation: Wichtig war nur die Dicke des tragenden Astes.

HANSELL (2000) betont, dass „es zahlreiche und detaillierte Hinweise des Einflusses der Prädation auf die Wahl des Neststandortes im Vergleich zu physiologischen Faktoren oder der Verfügbarkeit von Nistmaterial gibt, so dass sie [die Prädation] das stärkste Argument dafür ist, dass das Überleben der Brut zum Ausfliegen vom Neststandort abhängt“" (s. auch NILSSON 1984, MARTIN \& ROPER 1988, MARTIN 1993a+b).

So wurden Unterschiede zwischen Prädationsraten und verschiedenen Nesthöhen vielfach nachgewiesen. Danach können niedrigere Nester oder Bodennester einen geringeren Bruterfolg haben als höher gelegene (BEST \& STAUFFER 1980, MARTIN 1988C, MARTIN 1993B, BAYNe ET AL. 1997, WILSON \& COOPER 1998). Dagegen stellten Bairlein et al. (1980), YAHNer \& Cyper (1987), Martin (1988d) und HalupKa (1998b) den umgekehrten Effekt fest. Halupka zeigt, dass die von inm untersuchten Wiesenpieper (Anthus pratensis) niedrige Neststandorte unter Grasbulten nur im trockenen Zustand wählen. Auf den Bulten sind die Nester weniger gut versteckt und eher Opfer von Prädatoren. BAIRLEIN ET AL. (1980) vergleichen vier Grasmückenarten hinsichtlich ihrer Neststandorte und ihrem Bruterfolg und fanden, dass die Dorngrasmücke (S. communis) die niedrigsten Neststandorte bevorzugt und die höchsten Nesterfolge aufweist. Diese Beispiele illustrieren, was MARTIN (1993b) darstellt: Die lange Zeit gültige Annahme, dass die Prädationsrate der Nester von Bodenbrütern generell höher ist als der Nester von Gebüschbrütern, lässt sich nicht bestätigen. Er fand eine differenziertere Beziehung, nach der im Wald Boden- und Kronendachbrüter sicherer sind als Gebüschbrüter und dass es in offenen Landschaften eher umgekehrt ist.

Das Beispiel des Wiesenpiepers und weitere Beobachtungen von BEST \& STAUFFER (1980) sowie WILSON \& COOPER (1998) zeigen, dass nicht unbedingt die Höhe allein eine Rolle spielen muss, sondern auch die Sichtbarkeit eines Nestes, die sich mit der Nesthöhe ändert. MARTIN (1992) legt dar, dass in zahlreichen untersuchten 
Kunstnest-experimenten solche Nester mit höherer Deckung eine geringere Prädationsrate erfuhren. Andere Autoren bestätigen diese Befunde für echte Singvogelnester (z.B. MARTIN \& ROPER 1988, HATCHWELl ET AL. 1996, SOCKMAN 1997). Allerdings sind auch hier die Befunde nicht so eindeutig, wie es zunächst schien. GÖTMARK ET AL. (1995) bescheinigen MARTIN \& ROPER (1988) einen methodischen Einfluss, da dieser nur Kunstnester betrachtet hatte und es an echten Nestern seltener einen Unterschied zwischen der Prädationsrate an versteckten und weniger versteckten Standorten gibt (FILLIATER ET AL. 1994, GÖtMARK ET AL. 1995, BuRHANS \& THOMPSON 1998, VogRIN \& VogRIN 1998, HOOVER \& BRITTINGHAM 1998). In einem experimentellen Ansatz zeigten HowletT \& STUTChBuRY (1996) sogar, dass das Offenlegen von Nestern ohne Einfluss auf die Prädation blieb.

Für die Neststandortwahl der Mönchsgrasmücke liegen zahlreiche Untersuchungen vor: BAIRLEIN ET AL. (1980) geben an, dass die Nester der Mönchsgrasmücke zu etwa $25 \%$ in Nadelgehölzen angelegt werden, weniger als $7 \%$ in Kräutern und die restlichen in Laubgehölzen. BAIRLEIN (1978) fand im Park von Schloss Möggingen etwa 40 \% der Nester im Europäischen Pfeifenstrauch Philadelphus coronarius, im Liguster Ligustrum vulgare oder im Holunder Sambucus niger und verweist, dass diese Verteilung der relativen Häufigkeit dieser Arten entspricht. Das gleiche gilt für Auwälder in der Umgebung der Vogelwarte, in denen vor allem Traubenkirschen Prunus padus genutzt werden, die dort zu den wenigen zur Nestanlage geeigneten Strukturen zählen. Auch MASON (1976) nimmt für die hohe Anzahl (40\%) der in Brombeeren Rubus sp. gefundenen Nester keine Selektivität an, sondern eine Nutzung entsprechend dem Vorkommen einer Pflanze. Insgesamt werden über 100 verschiedene Pflanzenarten zur Nestanlage genutzt, was unterstreicht, dass die Mönchsgrasmükke sehr flexibel in der Wahl der Nistpflanze ist (BERTHOLD ET AL. 1990). Betrachtet man die Wahl des Neststandortes in Südfrankreich oder Finnland, wird deutlich, dass die Mönchsgrasmücke alle Strukturen nutzt, die nach ihren Auswahlkriterien geeignet erscheinen. Diese Kriterien sind offensichtlich so weit gefächert, dass viele Pflanzen in Frage kommen. BERTHOLD (1978) weist auf diesen Aspekt eingehend hin und legt dar, dass Habitate besiedelt werden, sobald eine zur Nestanlage geeignete Struktur vorhanden ist. Die Unterschiede der Prädationrate der Nester in selten und häufig genutzten Pflanzen zeigen aber, dass die Wahl nicht beliebig ist: Die Mönchsgrasmücke brütet häufiger in Pflanzen, die bessere Überlebenschanchen bieten.

Die Kriterien für die Auswahl der Neststandorte sind nicht leicht zu definieren. Ein 
Kriterium scheint optischer Schutz zu sein. Das zeigt die Nutzung von immergrünen Pflanzen in der frühen Brutsaison, wenn Bäume und Sträucher noch nicht voll belaubt sind. Im gleichen Kontext steht die Zunahme der Nesthöhe im Verlauf der Saison, auf die auch BERTHOLD ET AL. 1990 hinweisen. Niedrige Strukturen wie vor allem Brennnesseln werden weniger genutzt und die Nester auch innerhalb dieser Strukturen höher angelegt, da die Triebe im Verlauf der Vegetationsperiode hochwachsen und unten keinen Sichtschutz mehr bieten. Generell scheint die Nesthöhe vom Nestträger abzuhängen. Sie schwankt stark zwischen einzelnen Regionen. In Süddeutschland wurde eine mittlere Nesthöhe von $104 \mathrm{~cm}$ festgestellt, die niedrigsten Nester berührten fast den Boden, die höchsten waren in $650 \mathrm{~cm}$ Höhe angebracht (BAIRLEIN ET AL. 1980). Südfranzösische Nester hatten eine mittlere Höhe von $90 \mathrm{~cm}$ (ebd.), Nester in Großbritannien von $70 \mathrm{~cm}$ (MASON 1976), in Finnland wurde eine mittlere Nesthöhe von $60 \mathrm{~cm}$ ermittelt (HAARTMAN 1969). Während in Großbritannien häufig Brombeeren genutzt werden, sind es in Finnland Farne, die zu niedrigeren Mittelwerten führen. Auch innerhalb einer Region kann die Nesthöhe schwanken. So fand BAIRLEIN (1978) eine mittlere Nesthöhe von $90 \mathrm{~cm}$ in den Laubwäldern um Schloss Möggingen (v.a. Pfeifenstrauch, Liguster und Holunder), in Auwäldern der Umgebung wurden im Schnitt $127 \mathrm{~cm}$ gemessen (v.a. Traubenkirsche).

In diesen Rahmen sind meine Untersuchungsergebnisse einzuordnen. Die durchschnittliche Nesthöhe war mit $75 \mathrm{~cm}$ niedriger als von BAIRLEIN (1980) und BERTHOLD ET AL. (1990) für Süddeutschland angegeben. Bei der Nestersuche für diese Untersuchung wurden aber weder alle Nester gesucht wie bei BAIRLEIN (1978), noch wurden ganze Flächen systematisch abgesucht, wie bei der Nestersuche an der Vogelwarte üblich. Es wurden einzelne Gebiete ausgewählt und gezielt auch Brennnesseln durchsucht, um Material für Neststandortvergleiche zu erhalten. So spiegeln die gefundenen Differenzen Unterschiede in der Fragestellung wider. In Verbindung mit Daten von den Kanarischen Inseln (148 cm, BAIRLEIN ET AL. 1980), Madeira (197 +/$76 \mathrm{~cm}, \mathrm{n}=85$, QUERNER unveröff.), Italien (127 $+/-58, \mathrm{n}=22$, FIEDLER unveröff.) und Mallorca ergibt sich ein latitudinaler Gradient der Nesthöhe (auch BAIRLEIN ET AL. 1980), der zu überprüfen wäre, da z.B. die Daten von Inseln nicht mit Festlanddaten vergleichbar sind: Unterschiede könnten in der Neststandortwahl zwischen dem Festland und Inseln bestehen, auf denen keine Rabenvögel vorkommen (s.u.). Weitere Vergleichsdaten für den Mittelmeerraum liegen nur aus Kampanien, Italien, vor, wo Nesthöhen zwischen 50 und $250 \mathrm{~cm}$ (SCEBBA 1993) und Sizilien, wo 70 bis 250 
cm (ILPICHINO \& MASSA 1989) gemessen wurden; genauere Angaben werden nicht gemacht. Auf Basis dieser Daten lässt sich weder die Hypothese der latitudinalen Abnahme noch die einer möglichen Insel-Festland-Beziehung überprüfen.

Die durchschnittliche Höhe der Nistpflanze entspricht den von BAIRLEIN (1978) gemessenen Werten. Er errechnete, dass die Nester etwa in der Mitte einer Niststruktur angelegt werden, wobei sein Wert mit 53 \% etwas höher ist als der in dieser Arbeit errechnete. Für Durchmesser von Nistpflanzen und Entfernung zum Rand der Pflanze liegen keine Vergleichsdaten vor. Angesichts der vorstehenden Erörterungen kann jedoch davon ausgegangen werden, dass die Daten repräsentativ für die Mönchsgrasmücke in der Region sind.

Die Notwendigkeit für süddeutsche Mönchsgrasmücken, gut versteckte Nester anzulegen, spiegelt sich auch in den Schätzwerten für die Sichtbarkeit wider. Meine Ergebnisse zeigen, dass offene, gut sichtbare Nester eine Ausnahme darstellen. Es scheint wohl schwer zu sein, ein Nest aus allen Richtungen versteckt anzulegen, aber die meisten Nester sind aus drei Richtungen kaum zu sehen. Die einzigen Vergleichsdaten zur Deckung/Sichtbarkeit eines Nestes stammen von WEIDINGER (2001), der in Tschechien die Mönchsgrasmücke in einem Deckungsscore von 0 bis 4 als „2" einordnet. Im Vergleich mit der Goldammer, die den Wert „3“, und Singdrossel, die den Wert „1“ erhielten, kann ich diese Einordnung nachvollziehen. Die Ergebnisse der vorliegenden Arbeit bestätigen die Einschätzung von BERTHOLD (mündl.), wonach Mönchsgrasmückennester gut versteckt und nur dann sicher zu finden sind, wenn man von unten her aus dem Inneren die Vegetation durchsucht, weil sich die Nester dann als dunkle Strukturen abheben. Auch die Sichtbarkeit der Nester von Mallorca entsprechen den Erfahrungen der Mitarbeiter der Vogelwarte für Standorte auf Inseln: Auf Madeira z.B. sind die Nester manchmal „sehr offen“, „von weitem gut zu sehen“ oder auch „kaum versteckt“ angebracht (BERTHOLD, COPPACK, FIEDLER, QUERNER, mündl.). Quantifizierende Vergleichsdaten liegen allerdings nicht vor.

WILSON \& COOPER (1998) finden beim Buchentyrann Empidonax virescens keine Beziehung zwischen Neststandortparametern und Prädationsraten und vermuten, dass die Neststandortwahl zufällig ist. SCHMIDT \& WHELAN (1999) widersprechen dieser Vermutung und führen aus, dass Zufälligkeit nicht mit Vorhersagbarkeit gleichzusetzten ist. Dieses Beispiel illustriert die Vermutung von FILLIATER ET AL. (1994), die zusammenfassen, dass „eine reiche Gilde von Nestprädatoren die Existenz voraus- 
sagbar sicherer Neststandorte verhindert [...] ein sicherer Neststandort im Hinblick auf einen, könnte ein Nest empfindlicher für einen anderen Prädator machen“, HOOVER \& BRITTINGHAM (1998) führen aus, dass kein Einfluss zu erwarten ist, wenn viele Prädatoren vorhanden sind und/oder Spezialisten fehlen. Wenn es so ist, dass verschiedene Prädatorengruppen unterschiedliche Neststandorte selektieren, wäre dieser Effekt mit den bisherigen Methoden nicht darzustellen und es muss daher zu der Aussage kommen, dass die Prädation ein zufälliges Ereignis ist (auch HowLETT \& STUTCHBURY 1996). Bei der Mönchsgrasmücke konnte keine einfache Beziehung zwischen den in dieser Arbeit gemessenen Neststandortparametern und dem Bruterfolg aufgezeigt werden. Die Literatur bietet hier neben methodischen Aspekten nur eine Erklärung: Es hängt von den Prädatoren ab, welche Parameter einen Einfluss haben. Verschiedene Autoren haben gezeigt, dass Prädatorengruppen unterschiedliche Neststandorte aufsuchen: Säugetiere finden eher niedrige Nester, Vögel eher hohe (Nour et Al. 1993, LAtTA et Al. 1995, HanNon \& COTterill 1998). Best \& STAUFFER (1980) sowie SANTOS \& TALLERÍA (1992) stellen dar, dass höher angelegte Nester ein geringeres Prädationsrisiko haben, weil bodennah suchende Säuger die Nester nicht erreichen können, eine Prädatorengruppe somit wegfällt. Der Schluss, den FILLIATER ET AL. (1994) ziehen, konnte bislang nicht belegt werden, einerseits weil Daten, aber auch weil die theoretische Grundlage fehlte. SIH ET AL. (1998) füllen diese Lücke, indem sie darlegen, dass gegenläufige Selektion durch verschiedene Prädatoren auf eine Beute („multi-predator-effects“) vorhandene Adaptionen verschleiern, wenn man die Prädatoren und deren Verhalten nicht kennt. Die Autoren fordern neben Beobachtungen differenzierten Prädatorenverhaltens auch Experimente oder Untersuchungen, bei denen jeweils eine der Prädatorengruppen fehlt, um aufzuzeigen, welche Selektion jeder einzelne induziert.

Diesen Überlegungen folgend, liefern die Ergebnisse dieser Arbeit ein Beispiel für einen „multi-predator-effect“ und zeigen darüber hinaus, dass die Neststandortselektion der Mönchsgrasmücke als Strategie zur Vermeidung von Prädation zu erklären ist. Durch die Videoüberwachung in Südwestdeutschland wurde nachgewiesen, dass verschiedene Prädatorentypen unterschiedliche Neststandorte von Nestern einer Art finden. Dadurch entsteht ein Selektionsdruck aus zwei Richtungen: Säuger werden durch hohe, außen angelegte, Rabenvögel durch niedrige, zentral gelegene Nester weitgehend ausgeschlossen. Resultat sind einerseits mittlere Höhe und Position erfolgreicher Nester, die sich zwischen diesen Gruppen einordnen, andererseits aber 
auch die Variationsbreite der Neststandortwahl. Die gegenläufigen Selektionsrichtungen fördern gleichsam ein „Ausprobieren“ verschiedenster Neststandorte (FILLIATER ET AL. 1994). Dabei sind die Möglichkeiten des „Ausprobierens“ von der Morphologie der Art begrenzt: Die Mönchsgrasmücke hat nicht die Möglichkeit, durch sehr niedrige und versteckte Nester die Prädationsrate zu vermindern wie etwa die Dorngrasmücke. Ähnliche Verhältnisse finden sich beim Teich- und Sumpfrohrsänger Acrocephalus palustris: Der Teichrohrsänger hat hohe Prädationsraten und relativ auffällige Neststandorte, der Sumpfrohrsänger dagegen versteckte Standorte mit niedriger Prädation (GÄRTNER 1982, SCHULZE-HAGEN ET AL. 1996).

Leider konnte der Aspekt der Wahl des Neststandortes eines Folgenestes nicht systematisch untersucht werden. Es wurde aber bereits nachgewiesen, dass Brutplätze nach erfolgreicher Brut beibehalten oder nach einer schlechten Erfahrung gewechselt werden (MARKS 1986, MARZLUfF 1988, BolLINGER \& GAVIN 1989). Beim Trauerschnäpper hat sich sogar das Paarungssystem danach ausgerichtet, ob ein Männchen in seinem Revier prädationssichere Höhlen hat: Die Weibchen wechseln das Revier - und damit den Brutpartner, - wenn eine Brut gefressen wurde (BAUCHAU \& SEINEN 1997), was dazu führt, dass einige Männchen mehrere, örtlich voneinander getrennte Territorien verteidigen und die einmal gewonnenen Weibchen in das Ausweichrevier locken (SLAGSVOLD \& LIFJELD 1986). Vergleichbare Untersuchungen hätten eine telemetrische Markierung einiger Brutpaare der Mönchsgrasmücke gefordert, was im vorgegebenen Rahmen nicht möglich war. So bleiben einige Beobachtungen, nach denen ein Folgenest zwar nur wenige Meter vom erfolglosen Vornest entfernt lag, aber in zwei Fällen das erste Nest auf Brusthöhe in einem Busch angelegt war, das Folgenest unter einer Grasbulte versteckt lag; in einem weiteren Fall war das erste Nest in geringer Höhe in einem Holunderdickicht am Waldrand und das Folgenest in gut 3 Meter Höhe auf einem Ast angelegt.

Die Ergebnisse der Neststandorte auf Mallorca liefern den nach SIH ET AL. (1998) ausstehenden Gegenbeweis: In einem Gebiet, in dem eine Prädatorengruppe fehlt, selektiert die verbleibende eben die Neststandorte, die nach den Ergebnissen aus Südwestdeutschland zu einem weitgehenden Ausschluss führen sollten (Abb. 66). Es wäre nach dieser Untersuchung sicher falsch anzunehmen, dass der Bruterfolg mallorquinischer Nester $100 \%$ beträgt. Die Brutsaison stand am Anfang und die Dichte der Nester war zusätzlich zu der ohnehin geringeren Siedlungsdichte noch niedrig. Allerdings ist zu Beginn der Brutsaison die Prädation in SW-Deutschland am 


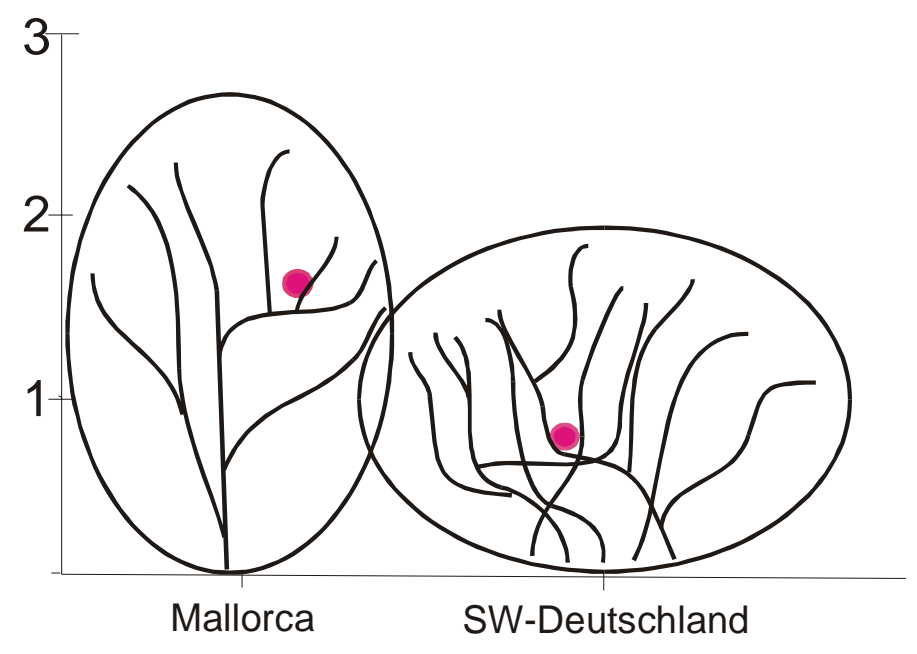

Abb. 66: Nesterstandorte der Mönchsgrasmücke auf Mallorca und in Südwestdeutschland (aus den Mittelwerten, schematisch).

höchsten, die Unterschiede zu den bekannten Siedlungsdichten für Mischwälder nicht so hoch, so dass ein Sinken der Prädationsrate auf null plausibel erscheint. Auf Madeira, wo Rabenvögel ebenfalls fehlen und die Nester vergleichbar hoch angelegt werden wie auf Mallorca, wurde in der Hauptphase der Brutsaison 1992 und 1999 eine Überlebenswahrscheinlichkeit von 0,55 festgestellt, die signifikant über der in SW-Deutschland liegt $(p<0,05$, UNVERÖFFENTL. DATEN VON BERTHOLD UND MITARBEITERN). Wie angegeben, wurden vornehmlich Vorjahresnester vermessen. Die Vegetationsperiode hatte zu diesem Zeitpunkt wegen ausbleibender Regenfälle in März und April noch nicht begonnen, die Sträucher hatten noch nicht ausgetrieben. Ein methodischer Einfluss auf die Ergebnisse ist also auszuschließen, da sich seit der Nestanlage weder die Nesthöhe noch Sichtbarkeit verändert haben konnten.

Fazit: Die Neststandortselektion der Mönchsgrasmücke ist keinesfalls zufällig, wie man aus den fehlenden Beziehungen zwischen Bruterfolg und Neststandortparametern schließen könnte, sondern steht unter dem Selektiondruck verschiedener Prädatorengruppen. Sie ist darauf ausgerichtet, den Prädationsdruck zu minimieren, was auf Inseln ohne Rabenvögel zu geringen Prädationsraten führt.

\subsection{Reduktion des Prädationseffektes}

\subsubsection{Kompensation von Brutverlusten}

Die Anzahl möglicher Brutversuche ist abhängig von der Dauer der Brutsaison. Als Beispiel sei der Vergleich zwischen Teich- und Sumpfrohrsänger angeführt (SCHULZE-HAGEN ET AL. 1996). Der Teichrohrsänger hat mit 14 Wochen eine ähnlich lange Brutsaison wie die Mönchsgrasmücke und im Schnitt einen Bruterfolg von 44,9 +/- 9,4 \%. Zweitbruten gibt es nicht, die durchschnittliche Anzahl von Nachgelegen beträgt 2,63. Die Brutsaison des Sumpfrohrsängers dauert nur 7 Wochen, Zweitbru- 
ten oder Nachgelege gibt es nicht: Der Bruterfolg liegt aber mit 68,1+/- 11,6\% deutlich höher. Ausgehend davon, dass die endogene Jahresrhythmik durch Heim- und Wegzug das Brutfenster bestimmen, könnte sich der Sumpfrohrsänger, der im südlichen Afrika überwintert, höhere Brutverluste insofern nicht „leisten“, weil sie innerhalb der vorgegebenen Zeit nicht kompensierbar wären.

Die vorliegenden Ergebnisse zeigen, dass die Mönchsgrasmücke durch schnelle Nachgelege und die Fähigkeit, viele Brutversuche durchzuführen in der Lage ist, die Gelegeverluste auszugleichen. Die Berechnung der Anzahl möglicher Brutversuche zeigt, dass auch eine Überlebenswahrscheinlichkeit von 0,24 bzw. 0,23 pro Nest ausgeglichen werden kann. Beobachtungen im Feld bestätigen diese Berechnungen, da bei der Nestersuche regelmäßig vier oder mehr Nester in einem Revier gefunden werden. BerTHOLD \& QUeRNeR (1978) konnten zeigen, dass in den Volieren bis zu sieben Brutversuche möglich sind. Für andere Arten sind ähnliche Sequenzen im Freiland belegt (FINCH 1984). Vergleichsdaten für die Dauer zwischen Verlust eines Nestes und Legebeginn eines Folgenestes sind rar, belegen aber, dass die Mönchsgrasmücke auch hier keine Ausnahme darstellt (MOUSLEY 1917, ANDERSON 1979, FINCH 1984). SCOTT ET AL. (1987) zeigten am Rotkardinal Cardinalis cardinalis und dem Katzenvogel Dumetella carolinensis, dass ein Nachgelege etwa fünf Tage nach dem Gelegeverlust begonnen wird und diese Zeitspanne für Singvögel gemäßigter Breiten normal ist. Sie bestätigen eine saisonale Abhängigkeit dieses Faktors, wie bei der Mönchsgrasmücke festgestellt, und erklären diesen Effekt durch Witterungsunterschiede: bei kaltem Wetter dauert das Nachlegen länger (auch FINCH 1984).

Die Population der Mönchsgrasmücke ist bundesweit konstant oder steigend (BAUER \& BeRTHOLd 1997, BAUER ET AL. 2002) und auch im Bodenseeraum gibt es keine Hinweise auf Bestandsveränderungen (WIDMER 1999). Aktuelle, halbquantitative Ergebnisse von Rasterkartierungen im Untersuchungsgebiet deuten auf steigende Bestände (LEY, mündl.). Ob eine gemessene Überlebenswahrscheinlichkeit der Nester als hoch oder niedrig zu bewerten ist, hängt davon ab, inwieweit die resultierende Natalität ausreicht, um die Populationen konstant zu halten, ob es sich also um Populationsquellen oder -senken handelt. Die unterschiedlichen Prädationsraten zwischen den einzelnen Untersuchungsflächen machen deutlich, dass kleine Waldstükke nicht per se negative Auswirkungen haben (TRINE 1998, MURCIA 1995). Das Fehlen einer Art ist nicht einfach mit hoher Prädation in einer solchen Fläche zu erklären (LANGEN ET AL. 1991). Doch selbst wenn Prädation als ein Faktor in Betracht kommt, 
haben Vögel durch ihr hohes Ausbreitungsvermögen die Möglichkeit auch Standorte zu besiedeln, die keine positive Reproduktionsbilanz aufweisen (OPDAM 1991). Marginale oder suboptimale Habitate werden trotz schlechter Bruterfolge immer wieder besiedelt, weil ausreichend andere Habitate mit positiver Bilanz vorhanden sind, aus denen eine Einwanderung erfolgen kann (Vogel 1998, ROtHHAUPT 1998). Verallgemeinerungen sind auch hier nicht angebracht, da dasselbe Habitat für einzelne Vogelarten günstig und für andere ungünstig sein kann (DONOVAN ET AL. 1995, BARKOW 2001b). Besonders die Ergebnisse von BARKOW (2001b) unterstützen dabei eine Überlegung von HANSSON (1989), der darstellt, dass Prädation vielfach nur in optimalen Habitaten untersucht wurde, marginale oder suboptimale Habitate für Prädatoren wichtig, für die Metapopulation aber verzichtbar sind (RоTHHAUPT 1998). Einige Ansätze in der Metapopulationstheorie weisen sogar darauf hin, dass das Gleichgewicht zwischen Populationsquellen und -senken evolutiv stabil und nicht zwangsläufig ein Zustand ist, der durch Managementmaßnahmen verändert werden müsste (MorRis 1991, Howe \& Davis 1991, Pulliam \& Danielson 1997). Es ist sehr vom Maßstab abhängig, inwieweit die lokale Situation einer Population als kritisch einzustufen ist (MURCIA 1995) und es ist deutlich, dass auch für die Mönchsgrasmücke die Betrachtung eines größeren Maßstabes erforderlich ist, um die Gefährdung der Population einzuschätzen (PULLIAM \& DANIELSON 1997).

Fazit: Nach den vorliegenden Ergebnissen ist die Mönchsgrasmücke aufgrund der langen Brutsaison und ihrer Nachlegekapazität in der Lage, Brutverluste innerhalb einer Saison und damit den Effekt der geringen Überlebenswahrscheinlichkeit auszugleichen.

\subsubsection{Brutbiologie, Gelegegröße und Überlebenswahrscheinlichkeit}

Die Ergebnisse der brutbiologischen Parameter der vorliegenden Arbeit werden von den Literaturdaten grundsätzlich bestätigt: BAIRLEIN ET AL. (1980) berechneten aus 684 süddeutschen Nestfunden als Median des Legebeginns den 15. Mai. Dieser Wert ist abhängig von der Höhe über NN und wird davon beeinflusst, in welchen Nistpflanzen die Nester gebaut wurden. Für Nester in Koniferen wird der 16. Mai, in Brennnesseln der 28. Mai und für alle anderen der 21. Mai als Median der Legebeginne errechnet. Für eine Meereshöhe von 251-500 m NN ( $n=333)$ wird als Legebeginn der 15. Mai angegeben. Der Median, der sich aus den Nestfunden ergibt, wäre demnach eine Woche später als nach der Literatur zu erwarten. Allerdings hat sich 
der Median innerhalb der Nestfunde seit 1981 nicht verändert. Der Unterschied ist unter Umständen mit den dargestellten Abhängigkeiten des Medians der Legebeginne zu erklären, weil sich die Auwälder, in denen Nester gefunden wurden, mit ca. 400 m über NN im oberen Bereich dieser Spanne befinden und Hauptnistpflanzen dort Traubenkirschen sind, bei denen der Median nach BAIRLEIN ET AL. (1980) der 21. Mai beträgt.

Der früheste Legebeginn, den BAIRLEIN ET AL. (1980) nennen, ist der 17. April. Aus abgebildeten Legemustern geht hervor, dass Nester vor dem 20. April die Ausnahme darstellten. Der aus den Nestfunden ermittelte Legebeginn am 7. April 1994 kann auch als Ausnahme gewertet werden, weil der früheste darauf folgende der 14. April (1989) war und die kontinuierliche Verteilung mit dem 20. April beginnt. Zwischen 1947 und 1989 wurde kein Legebeginn vor dem 17. April festgestellt, die Daten des frühesten Legebeginns schwanken in diesem Zeitabschnitt zwischen 17. April und 13. Mai, was darauf hindeutet, dass erst seit wenigen Jahren schon vor Mitte April die Bedingungen für eine erfolgreiche Brut vorhanden sind. Die Daten belegen langjährige Beobachtungen von Mitarbeitern der Vogelwarte, nach denen die Mönchsgrasmücke heute früher brütet als vor 20 oder 30 Jahren und führen zu der Anahme, dass sie nach Veränderungen im Zugverhalten (BERTHOLD 1990, GATTER 1992, Bezzel \& Jenz 1995, BeRTHOLd 1998, PuLIDO 2000) auch Veränderungen in der Brutbiologie zeigt, die sich mit der Klimaerwärmung in Verbindung bringen lassen (Orginaldaten der Vogelwarte Radolfzell, SCHAEFER ET AL. in Vorber.). Die Brutsaison beginnt mittlerweile zur gleichen Zeit wie in Südfrankreich (LABITTE 1955) oder Südengland (MASON 1976), wobei sich auch in diesen Gebieten inzwischen Veränderungen ergeben haben (CRICK ET AL. 1997, CRICK \& SPARKS 1999).

Veränderungen zeigen sich nicht nur hinsichtlich der Brutperiode, sondern auch mit Blick auf die Gelegegröße. BAIRLEIN ET AL. (1980) geben für süddeutsche Mönchsgrasmücken eine mittlere Gelegegröße von 4,76 Eiern pro Nest an, was deutlich über den von mir ermittelten Werten liegt. Auch bei diesem Parameter werden Unterschiede durch Meereshöhe und Bruthabitate bedingt: Nester in niedrig gelegenen Regionen haben mehr Eier, Nester in Auwäldern größere Vollgelege als in anderen Lebensräumen. Dazu kommt der durch diese Untersuchung bestätigte Kalendereffekt der Gelegegröße, der bei Vergleichen zwischen Gruppen von Nestern generell berücksichtigt werden sollte. Die vorliegenden Ergebnisse sind mit denen von BAIRLEIN (1978) vergleichbar, da seine Untersuchungsgebiete 1974 - 1976 z.T. die- 
selben waren. Er stellte bei 58 Bruten eine Gelegegröße von 4,52 fest, die mit dem ermittelten Wert aus den Nestfunden übereinstimmt. Die Gelegegröße aus der Freilandstudie, also 25 Jahre später, ist mit 4,39 deutlich geringer. Weitere Auswertungen der Nestfunde zeigten, dass die Gelegegröße über den angegebenen Zeitraum signifikant abgenommen hat. Da die Dezimalen das Verhältnis zwischen 4er und 5er Gelegen ausdrücken, würde das bedeuten, dass im Vergleich zu den Ergebnissen von BAIRLEIN in den 90er Jahren mindestens $10 \%$ der Population ein Ei weniger gelegt haben, die Abnahme innerhalb der Nestfunde belegt noch höhere Werte (Orginaldaten der Vogelwarte Radolfzell, SCHAEFER ET AL. in Vorber.). Diese Beobachtung ist neu. Bislang wurden bei Singvögeln Steigerungen der Gelegegröße festgestellt, die mit früherem Brüten zusammenhängen (WINKEL 1997, METZLER 2001). Letztgenannter zeigt eine Zunahme der Gelegegröße bei Drossel- und Teichrohrsänger bei gleichzeitiger Vorverlagerung der Brutperiode. Die vorliegenden Daten der Mönchsgrasmücke zeigen aber nicht nur eine Veränderung der Gelegegröße, sondern auch im Verlauf des Kalendereffektes (vgl. Abb. 60 und Abb. 61): Ein Anstieg der Gelegegröße von Mitte April bis Anfang Mai ist nicht mehr nachweisbar. Die Mönchsgrasmücke zeigt demnach heute einen Kalendereffekt, der für südenglische Vögel vor 25 Jahren typisch war (MASON 1976, vgl. aber CRICK ET AL. 1993, S. 89: CS-increase für die Mönchsgrasmücke: 1,08).

Die Ergebnisse der vorliegenden Arbeit und die vorangehende Darstellung bieten demnach vier Themenkomplexe zur Diskussion einer Risikoverteilung an:

1. Ausweitung der Brutsaison bei Reduktion der Gelegegröße (SCHAEFER ET AL. in Vorber.).

2. Zusammenhang zwischen Überlebenswahrscheinlichkeit und Brutbeginn im Folgejahr (Abb. 62, Abb. 63, Tab. 21, Tab. 22).

3. Zusammenhang zwischen Überlebenswahrscheinlichkeit und Gelegegröße auf einer Untersuchungsfläche (Tab. 23).

4. Zusammenhang zwischen Überlebenswahrscheinlichkeit und Gelegegröße im Folgejahr (Abb. 64, Tab. 24, Tab. 25).

Zu 1.: BERTHOLD (1990) wies als erster auf den Zusammenhang zwischen Veränderungen im Zugverhalten und Klimaerwärmung hin. Seitdem sind früherer Heim- und späterer Wegzug vielfach in Europa und auch in Nordamerika belegt worden (GATTER 1992, BuRTON 1995, WiLsON ET AL. 1998). Schon zuvor waren Ausdehnun- 
gen des Brutareals für einzelne Arten dokumentiert worden und mittlerweile hat die Hälfte der mitteleuropäischen Brutvogelarten ihr Brutareal nach Norden oder Nordwesten ausgedehnt (BURTON 1995, BERTHOLD 1998, THOMAS \& LENNON 1999). Järvinen belegte als erster auch Änderungen in der Brutbiologie: Beim Trauerschnäpper Ficedula hypoleuca nimmt die Eigröße mit steigender Temperatur zu, was zu besseren Bruterfolgen führt (JÄRVINEN 1994, JÄRVINEN 1996). In Großbritannien wurde nachgewiesen, dass sich bei vielen Arten der Median des Legebeginns vorverlagert hat (CRICK ET AL. 1997) und dass diese Veränderungen mit der Klimaerwärmung zusammenhängen (CRICK \& SPARKS 1999). Ähnliche Trends bestehen auch in den USA (DUNN \& WINKLER 1999, BROWN ET AL. 1999). Wie erwähnt, errechnete WINKEL (1997, auch BAIRLEIN \& WINKEL 1998) eine Zunahme der Gelegegröße, der Anzahl ausgeflogener Jungvögel beim Trauerschnäpper sowie einen früheren Schlupftermin bei Kleiber Sitta europea, Kohl- und Blaumeise Parus caerulus. Vergleichbare Ergebnisse für Offenbrüter sind neben den für die Mönchsgrasmücke oben dargestellten bislang nur für Teich- und Drosselrohrsänger bekannt (METZLER 2001), bei denen sich aber ein anderes Muster ergibt, nämlich eine Erhöhung der Gelegegröße und Verkürzung der Brutsaison. Bei den belegten Veränderungen der Gelegegrößen handelte es sich stets um Zunahmen; sie sind am ehesten damit zu erklären, dass der Anteil früher und somit, unter Einfluss des Kalendereffektes der Gelegegröße, größerer Gelege in der jeweiligen Stichprobe zugenommen hat. Es sind demnach keine adaptiven Änderungen, sondern solche, die sich durch den Kalendereffekt ergeben. Gleichwohl kann eine größere Gelegegröße adaptiv sein, da mehr Junge ausfliegen und früher geschlüpfte Junge eine höhere Rekrutierungsrate aufweisen (PERRINS 1970, MøLLER 1994). In der Analyse möglicher bestandsrelevanter Konsequenzen der rezenten Klimaerwärmung steht die Forschung noch am Anfang. Einige Befunde an Meisen lassen befürchten, dass es eine Desynchronisation der Jungenaufzucht und der Hauptaufzuchtnahrung gibt, die sich negativ auf den Bruterfolg auswirkt (THOMAS ET AL. 2001, VISSER ET AL. 2002). Andere Arbeiten haben gezeigt, dass eine Vergrößerung der Eier bei warmer Witterung bessere Brut-erfolge bringen kann (JÄRVINEN 1994). Ein weiterer positiver Trend lässt sich aus den Daten von METZLER (2001) ableiten, nach denen Teich- und Drosselrohrsänger nicht nur früher brüten, sondern auch größere Gelege und einen höheren Bruterfolg haben. Veränderungen durch die Klimaerwärmung sind also deutlich (HUGHES 2000) und es wird von großem Interesse sein, weitere Vogelarten auf mögliche populationsbiologische Konsequen- 
zen hin zu untersuchen.

Vor dem Hintergrund einer möglichen Gelegegrößenselektion durch Prädation zur Risikoverteilung auf mehrere Brutversuche (SLAGSVOLD 1984) bieten die dargestellten Ergebnisse eine Interpretation der beobachteten Gelegegrößenabnahme bei der Mönchsgrasmücke seit 1981: Bei einer kurzen Brutsaison besteht eine zeitliche Einschränkung für die Sequenz von Nachgelegen, es sind weniger Nachgelege möglich als bei einer langen Brutsaison. Der Vorteil kleinerer Gelege im Hinblick auf lange Sequenzen ist also geringer. Durch Verlängerung der Brutsaison der Mönchsgrasmücke hat sich die Wahrscheinlichkeit erhöht, dass sich eine längere Sequenz von Brutversuchen lohnt. Es ergibt sich somit ein Vorteil für Individuen, die weniger Eier legen. Das Populationsmittel hätte sich im Laufe der Jahre also auch ohne Veränderung der Prädationsrate verschieben können, weil Nester mit weniger Eiern unter der vorhandenen Prädationsrate einen zunehmenden Selektionsvorteil bieten. Die verlängerte Brutsaison hat evolutive Vorteile für die Mönchsgrasmücke, weil mehr Zeit bleibt, um Gelegeverluste zu kompensieren.

Zu 2.: Beginn und Verlauf der Brutsaison hängen davon ab, wann die Weibchen ausreichend Nahrung zur Eiablage sammeln können (PERRINS 1970, NAGER 1994) und ausreichend Futter zur Jungenaufzucht vorhanden ist (HANSELL 2000, THOMAS ET AL. 2001, VISSER ET AL. 2002). Frühes Brüten bietet den Vorteil einer höheren Überlebenswahrscheinlichkeit früh geschlüpfter Jungen (PERRINS 1970, MøLLER 1994). Es ist verschiedentlich darauf hingewiesen worden, dass auch Prädation die Brutsaison beeinflussen kann, indem die Vögel auf Zeiten ausweichen, in denen weniger Prädatoren nach Nahrung suchen (SIEVING 1992, MAJOR ET AL. 1994) oder durch den in meiner Untersuchung vermuteten Effekt einer Erhöhung der Anzahl der Brutversuche (PERRINS 1970, BECHET ET AL. 1998). Aufgrund der vorliegenden Datenlage können bekannte Alterseffekte auf den Brutbeginn nicht ausgeschlossen werden. Bei vielen Vogelarten brüten ältere und erfahrenere Vögel früher als Erstbrüter (LACK 1968, CAMPBELL \& LACK 1985). Der vorliegende Trend eines früheren Beginns der Brutsaison nach einem Jahr mit hoher Prädationsrate und korrespondierend geringem Bruterfolg lässt sich somit mit einer veränderten Altersstruktur erklären: Es ist davon auszugehen, dass der Anteil einjähriger Brutvögel in einem Jahr, das auf hohe Prädationsraten folgt, geringer ist.

Ein genetischer Effekt durch eine adaptive Verschiebung des Populationsmittels ist 
unwahrscheinlich. Der Legebeginn hat nach den bekannten Untersuchungen (an Meisen) eine geringe Heritabilität (VAN NOORDWIJK ET AL. 1980, BOAG \& VAN NoORDWIJK 1987, VAN NOORDWIJK 1987), unterliegt also einer hohen phänotypischen Plastizität. Bei einem geringen Anteil an Jungvögeln ist kaum anzunehmen, dass ein Effekt statistisch von einem Jahr auf das nächste nachzuweisen ist.

Eine phänotypische Adaption kann nicht ausgeschlossen werden. Wie bereits im Kapitel 5.2.2 erwähnt, sind Singvögel in der Lage, Erfahrungen beim Bruterfolg umzusetzen, indem Neststandorte gewechselt oder beibehalten werden (MARZLUFF 1988, BOLLINGER \& GAVIN 1989). Zumindest für die Kohlmeise und den Halsbandschnäpper Ficedula albicollis ist darüber hinaus nachgewiesen, dass sie brutbiologische Parameter wie Brutbeginn, Gelegegröße und Partnertreue nach vorheriger Erfahrung optimieren (Gustafsson 1990, LINDEN 1991, WIGGINS ET AL. 1994, DHONDT \& AdRIAENSEN 1994, NAGER \& VAN NOORDWIJK 1995). Offensichtlich können außerdem die Erfahrungen anderer Brutpaare wahrgenommen und berücksichtigt werden (Stamps 1988, Julliard Et Al. 1997, Forstmeier et Al. 2001). Auch wenn die physiologischen Mechanismen für diese Phänomene ungeklärt sind, ist ein früher Beginn der Brutsaison nach einem Jahr mit hohen Prädationsraten vorstellbar. Eine Verfrühung der Brutsaison bei der Mönchsgrasmücke wäre adaptiv und es ist demnach nicht auszuschließen, dass die vorgelegte Beziehung einen Hinweis auf eine phänotypische Adaption darstellt. Im Hinblick auf die methodischen Einschränkungen muss aber offen bleiben, ob ein solcher Effekt vorliegt.

Zu 3.: Die Gelegegröße bei Nesthockern wird im Wesentlichen dadurch bestimmt, wieviele Jungvögel aufgezogen werden können, also durch Nahrungsverfügbarkeit (LACK 1948, LACK 1968). Das Konzept der „Optimal Clutch Size“ ist allgemein anerkannt (MURPhY \& HaUkiOJA 1993, Hansell 2000) und vielfach belegt worden (z.B. APARICIO 1994, MORENO \& SANZ 1994, BAKER 1995). Auch saisonale Schwankungen der Gelegegröße sind Schwankungen in der Nahrungsverfügbarkeit zuzuordnen (MURPHY 1978, CRICK ET AL. 1993, LUDVIG ET AL. 1995). Allerdings zeigen zahlreiche Arbeiten, dass die optimale Gelegegröße häufig unterschritten wird, wofür es verschiedene Gründe geben kann: Nestbauende Vögel bilden die Nestgrube durch Drehen des eigenen Körpers. Die Größe der Mulde, und damit die Aufnahmefähigkeit ist demnach durch die Größe des Körpers begrenzt (Møller 1982, Collias \& ColliaS 1984, SLAGSVOLD 1989), während bei Höhlenbrütern die Größe der Höhle maßgeblich ist (MARTIN 1993c). Auch die Habitatqualität kann individuelle Unterschiede der 
Gelegegröße bewirken (MURPHY 1978, HöGSTEDT 1980, BAKER 1994). Weitere Autoren konnten zeigen, dass die Gelegegröße einen Kompromiss zwischen der aktuellen Reproduktion und dem Lebensfortpflanzungserfolg (,lifetime-reproductivesuccess"), also zwischen dem Überleben der Jung- und Altvögel darstellt (CHARNOV \& Krebs 1974, Liou et Al. 1993, Ghalambor \& Martin 2000, Wallander \& ANDERSSON 2002). Weitere Abweichungen von der optimalen Gelegegröße entstehen durch Konkurrenz bei hohen Siedlungsdichten(BOTH ET AL. 2000), durch zusätzliche Eier, die gelegt werden, wenn die Schlüpfrate gering ist (FORBES \& MOcK 2000). Im vergangenen Jahr wurde belegt, dass auch Immunkompetenz der Alt- und Jungvögel einen Einfluss auf die Gelegegröße nehmen kann, ein Faktor, der bislang nicht beachtet wurde (MARTIN ET AL. 2001). Unterschiede gibt es auch zwischen Altersklassen: bei vielen Vogelarten legen ältere Weibchen mehr Eier (JULLIARD ET AL. 1997, CAMPBELL \& LACK 1985).

Wie in 5.1 dargestellt, wird der Prädation als eine evolutive Kraft in Lebenszyklusmerkmalen seit Mitte der achtziger Jahre größere Aufmerksamkeit geschenkt. So ist das Wachstum von Jungvögeln im Nest auf Inseln ohne Prädation langsamer als bei vergleichbaren Arten auf dem Festland (BOSQUE \& BOSQUE 1995), bei Arten, die hoher Prädation unterliegen, sind Nester kleiner und damit die Anzahl der Eier begrenzt (MøLLER 1990). Besonders kontrovers wurde diskutiert, ob Prädation ein weiterer Parameter für die Unterschreitung der optimalen Gelegegröße sein kann. MARTIN \& CLOBERT (1996) und KULESZA (1990) stellten einen Zusammenhang zwischen Gelegegröße und Prädation verschiedener geografischer Regionen fest. SoCKMAN (1997) konnte zeigen, dass kleinere Gelege einen höheren Bruterfolg hatten. Der bislang einzige Beleg für Änderungen der Gelegegröße durch Unterschiede der Prädationsrate innerhalb einer Population kommt von JULLIARD ET AL. (1997), die für die Kohlmeise eine phänotypische Anpassung der Gelegegröße an die Prädationsrate nachweisen.

Ohne Kenntnis der lokalen Gegebenheiten könnte die Beziehung zwischen der mittleren Überlebenswahrscheinlichkeit in einem Waldstück und der Gelegegröße ein Effekt der Siedlungsdichte sein: Prädationsrate und Gelegegröße können dichteabhängig sein und eine Korrelation kann sich somit zufällig und ohne biologischen Zusammenhang ergeben. Da die Unterschiede in der Prädationsrate auf lokale Unterschiede im Prädatorenspektrum zurückzuführen sind, kann dieser Erklärungsansatz zurückgewiesen werden. Nach den vorliegenden Ergebnissen wird die Gelegegröße 
bei der Mönchsgrasmücke neben saisonaler Anpassung an die Nahrungsverfügbarkeit und der Abnahme im Zuge der Klimaerwärmung auch von lokalen Unterschieden der Überlebenswahrscheinlichkeit beeinflusst und bietet damit einen weiteren Hinweis darauf, dass die Theorie von SLAGSVOLD (1984), die im interspezifischen Vergleich Gültigkeit besitzt (MARTIN \& CLOBERT 1996), auch innerhalb einer Art wirkt.

Zu 4.: Bei der Beziehung zwischen der Gelegegröße und der Überlebenswahrscheinlichkeit im Vorjahr kann ein methodischer Einfluss weitgehend ausgeschlossen werden. Eine veränderte Altersstruktur sollte nach der Regel, dass mehrjährige Altvögel größere Gelege haben zu gleich großen oder größeren Gelegen im Folgejahr führen. Dasselbe gilt für dichteabhängige Gelegegröße, wenn etwa die Brutpaardichte im Folgejahr abnimmt und damit die Nahrungskonkurrenz geringer ist. Nach Korrektur für den Kalendereffekt weist der Vergleich der natürlichen und der zusätzlichen Prädation durch Entnahme von Vögeln für Versuchszwecke auf einen genetischen oder phänotypischen adaptiven Effekt der Überlebenswahrscheinlichkeit hin, der, wie von JULLIARD ET AL. (1997) beschrieben, schon im Folgejahr zu geringeren Gelegegrößen führt.

Ein genetischer Effekt würde wirken, indem hohe Brutverluste dazu führen, dass einzene Individuen keine Nachkommen haben. Da die Nestlinge der Mönchsgrasmücke nicht laut betteln und die meisten Nester vor dem Schlupf gefunden werden, ist die Prädation nicht gelegegrößenabhängig. Der Vorteil kleinerer Gelege für die Mönchsgrasmücke muss demnach in der Einsparung von Energie liegen, die dann für Ersatzbruten zur Verfügung steht. Ausgehend davon, dass die Gelegegröße zum Teil genetisch determiniert und eine Normalverteilung in der Population zu erwarten ist, liegt der Populationsmittelwert auf einer Skala zwischen Individuen, die im Schnitt weniger bzw. mehr Eier pro Nest legen. Diese beiden „Typen“ haben in Jahren mit unterschiedlicher Prädationsrate unterschiedliche Vorteile: In Jahren mit hoher Prädation besteht für den zweiten Typ das Risiko, keine Jungvögel hochzuziehen, in Jahren mit geringerer Prädation würden mehr Jungvögel aufgezogen. Ausgehend von einer gleichen Rekrutierungsrate, hätten im ersten Fall die Nachkommen des ersten Typs einen größeren Anteil an der Population, im zweiten Fall die des zweiten. Die beobachteten Zusammenhänge zwischen Gelegegröße und der Vorjahrsprädation wären damit nicht das Resultat einer phänotypischen Anpassung sondern einer Verschiebung des Populationsmittels. 
Ein genetischer Effekt ist demnach erklärbar und kann unter Umständen für die langfristige Änderung der Gelegegröße und ihrer lokalen Unterschiede als Erklärung herangezogen werden. Einen Hinweis dafür, dass es eine genetische Komponente bei dieser Entwicklung geben könnte, liefert ein Vergleich mit Volierendaten: In einigen Jahren wurden Junge aus Nestern entnommen und im Folgejahr zur Brut in die Volieren gesetzt. Sie sind also Nachkommen der Vögel, die im Vorjahr hohen oder niedrigen Überlebenswahrscheinlichkeiten ausgesetzt waren. Die Gelegegrößen dieser Vögel zeigen wie die Freilanddaten eine Abnahme mit den Jahren, wobei die Entwicklung aus 6 Jahren zwischen 1991 und 1998 bei 123 Gelegen nicht signifikant ist, so dass sich hier weitere Untersuchungen anschließen werden.

Bei den jährlichen Änderungen ist ein solcher Effekt unwahrscheinlich. Selbst wenn die geringe Überlebenswahrscheinlickeit keinen Einfluss auf die Altersstruktur hat, weil Gelegeverluste ausgeglichen werden können, beträgt der Anteil an vorjährigen Vögeln kaum mehr als $50 \%$. Bei einer angenommenen Rückkehrrate in dieselbe Populaton von 25 \% (BAIRLEIN 1978 errechnete für die Flächen um Schloss Möggingen $8 \%$ ) würde sich bei einer Heritabilität der Gelegegröße zwischen $30 \%$ und $50 \%$ (BOAG \& VAN NOORDWIJK 1987) ähnlich wie bei der Brutsaison von einem Jahr auf das nächste kein Effekt nachweisen lassen.

Eine phänotypische Anpassung müsste durch physiologische Mechanismen wirken, die es den Altvögeln gestatten, Erfahrung früherer Brutversuche umzusetzen (JULLIARD ET AL. 1997). Da im vorliegenden Datenmaterial im Gegensatz zu JULLIARD keine individuelle Kenntlichmachung der Vögel erfolgte, kann eine endgültige Aussage, wie diese Veränderungen der Gelegegröße erfolgt sind, nicht getroffen werden.

Fazit: Die vorliegenden Zusammenhänge zwischen den brutbiologischen Parametern Gelegegröße, Brutbeginn und Überlebenswahrscheinlichkeit deuten auf ein Wirkungsgefüge bei der Mönchsgrasmücke hin, dass kurzfristige phänotypische und langfristige genetische Adaptionen an Schwankungen der Überlebenswahrscheinlichkeit ermöglicht. Das vorliegende Datenmaterial gestattet wegen fehlender individueller Kenntlichmachung keine abschließende Bewertung; es sind weitere Analysen des Datenmaterials aus Volieren und genauere Freilanderhebungen erforderlich, um die verbleibenden Zweifel, ob die errechneten Korrelationen biologische Relevanz besitzen und es damit eine Risikoverteilung bei der Mönchsgrasmücke gibt, auszuräumen. 


\subsection{Ist die Mönchsgrasmücke durch den Eichelhäher bedroht?}

Es besteht seit langem eine heftige und sehr emotional geführte Diskussion darüber, ob Rabenvögel wie Aaskrähe, Elster und Eichelhäher für das Verschwinden vieler Vogelarten aus Teilen unserer Landschaft verantwortlich sind (DEUTSCHER JAGDSCHUTZVERBAND E.V. 1997, MÄCK \& JÜRGENS 1999). Es gibt zahlreiche Untersuchungen, die darauf hinweisen, dass ihr Einfluss geringer ist als angenommen (z.B. KoOIKer 1994, Bellebaum \& EIKHORSt 2000, Bellebaum 2001, BlühdORN 2001. SCHÖNENBERGER, mündl.). Für Wiesenbrüter scheinen vor allem nachtaktive Tiere als Prädatoren in Frage zu kommen, was Rabenvögel ausschließt (BellebAum \& EIKHORST 2000, SCHÖNENBERGER mündl.). BAUER \& LEY (1994) legen dar, dass es generell keine Hinweise auf Bestandsgefährdungen durch Prädation gibt. Einige Arbeiten belegen hohe Prädationsraten durch Rabenvögel (HUBER 1956) und einen Einfluss auf den Bestand einer anderen Vogelart (HOI 1999). Auch wenn die Nahrungszusammensetzung nicht darauf hindeutet, dass Eier und Nestlinge die Hauptnahrung für Eichelhäher sind (GLUTZ VON BLOTZHEIM \& BAUER 1993), ist umgekehrt bislang kaum untersucht worden, in welchem Ausmaß sie tatsächlich Nester ausnehmen.

Die Ergebnisse der vorliegenden Arbeit belegen einen hohen quantifizierbaren Einfluss des Eichelhähers auf Nestverluste. Es ist daher angebracht, auf diese Thematik einzugehen und aufzuzeigen, inwieweit der Eichelhäher für die Mönchsgrasmücke eine Bedrohung darstellt.

1. Die saisonale Abnahme der Prädationsrate deutet darauf hin, dass Nestersuche mit abnehmender Nesterdichte weniger effizient wird, weil Handhabungszeit und Suchzeit mehr Energie verbrauchen, als durch die gefundene Nahrung gewonnen werden kann. Spätestens dann weichen die Prädatoren auf andere Nahrungsquellen aus. Nester, die danach ausgeräumt werden, wären dann eher zufällige Funde.

2. Auch unabhängig vom eben beschriebenen Phänomen ist die Mönchsgrasmücke in der Lage, Brutverluste während der Saison auszugleichen. Vier Ersatzbruten sind dabei nicht die Ausnahme und wir können davon ausgehen, dass Bruten, die noch im Juli angelegt werden, erfolgreich sind. Die Zusammenhänge zwischen Prädationsrate und Brutsaison bzw. Gelegegröße und Ausdehnung der Brutsaison unter Reduktion der Gelegegröße weisen zudem darauf hin, dass es kom- 
pensatorische Effekte gibt, die, bezogen auf die Populationsdynamik, vermuten lassen, dass Mechanismen zur Kompensation von Gelegeverlusten ggf. höhere Verluste in einem Jahr im Folgejahr ausgleichen können.

3. Der Vergleich der Untersuchungsflächen zeigt zwar, dass das Fehlen von Eichelhähern zu einer verminderten Prädation führt, gleichzeitig gehen in diesen Waldstücken Nester häufiger verloren als durch das Fehlen des Eichelhähers zu erwarten wäre. Das hängt gegebenenfalls mit einer kompensatorischen Funktion der verbleibenden Prädatoren Steinmarder, Fuchs etc. zusammen (PARKER 1984), die, wie dargelegt, Nester im Schnitt später finden als der Eichelhäher. Das bedeutet einerseits, dass der Eichelhäher einige Nester findet, die sonst später von anderen gefressen worden wären, dass aber andererseits die Nestverluste in einem späteren Stadium wesentlich „teurer" sind, da schon mehr in die Brut investiert wurde. Es ist somit günstiger, wenn ein Nest in einem früheren Stadium gefunden wird. Auf diese Weise wird der Effekt eines geringeren Prädationsdruckes durch den Eichelhäher zum Teil nivelliert. Eine Regulation durch Abschuss hätte nach diesen Ausführungen einen geringen Effekt. Auch die Ergebnisse von Mallorca oder Madeira liefern hier keine Gegenargumente. Es ist unbekannt, wie lange es dauern würde, Neststandorte so zu verändern, dass beim völligen Fehlen von Rabenvögeln auch Säugetiere ihren Einfluss verlören. Es ist unwahrscheinlich, dass innerhalb einer Saison oder weniger Jahre Effekte für die Neststandortwahl erzielt werden können. Reviere, in denen die Eichelhäher entfernt wären, würden darüber hinaus vermutlich von nicht verpaarten Eichelhähern besetzt oder unter Nachbarn aufgeteilt, was zwar die Dichte vermindert, aber nicht zu einem völligen Fehlen führt.

Die Mönchsgrasmücke ist eine häufige Art mit steigenden Beständen (BAUER ET AL. 2002 im Druck). Es schließt sich die Frage an, ob die Prädation durch Rabenvögel auf andere, seltenere und gefährdete Vogelarten einen größeren Einfluss haben könnte. Ausgehend von der allgemein geringeren Siedlungsdichte seltener Arten ist zu vermuten, dass insgesamt weniger Nester durch Prädation verloren gehen. Darüber hinaus findet der Eichelhäher, wie in der vorliegenden Arbeit nachgewiesen, eher hohe als niedrige Nester. Bodennester, das darf aus den vorliegenden Ergebnissen gefolgert werden, unterliegen einer geringen Prädation durch den Eichelhäher. Schon Dorngrasmücke und Goldammer haben erheblich niedrigere Brutverluste als Amseln oder Mönchsgrasmücken in den gleichen Flächen (BARKOW ET AL. 2001). 
Es ist somit unwahrscheinlich, dass durch die Regulation von Eichelhähern ein Vorteil für die Mönchsgrasmücke oder andere Arten erzielt würde. Effektiv wäre es dagegen, das Ausmaß vermeidbarer Brutverluste durch anthropogene Störungen zu vermindern. Wie dargelegt, sind $20 \%$ der Brutverluste nicht durch Prädation hervorgerufen, sondern durch andere Faktoren: Davon sind mindestens $10 \%$ direkten Einflussnahmen des Menschen durch Waldrandpflege, das sogenannte Abschlegeln, zuzurechnen, das in einigen Untersuchungsgebieten während der Brutsaison durchgeführt wurde. Dazu kommen Verluste durch Mahd, wodurch Bruten zerstört oder freigelegt werden, so dass sie Witterungseinflüssen schutzlos ausgeliefert sind. BARKOW (2001a) legt dar, dass diese Störungen mit sinkender Nesthöhe zunehmen und niedrig brütende Arten weit mehr davon betroffen sind. Auch wenn PÄTZOLD (1983) bei der Feldlerche $15 \%$ Verluste durch Einwirkung der Bewirtschaftung gering schätzt, können solche zusätzlichen Verluste dann unter Umständen nicht mehr kompensiert werden, zumal wenn die Einflussnahme große Teile des Revieres betrifft und es sich evtl. um Weitstreckenzieher mit einer kurzen Brutsaison handelt (SCHULZE-HAGEN ET AL. 1996). Des weiteren können durch Störungen im Brutrevier oder in Nestnähe Mäuse Zugriff auf eine Brut erhalten, die im Normalfall hätte verteidigt werden können (SCHAEFER im Druck). 



\section{Zusammenfassung}

Nestprädation gilt als ein Hauptparameter für die Selektion von Lebenszyklusmerkmalen bei Singvögeln. Als Reaktion kann die natürliche Selektion zum einen eine Vermeidung der Prädation bewirken, z.B. durch das heimliche Verhalten der Eltern, Verteidigung der Nester, Verminderung der Auffälligkeit eines Nestes durch Verringerung der Gelegegröße und durch die Wahl geeigneter Neststandorte. Weiterhin können evolutive Vorteile erzielt werden, indem der Effekt der Prädation durch schnelle Kompensation von Verlusten sowie eine Risikoverteilung („bet hedging“) auf mehrere Bruten - unter Verringerung der Gelegegröße und Verlängerung der Brutsaison vermindert wird. Dabei sind die jeweiligen Prädatoren entscheidend dafür, welche Strategie für einen Singvogel adaptiv ist.

Neben der Auswertung von 2315 Nestfunden der Mönchsgrasmücke Sylvia atricapilla, die an der Max-Planck-Forschungsstelle für Ornithologie, Vogelwarte Radolfzell, seit 1981 aus sieben verschiedenen Untersuchungsflächen im westlichen Bodenseeraum, Südwestdeutschland, aufgezeichnet wurden, wurden zwischen 1999 und 2001 in demselben Gebiet im Rahmen einer Freilandstudie zur Bestimmung der Prädatoren 201 Nester gesucht, Neststandortparameter aufgenommen, 126 aktive Nester mit Videosystemen überwacht und 66-mal Fotofallen an 36 beköderten $\mathrm{Ne}$ stern installiert. Zur Aufdeckung möglicher Multiprädatoreneffekte auf Neststandortselektion wurde außerdem im Frühjahr 2001 eine Vergleichsuntersuchung an $64 \mathrm{Ne}$ stern auf Mallorca durchgeführt. Folgende Ergebnisse können zusammenfassend formuliert werden:

1. Prädationsraten: Die Verlustrate beträgt ca. $50 \%$, wobei nach den Videoaufzeichnungen etwa 80 \% der Verluste Prädationen zuzuordnen sind. Die Überlebenswahrscheinlickeit eines Nestes beläuft sich auf 0,23 (Nestfunde, $n=1492$ ) und 0,24 (Freilandstudie, $n=193$ ). Die meisten Verluste sind in der Brutphase zu verzeichnen: mit einer Wahrscheinlichkeit von 0,36 (Nestfunde) bzw. 0,41 (Freilandstudie) der angelegten Nester schlüpfen Junge, die dann mit einer Wahrscheinlichkeit von 0,63 bzw 0,59 ausfliegen. Die Überlebenswahrscheinlichkeit steigt mit der Saison und scheint dichteabhängig zu sein, d.h. je weniger Nester vorhanden sind, desto höher ist die Überlebenswahrscheinlichkeit. Über einen Zeitraum von 20 Jahren schwankt die Überlebenswahrscheinlichkeit, zeigt aber keinen Trend. Auf Mallorca wurde keine Prädation festgestellt, was z.T. methodi- 
schen Einschränkungen zuzuordnen ist. Vergleiche mit Madeira, wo ähnliche Verhältnisse vorliegen, zeigen, dass die Überlebenswahrscheinlichkeit mehr als doppelt so hoch ist.

2. Prädatoren: Aus den Videoaufzeichnungen geht hervor, dass Eichelhäher Garrulus glandarius für $46 \%$ der Brutverluste und damit für $22 \%$ der Resultate von Brutversuchen der Mönchsgrasmücke verantwortlich sind. Weitere Prädatoren sind Steinmarder, Fuchs, Aaskrähe, Waldmaus, Mauswiesel, Wildschwein und Waldkauz (aus einer Vorstudie sind zudem Haselmaus, Dachs, Hermelin und Neuntöter - nach der Häufigkeit ihres Auftretens - zu nennen). Dass Eichelhäher häufig mehrmals zum Nest kommen, um Eier oder Jungvögel zu holen, lässt den Schluss zu, dass sie Nester systematisch suchen und wiederfinden.

3. Lokale Unterschiede der Überlebenswahrscheinlichkeit: Die Überlebenswahrscheinlichkeit zwischen den einzelnen Untersuchungsflächen der Nestfunde variiert. Es gibt einen signifikanten Zusammenhang zwischen der Größe der Flächen und Hinweise dafür, dass Landschaftsstruktur einen Einfluss ausübt: Je kleiner und isolierter eine Fläche, desto höher die Überlebenswahrscheinlichkeit. Eine Erklärung für diesen Zusammenhang bieten laut Literatur die Habitatansprüche des Eichelhähers, dessen Reviere nicht unter 10 ha groß sind und der kleine, isoliert liegende Flächen wegen der Gefahr durch Habichte und Sperber selten aufsucht.

4. Anpassungen des Verhaltens: Die Altvögel verhalten sich beim Herannahen eines Feindes ruhig, ducken sich ins Nest und fliegen erst auf, wenn der Prädator das Nest bereits erreicht hat. Der Vergleich mit den Ergebnissen der Fotofallen, bei denen die Hälfte der dokumentierten Ereignisse durch Wald- und Haselmäuse verursacht war, zeigt, dass Mönchsgrasmücken offensichtlich in der Lage sind, Mäuse vom Nest fernzuhalten. Mäuse wurden auf allen Flächen nachgewiesen und erschienen auch regelmäßig in Nestnähe. An aktiven Nestern gab es nur in zwei Fällen einen Verlust durch Mäuse. Die Überlebenswahrscheinlichkeit großer Gelege unterscheidet sich nicht von der kleiner Gelege. Es kann ausgeschlossen werden, dass ein Nest durch ein kleineres Gelege und korrespondierend verminderte Fütterraten unauffälliger wird, weil die Überlebenswahrscheinlichkeit vor dem Schlupf wesentlich geringer ist als danach.

5. Neststandortselektion: Die gemessenen Neststandortparameter (Nest- und Nist- 
pflanzenhöhe, Breite der Nistpflanze und Entfernung eines Nestes zum Rand der Nistpflanze, Minima, Maxima und Mittelwerte der Sichtbarkeit aus verschiedenen Richtungen, Entfernung zum Waldrand und alternative Neststandorte) können mit dem Bruterfolg ebensowenig in Verbindung gebracht werden, wie extreme und mittlere Standorte Unterschiede im Bruterfolg zeigen. Lediglich die Wahl der Nistpflanze zeigt, dass die Mönchsgrasmücke eine Selektion von Neststandorten durchführt: häufig genutzte Neststandorte haben einen deutlich höheren Bruterfolg als selten genutzte. Die Neststandortselektion der Mönchsgrasmücke ist das Resultat eines Multi-Prädatoren-Effektes: Durch Videoaufnahmen kann belegt werden, dass Säuger eher niedrig und zentral, Rabenvögel eher hoch und randständig angelegte Nester ausnehmen. Die Werte für Nesthöhe und Randständigkeit erfolgreicher Nester ordnen sich zwischen denen dieser Prädatorengruppen ein. Auf Mallorca werden die Nester dort gebaut, wo sie nach den Ergebnissen aus Südwestdeutschland am sichersten vor bodennah suchenden Säugern sind: weit oben und im äußeren Bereich einer Nistpflanze an Standorten, die am ehesten vom Eichelhäher gefunden würden. Sie sind vergleichsweise gut sichtbar, was darauf hinweist, dass ein Verstecken der Nester nicht erforderlich ist, weil keine nestraubenden Rabenvögel auf der Insel vorkommen.

6. Kompensation von Brutverlusten: Wie Befunde aus den Volieren der Vogelwarte Radolfzell zeigen, können die Gelegeverluste innerhalb einer Saison kompensiert werden: Der Median der Nachlegedauer beträgt hier 6 Tage. Da ein Nest 10,5 Tage nach dem Legebeginn ausgefressen wird, dauert ein Brutversuch im Schnitt 16,5 Tage. Die mittlere Anzahl möglicher Brutversuche pro Saison liegt daher bei etwa 4,4 .

7. Risikoverteilung: Die Ergebnisse der Brutbiologie und Neststandortcharakteristika am Bodensee und auf Mallorca bestätigen im Wesentlichen bekannte Befunde. Allerdings haben sich seit 1981 und im Vergleich mit der Literatur in Südwestdeutschland Veränderungen infolge der Klimaerwärmung ergeben, die eine Ausdehnung der Brutsaison und eine Verminderung der Gelegegröße belegen. Es können vier Zusammenhänge aufgezeigt werden, die dafür sprechen, dass eine Risikoverteilung bei der Mönchsgrasmücke vorliegt: 1. Die Brutsaison dehnt sich bei gleichzeitiger Reduktion der Gelegegröße aus. 2. Nach einem Jahr mit geringer Überlebenswahrscheinlichkeit beginnt die Brutsaison früher. 3. Je geringer die über Jahre gemittelte Überlebenswahrscheinlichkeit auf einer Untersuchungs- 
fläche ist, desto geringer ist die mittlere Gelegegröße. 4. Nach einem Jahr mit geringer Überlebenswahrscheinlichkeit ist die Gelegegröße geringer. Unter Berücksichtigung methodischer Einschränkungen und bei biologischer Relevanz der errechneten Korrelationen deuten diese Befunde auf ein Wirkungsgefüge hin, das die Gelegegröße der Mönchsgrasmücke unter dem Einfluss der Überlebenswahrscheinlichkeit optimiert, um mit einer höheren Sequenz von Brutversuchen Brutverluste ausgleichen zu können.

Fazit: Prädationsrate und -muster unterliegen aufgrund unterschiedlicher Prädatorenspektren lokalen und regionalen Schwankungen. Adaptionen, die die Prädationsrate vermindern können, sind bei der Mönchsgrasmücke unauffälliges Verhalten, Verteidigung und Neststandortselektion. Der Effekt der Prädation wird durch Selektion schneller Kompensationsfähigeit der Brutverluste und vermutlich einer Risikoverteilung auf mehrere Brutversuche vermindert.

Die Frage danach, ob die Ergebnisse für eine Populationskontrolle des Eichelhähers sprechen, muss ausdrücklich verneint werden, weil die Mönchsgrasmücke in der Lage ist, Gelegeverluste zu kompensieren. Für andere Arten, besonders für gefährdete Bodenbrüter, ist ein negativer Effekt durch den Eichelhäher unwahrscheinlich, da er vor allem hohe und außen liegende Nester prädiert. 


\section{Summary}

Nest predation is one of the main selective forces in life history evolution of songbirds. On the one hand natural selection can favour traits which lower predation, e.g. by cryptic behaviour of adult birds, defence of nests, reducing the number of eggs and nestlings to make the nest less conspicuous and by the choice of suitable nest sites. Beside this, evolutionary advantages can be achieved by reducing the effect of predation through the ability to quickly compensate for nest losses, and through bet hedging to several broods. In any case, the predators in a given area determine, which of the strategies will be adaptive.

In this study, firstly, I analysed 2300 nest records of the Blackcap (Sylvia atricapilla) from the Max Planck Research Unit for Ornithology, Vogelwarte Radolfzell. The nests were found between 1981 and 2000 in seven woodlots at the Western Lake of Constance, south-western Germany. Secondly, in the same area between 1999 and 2001201 blackcap nests were observed in order to uncover causes of nesting failures and nest predators. I took nest site parameters, monitored 130 active nests with videocameras and baited 37 abandoned, real nests to be monitored with phototraps. To analyse possible multi-predator effects on nest site selection I made a comparative study on Mallorca, Spain, in spring 2001.

The results can be summarised as followed:

1. Predation rates: $50 \%$ of blackcaps nests get lost before the young have fledged. About $80 \%$ of nest losses are due to predation. The survival probability of a nest amounts to 0.23 ( $n=1492$, nest records) or 0.24 ( $n=193$, field study). Most nests get lost during incubation, so, with a probability of 0.36 (nest records) or 0.41 (field study) young will hatch from eggs that fledge with a probability of 0.63 or 0.59 , respectively. The survival probability increases as the season proceeds and seems to decrease with density of nests in the study site. There is no trend between 1981 and 2000, but survival probability differs from year to year. I did not find any predation on Mallorca, which can be explained by methodological constraints. Survival probability is more than twice as high on Madeira, where the situation is comparable.

2. Predators: The recordings from video-monitored nests show that the Eurasian jay 
(Garrulus glandarius) causes $46 \%$ of the nest losses and is thus responsible for the outcome of $22 \%$ of nesting attempts. Additionally, nest predation was found to involve stone marten, red fox, common crow, mice, least weasel, wild boar and tawny owl (in a pilot study in the same area dormouse, badger, stoat and red backed shrike were also recorded). As jays hardly take eggs and young at once but return several times until all nest contents are removed, I assume that they search for nests systematically and, once discovered, are able to remember nest sites.

3. Differences in survival probability between study sites: The survival probability of nests differs between the seven study sites. There is a significant correlation between patch size and predation and furthermore there seems to be an impact of landscape structure: the smaller and more isolated a patch, the higher the survival probability within the patch. An explanation for this pattern can be derived from literature: territories of the main predator, the jay are usually larger than 10 ha. Small and isolated patches are avoided by the jay due to the danger of being predated by sparrowhawks and goshawks.

4. Adult behaviour: parental birds stay quiet on and duck themselves into the nest when a predator approaches to fly off in the last moment. Comparison of the video-recordings with results from phototraps show, that blackcaps apparently are able to prevent small mammals to access the nest: more than $50 \%$ of the events documented by photographs were due to mice, whereas in video-recordings mice amounted to only 2 out of 45 cases. Clutches of different size do not differ in their survival probability, so we can exclude that a smaller clutch may be less conspicuous because corresponding feeding rates are lower. Survival probability is much higher during incubation than during chick feeding stages.

5. Nest site selection: none of the nest site characteristics measured (nest height, height of nesting plant, diameter of nesting plant, distance of nest to the edge of the nesting plant, minimum, mean and maximum visibility from six directions, distance to forest edge and edge of nesting structure) corresponded with nesting success. Neither did extreme or average values of the characteristics reveal differences in nesting success. Only the choice of nesting plant seems to have an impact on nesting success, as plants used regularly have significantly fewer losses than those used rarely. Nest site selection in the blackcap results from a 
multi-predator effect: nests predated by mammals are less elevated and have a more central position in the nesting plant, whereas corvids predate in more elevated and peripheral nests. Successful nests average between those values. On Mallorca corvids are missing. As a consequence nests are usually easily visible, more elevated and peripheral than in Southern Germany. So, on Mallorca the blackcap chooses nest sites which were most likely to be discovered by jays but safe from mammals.

6. Compensation of nest losses: Results from the aviaries of the Vogelwarte Radolfzell show that losses can easily be offset within a breeding season: the median time between nest loss to relaying was 6 days. As in the wild a nest is predated on average 10.5 days after the onset of laying, so an average breeding attempt lasts 16.5 days. The number of breeding attempts per season may amounts to 4.4 .

7. Bet hedging: the data on breeding biology and nest site selection match with data published elsewhere. But regarding the breeding season and clutch size the nest records reveal changes as compered to the literature. Those changes are most likely due to the recent climate warming and result in a extension of breeding season, with the season starting earlier and lasting longer, and a decrease in clutch size. Four correlations give evidence for bet hedging in the blackcap: 1 . Breeding season extends while clutch size decreases; 2. Breeding season starts earlier following a year with low survival probability; 3. Average survival probability correlates with corresponding clutch size in the study sites; and 4. Clutch size is lower following a year with low survival probability. Taking into account methodological constraints and biological relevance of these results, we have evidence for a mechanism that optimises clutch size under the impact of predation. This mechanism is supposed to allow more breeding attempts per season to compensate for nesting failures.

Conclusion: Due to differences in predator ranges, patterns and rates of predation differ on a local and regional scale. The blackcap may reduce nest predation by inconspicuous behaviour, nest defence against small mammals and adaptive nest site selection. The effect of predation may be lowered by laying quick replacement clutches and, as to be supposed, by bet hedging to several breeding attempts. 
From a conservation point of view, there is no evidence supporting population control of corvids were necessary since blackcaps can compensate for the nest losses. The probability is low that corvids are harmful for other, mainly ground-nesting species since they mainly predate on elevated and peripheral nests. 


\section{Danksagung}

Viele Menschen haben durch Einwürfe, Ideen, Vorschläge und manchmal auch nur durch eine gerunzelte Stirn zum Entstehen dieser Arbeit beigetragen. Allen diesen möchte ich herzlich danken!

Besonders bedanken möchte ich mich bei Prof. Dr. P. Berthold und zwar für die exklusiven Forschungsbedingungen, die mir während dieser Arbeit geboten wurden, für die von mir als ausgezeichnet empfundene Betreuung, inklusive der konstanten Versorgung mit aktueller Literatur, und schließlich möchte ich Frau Mohr und Herrn Berthold für das große Interesse danken, das sie meiner Arbeit entgegen gebracht haben.

Herrn Prof. Dr. M. Schaefer danke ich für die anstandslose Übernahme des Referates und die freundliche und selbstverständliche Abwicklung aller fachlichen und bürokratischen Fragen, die sich im Laufe der drei Jahre ergeben haben.

Herrn Prof. Dr. E. Schürmann danke ich für die spontane Übernahme des Korreferats.

Der Familie von Bodmann möchte ich ganz herzlich dafür Dank sagen, dass sie mich in ihrem „Garten" haben filmen lassen und dass sie gewisse Einschränkungen z.B. bei der Jagd in Kauf genommen haben, wenn ich zu allen möglichen Tag- und Nachtzeiten aufgetaucht bin, um Akkus zu wechseln oder Nester zu suchen.

Ein weiterer großer Dank gebührt allen Mitarbeitern der Vogelwarte Radolfzell! Zunächst dafür, dass sie mich im Mai 1999 so herzlich aufgenommen haben, was für entspanntes Arbeiten unabdingbar ist. Viel wichtiger aber sind die Kleinigkeiten, die sich ständig ergeben und die "immer auch noch“ zu machen sind und um die ich mich an einer anderen Stelle selber hätte kümmern müssen. Das reichte von „A“ wie „Vollgetanktes, fahrbereites Auto vorhanden“ bis „Z“ wie „sauberes Zimmer“.

Besonders dankbar aber bin ich Herrn Ulrich Querner, der jederzeit für Probleme, Fragen und Einschätzungen zur Verfügung stand und der, was die praktische Durchführung dieser Arbeit angeht, einen großen Beitrag geleistet hat!

Weiterhin möchte ich Herrn Dr. Wolfgang Fiedler meinen Dank aussprechen, der ähnlich wie Herr Querner für viele Probleme eine Lösung parat hatte und sich immer die Zeit genommen hat, Probleme anzuhören und Lösungsvorschläge zu unterbrei- 
ten.

Dr. Hans-Günther Bauer, Dr. Tim Coppack, Dr. Wolfgang Forstmeier, PD Dr. Bernd Leiser, Dr. Willy Ley und Dr. Francisco Pulido bin ich für viele klärende inhaltliche Gespräche und Willy darüber hinaus für die Durchsicht des Manuskriptes dankbar. Im Bezug auf statistische Fragen danke ich besonders Francisco für die Hot-Line!

Georg Heine von der Universität Konstanz danke ich ganz herzlich für die technische Unterstützung. Wenn es so richtig an Eingemachte ging, war immer Zeit und eine Lösung parat.

Dr. Willi Nagl, ebenfalls Uni-Konstanz, danke ich für die Zeit, die er sich genommen hat, um mich auf den Sprung in das Programm „Jump“ vorzubereiten und für die weitergehende Einführung in multivariate Statistik.

Dr. Andy Barkow möchte ich ganz besonders danken. Nicht nur, weil er zwischen Weihnachten und Neujahr 1998 die Ausschreibung für diese Arbeit entdeckte, sondern vor allem für die tägliche wissenschaftlichen Auseinandersetzung mit dem Thema.

Am Ende danke ich meiner Familie: Besonders meinen Eltern bin ich für ideelle und finanzielle Unterstützung und Begleitung während des gesamten Studiums bis heute sehr, sehr dankbar!

Alfred Schulz danke ich für die hingebungsvolle Korrektur des Manuskriptes.

Meiner lieben Sinje danke ich dafür, dass sie ganz selbstverständlich drei Jahre eine Rundumbetreuung geleistet und eigene Wünsche in den Hintergrund gestellt hat. Ohne dies und die ständige Rückendeckung durch sie wäre manches nicht möglich gewesen. Stina danke ich für die wackere Begleitung im Feld („Ich glaube, das ist hier nicht für Kinder.“) und Malin für viele Tage der Entbehrung („Papa nein tschüss!“).

Die Untersuchungen in Deutschland wurden durch ein Doktorandenstipendium der Max-Planck-Gesellschaft gefördert, die Untersuchungen auf Mallorca durch ein Stipendium des DAAD zur wissenschaftlichen Aus- und Fortbildung im Ausland. 


\section{Literaturverzeichnis}

Anderson TR 1979: Experimental synchronisation of sparrow reproduction. Wilson Bulletin 91: 317 - 319.

Andrén H 1990: Despotic distribution, unequal reproductive success, and population regulation in the jay Garrulus glandarius L.. Ecology 71: 1796 - 1803.

Andrén H 1992: Corvid density and nest predation in relation to forest fragmentation: a landscape perspective. Ecology 73: 794 - 804.

Andrén H 1994: Effects of habitat fragmentation on birds and mammals in landscapes with different proportion of suitable habitat: a review. Oikos 71: 355 366.

Andrén H 1995: Effects of landscape composition on predation rates at habitat edges. Chapman \& Hall, London. S. 225 - 255.

Andrén H, P Angelstam, E Lindström, und P Widén 1985: Differences in predation pressure in relation to habitat fragmentation: an experiment. Oikos 45: 273 277.

Angelstam P 1986: Predation on ground nesting birds'nests in relation to predator densities and habitat adge. Oikos 47: 365 - 373.

Aparicio JM 1994: The seasonal decline in clutch size: an experiment with supplementary food in the kestrel, Falco tinnunculus. Oikos 71: 451 - 458.

Askins RA, JF Lynch und R Greenberg 1990: Population declines in migratory birds in eastern North America. Current Ornithology 7: 1 - 57.

Bairlein F 1978: Über die Biologie einer südwestdeutsche Population der Mönchsgrasmücke (Sylvia atricapilla). Journal für Ornithologie 119: 14 - 51.

Bairlein F, P Berthold, U Querner und R Schlenker 1980: Die Brutbiologie der Grasmücken Sylvia atricapilla, borin, communis und curruca in Mittel - und NEuropa. Journal für Ornithologie 121: 325 - 369.

Bairlein F und W Winkel 1998: Vögel und Klimaveränderungen. In: Lozan, J.L, GraßI, H.\& Hupfer, P.(eds.): Warnsignal Klima S. 281 - 285.

Baker BW 1980: Hair-chatchers aid identifying mammalian predators of groundnesting birds. Wildlife Society Bulletin 8: 257 - 259.

Baker M 1994: Phenotypic plasticity in laying date and clutch size in house sparrows. Journal für Ornithologie 135: 9.

Baker M 1995: Environmental component of latitudinal clutch-size variation in House Sparrows (Passer domesticus). Auk 112: 249 - 252.

Ball IJ, RJ Gazda und DB Mcintosh 1994: A simple device for measuring survival time of artificial. Journal of Wildlife Management 58: 793 - 796.

Barkow A 2001a: First class aus zweiter Hand? Vortrag auf dem 6.Symposium Ethologie und Naturschutz der Ethologischen Gesellschaft e.V.

Barkow A 2001b: Die ökologische Bedeutung von Hecken für Vögel. Dissertation zur Erlangung des naturwissenchaftlichen Doktorgrades an der Georg August Universität Göttingen. 
Barkow A, F Bairlein und M Mühlenberg 2001: Prädationsraten an Vogelnestern in Hecken. E.Gottschalk, A.Barkow, M.Mühlenberg \& J.Settele (Hrsg.2001): Naturschutz und Verhalten.UFZ-Bericht 2/2000. 111 - 115.

Bauchau V und I Seinen 1997: Clutch desertion and re-nesting in pied flycatchers: An experiment with progressive clutch removal. Animal Behaviour 54: 153 161.

Bauer H-G und P Berthold 1997: Die Brutvögel Mitteleuropas. Bestand und Gefährdung. Aula Verlag, Wiesbaden

Bauer H-G, P Berthold, P Boye, W Knief, P Südbeck und K Witt 2002: Rote Liste der Brutvögel Deutschlands - 3. überarbeitete Fassung, 31.12.2001. Berichte zum Vogelschutz 39: im Druck.

Bauer H-G und H-W Ley 1994: Haben zwischenartliche Konkurrenz und Prädation eine Bedeutung für den Rückgang der Vogelarten? Zeitschrift für Ökologie und Naturschutz 3: 61 - 69.

Bayne EM und KA Hobson 1997a: Comparing the effects of landscape fragmentation by forestry and agriculture on predation of artificial nests. Conservation Biology $11: 1418$ - 1429 .

Bayne EM und KA Hobson 1997b: Temporal patterns of predation on artificial nests in the southern boreal forest. Journal of Wildlife Management 61:1227 - 1234.

Bayne EM, KA Hobson und P Fargey 1997: Predation on artificial nests in relation. Ecography 20: 233 - 239.

Bechet A, P Isenmann, und R Gaudin 1998: Nest predation, temporal and spatial breeding strategy in the woodchat shrike Lanuis senator in mediterranean France. Acta Oecologica 19: 81 - 87.

Begon M, JL Harper und CR Townsend 1991: Ökologie. Birkhäuser Verlag, Basel.

Bellebaum J 2001: Wer stiehlt Eier wirklich? Falke 48: 138 - 141.

Bellebaum J und W Eikhorst 2000: Krähen in Wiesenvogelschutzgebieten - ein Problem in Ost und West? 133. Internationale Jahresversammlung der Deutschen Ornithologen-Gesellschaft in Leipzig

Bennett ATD 1993: Spatial memory in a food storing corvid: I. Near tall landmarks are primarily used. Journal of Comparative Physiology A - Sensory Neural \& Behavioral Physiology. 173: $207-214$.

Berg A 1996: Predation on artificial, solitary and aggregated wader nests on farmland. Oecologia 107: 343 - 346.

Berthold P 1976: Methoden der Bestandserfassung in der Ornithologie: Übersicht und kritische Betrachtung. Journal für Ornithologie 117: 1 - 69.

Berthold P 1977a: Der Bruterfolg von Freibrütern bei regelmäßiger Nesterkontrolle. Journal für Ornithologie 118: 204 - 204.

Berthold P 1977b: Steuerung der Jugendentwicklung bei verschiedenen Populationen derselben Art: Untersuchungen an südfinnischen und südwestdeutschen Gartengrasmücken Sylvia borin. Die Vogelwarte 29: 38 - 44.

Berthold P 1978: Brutbiologische Studien an Grasmücken: Über die Nistplatzwahl der Mönchsgrasmücke Sylvia atricapilla im Fichten - Picea abies - Wald. Journal für Ornithologie 119: 287 - 297. 
Berthold P 1990: Patterns of avian migration in light of current global "green house" effects: A central European Perspective. Acta XX Congr.Int.Orn. 780 - 786.

Berthold P 1998: Vogelwelt und Klima: gegenwärtige Veränderungen. Naturwissenschaftliche Rundschau 51: 337 - 346.

Berthold P 1999: A comprehensive theory of the evolution, control and adaptability of avian migration. In: Adams N. und R Slotow (Hrsg.), Proceedings of the 22 International Ornithological Congress Durban, University of Natal: Ostrich 70: $1-11$

Berthold P 2000: Vogelzug. Wissenschaftliche Buchgesellschaft, Darmstadt.

Berthold P und U Querner 1978: Über die Brutleistung der Mönchsgrasmücke Sylvia atricapilla. Journal für Ornithologie 119: 114 - 114.

Berthold P und U Querner 1984: Minimale Nestabstände bei Garten- und Mönchsgrasmücke (Sylvia borin und S. atricapilla). Die Vogelwarte 32: 304 - 305.

Berthold P, U Querner und R Schlenker 1990: Die Mönchsgrasmücke. A. Ziemsen Verlag, Wittenberg Lutherstadt 1:

Best LB und DF Stauffer 1980: Factors affecting nesting success in riparian bird communities. Condor 82: 149 - 158.

Bezzel E 1993: Kompendium der Vögel Mitteleuropas. Aula Verlag, Wiesbaden

Bezzel E und W Jenz 1995: Verschiebung der Wegzugperiode bei der Mönchsgrasmücke (Sylvia atricapilla) 1966 - 1993 - Reaktion auf die Klimaerwärmung? Journal für Ornithologie 136: 83 - 87.

Bibby CJ, ND Burgess und DA Hill 1993: Bird Census Techniques. Academic Press Ltd, London.

Blühdorn I 2001: ohne Titel. Jahresberichte der Biologischen Station Rieselfelder Münster.

Boag PT und AJ van Noordwijk 1987: 2 Quantitative genetics. In: Cooke F und PA Buckley (Hrsg.): Avian genetics. A population and ecological approach. Academic Press, London. S. 57 - 65.

Bollinger E und TA Gavin 1989: The effects of site quality on breeding-site fidelity in bobolinks. Auk 103: 584 - 594.

Bortz J 1993: Statistik. Springer Verlag, Berlin, Heidelberg, New York.

Bosque C und MT Bosque 1995: Nest predation as an selective factor in the evolution of developmental rates in altricial birds. American Naturalist 145: 234 - 260.

Both C, JM Tinbergen und ME Visser 2000: Adaptive density dependance of avian clutch size. Ecology 81: 3391 - 3403.

Boulet M und M Darveau 2000: Depredation of artificial bird nests along roads, rivers, and lakes in a boreal Balsam fir, Abies balsamea, forest. Canadian Field Naturalist 114: 83 - 88.

Browder RG, RC Browder und GC Garman 1995: An inexpensive and automatic multiple-exposure photographic system. Journal of Field Ornithology 66: 37 43.

Brown JL, S Li und N Bhagabati 1999: Long-term trend toward earlier breeding in an American bird: $A$ response to global warming? Proceedings of the National 
Academy of Sciences of the United States of America 96: 5565 - 5569.

Brown KP, H Moller, J Innes und P Jansen 1998: Identifying predators at nests of small birds in a New Zealand forest. Ibis 140: 274 - 279.

Bull EL, RS Holthausen und LR Bright 1992: Comparison of three techniques to monitor marten. Wildlife Society Bulletin 20: 406 - 410.

Bures S 1997: High common vole Microtus arvalis predation on ground-nesting bird eggs and nestlings. Ibis 139: 173 - 174.

Bures S und V Pavel 1997: The effect of nestling condition on risk-taking in Meadow Pipits. Animal Behaviour 54: 1531 - 1534.

Bures S und V Pavel 1998: Is the anti-predative nest defence behaviour of birds primarily determined by the disclosure of the nest site? Biologia e Conservazione della Fauna 102: 97.

Burger LD, LWJ Burger und J Faaborg 1994: Effects of prairie fragmentation on predation on artificial nests. Journal of Wildlife Management 58: 249 - 254.

Burhans DE und FR Thompson III 1998: Effects of time and nest-site characteristics on concealment of songbird nests. Condor 100: 663 - 672.

Burhans DE und FR Thompson III 1999: Habitat patch size and nesting success of yellow-breasted chats. Wilson Bulletin 111: 210 - 215.

Burton JF 1995: Birds and climate change. Helm, London.

Campbell BH und E Lack 1985: A dictonary of birds. BOU, T\&AD Poyser, Calton.

Carthew SM und E Slater 1991: Monitoring animal activity with automated photography. Journal of Wildlife Management 55: 689 - 692.

Charnov EL und JR Krebs 1974: On clutch size and fitness. Ibis 116: 217 - 219.

Collias NE und EC Collias 1984: Nest buliding and bird behaviour. Princeton Aceademic Press, Princeton, New Jersey.

Conell JH 1980: Diversity and the coevolution of competitors, or the ghost of competition past. Oikos 35: 131 - 138.

Conell JH 1983: On the prevalence and relative importance of interspecific competition: evidence from field experiments. American Naturalist 122: 661 - 696.

Conry PJ 1988: High nest predation by brown tree snakes. Condor 90: 478 - 482.

Cresswell W 1997a: Nest predation: The relative effects of nest characteristics, clutch size and parental behaviour. Animal Behaviour 53: 93 - 103.

Cresswell W 1997b: Nest predation rates and nest detectability stages of breeding in blackbirds Turdus merula. Journal of Avian Biology 28: 296 - 302.

Crick HQP, C Dudley, DE Glue und DL Thomson 1997: UK birds are laying eggs earlier. Nature 388: 526 - 526.

Crick HQP und TH Sparks 1999: Climate change related to egg-laying trends. Nature 399: 423 - 424.

Crick HQP, D Wingfield-Gibbons, und Magrath R.D. 1993: Seasonal changes in clutch size in British birds. Journal of Animal Ecology 62: 263 - 273.

Danielson WR, RM De Graaf und TK Fuller 1996: An inexpensive compact automatic camera system for wildlife research. Journal of Field Ornithology 67: 414 - 
421.

Darveau M, L Bélanger, J Huopt, É Mélançon und S Debellefeuille 1997: Forestry practices and the risk of bird nest predation in a boreral coniferous forest. Ecological Applications 7: 572 - 580.

Davison WB und E Bollinger 2000: Predation rates on real and artificial nests of grassland birds. Auk 117: 147 - 153.

Debinski DM und RD Holt 2000: $A$ survey and overview of habitat fragmentation experiments. Conservation Biology 2: 340 - 347.

De Graaf RM 1995: Nest predation rates in managed and reserved extensive northern hardwood forests. Forest Ecology \& Management 79: 227 - 234.

De Graaf RM, TJ Maier und TK Fuller 1999: Predation of small eggs in artificial nests: effects of nest position, edge, and potential predator abundance in extensive forest. Wilson Bulletin 111: 236 - 242.

De Santo TL und MF Willson 2001: Predator abundance and predation of artificial nests in natural and anthropogenic coniferous forest edges in Southeast Alaska. Journal of Field Ornithology 72: 136 - 149.

Deutscher Jagdschutzverband e.V. 1997: Das Schweigen der Sänger. Rabenvögel in der Diskussion.

Dhondt AA und F Adriaensen 1994: Causes and effects of divorce in the blue tit Parus caerulus. Ecology 63: 979 - 987.

Donovan TM, FR Thompson III, J Faaborg und JR Probst 1995: Reproductive success of migratory birds in habitat sources and sinks. Conservation Biology 9: $1380-1395$.

Dow DD 1978: A test of significance for Mayfield's method of calculating nest success. Wilson Bulletin 90: 291 - 295.

Dunn E 1977: Predation by weasels (Mustela nivalis) on breeding tits (Parus spp.) in relation to the density of tits and rodents. Journal of Animal Ecology 46: 633 652.

Dunn PO und DW Winkler 1999: Climate change has affected the breeding date of tree swallows throughout North America. Proceedings of the Royal Society Biological Sciences Series B. 266: 2484 - 2490.

Farnsworth GL, KC Weeks und TR Simons 2000: Validating the assumptions of the Mayfield method. Journal of Field Ornithology 71: 658 - 664.

Filliater TS, R Breitwisch und PM Nealen 1994: Predation on Northern Cardinal nests: Does choice of nest site matter? Condor 96: 761 - 768.

Finch DM 1984: Some factors affecting productivity in Abert's Towhee. Wilson Bulletin 96: $701-705$.

Forbes S und DW Mock 2000: A tale of two strategies: life-history aspects of family strife. Condor 102: 23 - 34.

Forstmeier W, DPJ Kuijper und B Leisler 2001: Polygyny in the dusky warbler, Phylloscopus fuscatus: the importance of female qualities. Animal Behaviour 62: $1097-1108$.

Futuyma DJ 1990: Evolution. Birkhäuser Verlag Basel, Boston, Berlin. 
Gates JE und LW Gysel 1978: Avian nest dispersion and fledging success in fieldforest ecotones. Ecology 59: 871 - 883.

Gatter W 1992: Zugzeiten und Zugmuster im Herbst: Einfluß des Treibhauseffektes auf den Vogelzug? Journal für Ornithologie 133: 427 - 436.

Gärtner K 1982: Zur Ablehnung von Eiern und Jungen des Kuckucks (Cuculus canorus) durch die Wirtsvögel - Beobachtungen und experimentelle Untersuchungen am Sumpfrohrsänger (Acrocephalus palustris). Die Vogelwelt 103:201 - 224.

Ghalambor CK und TE Martin 2000: Parental strategies in two species of nuthatch vary with stage-specific predation risk and reproductive effort. Animal Behaviour 60:263 - 267.

Glutz von Blotzheim UN und KM Bauer 1993: Garrulus glandarius (Linnaeus 1758) Eichelhäher. Handbuch der Vögel Mitteleuropas, Aula Verlag, Wiesbaden $13 /$ III.

Goetz RC 1981: A photographic system for multiple exposures under field conditions. Journal of Wildlife Management 45: 273 - 276.

Göransson G, J Karlsson, SG Nilsson und S Ulfstrand 1975: Predation on bird's nests in relation to antipredator aggression and nest density: an experimental study. Oikos 26: 117 - 120.

Götmark F 1992: Blue eggs do not reduce nest predation in the song thrush, Turdus philomelos. Behavioural Ecology and Sociobiology 30: 245 - 252.

Götmark F, D Blomqvist, OC Johansson und J Bergkvist 1995: Nest site selection: A trade-off between concealment and view of the surroundings? Journal of Avian Biology 26: 305 - 312.

Green RF 1977: Do more birds produce fewer young? A comment on Mayfield's measure of nest success. Wilson Bulletin 89: 173 - 175.

Gustafsson L 1990: Life-history trade-offs and optimal clutch size in relation to age in the collared flycatcher. In: Blondel J, A Gosler, J-D Lebreton, \& R McCleery (Hrsg.): Population Biology of Passerine Birds. Springer-Verlag. S. 235 - 245.

Haartman Lv 1969: The nesting habits of Finnish birds. Communications of the Biological Society Suomi-Fennoscandia. 32: 3 - 187.

Halupka K 1998a: Nest predation in Meadow Pipits Anthus pratensis nesting in natural conditions. Ornis Fennica 75: 139 - 143.

Halupka K 1998b: Nest-site selection and nest predation in meadow pipits. Folia Zoologica 47: 29 - 37.

Hannon SJ und SE Cotterill 1998: Nest predation in aspen woodlots in an agricultural area in Alberta - the enemy from within. Auk 115: 16 - 25.

Hansell M 2000: Bird nests and construction behaviour. Cambridge University Press

Hanski IK und A Laurila 1993: High nest predation rates of the Chaffinch. Ornis Fennica 70: 65 - 70.

Hansson L 1989: Predation in heterogeneous landscapes: how to evaluate total impact? Oikos 54: 117 - 119.

Hartley MJ und ML Hunter 1998: A meta-analysis of forest cover, edge effects, and 
artificial nest predation. Conservation Biology 12: 465 - 469.

Haskell DG 1995: Forest fragmentation and nest predation: are experiments with Japanese Quail eggs misleading? Auk 112: 767 - 770.

Hatchwell BJ, DE Chamberlain und CM Perrins 1996: The reproductive success of Blackbirds Turdus merula in relation to habitat structure and choice of nest site. Ibis 138: 256 - 262.

Heine G, H Jacoby, H Leuzinger und H Stark 1999: Die Vögel des Bodenseegebietes. Ornithologische Jahreshefte für Baden-Württemberg 14/15.

Hensler GL und JD Nichols 1981: The Mayfield method of estimating nesting success: a model, estimators and simulation results. Wilson Bulletin 93: 42 - 53.

Hensley RC und KG Smith 1986: Eastern bluebird responses to nocturnal black rat snake predation. Wilson Bulletin 98: 602 - 603.

Hernandez F, D Rollins, und R Cantu 1997a: An evaluation of Trailmaster camera systems for identifying ground-nest predators. Wildlife Society Bulletin 25: 848 - 853.

Hernandez F, D Rollins und R Cantu 1997b: Evaluating evidence to identify groundnest predators in west Texas. Wildlife Society Bulletin 25: 826 - 831.

Heske EJ, SK Robinson und JD Brawn 1999: Predator activity and predation on songbird nests on forest-field ecotones in east-central Illinios. Landscape Ecology 14: 345 - 354.

Hoi H 1999: Raubdruck und Naturschutz. Internationales Symposium Naturschutz und Verhalten.Göttingen, 4.-6.10.99.

Hoover JP und MC Brittingham 1998: Nest site selection and nesting success of wood thushes. Wilson Bulletin 110: 375 - 383.

Hoover JP, MC Brittingham und LJ Goodrich 1995: Effects of forest patch size on nesting success of wood. Auk 112: 146 - 155.

Howe RW und DavisG.J. 1991: The demographic significance of 'sink' populations. Biological Conservation 57: 239 - 255.

Howlett JS und BJ Stutchbury 1996: Nest concealment and predation in hooded warblers: experimental removal of nest cover. Auk 113: 1 - 9.

Högstedt G 1980: Evolution of clutch size in birds: adaptive variation in relation to territory quality. Science 210: 1148 - 1150.

Huber J 1956: Der Eichelhäher als Nestlingsräuber. Ornithologischer Beobachter 53: $44-44$.

Hughes L 2000: Biological consequences of global warming: is the signal already apparent? Trends in Ecology \& Evolution 15: 56 - 61.

Huhta E 1995: Effects of spatial scale and vegetation cover on predation. Wildlife Biology 1: 73 - 80.

Huhta E, J Jokimaki und P Helle 1998: Predation on artificial nests in a forest dominated landscape - the effects of nest type, patch size and edge structure. Ecography 21: 464 - 471.

Huhta E, T Mappes und J Jokimaki 1996: Predation on artificial ground nests in relation to forest fragmentation, agriculural land and habitat structure. Ecography 
19: $85-91$.

Ilpichino C und B Massa 1989: The birds of Sicily. B.O.U.Check-list No 11. Jacobs GH 1992: Ultraviolett vision in vertebrates. American Zoologist 32: 544 - 554.

Jacobs GH 1993: The distribution and nature of colour vision among the mammals. Biological Review 68: 413 - 471.

Jacobs GH, J Neitz, und JF Deegan 1991: Retinal receptors in rodents maximally sensitive to ultraviolett light. Nature 353: 655 - 656.

Järvinen A 1994: Global warming and the egg size of birds. Ecography 17: 108 - 110.

Järvinen A 1996: Correlation between egg size and clutch size in the Pied Flycatcher Ficedula hypoleuca in cold and warm summers. Ibis 138: 620 - 623.

Johnson DH 1999: The insignificance of statistical significance testing. Journal of Wildlife Management 63: 763 - 772

Julliard R, RH Mccleery J Clobert, und CM Perrins 1997: Phenotypic adjustment of clutch size due to nest. Ecology 78: 394 - 404.

King DI, RM De Graaf, CR Griffin und TJ Maier 1999: Do predation rates on artificial nests accurately reflect predation rates on natural bird nests? Journal of Field Ornithology 70: 257 - 262.

King DI, RM De Graaf, PJ Champlin und TB Champlin 2001: A new method for wireless video monitoring of bird nests. Wildlife Society Bulletin 29: 349 - 353.

Knopf FL und JA Sedgwick 1992: An experimental study of nest-site selection by yellow warblers. Condor 94: 734 - 742.

Kooiker G 1994: Influence of Magpie Pica pica on urban bird populations in the city of Osnabrück. Vogelwelt 115: 39 - 44.

Krebs JR 1971: Territory and breeding density in the Great Tit, Parus major, L. Ecology 52: 2 - 22.

Krebs JR 1990: Food-storing birds: adaptive specialization in brain and behaviour? Philosophical Transactions of the Royal Society of London B Biological Sciences 329: 153 - 160.

Kucera TE und RH Barrett 1993: The Trailmaster camera system for detecting wildlife. Wildlife Society Bulletin 21: 505 - 508.

Kulesza G 1990: An analysis of clutch-size in New World parrerine birds. Ibis 132: $407-422$.

Labitte A 1955: Comparaison entre nos trois fauvettes en Eure-et-Loir. Oiseau 25: $308-311$.

Lack D 1948: The significance of clutch-size. Part III.-Some interspecific comparisons. Ibis 90: 25 - 45.

Lack D 1968: Ecological adaptations of breeding in birds. Methuen, London, England.

Lahti DC 2001: The "edge effect on nest predation" hypothesis after twenty years. Biological Conservation 99: 365 - 374.

Langen TA, DT Bolger und TJ Case 1991: Predation on artificial bird nests in chaparral fragments. Oecologia 86: 395 - 401. 
Latta SC, JMJ Wunderle, E Terranova und M Pagan 1995: An experimental study of nest predation in a subtropical wet forest following hurricane disturbance. Wilson Bulletin 107: 590 - 602.

Leisler B 1981: Die ökologische Einnischung der mitteleuropäischen Rohrsänger (Acrocephalus, Sylviinae).I. Habitattrennung. Die Vogelwarte 31: 35 - 74.

Leisler B und H Winkler 1991: Ergebnisse und Konzepte ökomorphologischer Untersuchungen an Vögeln. Journal für Ornithologie 132: 373 - 425.

Lima SL 1987: Clutch size in birds: a predation perspective? Ecology 68: 1062 1070.

Linden M 1991: Divorce in great tits - chance or choice? An experimental approach. American Naturalist 138: 1039 - 1048.

Liou LW, R Price, MS Boyce und CM Perrins 1993: Fluctuation environments and clutch size evolution in Great Tits. American Naturalist 141: 507 - 516.

Lopez-Iborra G, C Sancho-Urios und F Gonzales-Ortega 2001: Identification of nest predators of Reed Warbler and Great Reed Warbler with time-lapse videos. Programme and abstractbook 3rd EOU Conference, 21-25 August $2001 \mathrm{Ha}-$ ren/Groningen, The Netherlands: 79.

Ludvig É, L Vanicsek, J Török und T Csörgö 1995: Seasonal variation of clutch size in the European blackbird Turdus merula: a new ultimate explanation. Journal of Animal Ecology 64: 85 - 94.

MacArthurR.H. und EO Wilson 1967: The theory of island biogeography. Princeton University Press, Princeton, N.J.

Major RE 1991: Identification of nest predators by photography, dummy eggs, and adhesive tape. Auk 108: 190 - 195.

Major RE, FJ Christie, G Gowing und TJ Ivison 1999: Elevated rates of predation on artificial nests in linear strips of habitat. Journal of Field Ornithology 70: 351 364.

Major RE und CE Kendal 1996: The contribution of artificial nest experiments to understanding avian reproductive success: a review of methods and conclusions. Ibis 138: 298 - 307.

Major RE, GH Pyke, MT Christy, G Gowing und RS Hill 1994: Can nest predation explain the timing of the breeding season and the patterns of nest dispersion of New Holland honeyeaters? Oikos 69: 364 - 372.

Marini MA und C Melo 1998: Predators of Quail eggs, and the evidence of the remains - implication for nest predation studies. Condor 100: 395 - 399.

Marini MA, SK Robinson und EJ Heske 1995: Edge effects on nest predation in the Shawnee National Forest, Southern Illinois. Biological Conservation 74: 203 213.

Marks JS 1986: Nest-site characteristics and reproductive success of long-eared owls on southwestern Idaho. Wilson Bulletin 98: 547 - 560.

Martin TE 1987: Artificial nest experiments: effect of nest appearance and type of predator. Condor 89: 925 - 928.

Martin TE 1988a: Processes organizing open-nesting bird assemblages: competition or nest predation? Evolutionary Ecology 2: 37 - 55. 
Martin TE 1988b: Habitat and area effects on forest bird assemblages: is nest predation an influence? Ecology 69: 74 - 84.

Martin TE 1988c: On the advantage of beeing different: nest predation and the coexistance of bird species. Proceedings of the National Academy of Sciences of the United States of America 1988: 2196 - 2199.

Martin TE 1988d: Nest placement: implications for selected life-history traits, with special reference to clutch size. American Naturalist 132: 900 - 910.

Martin TE 1992: Interaction of nest predation and food limitation in reproductive strategies. Power, D.M.(Ed.).Current Ornithology 9: 163 - 197.

Martin TE 1993a: Nest predation and nest sites. BioScience 43: 523 - 532.

Martin TE 1993b: Nest predation among vegetation layers and habitat types: revising the dogmas. American Naturalist 141: 897 - 913.

Martin TE 1993c: Evolutionary determinants of clutch size in cavity-nesting birds: nest predation or limited breeding opportunities? American Naturalist 142: 937 - 946.

Martin TE 1995: Avian life history evolution in relation to nest sites, nest predation, and food. Ecological Monographs 65: 101 - 127.

Martin TE und J Clobert 1996: Nest predation and avian life-history evolution in Europe versus North America: a possible role of humans. American Naturalist 147: 1028 - 1046.

Martin TE und GR Geupel 1993: Nest-monitoring plots: methods for locating nests and monitoring success. Journal of Field Ornithology 64: 507 - 519.

Martin TE, AP Møller, S Merino und J Clobert 2001: Does clutch size evolve in response to parasites and immunocompetence? Proceedings of the National Academy of Sciences of the United States of America 98: 2071 - 2076.

Martin TE und JJ Roper 1988: Nest predation and nest site selection of a western population of the Hermit Thrush. Condor 90: 51 - 57.

Martin TE, J Scott und C Menge 2001: Nest predation increases with parental activity: separating nest site and parental activity effects. Proceedings of the Royal Society of London - Series B: Biological Sciences 267: 2287 - 2293.

Marzluff JM 1988: Do pinyon jays alter nest placement based on prior experience? Animal Behaviour 36: 1 - 10.

Mason CF 1976: Breeding biology of the Sylvia warblers. Bird Study 23: 213 - 232.

Matessi G und G Bogliani 1999: Effects of nest features surrounding landscape on predation rates of artificial nests. Bird Study 46: 184 - 194.

Matthews A, R Christopher und RE Major 1999: The influence of fragment size and edge on nest predation in urban bushland. Ecography 22: 349 - 356.

Maxson SJ und LW Oring 1978: Mice as a source of nest loss among ground nesting birds. Auk 95: 582 - 584.

Mayfield H 1961: Nesting success calculated from exposure. Wilson Bulletin 73: 255 $-261$.

Mayfield HF 1975: Suggestions for calculating nest success. Wilson Bulletin 87: 456 466. 
Mäck U und M-E Jürgens 1999: Aaskrähe, Elster und Eichelhäher in Deutschland. Bundesamt für Naturschutz, Bonn.

Mclean IG und G Rhodes 1991: Enemy recognition and response in birds. Power, D.M.(Ed.).Current Ornithology, 8: 173 - 211. Plenum Press, New York.

McQuillen HL und LW Brewer 2000: Methodological considerations for monitoring wild bird nests using video technilogy. Journal of Field Ornithology 71: 167 172.

Meilvang D, A Moksnes und E Roskaft 1997: Nest predation, nesting characteristics and nest defence behaviour of fieldfares and redwings. Journal of Avian Biology 28: 331 - 337.

Metzler G 2001: Vergleichende Untersuchungen der Brutbiologie von Teich- und Drosselrohrsänger (Acrocephalus scirpaceus Herm., 1804, Acrocephalus arundinaceaus L., 1758) in einem Weihergebiet. Diplomarbeit Universität Göttingen.

Miller CK und RL Knight 1993: Does predator assemblage effect reproductive success in songbirds? Condor 95: 712 - 715.

Miller HW und DH Johnson 1978: Interpreting the results of nesting studies. Journal of Wildlife Management 42: 471 - 476.

Moreau RE 1944: Clutch size: A comperative study, with special reference to african birds. Ibis 86: 286 - 347.

Moreno J und JJ Sanz 1994: Experimentally induced clutch size enlargements affect reproductive success in pied flycatchers Ficedula hypoleuca. Journal für Ornithologie 135: 12.

Morris DW 1991: On the evolutionary stability of dispersal to sink habitats. The American Naturalist 137: 907 - 911.

Morton ML, KW Sockman und LE Peterson 1993: Nest predation in the mountain white-crowned sparrow. Condor 95: 72 - 82.

Mousley $\mathrm{H}$ 1917: A study of subsequent nestings after the lost of the first. Auk 34: $381-393$.

Møller AP 1982: Clutch size in relation to nest size in the swallow Hirundo rustica. Ibis 124: 339 - 343.

Møller AP 1988: Nest predation and nest site choice in passerine birds in habitat patches of different size: a study of magpies and blackbirds. Oikos 53: 215 - 221.

Møller AP 1989: Nest site selection across field forest ecotones: the effect of nest predation. Oikos 56: 240 - 246.

Møller AP 1990: Nest predation selects for small nest size in the blackbird. Oikos 57: $237-240$.

Møller AP 1994: Sexual selection and the barm swallow. Oxford University Press, Oxford.

Murcia C 1995: Edge effects in fragmented forests: implications for conservation. TREE 10: 58 - 62.

Murphy EC 1978: Seasonal variation in reproductive output of House Sparrows: The determination of clutch size. Ecology 59: 1189 - 1199. 
Murphy EC und E Haukioja 1993: Clutch size in nidicolous birds. Current Ornithology 4: $141-180$.

Nager RG 1994: Plasticity of Great Tit's laying date: Effects of local temperature and food avaiability. Journal für Ornithologie 135: 345.

Nager RG und AJ van Noordwijk 1995: Proximate and ultimate aspects of phenotypic plasticity in timing of great tit breeding in a heterogeneous environmentq. American Naturalist 146: 454 - 474.

Nice MM 1957: Nesting success in altricial birds. Auk 74: 305 - 321.

Nilsson SG 1984: The evolution of nest-site selection among hole-nesting birds: the importance of nest predation and competition. Ornis Scandinavica 15: 167 175.

Nockemann L 1998: Prädationsdruck auf ufernahe Boden- und Schwimmnester in Schilfgürteln Masurischer Seen, Nordostpolen. Diplomarbeit Universität Göttingen.

Nour N, E Matthysen und AA Dhondt 1993: ARTIFICIAL NEST PREDATION AND HABITAT. Ecography 16: $111-116$.

Onnebrink H und E Curio 1991: Brood defense and age of young: a test of the vulnerability hypothesis. Behavioral Ecology \& Sociobiology 29: 61 - 68.

Opdam P 1991: Metapopulation theory and habitat fragmentation: a review of holarctic breeding bird studies. Landscape Ecology 6: 93 - 106.

Parker $\mathrm{H}$ 1984: Effect of corvid removal on reproduction of willow ptarmigan. Journal of Wildlife Management 48: 1197 - 1205.

Paton PW 1994: The effect of edge on avian nest success: How strong is the evidance? Conservation Biology 8: 17 - 26.

Pätzold R 1983: Die Feldlerche. A. Ziemsen Verlag, Wittenberg Lutherstadt.

Pennington MG 1992: Predation of birds' eggs and chicks by herbivorous mammals. Scottish Birds 16: 285 - 285.

Perrins CM 1970: The timing of birds' breeding seasons. Ibis 112: 242 - 255.

Picman J. 1987: An inexpensive camera setup for the study of egg predation at artificial nests. Journal of Field Ornithology 58: 372 - 382.

Picman J und LM Schriml 1994: A camera study of temporal patterns of nest predation in different habitats. Wilson Bulletin 106: 456 - 465.

Pietz PJ und DA Gransfors 1998: A miniature camera system for studies of grassland passerine nests. Biological Information \& Technology Notes

Pietz PJ und DA Gransfors 1998: A potential tool for documenting activities at cryptic grassland passerine nests. Ostrich 69: 302 - 302.

Pietz PJ und DA Gransfors 2000: Identifying predators and fates of grassland passerine nests using miniature video cameras. Journal of Wildlife Management 64: $71-87$.

Pulido F 2000: Evolutionary quantitative genetics of migratory restlessness in the blackcap (Sylvia atricapilla).Tectum Verlag Marburg.

Pulliam HR und BJ Danielson 1997: Sources, sinks and habitat selection: a landscape perspective on populations dynamics. American Naturalist 137: 50 - 66 . 
Reyer HU 1988: Ökolgie und Evolution von kooperativer Jungenaufzucht bei Vögeln. Verhandlungen der Deutschen Zoologischen Gesellschaft 81: 169 - 182.

Ricklefs RE 1969: An analysis of nesting mortality in birds. Smithonian Contributions of Zoology 9: 1 - 48.

Ricklefs RE 2000: Lack, Skutch, and Moreau: The early development of life-history thinking. Condor 102: 3 - 8.

Robbins CS, JR Sauer und R Greenberg 1989: Population declines in North American birds that migrate to the Neotropics. Proceedings of the National Academy of Science US 86: 7658 - 7662.

Robinet O, JL Craig und L Chardonnet 1998: Impact of rat species in Ouvea and Lifou (Loyalty Islands) and their consequences for conserving the endangered Ouvea parakeet. Biological Conservation 86: 222 - 232.

Rotenberry JT und JA Wiens 1980: Temporal variation in habitat structure and shrubsteppe bird dynamics. Oecologia 47: 1 - 9.

Rothhaupt G 1998: Populationsgefährdungsanalyse beim Raubwürger (Lanius excubitor L.). Cuvillier Verlag, Göttingen.

Roughgarden J 1983: Competition and theory in community ecology. American Naturalist 122: 583 - 601 .

Santos T und JL Tallería 1992: Edge effect on nest predation in Mediterranean fragmented forests. Biological Conservation 60: 1 - 5 .

Savidge JA 1987: Extinction of an island forest avifauna by an introduced snake. Ecology 68: 660 - 668.

Scebba S 1993: Gli uccelli della Campania-. Monografia N1, Edizioni Esselibri, Napoli.

Schaefer T 2001: Folgen der Klimaerwärmung: Brutbiologie der Mönchsgrasmücke heute und vor 20 Jahren. In: G Zotz und C Körner (Hrsg.): Funktionelle Bedeutung von Biodiversität. Parey, Berlin. S. 39.

Schaefer T 2002: Nest predation and renesting in the blackcap. Vogelkundliche Berichte Niedesachsen 34: im Druck.

Schaefer T und G Heine 2002: Test of an inexpensive camera set for nest and animal activity observations. Vogelwarte 42: im Druck.

Schaefer VH 1976: Geographic variation in the placement and structure of Oriole nests. Condor 78: 443 - 448.

Schmidt KA und CJ Whelan 1999: Nest placement and mortality: is nest predation a random event in space and time? Condor 101: 916 - 920.

Schoener TW 1982: The controversy over interspecific competition. American Scientist 70: 556 - 595.

Schoener TW 1983: Field experiments on interspecific competition. American Naturalist 122: 240 - 285.

Schulze-Hagen K, B Leisler und H Winkler 1996: Breeding success and reproductive strategies of two Acrocephalus warblers. Journal für Ornithologie 137: 181 192.

Scott DM, RE Lemo, und JA Darley 1987: Relaying intervall after nest failure in Gray 
Catbirds and Northern Cardinals. Wilson Bulletin 99: 708 - 712.

Sell K 1998: Dauerbeobachtung von Singvogelnestern mit Videokameras - eine Pilotstudie. Examensarbeit, Universität Konstanz.

Shettleworth SJ 1990: Spacial memory in food storing birds. Philosophical Transactions of the Royal Society of London B Biological Sciences 329: 143 - 152.

Sieving KE 1992: Nest predation and differential insular extinction among selected forest birds of central Panama. Ecology 73: 2310 - 2328.

Sih A, G Englund und D Wooster 1998: Emergent impact of multiple predators on prey. Trends in Ecology \& Evolution 13: 350 - 355.

Skutch AF 1949: Do tropical birds rear as many young as they can nourish? lbis 91: $430-455$.

Slagsvold T 1982: Clutch size variation in passerine birds: the nest predation hypothesis. Oecologia 54: 159 - 169.

Slagsvold T 1984: Clutch size variation of birds in relation to nest predation: on the cost of reproduction. Journal of Animal Ecology 53: 945 - 954.

Slagsvold T 1989: Experiments on clutch size and nest size in passerine birds. Oecologia 80: 297 - 302.

Slagsvold T und JT Lifjeld 1986: Mate retention and male polyterritoriality in the pied fycatcher Ficedula hypoleuca. Behavioral Ecology \& Sociobiology 19: 25 - 30.

Sockman KW 1997: Variation of life-history traits and nest-site selection affects risk of nest predation in the California Gnatcatcher. Auk 114: 324 - 332.

Sonerud GA und PE Fjeld 1987: Long-term memory in egg predators an experiment with the hooded craw. Ornis Scandinavica 18: 323 - 324.

Sovada MA, MC Zicus, RJ Greenwood, DP Rave, WE Newton, RO Woodward und JA Beiser 2000: Relationships of habitat patch size to predator community and survival of duck nests. Journal of Wildlife Management 64: 820 - 831.

Söderstrom B 1999: Artificial nest predation rates in tropical and temperate forests: a review of the effects of edge and nest site. Ecography 22: 455 - 463.

Stamps JA 1988: Conspecific atrraction and aggregation in territorial species. American Naturalist 131: 329 - 347.

Stein H 1974: Ein Beitrag zur Brutbiologie von Singdrossel, Turdus philomelos, Amsel, Turdus merule, und Mönchsgrasmücke, Sylvia atricapilla mit besonderer Berücksichtigung der Brutverluste. Beiträge zur Vogelkunde 20: 467 - 477.

Suarez F, M Yanes, J Herranz, und J Manrique 1993: Nature reserves and the conservation of Iberian shrubsteppe passerines: the paradox of nest predation. Biological Conservation 64: 77 - 81.

Thomas CD und JJ Lennon 1999: Birds extend their ranges northwards. Nature 399:213.

Thomas DW, J Blondel, P Perret, MM Lambrechts und JR Speakman 2001: Energetic and fitness costs of mismatching resource supply and demand in seasonally breeding birds. Science 291: 2598 - 2600.

Thompson FRI, W Dijak und DE Burhans 1999: Video identification of predators at songbird nests in old fields. Auk 116: 259 - 264. 
Trine CL 1998: Wood thrush population sinks and implications for the scale of regional. Conservation Biology 12: 576 - 585.

van Noordwijk AJ 1987: 11 Quantitative ecological genetics of Great Tits. In: Cooke, F.\& Buckley, P.A.: Avian genetics.A population und ecological approach.Academic Press, London. S. 363 - 379.

van Noordwijk AJ, JH van Balen und W Scharloo 1980: Heritability of ecological important traits in the Great Tit. Ardea 68: 193 - 203.

Visser ME, AJ van Noordwijk, JM Tinbergen und CM Lessells 2002: Warmer springs lead to mistmed reproduction in great tits (Parus major). Proceedings of the Royal Society of London - Series B: Biological Sciences 265: 1867 - 1870.

Vogel B 1998: Habitatqualität oder Landschaftsdynamik - Was bestimmt das Überleben der Heidelerche (Lullula arborea)? Cuvillier Verlag Göttingen.

Vogrin M und N Vogrin 1998: Bird communities of intensively cultivated fields in North-eastern Slovenia. Acta Ornithologica 33: 173 - 179.

Wallander $\mathrm{J}$ und M Andersson 2002: Clutch size limitation in waders: experimental test in redshank Tringa totanus. Oecologia 130: 391 - 395.

Walsberg GE 1985: Physiological consequences of microhabitat selection. In: Cody, M.L.(Ed.): Habitat selection in birds. Orlando Acad.press. S. 389-413,

Weidinger K 2001: How well do predation rates on artificial nests estimate predation on natuzral passerine nests? lbis 143: 632 - 641.

Widmer M 1999: Die Mönchsgrasmücke-Sylvia atricapilla. Heine, G., H.Jacoby, H.Leuzinger \& H.Stark: Die Vögel des Bodenseegebietes.Ornithologische Jahreshefte Baden-Wüttemberg 14/15.

Wiens JA 1977: On competition and variable environments. American Scientist 65: $590-597$.

Wiens JA und JT Rottenberry 1979: Diet niche relationships among North America, grassland and shrubsteppe birds. Oecologia 42: 253 - 292.

Wiggins DA, T Pärt und L Gustafsson 1994: Correlates of clutch desertion by female Collared Flycatchers Ficedula albicollis. Journal of Avian Biology 25: 93 - 97.

Wilson RR und RJ Cooper 1998: Acadian Flycatcher nest placement: Does placement influence reproductive success? Condor 100: 673 - 679.

Wilson WH, D Kipervaser und SA Lilley 1998: Spring arrival dates of Maine migratory breeding birds: 1994-1997 vs. 1899-1911. Northeastern Naturalist 7.

Winkel W 1997: Long term trends in reproductive traits of tits (Parus major, $P$. caeruleus) and Pied Flycatchers Ficedula hypoleuca. Journal of Avian Biology 28: 187 - 190.

Winkler DW 1987: A general model for parental care. American Naturalist 130: 526 543.

Yahner $\mathrm{RH}$ und BL Cyper 1987: Effects of nest location on depredation of artificial arboreal nests. Journal of Wildlife Management 51: 178 - 181.

Yahner RH und CG Mahan 1996: Depredation of artificial ground nests in a managed, forested landscape. Conservation Biology 10: 285 - 288.

Yahner RH, TE Morell,und JS Rachael 1989: Effects of edge contrast on depredation 
of artificial avian nests. Journal of Wildlife Management 53: 1135 - 1138.

Yahner $\mathrm{RH}$ und $\mathrm{NH}$ Piergallini 1998: Effects of microsite selection on predation of artificial ground nests. Wilson Bulletin 110: 439 - 442.

Yahner RH und A Scott 1988: Effects of forest fragmentation on depredation of artificial nests. Journal of Wildlife Management 52: 158 - 161.

Yahner $\mathrm{RH}$ und AL Wright 1985: Depredation on artificial ground nests: effects of edge and plot age. Journal of Wildlife Management 49: 508 - 513.

Yanes M, J Herranz und F Suarez 1996: Nest microhabitat selection in larks from European semi-arid shrub-steppe: the role of sunlight and predation. Journal of Arid Environments 32: 469 - 478.

Yanes M und F Suarez 1995: Nest predation patterns in ground-nesting passerines in the Iberian Peninsula. Ecography 18: 423 - 42 


\section{Anhang I: Tabellenverzeichnis}

Tab. 1: Anzahl der in den Untersuchungsflächen zwischen 1981 und 2000 gefundenen Nester.

Tab. 2: Kennwerte der multiplen Regression zum Test der Abhängigkeit der täglichen Überlebenswahrscheinlichkeit von Jahr und Fundort $(n=105)$.

Tab. 3: Anzahl von Nestern (NN), Nestern mit Daten zum Bruterfolg (BE) und erfolgreicher Nester (EN); Summe (SB) und Mittelwert (MB) der Beobachtungstage, tägliche Überlebenswahrscheinlichkeit (tÜ) in den sieben Untersuchungsflächen der Nestersuche 1981-2000.

Tab. 4: Z-Werte (fett), Differenzen und summierte Standardabweichung (kursiv) der Berechnungen nach Hensler \& Nichols (1981) für Unterschiede der täglichen Überlebenswahrscheinlichkeit zwischen den Untersuchungsflächen. Z-Werte über 1,65 zeigen Unterschiede auf einem Signifikanzniveau von $0,05 \ldots . . .44$

Tab. 5: Fläche (F), Randlänge (R) und Rand/Fläche-Index (R/F), mittlere (EW) und minimale (MW) Entfernung zum nächsten Wald und Waldanteile auf $1 \mathrm{~km}^{2}$ (W 1) und $4 \mathrm{~km}^{2}$ (W 4) Umgebung der sieben Untersuchungsflächen.

Tab. 6: Verlustursachen von Nestern der Mönchsgrasmücke nach den

Videoüberwachungen.

Tab. 7: Prädatoren und deren jeweilige Anteile an Prädationen, Verlusten und insgesamtem Ergebnis aller Nester.

Tab. 8: Anzahl der Besuche der Eichelhäher am Nest und der Tage, über die sich die Besuche erstreckten.

Tab. 9: Verlustursachen und Spuren am Nest $(n=80)$.

Tab. 10: Anteile einzelner Prädatoren an Nestern mit (alle Ereignisse) und ohne (einfach) Doppelbeobachtungen an einem Nest und der Erstprädatoren an einem Nest (Anzahl Beobachtungen). 50

Tab. 11: Saisonalität der Prädatoren an Fotofallen mit allen Beobachtungen ( $a, p<$ $0,025)$ und unter Ausschluss von Mehrfachbeobachtungen $(e, p<0,005)$ am gleichen Nest.

Tab. 12: Datum und Uhrzeit der Besuche von Waldmäusen am Nest 84/2000.53

Tab. 13: Übereinstimmungen von Prädatoren an aktiven Nestern (V) und Fotofallen (F 1-5, E: Eichelhäher. S: Steinmarder, F: Fuchs, H: Haselmaus, N: Nager; K: Kohlmeise). 
Tab. 14: Kennwerte der Tests zum Vergleich erfolgreicher und nicht erfolgreicher Nester hinsichtlich der gemessenen Parameter. 60

Tab. 15: Kennwerte der Tests zum Vergleich extremer und mittlerer Standorte mit den verbleibenden Nestern.

Tab. 16: Erfolgreiche (e) und nicht erfolgreiche (n) Fotofallennester auf Mallorca und in SW-Deutschland ( $\left.{ }^{2}=12,5, \mathrm{p}<0,0004\right)$. 65

Tab. 17: Erfolgreiche (e) und nicht erfolgreiche (n) Kunstnester auf Mallorca und in SW-Deutschland im Juni $\left({ }^{2}=17,6, p<0,0001\right)$ und Juli $\left({ }^{2}=11,7, p<0,0006\right)$. 65

Tab. 18: Anzahl und Auftretenswahrscheinlichkeit verschiedener Gelegegrößenklassen der Nestfunde ( $n=1424)$ 69

Tab. 19: Mittelwerte und Standardfehler der Gelegegröße in den

Untersuchungsflächen des Nestfundes. Die Flächen unterscheiden sich hinsichtlich der Gelegegröße (ANOVA, $p<0,001$ ) 69

Tab. 20: Unterschiede in der Gelegegröße zwischen den Untersuchungsflächen unter Berücksichtigung der Kovariaten "Legebeginn" und "Jahr" (ANCOVA, $p<$ $0,001)$. 69

Tab. 21: Kennwerte der logistischen Regression zwischen Gelegegröße und täglicher Überlebenswahrscheinlichkeit (tÜ) in einem Waldstück, dem Fundjahr und dem Legebeginn $(n=1492)$. 71

Tab. 22: Kennwerte der Multiplen Regression für die Abhängigkeit der Hauptlegephase vom Jahr und der täglichen Überlebenswahrscheinlichkeit (tÜ) und Werte der Power-Analyse (KSW: kleinster signifikanter Wert, $n=20$ ). 70 Tab. 23: Kennwerte der Multiplen Regression für die Abhängigkeit des frühesten Legebeginnes vom Jahr und von der täglichen Überlebenswahrscheilichkeit (tÜ) und Werte der Power-Analyse (s.o.). 70

Tab. 24: Kennwerte der logistischen Regression zwischen Kalendereffekt, natürliche Überlebenswahrscheinlichkeit und der Gelegegröße als abhängiger Variable $(n=$ 20). 72

Tab. 25: Kennwerte der logistischen Regression zwischen Kalendereffekt, Überlebenswahrscheinlichkeit nach Entnahme von Versuchsvögeln und der Gelegegröße als abhängiger Variable $(n=20)$ 72

Tab. 26: Tägliche Überlebenswahrscheinlichkeit (tÜ) der einzelnen

Gelegegrößenklassen. 


\section{Anhang II: Abbildungsverzeichnis}

Abb. 1: Mögliche Adaptionen an Nestprädation.

Abb. 2: Schematische Darstellung der Lebensformen der Mönchsgrasmücke. Z: ausschließlich Zugvögel, T: Teilzieher, S: Standvögel, dicke Pfeile: Haupt-, dünne Pfeile: Nebenzug-richtungen, gestrichelt: äußere Grenze der Brutverbreitung, schraffiert: Überwinterungsgebiete (aus BERTHOLD 2000 nach BERTHOLD 1999).

Abb. 3: Der westliche Bodenseeraum mit der Lage der Untersuchungsgebiete bei Radolfzell (Kasten).

Abb. 4: Bruthabitate der Mönchsgrasmücke in SW-D. oben: Hecken in einem Tal, unten: Waldrand, rechts: Lichtung.

Abb. 5: Die Untersuchungsflächen in der Umgebung der Dörfer Wahlwies, Stahringen, Liggeringen, Güttingen und Möggingen (S: Schlosshalde, B: Böhlerberg, N: Nusshalde, D: Durchenberg, M: Schloss Möggingen, Z: Ziegelhofweg, H: Hirschbrunnen, K: Kuhbühl, W: Wolfsgrund).

Abb. 6: Lage der Untersuchungsflächen in der Bucht von Alcudia im Nordosten Mallorcas.

Abb. 7: Bruthabitate der Mönchsgrasmücke auf Mallorca. 20

Abb. 8: Schema der verwendeten Videosysteme (links: HR, rechts: LB). 22

Abb. 9: Transportkiste mit Rekorder, Monitor und Akku. 22

Abb. 10: HR-Kamera an einem Nest. 24

Abb. 11: LB-Kamera an einem Nest. 24

Abb. 12: Schema der Fotofalle im Feld. $\quad 27$

Abb. 13: Fotofalle an einem Neststandort, Sicht von hinten auf Wetterschutz. 28

Abb. 14: Schema der aufgenommenen Neststandortparameter. 29

Abb. 15: Kunstnest an einem Neststandort auf Mallorca. 30

Abb. 16: Die Untersuchungsflächen für die Nestersuche an der Vogelwarte im Umkreis von Radolfzell. E1: Espasingen I, E2: Espasingen II, Er: Erdbeerwäldchen, S: Schloss Möggingen, B: Böhringen, M: Mooswald, G: 
Großer Moos.

Abb. 17: Blick in zwei der Untersuchungsflächen, oben: Erdbeerwäldchen, unten:

Böhringen.

Abb. 18: Beispiel der Skizze eines Waldstücks mit den am betreffenden Suchtag

(31.05.2000) gefundenen Nestern.

Abb. 19: Schema der für die untersuchten Waldstücke erfassten Parameter. W:

Waldstück, N: Nachbarwald, durchgezogene Pfeile: Entf. in vier Richtungen, gepunkteter Pfeil: kürzeste Entf. zu einem Waldrand, gestricheltes Quadrat: Fläche mit 1 km und gepunktetes Quadrat: mit 2 km Kantenlänge. $\quad 36$

Abb. 20: Fundtag der Nester nach Legebeginn $(n=2194)$.

Abb. 21: Tag des Verlustes nach Legebeginn $(n=587)$.

Abb. 22: Saisonale Abhängigkeit der täglichen Überlebenswahrscheinlichkeit $\left(\mathrm{r}^{2}=\right.$ $0,99, \mathrm{n}=8, \mathrm{p}<0,0001)$.

Abb. 23: Anzahl gefundener Nester in einer Monatshälfte und tägliche Überlebenswahrscheinlichkeit (n.s.).

Abb. 24: Zusammenhang zwischen der täglichen Überlebenswahrscheinlichkeit und der Flächengröße $\left(r^{2}=0,79, n=7, p=0,0078\right)$.

Abb. 25: Zusammenhang zwischen der täglichen Überlebenswahrscheinlickeit und dem Waldanteil auf einer umgebenden Fläche von $1 \mathrm{~km}$ Kantenlänge $\left(\mathrm{r}^{2}=0,51\right.$, $\mathrm{n}=7, \mathrm{p}=0,074$, n.s.). 45

Abb. 26: Eichelhäher entnimmt ein Ei aus einem Nest (4/1999).

Abb. 27: Uhrzeit der Prädationsereignisse $(n=44)$.

Abb. 28: Aufenthaltsdauer der Prädatoren am Nest (t-Test, $\mathrm{p}<0,0001, \mathrm{n}=30) .48$

Abb. 29: Überlebensdauer ab Legebeginn in Tagen von Video- und Fotofallennestern (t-Test, $\mathrm{p}<0,0026, \mathrm{n}=116$ ).

Abb. 30: Eichelhäher und Haselmaus an einer Fotofalle.

Abb. 31: Uhrzeit der Prädationen an Fotofallen $(n=94)$.

Abb. 32: Waldmaus am Nest 84/2000.

Abb. 33: Dauer bis zum letzten Besuch eines Altvogels nach Prädation (Weibchen: $n$ 
= 8, Männchen: $\mathrm{n}=6$, n.s.).

Abb. 34: Anzahl der Besuche nach Prädation (Weibchen $n=8$, Männchen $n=6$, n.s.).

Abb. 35: Nistpflanzen der Mönchsgrasmücke in der Umgebung von Radolfzell aus Daten der Freilandstudie $(n=152)$.

Abb. 36: „Andere“ (vgl. Abb. 35) Nistpflanzen der Mönchsgrasmücke $(n=23) .56$

Abb. 37: Höhe der Nistpflanze $(n=121)$ und Nesthöhe $(n=141)$, Höhe zur verbesserten Darstellung logarithmiert.

Abb. 38: \% der Nesthöhe von der Nistpflanzenhöhe $(n=121)$ und \% der Entfernung zum Rand vom Durchmesser der Pflanze $(n=54)$.

Abb. 39: Breite der Nistpflanzen $(n=54)$ und Entfernung des Nestmittelpunktes zum Rand der Pflanze $(n=54)$.

Abb. 40: Sichtbarkeit der Nester von oben $(n=138)$, von unten $(n=135)$ und von der Seite (Mittelwert aus den vier gemessenen Richtungen, $n=138$ ).

Abb. 41: Mittelwerte der Sichtbarkeit aus allen Richtungen, von oben und der Seite sowie Minimal- und Maximalwerte aus allen Richtungen.

Abb. 42: Entfernung der Nester zum Wandrand in Metern $(n=137)$.

Abb. 43: Zunahme der Nesthöhe mit der Saison. Offene Kreise: Minimal-, Dreiecke: Maximalwerte, geschl. Kreise: Mittelwert mit Standardabweichung.

Abb. 44: Änderung der Zusammensetzung der Nistpflanzen in den Monaten Mai, Juni und Juli $\left(n=151, \chi^{2}=22.8, p<0,03\right)$.

Abb. 45: Box Plots der Nesthöhe erfolgreicher Nester und der Nester, die von Rabenvögeln oder Säugern ausgeräumt wurden (ANOVA, $p<0,05, n=91$ ).

Abb. 46: Mittelwert und Standardabweichung der Randnähe erfolgreicher Nester und der Nester, die von Rabenvögeln oder Säugern geleert wurden (ANOVA, $p<$ $0,05, n=30)$.

Abb. 47: Nistpflanzen der Mönchsgrasmücke auf Mallorca $(n=64)$.

Abb. 48: Nest der Mönchsgrasmücke in einem Mastixstrauch auf Mallorca (aus Videoaufzeichnungen). 
Abb. 49: Höhe von Nestern der Mönchsgrasmücke auf Mallorca $(n=64)$ und in SW-

$D(n=96, t-T e s t, p<0,001)$.

Abb. 50: Höhe von Nistpflanzen der Mönchsgrasmücke auf Mallorca $(n=64)$ und in SW-D $(n=96, t-T e s t, p<0,005)$.

Abb. 51: Breite der Nistpflanze in Nesthöhe auf Mallorca $(n=64)$ und in SW-D $(n=$ 52, t-Test, $\mathrm{p}<0,02)$.

Abb. 52: Relative Höhe von Nestern der Mönchsgrasmücke auf Mallorca $(n=64)$ und in SW-D $(n=52$, t-Test, $p<0,001)$.

Abb. 53: Relative Nestentfernung zum Rand der Nistpflanze auf Mallorca $(n=63)$ und in SW-D $(n=51, t-T e s t, p<0,001)$.

Abb. 54: Laterale Sichtbarkeit von Nestern auf Mallorca $(n=59)$ und in SW-D $(n=$ 91, t-Test, $\mathrm{p}<0,001)$.

Abb. 55: Nachlegedauer in den Volieren $(n=122)$.

Abb. 56: Saisonale Abhängigkeit der Nachlegedauer in den Volieren $\left(r^{2}=0,034, p<\right.$ $0,03, n=122$ ).

Abb. 57: Nachlegedauer in Abhängigkeit der Brutversuche eines Paares (ANOVA, $p$ $<0,023, \mathrm{n}=122)$.

Abb. 58: Früheste (schwarze Kreise) und späteste Legebeginne (Dreiecke) eines Jahres sowie Median des Legebeginns (weiße Kreise) der Nestfunde seit 1981.

Abb. 59: Anzahl der Legebeginne pro Tag $(n=2201)$ aus den Nestfunden. 68 Abb. 60: Abhängigkeit der Gelegegröße von der Saison nach Mittelwerten in einer Monatshälfte aus Daten der Freilandstudie.

Abb. 61: Abhängigkeit der Gelegegröße von der Saison nach Mittelwerten in einer Monatshälfte aus den Nestfunden.

Abb. 62: Zusammenhang zwischen frühestem Legebeginn und

Überlebenswahrscheinlichkeit im Vorjahr $\left(r^{2}=0,19\right.$, n.s.).

Abb. 63: Zusammenhang zwischen der Hauptlegephase und der

Überlebenswahrscheinlichkeit im Vorjahr $\left(r^{2}=0,29, p<0,024\right)$.

Abb. 64: Zusammenhang zwischen der Gelegegröße und der Prädationsrate im 
Vorjahr (schwarze Kreise: Natürliche Prädation, $r^{2}=0,16, p<0,0823$; offene Kreise nach Entnahme von Versuchsvögeln, $r^{2}=0,38, p<0,0046$.

Abb. 65: Anzahl festgestellter Prädatoren in Untersuchungsgebieten mit unterschiedlichen Prädationsraten (berechnet nach Angaben von MILLER \& KNIGHT 1993), ANOVA, $p<0,0134, n=28$ ).

Abb. 66: Nesterstandorte der Mönchsgrasmücke auf Mallorca und in Südwestdeutschland (aus den Mittelwerten, schematisch). 